\title{
EFFICIENT ESTIMATION IN A REGRESSION MODEL WITH MISSING RESPONSES
}

\author{
A Dissertation \\ by \\ SCOTT DANIEL CRAWFORD
}

\begin{abstract}
Submitted to the Office of Graduate Studies of Texas A\&M University in partial fulfillment of the requirements for the degree of DOCTOR OF PHILOSOPHY
\end{abstract}

August 2012

Major Subject: Statistics 


\title{
EFFICIENT ESTIMATION IN A REGRESSION MODEL WITH MISSING RESPONSES
}

\author{
A Dissertation \\ by \\ SCOTT DANIEL CRAWFORD
}

\begin{abstract}
Submitted to the Office of Graduate Studies of Texas A\&M University

in partial fulfillment of the requirements for the degree of

DOCTOR OF PHILOSOPHY
\end{abstract}

Approved by:

Chair of Committee, Ursula U. Mueller-Harknett

Committee Members, Jeff Hart

Soumendra N. Lahiri

Joel Zinn

Head of Department, Simon J. Sheather

August 2012

Major Subject: Statistics 


\author{
ABSTRACT \\ Efficient Estimation in a Regression Model \\ with Missing Responses. (August 2012) \\ Scott Daniel Crawford, B.S., Southern Utah University; \\ M.S., Brigham Young University; \\ Chair of Advisory Committee: Dr. Ursula U. Mueller-Harknett
}

This article examines methods to efficiently estimate the mean response in a linear model with an unknown error distribution under the assumption that the responses are missing at random. We show how the asymptotic variance is affected by the estimator of the regression parameter and by the imputation method. To estimate the regression parameter the Ordinary Least Squares method is efficient only if the error distribution happens to be normal. If the errors are not normal, then we propose a One Step Improvement estimator or a Maximum Empirical Likelihood estimator to estimate the parameter efficiently.

In order to investigate the impact that imputation has on estimation of the mean response, we compare the Listwise Deletion method and the Propensity Score method (which do not use imputation at all), and two imputation methods. We show that Listwise Deletion and the Propensity Score method are inefficient. Partial Imputation, where only the missing responses are imputed, is compared to Full Imputation, where both missing and non-missing responses are imputed. Our results show that in general Full Imputation is better than Partial Imputation. However, when the regression parameter is estimated very poorly, then Partial Imputation will outperform Full Imputation. The efficient estimator for the mean response is the Full Imputation estimator that uses an efficient estimator of the parameter. 


\section{ACKNOWLEDGMENTS}

A great debt of gratitude goes to my advisor, Dr. Ursula U. Mueller-Harknett, for her help, support, enthusiasm, and interest in my work. She gave me time every week to teach, expound ideas, and broaden my understanding of statistics. Without her help I would never have made sense of that Hajek Le-Cam theory. She is an example of what a researcher should be, and one of a few experts in the field of efficiency and missing data models. Great things can be expected from her and her students in the future.

Thanks to Anton Schick for his amazing insights and expertise with the trickiest forms of the convolution theorem. Also thanks to Wolfgang Wefelmeyer for several professional discussions. Thanks to Paul Martin, to Julie Carroll, to Ellen Toby, and especially Micheal Longnecker for help and encouragement when I needed it most. The entire staff, faculty, and student body of Texas A\&M are known for their quality. The Texas A\&M University Brazos HPC cluster made the simulations possible.

Thanks to my dear wife, Teasha, who has waited patiently for me to finish this journey and supported me with love and comfort every step of the way. Without Teasha none of my accomplishments would matter, but with her I feel as though I am the most blessed man alive. A thanks to God that sent me such a woman and strengthened me through trials and triumphs alike. I am proud of this work because it is the sum of divine help, my efforts, outstanding academic advising, and the support of friends and family. 
TABLE OF CONTENTS

CHAPTER

Page

I

INTRODUCTION . . . . . . . . . . . . . . . 1

II $\square$ PARAMETER ESTIMATION IN LINEAR REGRESSION . . 4

A. The model . . . . . . . . . . . . . . . . 4

B. Estimating the parameter . . . . . . . . . . . . 5

1. Ordinary least squares estimator . . . . . . . . . 7

a. OLS with double exponential errors ... . . . . . 12

2. One step improvement estimator . . . . . . . . . . 16

3. Maximum empirical likelihood estimator . . . . . . . . 17

C. Simulation results for estimating the parameter . . . . . 18

III $\square$ ESTIMATING THE MEAN RESPONSE IN REGRESSION . 21

A. Listwise deletion . . . . . . . . . . . . . . . 21

B. Propensity score . . . . . . . . . . . . . . . . 25

C. Partial imputation . . . . . . . . . . . . . . 27

D. Eull imputation . . . . . . . . . . . . . 40

IV COMPARISON OF PARTIAL AND FULL IMPUTATION .. . 44

A. Efficient estimate for $\vartheta \ldots \ldots \ldots \ldots$

B. Weighted least squares estimate of $\vartheta \ldots . . . . . . .4 .48$

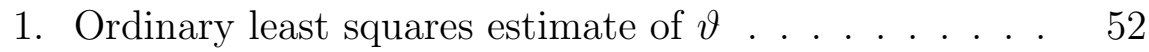

2. Constant weight for WLS .. . . . . . . . . . . 54

3. A poor choice of weights in WLS ... . . . . . . 55

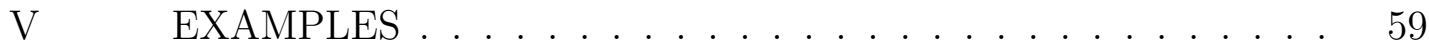

A. Symmetric missing structure . . . . . . . . . . . . 60

B. Gaussian missing structure . . . . . . . . . . . . . . . 63

C. Exponential missing structure . . . . . . . . . . . . 65

D. Simulation results with finite sample sizes _. . . . . . . 68 
VI SUMMARY $\ldots \ldots \ldots \ldots \ldots \ldots \ldots \ldots$

REFERENCES . . . . . . . . . . . . . . . . . . 74

APPENDIX . . . . . . . . . . . . . . . . . . 77

A. Additional results and tables of MSE . . . . . . . . . 78

1. Estimation of $\theta \ldots \ldots \ldots \ldots$

2. Estimation of $E(Y) \ldots \ldots \ldots \ldots \ldots$

a. No missing data . . . . . . . . . . . . . . . 83

b. Gaussian missing structure . . . . . . . . . . . . 93

c. Exponential missing structure . . . . . . . . . . . 104

B. $\mathrm{R}$ code . . . . . . . . . . . . . . . . . . . . . 113

1. Simulations with calculation of $\vartheta$ and $\mathrm{E}(\mathrm{Y})$. . . . 113

2. Combine output files . . . . . . . . . . . . . 126

3. Graph the MSE for the estimation of $\vartheta$. . . . . . . . 131

4. Create a table for the estimation of $\vartheta$. . . . . . . . 134

5. Solve for the MSE of the propensity score method . . 139

6. Graphs of the $\mathrm{E}(Y) \ldots \ldots \ldots \ldots$. . . . . . . . 141

7. Tables of MSE values for estimating $\mathrm{E}(\mathrm{Y})$. . . . . . . 145

8. The asymptotic variances of estimators for $E(Y)$. . 150

C. Semi-parametric regression . . . . . . . . . . . . . . . 158

1. Introduction . . . . . . . . . . . . . . 158

2. Perturbations through Hellinger derivatives . . . . . . 159

3. Hajek-Le Cam convolution theorem . . . . . . . . . . 164

4. Simplifying the tangent space . . . . . . . . . . . . . 172

5. Solving for the canonical gradient . . . . . . . . . . 182

VITA . . . . . . . . . . . . . . . . . . . . . . . . . . . . . . 195 


\section{LIST OF TABLES}

TABLE

Page

I The variance and Fisher's information for various error distributions.

II The asymptotic variances where the missing structure is symmetric. .

III The asymptotic variances where the missing structure is Gaussian. .

IV The asymptotic variances where the missing structure is exponential.

V Simulation results for the estimation of $\vartheta$ from 20,000 iterations. . . .

VI Simulation results showing the MSE for the estimation of $E[Y]$ where
there is no missing data and the errors have a normal distribution . . 88

VII Simulation results showing the MSE for the estimation of $E[Y]$ where there is no missing data and the errors have a $t_{2}$ distribution . . . . .

VIII Simulation results showing the MSE for the estimation of $E[Y]$ where there is no missing data and the errors have a logistic distribution . . 90

IX Simulation results showing the MSE for the estimation of $E[Y]$ where there is no missing data and the errors have a Gumbell distribution . 91

$\mathrm{X} \quad$ Simulation results showing the MSE for the estimation of $E[Y]$ where there is no missing data and the errors have a gamma distribution. . 92

XI Simulation results showing the MSE for the estimation of $E[Y]$ where the missing structure is Gaussian and the errors have a normal

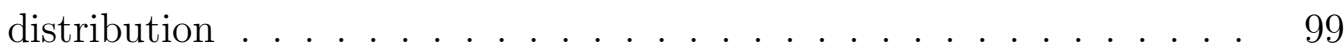


XII Simulation results showing the MSE for the estimation of $E[Y]$ where the missing structure is Gaussian and the errors have a $t_{2}$ distribution 100

XIII Simulation results showing the MSE for the estimation of $E[Y]$ where the missing structure is Gaussian and the errors have a logistic distribution . . . . . . . . . . . . . . . . . . . . . 101

XIV Simulation results showing the MSE for the estimation of $E[Y]$ where the missing structure is Gaussian and the errors have a Gumbel distribution . . . . . . . . . . . . . . . . . 102

XV Simulation results showing the MSE for the estimation of $E[Y]$ where the missing structure is Gaussian and the errors have a gamma distribution . . . . . . . . . . . . . . . . . 103

XVI Simulation results showing the MSE for the estimation of $E[Y]$ where the missing structure is exponential and the errors have a normal distribution . . . . . . . . . . . . . . . . . 108

XVII Simulation results showing the MSE for the estimation of $E[Y]$ where the missing structure is exponential and the errors have a $t_{2}$ dis-

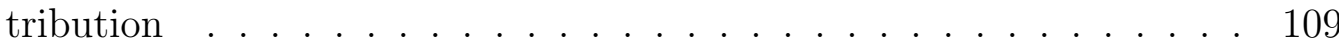

XVIII Simulation results showing the MSE for the estimation of $E[Y]$ where the missing structure is exponential and the errors have a logistic distribution . . . . . . . . . . . . . . . . . 110 
TABLE

Page

XIX Simulation results showing the MSE for the estimation of $E[Y]$ where the missing structure is exponential and the errors have a Gumbel distribution . . . . . . . . . . . . . . . . . . 111

XX Simulation results showing the MSE for the estimation of $E[Y]$ where the missing structure is exponential and the errors have a gamma distribution . . . . . . . . . . . . . . . . . . 112 


\section{LIST OF FIGURES}

FIGURE $\quad$ Page

$1 \quad$ MSE for various methods of estimating $\vartheta$ under $t_{2}$ errors. . . . . . 20

$2 \quad$ MSE for various methods of estimating $\vartheta$ under gamma errors. . . . 20

$3 \quad$ Symmetric missing structure. The missingness is centered over

$E(X)=1$ and is stepwise. $\ldots \ldots \ldots \ldots \ldots \ldots \ldots$

$4 \quad$ Gaussian missing structure. . . . . . . . . . . . . . . . 64

$5 \quad$ Exponential missing structure $\ldots \ldots \ldots \ldots 6$

$6 \quad$ MSE for estimating $E[Y]$ under normal errors with no missing data . 69

$7 \quad$ MSE for estimating $E[Y]$ under normal errors with an exponential

missing structure $\ldots \ldots \ldots \ldots \ldots \ldots$. . . . . . . . . . . . . .

$8 \quad$ MSE for estimating $E[Y]$ under gamma errors with an exponential

missing structure $\ldots \ldots \ldots \ldots \ldots \ldots \ldots$. . . . . . . . . . . . .

$9 \quad$ MSE for various methods of estimating $\vartheta$ under normal errors. . . . . 79

$10 \quad$ MSE for various methods of estimating $\vartheta$ under logistic errors. . . . . 79

$11 \quad$ MSE for various methods of estimating $\vartheta$ under Gumbel errors. . . . 80

$12 \quad$ MSE for various methods of estimating $\vartheta$ under double exponen-

tial errors. . . . . . . . . . . . . . . . . . 80 
$13 \quad$ MSE for estimating $E[Y]$ where the errors have the t distribution and no missing data . . . . . . . . . . . . . . . . . . . 84

$14 \quad$ MSE for estimating $E[Y]$ where the errors have the gamma distribution and no missing data . . . . . . . . . . . . . . . . 85

$15 \quad$ MSE for estimating $E[Y]$ where the errors have the logistic distribution and no missing data . . . . . . . . . . . . . . . . 86

$16 \quad$ MSE for estimating $E[Y]$ where the errors have the Gumbel distribution and no missing data . . . . . . . . . . . . . . . . . . . 87

17 MSE for estimating $E[Y]$ where the errors have the normal distribution and a Gaussian missingness structure . . . . . . . . . . . . 94

$18 \quad$ MSE for estimating $E[Y]$ where the errors have the t distribution and a Gaussian missingness structure . . . . . . . . . . . . . . . . . . 95

19 MSE for estimating $E[Y]$ where the errors have the gamma distribution and a Gaussian missingness structure . . . . . . . . . . . . 96

$20 \quad$ MSE for estimating $E[Y]$ where the errors have the logistic distribution and a Gaussian missingness structure . . . . . . . . . . . . 97

$21 \quad$ MSE for estimating $E[Y]$ where the errors have the Gumbel distribution and a Gaussian missingness structure . . . . . . . . . . . . 98

$22 \quad$ MSE for estimating $E[Y]$ where the errors have the t distribution and an exponential missing structure . . . . . . . . . . . . . . . . 105 
FIGURE

$23 \quad$ MSE for estimating $E[Y]$ where the errors have the logistic distribution and an exponential missing structure. . . . . . . . . . 106

$24 \quad$ MSE for estimating $E[Y]$ where the errors have the Gumbel distribution and an exponential missing structure . . . . . . . . . . . . . 107 


\section{LIST OF THEOREMS, LEMMAS, AND COROLLARIES}

Lemma II.1 . . . . . . . . . . . . . . . . . . . . . . . . . . . . 6

$$
\begin{aligned}
b\left(\delta_{i}, X_{i}, \epsilon_{i}\right)=E(\delta \quad & {\left[\{X-E(X \mid \delta=1)\} l(\epsilon)+E(X \mid \delta=1) \frac{\epsilon}{\sigma^{2}}\right] } \\
& {\left.\left[\{X-E(X \mid \delta=1)\} l(\epsilon)+E(X \mid \delta=1) \frac{\epsilon}{\sigma^{2}}\right]^{\top}\right)^{-1} } \\
& \delta_{i}\left[\left\{X_{i}-E(X \mid \delta=1)\right\} l\left(\epsilon_{i}\right)+E(X \mid \delta=1) \frac{\epsilon_{i}}{\sigma^{2}}\right] .
\end{aligned}
$$

Lemma II.2

$$
n^{1 / 2}(\hat{\vartheta}-\vartheta)=n^{-1 / 2} E\left[\delta X X^{\top}\right]^{-1} \sum_{i=1}^{n} \delta_{i} X_{i} \epsilon_{i}+o_{p}(1)
$$

Lemma II.3 . . . . . . . . . . . . . . . . . . . . . . . . . .

$$
\left[E\left[\delta X X^{\top}\right]^{-1}-n\left[\sum_{i=1}^{n} \delta_{i} X_{i} X_{i}^{\top}\right]^{-1}\right] \frac{1}{\sqrt{n}} \sum_{i=1}^{n}\left(\delta_{i} X_{i} \epsilon_{i}-E[\delta X \epsilon]\right)=o_{p}(1) .
$$

\section{Theorem II.4}

$$
\sqrt{n}\left(\hat{\vartheta}_{O L S}-\vartheta\right)=n^{-1 / 2} \sum_{i=1}^{n} b\left(X_{i}, \delta_{i} Y_{i}, \delta_{i}\right)+o_{p}(1)
$$

Lemma II.5 . . . . . . . . . . . . . . . . . . . . . . . . . . . . .

$$
\begin{aligned}
b\left(\delta_{i}, X_{i}, \epsilon_{i}\right)= & E\left[\delta\left(X-\frac{1}{2} E[X \mid \delta=1]\right)\left(X-\frac{1}{2} E[X \mid \delta=1]\right)^{\top}\right. \\
& \left.+\frac{1}{4} \delta E[X \mid \delta=1] E[X \mid \delta=1]^{\top}\right]^{-1} \\
& \left(\operatorname{sign}\left(\epsilon_{i}\right) \delta_{i} \lambda\left(X_{i}-E[X \mid \delta=1]\right)+\frac{1}{2} \delta_{i} \epsilon_{i} E[X \mid \delta=1]\right) .
\end{aligned}
$$




$$
\frac{M S E\left(\hat{\vartheta}_{E F F}\right)}{M S E\left(\hat{\vartheta}_{O L S}\right)}=\frac{1}{2} .
$$

Theorem III.1 . . . . . . . . . . . . . . . . . . . . . . . . . . .

When the missing structure is symmetric over symmetric covariates

$$
E(\delta X)=E(\delta) E(X)
$$

Theorem III.2 . . . . . . . . . . . . . . . . . . . . . . . . . . . . . . . . . . . 28

$$
E\left\{\widehat{E(Y)_{P I}}\right\} \doteq E(Y)
$$

Theorem III.3 . . . . . . . . . . . . . . . . . . . . . . . . . .

$$
\begin{aligned}
A V_{P I}= & \vartheta^{\top}\left\{E\left(X X^{\top}\right)-E(X) E(X)^{\top}\right\} \vartheta+\sigma^{2} E(\delta) \\
& +2 E(b \delta \epsilon)^{\top}\{E(X)-E(\delta X)\}-2 \vartheta^{\top} E(\delta X) E\left(\delta X^{\top} b\right) \\
& -2 \vartheta^{\top} E\left(\delta X b^{\top}\right)\{E(X)-E(\delta X)\}+2 \vartheta^{\top} E\left(X b^{\top}\right) E(X) \\
& +\{E(X)-E(\delta X)\}^{\top} E\left(b b^{\top}\right)\{E(X)-E(\delta X)\} .
\end{aligned}
$$

Theorem III.4 . . . . . . . . . . . . . . . . . . . . . . . . .

$$
E\left(\widehat{E(Y)_{F I}}\right)=E(Y)
$$

Theorem III.5 . . . . . . . . . . . . . . . . . . . . . . . .

$$
\begin{aligned}
A V_{F I}= & \vartheta^{\top}\left\{E\left(X X^{\top}\right)-E(X) E(X)^{\top}\right\} \vartheta+2 \vartheta^{\top} E\left(X b^{\top}\right) E(X) \\
& +2 \vartheta^{\top} E(X) E\left(X^{\top} b\right)+E(X)^{\top} E\left(b b^{\top}\right) E(X) .
\end{aligned}
$$

Theorem IV.3 . . . . . . . . . . . . . . . . . . . . . .

$$
\begin{aligned}
& n^{1 / 2}(\hat{\vartheta}-\vartheta) \\
= & \left\{E\left(\delta w_{\vartheta}(X) X^{\top}\right)\right\}^{-1} n^{-1 / 2} \sum_{i=1}^{n} \delta_{i} w_{\vartheta}\left(X_{i}\right)\left(Y_{i}-\vartheta^{\top} X_{i}\right)+o_{p}(1) .
\end{aligned}
$$

Lemma VI.1 . . . . . . . . . . . . . . . . . . . 


$$
\dot{P}_{n}=\{u(X, Z)+\delta v(Y, X, Z)+(\delta-\pi) w(X, Z)\} .
$$

Lemma VI.2

$$
\begin{aligned}
& E\{Y u(X, Z)\}+E\{Y v(Y, X, Z)\} \\
= & E\left\{u_{*}(X, Z) u(X, Z)\right\}+E\left\{\delta v_{*}(Y, X, Z) v(Y, X, Z)\right\} \\
+ & E\left[\{\delta-\pi(X, Z)\}^{2} w_{*}(X, Z) w(X, Z)\right] .
\end{aligned}
$$

Lemma VI.3 . . . . . . . . . . . . . . . . . . . . 167

$$
\begin{aligned}
w_{*}(X, Z) & =0 \\
g r_{\left(\vartheta_{*}, \gamma_{*}\right)} & =u_{*}(X, Z)+\delta v_{*}(Y, X, Z) .
\end{aligned}
$$

$$
\begin{aligned}
u_{*}(X, Z) & =r_{\vartheta}(X)+\gamma(Z)-E\left\{r_{\vartheta}(X)+\gamma(Z)\right\} \\
g r_{\left(\vartheta_{*}, \gamma_{*}\right)} & =r_{\vartheta}(X)+\gamma(Z)-E\left\{r_{\vartheta}(X)+\gamma(Z)\right\}+\delta v_{*}(Y, X, Z) .
\end{aligned}
$$

Lemma VI.5 . . . . . . . . . . . . . . . . . . . . . . 170

$$
v_{*}(Y, X, Z)=s(\epsilon)+l(\epsilon)\left\{t^{\top} X+g(Z)\right\} .
$$

Lemma VI.6 . . . . . . . . . . . . . . . . . . . 173

$$
E[g(X, \delta) \epsilon]=0
$$

Lemma VI.7 . . . . . . . . . . . . . . . . . . . . . . . . . . . . . . . 173

$$
E\{\epsilon l(\epsilon)\}=1 \text {. }
$$

Lemma VI.8 . . . . . . . . . . . . . . . . . . . . . . . . . . . . . . . . . . . . . 174

$$
E\{\delta g(X, Z, \delta, Y)\}=E(\delta) E\{g(X, Z, \delta, Y) \mid \delta=1\}
$$

Lemma VI.9 . . . . . . . . . . . . . . . . . . . . . . . . . . . . . . 175 


$$
s_{2}(\epsilon)+\xi(X, Z, \epsilon)
$$

where

$$
\begin{aligned}
s_{2}(\epsilon) & \in s(\epsilon) \\
\xi(X, Z, \epsilon) & =[\phi(X, Z)-E\{\phi(X, Z) \mid \delta=1\}] l(\epsilon)+E\{\phi(X, Z) \mid \delta=1\} \frac{\epsilon}{\sigma^{2}} \\
\phi(X, Z) & =t^{\top} \dot{r}_{\vartheta}(X)+g(Z)
\end{aligned}
$$

Lemma VI.10 . . . . . . . . . . . . . . . . . . . . . . . . . . . . . . . . . 178

$$
E\{\phi(X, Z)\}=E\left[\delta\left\{s_{2 *}(\epsilon)+\xi_{*}(X, Z, \epsilon)\right\}\left\{s_{2}(\epsilon)+\xi(X, Z, \epsilon)\right\}\right] .
$$

Lemma VI.11 . . . . . . . . . . . . . . . . . . . . . . . . . 180

$$
v_{*}(Y, X, Z)=\xi_{*}(X, Z, \epsilon) .
$$

Corollary VI.12 . . . . . . . . . . . . . . . . . . . . 181

$$
E\{\phi(X, Z)\}=E\left\{\delta \xi_{*}(X, Z, \epsilon) \xi(X, Z, \epsilon)\right\}
$$

Lemma VI.13 . . . . . . . . . . . . . . . . . . . . . . . . . . . . . . . 182

$$
E\left\{\delta \phi_{*}(X, Z)\right\}=\sigma^{2}
$$

Corollary VI.14 . . . . . . . . . . . . . . . . . . . . . . . . . 184

$$
\xi_{*}(X, Z, \epsilon)=\phi_{*}(X, Z) l(\epsilon)-\frac{\sigma^{2}}{E(\delta)} l(\epsilon)+\frac{\epsilon}{E(\delta)}
$$

Lemma VI.15 . . . . . . . . . . . . . . . . . . . . . . 185

$$
E\left\{\delta l(\epsilon) \xi_{*}(X, Z, \epsilon)\right\}=1
$$

Lemma VI.16 . . . . . . . . . . . . . . . . . . . . . . . . . 185

$$
E\left\{\delta \epsilon \xi_{*}(X, Z, \epsilon)\right\}=\sigma^{2}
$$


Lemma VI.17 . . . . . . . . . . . . . . . . . . . . . . 186

$$
\begin{aligned}
E\left\{\delta \phi(X, Z) l(\epsilon) \xi_{*}(X, Z, \epsilon)\right\}= & E\left\{\delta \phi(X, Z) \phi_{*}(X, Z)\right\} \mathbb{I}-\frac{\sigma^{2}}{E(\delta)} E\{\delta \phi(X, Z)\} \mathbb{I} \\
& +\frac{E\{\delta \phi(X, Z)\}}{E(\delta)} .
\end{aligned}
$$

Lemma VI.18 . . . . . . . . . . . . . . . . . . . . . . . . . . . . . 186

$$
g_{*}(Z)=\frac{\sigma^{2}}{E(\delta)}-\frac{1}{E(\delta) \mathbb{I}}+\frac{1}{E(\delta \mid Z) \mathbb{I}}-t_{*}^{\top} \frac{E\left\{\delta \dot{r}_{\vartheta}(X) \mid Z\right\}}{E(\delta \mid Z)} .
$$

Lemma VI.19 . . . . . . . . . . . . . . . . . . . . . . . . . . . . . . . . 189

$$
\begin{aligned}
t_{*}= & \frac{1}{\mathbb{I}}\left(E\left\{\delta \dot{r}_{\vartheta}(X) \dot{r}_{\vartheta}(X)^{\top}\right\}-E\left[\frac{E\left\{\delta \dot{r}_{\vartheta}(X) \mid Z\right\} E\left\{\delta \dot{r}_{\vartheta}(X) \mid Z\right\}^{\top}}{E(\delta \mid Z)}\right]\right)^{-1} \\
& {\left[E\left\{\dot{r}_{\vartheta}(X)\right\}-\frac{E\left\{\delta \dot{r}_{\vartheta}(X) \mid Z\right\}}{E(\delta \mid Z)}\right] . }
\end{aligned}
$$

Lemma VI.20 . . . . . . . . . . . . . . . . . . . . . . . . . . . . . 191

$$
\begin{aligned}
v_{*}(Y, X, Z)= & \frac{\epsilon}{E(\delta)}-\frac{l(\epsilon)}{E(\delta) \mathbb{I}}+\frac{l(\epsilon)}{E(\delta \mid Z) \mathbb{I}}-\frac{l(\epsilon)}{\mathbb{I}}\left(E\left\{\dot{r}_{\vartheta}(X)^{\top}\right\}-E\left[\frac{E\left\{\delta \dot{r}_{\vartheta}(X) \mid Z\right\}}{E(\delta \mid Z)}\right]\right) \\
& \left(E\left\{\delta \dot{r}_{\vartheta}(X) \dot{r}_{\vartheta}(X)^{\top}\right\}-E\left[\frac{E\left\{\delta \dot{r}_{\vartheta}(X) \mid Z\right\} E\left\{\delta \dot{r}_{\vartheta}(X) \mid Z\right\}^{\top}}{E(\delta \mid Z)}\right]\right)^{-1} \\
& {\left[\dot{r}_{\vartheta}(X)-\frac{E\left\{\delta \dot{r}_{\vartheta}(X) \mid Z\right\}}{E(\delta \mid Z)}\right] . }
\end{aligned}
$$

Theorem VI.21 $\ldots \ldots+\cdots \cdots$ 


$$
\begin{aligned}
g r_{\left(\vartheta_{*}, \gamma_{*}\right)}= & r_{\vartheta}(X)+\gamma(Z)-E\left\{r_{\vartheta}(X)+\gamma(Z)\right\}+\frac{\delta \epsilon}{E(\delta)}-\frac{\delta l(\epsilon)}{E(\delta) \mathbb{I}}+\frac{\delta l(\epsilon)}{E(\delta \mid Z) \mathbb{I}} \\
& -\frac{\delta l(\epsilon)}{\mathbb{I}}\left(E\left\{\dot{r}_{\vartheta}(X)^{\top}\right\}-E\left[\frac{E\left\{\delta \dot{r}_{\vartheta}(X) \mid Z\right\}}{E(\delta \mid Z)}\right]\right) \\
& \left(E\left\{\delta \dot{r}_{\vartheta}(X) \dot{r}_{\vartheta}(X)^{\top}\right\}-E\left[\frac{E\left\{\delta \dot{r}_{\vartheta}(X) \mid Z\right\} E\left\{\delta \dot{r}_{\vartheta}(X) \mid Z\right\}^{\top}}{E(\delta \mid Z)}\right]\right)^{-1} \\
& {\left[\dot{r}_{\vartheta}(X)-\frac{E\left\{\delta \dot{r}_{\vartheta}(X) \mid Z\right\}}{E(\delta \mid Z)}\right] . }
\end{aligned}
$$




\section{CHAPTER I}

\section{INTRODUCTION}

This work examines methods to efficiently estimate the mean response in a semiparametric model under the assumption that the responses are missing at random. A study by Elliot (2008) illustrates the complexity of such a problem. He investigated the link between specific minority groups (e.g., non-Mexican Hispanic Americans or Chinese Americans) and obesity in children. The response variable, weight of the child, was frequently missing and laws restricting personal information made it impossible to recover the missing data. Because the missing structure was correlated with other covariates (e.g. height of the child and location) in the model the results would have been biased without imputation, i.e. without estimation of the missing values.

Schick (1993) explains how efficient estimators are formed for regression models when no distributional assumptions are made on the covariates. The Statistical analysis with missing data book by Little and Rubin (2002) is well known for its explanation on the estimation of regression parameters under the assumption of data missing at random. Müller et al. (2006) propose the method of full imputation, which estimates all the responses, as an improvement over partial imputation, where only the missing responses are imputed. Müller (2009) showed that in order to efficiently estimate any function of the response it is required to estimate the regression parameters efficiently.

We begin by investigating efficient estimation of the regression parameter when the error distribution is unknown. The Ordinary Least Squares method is proven to be efficient when the error distribution happens to be normal. The complete case

The journal model is The American Statistician. 
versions of the One Step Improvement Estimator discussed in Forrester et al. (2003) and the Maximum Empirical Likelihood Estimator discussed in Peng and Schick (2012) are presented as efficient estimators regardless of the error distribution. We use simulations to show the mean square error of these estimators under various distributions.

To estimate the mean response with missing data we compare four common methods: Listwise Deletion, Propensity Score method, Partial Imputation, and Full Imputation. We also derive the asymptotic variances for each method. Simulation results show the MSE of the estimate of the mean response under various error distributions. We show how the MSE is affected by the method of imputation and by the estimator of the regression parameter.

This research illustrates the impact that the imputation method has on estimation in regression models that have missing data. Full imputation is shown to have the least asymptotic variance when the parameter is estimated efficiently. With an inefficient estimate of the parameter we see that full imputation can have more asymptotic variance than partial imputation. When the missing structure is not symmetric about the covariate, listwise deletion methods will be biased. We find some non-regular errors where the OLS estimator for the regression parameter performs better than efficient estimators. The simulations show how each estimator performs for finite sample sizes.

The paper is organized as follows: Chapter II investigates efficient estimation of the regression parameter. Chapter III shows the asymptotic variance for different methods we use to estimate the mean response with missing data. In Chapter [V] we study estimation of the mean response. We compare the asymptotic variance of the partially imputed estimator to the fully imputed estimator. In Chapter $\mathrm{V}$ we show the asymptotic variances for the imputation methods under various scenarios. Our 
conclusions are in Chapter VI.

Appendix A shows results from simulations using additional models not previously shown. Additionally there are tables of the simulated values for the MSE of estimating $\vartheta$ and $E(Y)$. Appendix B contains the $\mathrm{R}$ code used to solve equations, create graphs, and run the simulations. Appendix C is an extension of the model given in the paper to a more general semi-parametric model. The method to find the canonical gradient is shown, but no estimator has yet been proven to have an influence function that matches the canonical gradient. 


\section{CHAPTER II}

\section{PARAMETER ESTIMATION IN LINEAR REGRESSION}

A. The model

The model form is

$$
Y=\vartheta^{\top} X+\epsilon
$$

where $\vartheta$ is assumed to be fixed but unknown, the covariates, $X$, and errors, $\epsilon$, are assumed to have a random but unknown distribution. The distribution of $\epsilon, f(\epsilon)$, has a mean of zero and finite variance $\sigma^{2}$. Further assume that $X$ is square-integrable with finite second moments and independent of $\epsilon$. The expected value of $\epsilon$ is 0 with variance $\sigma^{2}$. The observed variables are $(X, \delta, \delta Y)$ where $\delta$ is 0 if the response, $Y$, is not observed, and 1 if the response is observed.

The conditional probability is assumed to depend only on the covariate, not the response, meaning

$$
P(\delta=1 \mid X, Y)=P(\delta=1 \mid X)=E[\delta \mid X]=\pi(X)
$$

This is called the Missing At Random (MAR) assumption.

The model is studied in Müller et al. (2006). The joint probability of $d x, d y$, and $d \delta, P(d x, d y, d \delta)$ is defined by

$$
P(d x, d y, d \delta)=G(d x) B_{\pi(X)}(d \delta)\left\{\delta Q(x, d y)+(1-\delta) \delta_{o}(d y)\right\}
$$

where $G(d x)$ is the marginal distribution on $d x, B_{\pi(X)}(d \delta)$ is the Bernoulli distribution with conditional probability $\pi(X)=P(\delta=1 \mid X), Q$ is the conditional distribution of 
$d y$ given $X$, and $\delta_{o}(d y)$ is the Dirac measure at 0 .

This work is based on the Hajek-Le Cam theory for locally asymptotically normal families. An estimator of some function $k(G, Q, \pi)$, call it $\hat{k}(G, Q, \pi)$, is asymptotically efficient if it is asymptotically linear, meaning

$$
n^{1 / 2}\{\hat{k}(G, Q, \pi\}-k(G, Q, \pi))=n^{-1 / 2} \sum_{i=1}^{n} b\left(X_{i}, \epsilon_{i}, \delta_{i}\right)+o_{p}(1),
$$

and if $b\left(X_{i}, \delta_{i} Y_{i}, \delta_{i}\right) \in L_{2,0}(P)$ is the efficient influence function.

\section{B. Estimating the parameter}

In this section it will be shown that the parameter $\vartheta$ in the linear model can be found efficiently, and in Subsection 1 it will be shown that when the unknown error distribution is normal the efficient estimator matches the Ordinary Least Squares estimator. A One Step Improvement estimator is proposed in Subsection 2 and a Maximum Empirical Likelihood estimator is proposed in Subsection 3 which are efficient even when the error distribution is not in fact normal. In Section C simulations are used to find the MSE of each estimator for $\vartheta$ under various scenarios. The graphs show how the efficient estimators outperform the inefficient ones for various sample sizes.

To find the influence function for an estimator of the parameter in a parametric model we refer to the work of Müller (2009). Define the parametric model as $Y=$ $r_{\vartheta}(X)+\epsilon$ where $r_{\vartheta}(X)$ has derivative $\dot{r}_{\vartheta}(X)$, and the score function of $\epsilon$ is $l(\epsilon)$. Define

$$
\zeta\left(\delta_{i}, X_{i}, \epsilon_{i}\right)=\left[\dot{r}_{\vartheta}\left(X_{i}\right)-E\left\{\dot{r}_{\vartheta}(X) \mid \delta=1\right\}\right] l\left(\epsilon_{i}\right)+E\left\{\dot{r}_{\vartheta}(X) \mid \delta=1\right\} \frac{\epsilon_{i}}{\sigma^{2}} .
$$

The efficient influence function for the parameter $\vartheta$ is

$$
b\left(\delta_{i}, X_{i}, \epsilon_{i}\right)=E\left\{\delta \zeta(\delta, X, \epsilon) \zeta(\delta, X, \epsilon)^{\top}\right\}^{-1} \delta_{i} \zeta\left(\delta_{i}, X_{i}, \epsilon_{i}\right)
$$


This influence function holds for any parametric model of $Y$.

Next we will prove two lemmas. The first lemma shows the influence function under the assumption of linear regression. The second lemma shows the influence function under the assumption of linear regression and normally distributed errors.

Lemma II.1 Using the model defined above, the influence function for the linear regression model where $r_{\vartheta}(X)=\vartheta^{\top} X$ is

$$
\begin{aligned}
b\left(\delta_{i}, X_{i}, \epsilon_{i}\right)=E[\delta \quad & {\left[\{X-E(X \mid \delta=1)\} l(\epsilon)+E(X \mid \delta=1) \frac{\epsilon}{\sigma^{2}}\right] } \\
& \left.\left\{[X-E(X \mid \delta=1)] l(\epsilon)+E(X \mid \delta=1) \frac{\epsilon}{\sigma^{2}}\right\}^{\top}\right]^{-1} \\
& \delta_{i}\left\{\left[X_{i}-E(X \mid \delta=1)\right] l\left(\epsilon_{i}\right)+E(X \mid \delta=1) \frac{\epsilon_{i}}{\sigma^{2}}\right\} .
\end{aligned}
$$

Proof: Assuming $r_{\vartheta}(X)=\vartheta^{\top} X$ then $\dot{r}_{\vartheta}(X)=X$, and Equation 2.2 becomes

$$
\zeta\left(\delta_{i}, X_{i}, \epsilon_{i}\right)=\{X-E[X \mid \delta=1]\} l\left(\epsilon_{i}\right)+E[X \mid \delta=1] \frac{\epsilon_{i}}{\sigma^{2}}
$$

The efficient influence function from Equation 2.3 is

$$
\begin{aligned}
b\left(\delta_{i}, X_{i}, \epsilon_{i}\right)=E[\delta \quad & \left\{[X-E(X \mid \delta=1)] l(\epsilon)+E(X \mid \delta=1) \frac{\epsilon}{\sigma^{2}}\right\} \\
& \left.\left\{[X-E(X \mid \delta=1)] l(\epsilon)+E(X \mid \delta=1) \frac{\epsilon}{\sigma^{2}}\right\}^{\top}\right]^{-1} \\
& \delta_{i}\left\{\left[X_{i}-E(X \mid \delta=1)\right] l\left(\epsilon_{i}\right)+E(X \mid \delta=1) \frac{\epsilon_{i}}{\sigma^{2}}\right\} .
\end{aligned}
$$

Lemma II.2 Using the simple linear regression model when the distribution of the errors, $\epsilon$, is normally distributed an efficient estimator for $\vartheta$ will have the asymptotic 
form

$$
n^{1 / 2}(\hat{\vartheta}-\vartheta)=n^{-1 / 2} E\left[\delta X X^{\top}\right]^{-1} \sum_{i=1}^{n} \delta_{i} X_{i} \epsilon_{i}+o_{p}(1) .
$$

Proof: When the errors are normally distributed the score function will be $l(\epsilon)=\frac{\epsilon}{\sigma^{2}}$. So, by Lemma II.1, the influence function will be

$$
\begin{aligned}
b\left(\delta_{i}, X_{i}, \epsilon_{i}\right)= & E\left[\delta\left\{[X-E(X \mid \delta=1)] \frac{\epsilon}{\sigma^{2}}+E(X \mid \delta=1) \frac{\epsilon}{\sigma^{2}}\right\}\right. \\
& \left.\left\{[X-E(X \mid \delta=1)] \frac{\epsilon}{\sigma^{2}}+E(X \mid \delta=1) \frac{\epsilon}{\sigma^{2}}\right\}^{\top}\right]^{-1} \\
& \delta_{i}\left\{\left[X_{i}-E(X \mid \delta=1)\right] \frac{\epsilon_{i}}{\sigma^{2}}+E(X \mid \delta=1) \frac{\epsilon_{i}}{\sigma^{2}}\right\} \\
= & E\left[\delta X \frac{\epsilon}{\sigma^{2}} X^{\top} \frac{\epsilon}{\sigma^{2}}\right]^{-1} \delta_{i} X_{i} \frac{\epsilon_{i}}{\sigma^{2}} \\
= & E\left[\delta X X^{\top} \epsilon^{2}\right]^{-1} \sigma^{2} \delta_{i} X_{i} \epsilon_{i} \\
= & E\left[\delta X X^{\top}\right]^{-1} E\left[\epsilon^{2}\right]^{-1} \sigma^{2} \delta_{i} X_{i} \epsilon_{i} \\
= & E\left[\delta X X^{\top}\right]^{-1} \delta_{i} X_{i} \epsilon_{i} .
\end{aligned}
$$

Putting this influence function into Equation 2.1 with $k(G, Q, \pi)=\vartheta$ gives

$$
\begin{aligned}
n^{1 / 2}(\hat{\vartheta}-\vartheta) & =n^{-1 / 2} \sum_{i=1}^{n} E\left[\delta X X^{\top}\right]^{-1} \delta_{i} X_{i} \epsilon_{i}+o_{p}(1) \\
& =n^{-1 / 2} E\left[\delta X X^{\top}\right]^{-1} \sum_{i=1}^{n} \delta_{i} X_{i} \epsilon_{i}+o_{p}(1) .
\end{aligned}
$$

\section{Ordinary least squares estimator}

In this section we derive the OLS estimator for the missing data model, and introduce a theorem that the OLS estimator is efficient if $\epsilon$ is normally distributed. The model under consideration is $Y=\vartheta^{\top} X+\epsilon$ where $X$ is i.i.d. with finite variance and independent of $\epsilon$. Also assume $\delta$ is i.i.d. and independent of $\epsilon$ and that $Y$ is square 
integrable, and $\sum_{i=1}^{n} \delta_{i} X_{i} X_{i}^{\top}$ is invertible. To start we will introduce a term that is $o_{p}(1)$ which we will need later.

Lemma II.3 Using the model and notation above

$$
\left[E\left[\delta X X^{\top}\right]^{-1}-n\left[\sum_{i=1}^{n} \delta_{i} X_{i} X_{i}^{\top}\right]^{-1}\right] \frac{1}{\sqrt{n}} \sum_{i=1}^{n}\left(\delta_{i} X_{i} \epsilon_{i}-E[\delta X \epsilon]\right)=o_{p}(1) .
$$

Proof: By the Weak Law of Large Numbers where $\delta_{i} X_{i} X_{i}^{\top}$ are i.i.d. with finite variance,

$$
\frac{1}{n}\left[\sum_{i=1}^{n} \delta_{i} X_{i} X_{i}^{\top}\right] \stackrel{p}{\rightarrow} E\left[\delta X X^{\top}\right]
$$

Note that $\delta_{i} X_{i} X_{i}^{\top}=\sum_{j=1}^{n} \delta_{i} X_{i j}^{2} \geq 0$ is positive semidefinite and $\sum \delta_{i} X_{i} X_{i}^{\top}$ is invertible by assumption, so by Slutsky's theorem

$$
n\left[\sum_{i=1}^{n} \delta_{i} X_{i} X_{i}^{\top}\right]^{-1} \stackrel{a . s .}{\longrightarrow} E\left[\delta X X^{\top}\right]^{-1}
$$

which can be written as

$$
E\left[\delta X X^{\top}\right]^{-1}-n\left[\sum_{i=1}^{n} \delta_{i} X_{i} X_{i}^{\top}\right]^{-1}=o_{p}(1)
$$


Using the assumption of MAR where $E[\delta Y \mid X]=E[\delta \mid X] E[Y \mid X]$

$$
\begin{aligned}
E[\delta X \epsilon] & =E\left[\delta X\left(Y-\vartheta^{\top} X\right)\right] \\
& =E[\delta X Y]-E\left[\delta X \vartheta^{\top} X\right] \\
& =E[X E(\delta Y \mid X)]-E\left[X \vartheta^{\top} X E(\delta \mid X)\right] \\
& =E[X E(\delta \mid X) E(Y \mid X)]-E\left[X \vartheta^{\top} X E(\delta \mid X)\right] \\
& =E\left[X \vartheta^{\top} X E(\delta \mid X)\right]-E\left[X \vartheta^{\top} X E(\delta \mid X)\right] \\
& =0 .
\end{aligned}
$$

Because $\delta_{i} X_{i} \epsilon_{i}$ is i.i.d. with expected value of zero, the Central Limit Theorem states the sum has a limiting normal distribution with a convergence rate of $\sqrt{n}$. This implies

$$
\sqrt{n} \frac{1}{n} \sum_{i=1}^{n} \delta_{i} X_{i} \epsilon_{i}=O_{p}(1) .
$$

Then combining Equations 2.4, 2.5, and 2.6

$$
\begin{aligned}
& {\left[E\left[\delta X X^{\top}\right]^{-1}-n\left(\sum_{i=1}^{n} \delta_{i} X_{i} X_{i}^{\top}\right)^{-1}\right] \frac{1}{\sqrt{n}} \sum_{i=1}^{n}\left(\delta_{i} X_{i} \epsilon_{i}-E[\delta X \epsilon]\right) } \\
= & o_{p}(1)\left(O_{p}(1)\right) \\
= & o_{p}(1)
\end{aligned}
$$

The Ordinary Least Squares estimator for this model, $\hat{\vartheta}$, minimizes the squared difference between $Y$ and $\hat{Y}=\hat{\vartheta}^{\top} X_{i}$. The equation to minimize is

$$
\sum_{i=1}^{n} \delta_{i}\left(Y_{i}-\hat{Y}_{i}\right)^{2}
$$


The solution is found by setting the derivative to zero

$$
0=\left.\frac{\partial}{\partial \vartheta}\left(\sum_{i=1}^{n} \delta_{i}\left(Y_{i}-\vartheta^{\top} X_{i}\right)^{2}\right)\right|_{\vartheta=\hat{\vartheta}_{O L S}}=-2 \sum_{i=1}^{n} \delta_{i}\left(Y_{i}-\hat{\vartheta}_{O L S}^{\top} X_{i}\right) X_{i}^{\top} .
$$

This implies

$$
\sum_{i=1}^{n} \delta_{i} Y_{i} X_{i}^{\top}=\hat{\vartheta}_{O L S}^{\top} \sum_{i=1}^{n} \delta_{i} X_{i} X_{i}^{\top}
$$

Solving for $\hat{\vartheta}_{O L S}$ and taking the transpose:

$$
\hat{\vartheta}_{O L S}=\left[\sum_{i=1}^{n} \delta_{i} X_{i} X_{i}^{\top}\right]^{-1} \sum_{i=1}^{n} \delta_{i} X_{i} Y_{i} .
$$

Theorem II.4 Let $Y=\vartheta^{\top} X+\epsilon$ where $X$ is i.i.d. and independent of $\epsilon$. Assume that $Y$ is square integrable, and that $\sum_{i=1}^{n} \delta_{i} X_{i} X_{i}^{\top}$ is invertible. If in fact the errors are normally distributed, $\epsilon \sim N\left(0, \sigma^{2}\right)$, then the OLS estimator $\hat{\vartheta}_{O L S}$ is asymptotically efficient for $\vartheta$. From Equation 2.1 this means

$$
\sqrt{n}\left(\hat{\vartheta}_{O L S}-\vartheta\right)=n^{-1 / 2} \sum_{i=1}^{n} b\left(X_{i}, \delta_{i} Y_{i}, \delta_{i}\right)+o_{p}(1) .
$$

Proof: The definition of the OLS estimator says

$$
\begin{aligned}
& \sqrt{n}\left(\hat{\vartheta}_{\text {OLS }}-\vartheta\right) \\
= & \sqrt{n}\left(\left[\sum_{i=1}^{n} \delta_{i} X_{i} X_{i}^{\top}\right]^{-1} \sum_{i=1}^{n} \delta_{i} X_{i} Y_{i}-\vartheta\right) \\
= & \sqrt{n}\left(\left[\sum_{i=1}^{n} \delta_{i} X_{i} X_{i}^{\top}\right]^{-1}\left[\sum_{i=1}^{n} \delta_{i} X_{i} Y_{i}\right]-\left[\sum_{i=1}^{n} \delta_{i} X_{i} X_{i}^{\top}\right]^{-1}\left[\sum_{i=1}^{n} \delta_{i} X_{i} X_{i}^{\top}\right] \vartheta\right) .
\end{aligned}
$$


This can be written as

$$
\begin{aligned}
& \sqrt{n}\left(\hat{\vartheta}_{O L S}-\vartheta\right) \\
= & \sqrt{n}\left(\left[\sum_{i=1}^{n} \delta_{i} X_{i} X_{i}^{\top}\right]^{-1}\left[\sum_{i=1}^{n} \delta_{i} X_{i}\left(Y_{i}-X_{i}^{\top} \vartheta\right)\right]\right) \\
= & \sqrt{n}\left(\left[\sum_{i=1}^{n} \delta X_{i} X_{i}^{\top}\right]^{-1}\left[\sum_{i=1}^{n} \delta_{i} X_{i} \epsilon_{i}\right]\right) .
\end{aligned}
$$

Now by Lemma II.3

$$
\begin{aligned}
\sqrt{n}\left(\hat{\vartheta}_{O L S}-\vartheta\right)= & {\left[E\left[\delta X X^{\top}\right]^{-1}-n\left(\sum_{i=1}^{n} \delta_{i} X_{i} X_{i}^{\top}\right)^{-1}\right] \frac{1}{\sqrt{n}} \sum_{i=1}^{n}\left(\delta_{i} X_{i} \epsilon_{i}-E[\delta X \epsilon]\right) } \\
& +\sqrt{n}\left[\left(\sum_{i=1}^{n} \delta X_{i} X_{i}^{\top}\right)^{-1} \sum_{i=1}^{n} \delta_{i} X_{i} \epsilon_{i}\right]+o_{p}(1) .
\end{aligned}
$$

This simplifies to

$$
\begin{aligned}
\sqrt{n}\left(\hat{\vartheta}_{O L S}-\vartheta\right)= & \frac{1}{\sqrt{n}} E\left(\delta X X^{\top}\right)^{-1} \sum_{i=1}^{n} \delta_{i} X_{i} \epsilon_{i} \\
& -\sqrt{n}\left(\sum_{i=1}^{n} \delta_{i} X_{i} X_{i}^{\top}\right)^{-1} \sum_{i=1}^{n} \delta_{i} X_{i} \epsilon_{i} \\
& -\sqrt{n}\left[E\left[\delta X X^{\top}\right]^{-1}-n\left(\sum_{i=1}^{n} \delta_{i} X_{i} X_{i}^{\top}\right)^{-1}\right] E[\delta X \epsilon] \\
& +\sqrt{n}\left(\sum_{i=1}^{n} \delta_{i} X_{i} X_{i}^{\top}\right)^{-1} \sum_{i=1}^{n} \delta_{i} X_{i} \epsilon_{i}+ \\
& +o_{p}(1)
\end{aligned}
$$

This implies

$$
\begin{aligned}
\sqrt{n}\left(\hat{\vartheta}_{O L S}-\vartheta\right) & =\frac{1}{\sqrt{n}} \sum_{i=1}^{n}\left(E\left[\delta X X^{\top}\right]\right)^{-1} \delta_{i} X_{i} \epsilon_{i}+0+o_{p}(1) \\
& =E\left[\delta X X^{\top}\right]^{-1} \frac{1}{\sqrt{n}} \sum_{i=1}^{n} \delta_{i} X_{i} \epsilon_{i}+o_{p}(1) .
\end{aligned}
$$


Using Equation 2.1

$$
\sqrt{n}\left(\hat{\vartheta}_{O L S}-\vartheta\right)=n^{-1 / 2} \sum_{i=1}^{n} b\left(X_{i}, \delta_{i} Y_{i}, \delta_{i}\right)+o_{p}(1)
$$

Therefore by Lemma II.2 $\hat{\vartheta}_{\text {ols }}$ is an efficient estimator for $\vartheta$.

a. OLS with double exponential errors

Consider the model $Y=\vartheta^{\top} X+\epsilon$ where $\epsilon$ has an unknown distribution, but is in fact a Double Exponential random variable, given by the pdf for $z \in \mathbb{R}$, and $\lambda>0$,

$$
f(z)=\frac{1}{2 \lambda} e^{-|z| / \lambda}
$$

Further assume $Y$ is MAR depending on $\delta$. Assume $X$ is i.i.d. and independent of $\epsilon$. Assume $\mathrm{Y}$ is square integrable and that $\sum_{i=1}^{n} \delta_{i} X_{i} X_{i}^{\top}$ is invertible. I will use the efficient influence function to show that the OLS estimator is not efficient for all distributions.

Lemma II.5 For the model desribed above where the distribution of $\epsilon$ is unknown, but is actually a Double Exponential random variable the influence function

$$
\begin{aligned}
b\left(\delta_{i}, X_{i}, \epsilon_{i}\right)= & E\left[\delta\left(X-\frac{1}{2} E[X \mid \delta=1]\right)\left(X-\frac{1}{2} E[X \mid \delta=1]\right)^{\top}\right. \\
& \left.+\frac{1}{4} \delta E[X \mid \delta=1] E[X \mid \delta=1]^{\top}\right]^{-1} \\
& \left(\operatorname{sign}\left(\epsilon_{i}\right) \delta_{i} \lambda\left(X_{i}-E[X \mid \delta=1]\right)+\frac{1}{2} \delta_{i} \epsilon_{i} E[X \mid \delta=1]\right) .
\end{aligned}
$$

Proof: As defined above let $f(\epsilon)$ be the distribution of $\epsilon$, and let $\dot{f}(\epsilon)$ be the deriva- 
tive, then the score function for the Double Exponential distribution is

$$
l(\epsilon)=-\frac{\dot{f}(\epsilon)}{f(\epsilon)}=-\frac{-\frac{\operatorname{sign}(\epsilon)}{2 \lambda^{2}} e^{-|\epsilon| / \lambda}}{\frac{1}{2 \lambda} e^{-|\epsilon| / \lambda}}=\operatorname{sign}(\epsilon) \frac{1}{\lambda} .
$$

Using $\sigma^{2}=2 \lambda^{2}$ in Lemma II.1 the influence function is

$$
\begin{aligned}
b\left(\delta_{i}, X_{i}, \epsilon_{i}\right)= & E\left[\delta\left([X-E(X \mid \delta=1)] \operatorname{sign}(\epsilon) \frac{1}{\lambda}+E[X \mid \delta=1] \frac{\epsilon}{2 \lambda^{2}}\right)\right. \\
& \left.\left([X-E(X \mid \delta=1)] \operatorname{sign}(\epsilon) \frac{1}{\lambda}+E[X \mid \delta=1] \frac{\epsilon}{2 \lambda^{2}}\right)^{\top}\right]^{-1} \\
& \delta_{i}\left(\left[X_{i}-E(X \mid \delta=1)\right] \operatorname{sign}\left(\epsilon_{i}\right) \frac{1}{\lambda}+E[X \mid \delta=1] \frac{\epsilon_{i}}{2 \lambda^{2}}\right) .
\end{aligned}
$$

Using the fact that $\operatorname{sign}(\epsilon)^{2}=1, E\left(\epsilon^{2}\right)=2 \lambda^{2}$ and $E\{\operatorname{sign}(\epsilon) \epsilon\}=\lambda$

$$
\begin{aligned}
b\left(\delta_{i}, X_{i}, \epsilon_{i}\right)= & E\left[\delta \left\{\frac{1}{\lambda^{2}} X X^{\top}-\frac{1}{\lambda^{2}} X E(X \mid \delta=1)^{\top}+\frac{1}{2 \lambda^{2}} X E(X \mid \delta=1)^{\top}\right.\right. \\
& -\frac{1}{\lambda^{2}} E(X \mid \delta=1) X^{\top}+\frac{1}{\lambda^{2}} E(X \mid \delta=1) E(X \mid \delta=1)^{\top} \\
& -\frac{1}{2 \lambda^{2}} E(X \mid \delta=1) E(X \mid \delta=1)^{\top}+\frac{1}{2 \lambda^{2}} E(X \mid \delta=1) X^{\top} \\
& \left.\left.-\frac{1}{2 \lambda^{2}} E(X \mid \delta=1) E(X \mid \delta=1)^{\top}+\frac{1}{2 \lambda^{2}} E(X \mid \delta=1) E(X \mid \delta=1)^{\top}\right\}\right]^{-1} \\
& \delta_{i}\left\{\operatorname{sign}\left(\epsilon_{i}\right) \frac{1}{\lambda} X-\operatorname{sign}\left(\epsilon_{i}\right) \frac{1}{\lambda} E(X \mid \delta=1)+\frac{1}{2 \lambda^{2}} \epsilon_{i} E(X \mid \delta=1)\right\}
\end{aligned}
$$

which simplifies to

$$
\begin{aligned}
b\left(\delta_{i}, X_{i}, \epsilon_{i}\right)= & E\left[\delta \left\{X X^{\top}-\frac{1}{2} X E(X \mid \delta=1)^{\top}-\frac{1}{2} E(X \mid \delta=1) X^{\top}\right.\right. \\
& \left.\left.+\frac{1}{2} E(X \mid \delta=1) E(X \mid \delta=1)^{\top}\right\}\right]^{-1} \\
& \delta_{i}\left[\operatorname{sign}\left(\epsilon_{i}\right) \lambda\{X-E(X \mid \delta=1)\}+\frac{1}{2} \epsilon_{i} E(X \mid \delta=1)\right] .
\end{aligned}
$$


The final form is found by factoring,

$$
\begin{aligned}
= & E\left[\delta\left\{X-\frac{1}{2} E(X \mid \delta=1)\right\}\left\{X-\frac{1}{2} E(X \mid \delta=1)\right\}^{\top}\right. \\
& \left.\left.+\delta \frac{1}{4} E(X \mid \delta=1) E(X \mid \delta=1)^{\top}\right\}\right]^{-1} \\
& \delta_{i}\left[\operatorname{sign}\left(\epsilon_{i}\right) \lambda\{X-E(X \mid \delta=1)\}+\frac{1}{2} \epsilon_{i} E(X \mid \delta=1)\right] .
\end{aligned}
$$

By the Hajek-Le Cam Theory the OLS estimator will have a random term which introduces more variability than an efficient estimator. This random term is

$$
\begin{aligned}
& \frac{1}{\sqrt{n}} \sum_{i=1}^{n}\left(E\left[\delta X X^{\top}\right]^{-1} \delta_{i} X_{i} \epsilon_{i}-E\left[\delta(X-E[X \mid \delta=1])(X-E[X \mid \delta=1])^{\top}\right.\right. \\
& \left.+\frac{1}{2} \delta E[X \mid \delta=1] E[X \mid \delta=1]^{\top}\right]^{-1} \\
& \left.\left\{\operatorname{sign}\left(\epsilon_{i}\right) \delta_{i} \lambda\left(X_{i}-E[X \mid \delta=1]\right)+\frac{1}{2} \delta_{i} \epsilon_{i} E[X \mid \delta=1]\right\}\right) .
\end{aligned}
$$

By showing that for a special case scenario this component is not zero, we will show the OLS is not always an efficient estimator. Consider conditionally centered $X$ 's where $E[X \delta]=0$. This is equivalent to $E[X \mid \delta=1]=0$, and Equation 2.8 becomes

$$
\begin{aligned}
& \frac{1}{\sqrt{n}} \sum_{i=1}^{n}\left(E\left[\delta X X^{\top}\right]^{-1} \delta_{i} X_{i} \epsilon_{i}-E\left[\delta X X^{\top}\right]^{-1} \operatorname{sign}\left(\epsilon_{i}\right) \delta_{i} \lambda X_{i}\right) \\
= & \frac{1}{\sqrt{n}} E\left[\delta X X^{\top}\right]^{-1} \sum_{i=1}^{n}\left(\delta_{i} X_{i}\left(\epsilon_{i}-\operatorname{sign}\left(\epsilon_{i}\right) \lambda\right)\right) \\
= & \frac{1}{\sqrt{n}} E\left[\delta X X^{\top}\right]^{-1} \sum_{i=1}^{n}\left(\delta_{i} X_{i}\left(\epsilon_{i}-\lambda 1_{\epsilon_{i}>0}+\lambda 1_{\epsilon_{i}<0}\right)\right) .
\end{aligned}
$$

This does not equal zero, and so the OLS estimator is not efficient for this model. The following Lemma compares the MSE for the efficient estimator with the MSE for the OLS estimator. The MSE for for a random square integrable estimator $\hat{\vartheta}$ is 
defined as

$$
\operatorname{MSE}(\hat{\vartheta})=E\left[(\hat{\vartheta}-E(\hat{\vartheta}))(\hat{\vartheta}-E(\hat{\vartheta}))^{\top}\right]
$$

Lemma II.6 Under the model where the error term is in fact double exponential and where $E(X \delta)=0$ the ratio of the MSE for the OLS estimator versus the efficient estimator is $M S E\left(\hat{\vartheta}_{E F F}\right)=\frac{1}{2} M S E\left(\hat{\vartheta}_{O L S}\right)$.

Proof: First to find the MSE of the OLS estimator using Equation 2.7.

$$
\begin{aligned}
n M S E\left(\hat{\vartheta}_{O L S}\right) & =E\left[\left(\hat{\vartheta}_{O L S}-\vartheta\right)\left(\hat{\vartheta}_{O L S}-\vartheta\right)^{\top}\right] \\
& \rightarrow E\left[\left(E\left[\delta X X^{\top}\right]^{-1} \delta X \epsilon\right)\left(E\left[\delta X X^{\top}\right]^{-1} \delta X \epsilon\right)^{\top}\right] \\
& =E\left[E\left[\delta X X^{\top}\right]^{-1} \delta X \epsilon \epsilon X^{\top} \delta E\left[\delta X X^{\top}\right]^{-1}\right] \\
& =E\left[\epsilon^{2} E\left[\delta X X^{\top}\right]^{-1} \delta X X^{\top} E\left[\delta X X^{\top}\right]^{-1}\right] \\
& =\sigma^{2} E\left[\delta X X^{\top}\right]^{-1}=2 \lambda^{2} E\left[\delta X X^{\top}\right]^{-1} .
\end{aligned}
$$

Now to find the MSE for the efficient estimator using the influence function,

$$
\begin{aligned}
n M S E\left(\hat{\vartheta}_{E F F}\right) & =E\left[\left(\hat{\vartheta}_{E F F}-\vartheta\right)\left(\hat{\vartheta}_{E F F}-\vartheta\right)^{\top}\right] \\
& \rightarrow E\left[\left(E\left[\delta X X^{\top}\right]^{-1} \operatorname{sign}(\epsilon) \delta \lambda X\right)\left(E\left[\delta X X^{\top}\right]^{-1} \operatorname{sign}(\epsilon) \delta \lambda X\right)^{\top}\right] \\
& =E\left[E\left[\delta X X^{\top}\right]^{-1} \operatorname{sign}(\epsilon) \delta \lambda X X^{\top} \lambda \delta \operatorname{sign}(\epsilon) E\left[\delta X X^{\top}\right]^{-1}\right] \\
& =E\left[\lambda^{2} E\left[\delta X X^{\top}\right]^{-1} \delta X X^{\top} E\left[\delta X X^{\top}\right]^{-1}\right] \\
& =\lambda^{2} E\left[\delta X X^{\top}\right]^{-1} .
\end{aligned}
$$

Then the ratio for the MSE for each estimator is

$$
\operatorname{MSE}\left(\hat{\vartheta}_{O L S}\right)^{-1} M S E\left(\hat{\vartheta}_{E F F}\right)=\frac{\lambda^{2} E\left[\delta X X^{\top}\right]^{-1} E[\delta X X]}{2 \lambda^{2}}=\frac{1}{2} .
$$


This shows that asymptotically the efficient estimator will have half the variance of the OLS estimator.

2. One step improvement estimator

One estimator which is asymptotically efficient for linear regression without missing responses is the One Step Improvement estimator (OSI) described in Forrester et al. (2003). This estimator can be modified analogously for the missing data situation as shown below.

This requires an initial estimate of $\vartheta$, call it $\bar{\vartheta}$, which must be $\sqrt{n}$ consistent and discretized. The Ordinary Least Squares estimator, even if the error distribution is not normal is often used in practice as this original estimate. This estimator is then "improved" by using a Newton-Raphson method with a direct estimator of the influence function. Define

$$
\mu=E(X \mid \delta=1) \quad \sigma^{2}=E\left(\epsilon^{2}\right) .
$$

We can estimate $\mu$ and $\sigma^{2}$ with $\hat{\mu}$ and $\hat{\sigma^{2}}$ where

$$
\begin{aligned}
\hat{\mu} & =\frac{\sum_{i=1}^{n} \delta_{i} X_{i}}{\sum_{i=1}^{n} \delta_{i}} \\
\hat{\sigma^{2}} \bar{\vartheta} & =\frac{\sum_{i=1}^{n} \delta_{i} \epsilon_{i}(\bar{\vartheta})^{2}}{\sum_{i=1}^{n} \delta_{i}} \\
\epsilon(\vartheta) & =Y-\vartheta^{\top} X \\
\hat{\zeta}_{\bar{\vartheta}}(X, Y, \delta) & =[X-\hat{\mu}] \hat{l}\{\epsilon(\bar{\vartheta})\}+\hat{\mu} \epsilon(\bar{\vartheta}) / \sigma_{\bar{\vartheta}}^{2} .
\end{aligned}
$$

Then the One Step Improvement estimator is

$$
\vartheta_{O S I}=\bar{\vartheta}+\left\{\sum_{i=1}^{n} \delta_{i} \hat{\zeta}_{\bar{\vartheta}}\left(X_{i}, Y_{i}, \delta_{i}\right) \hat{\zeta}_{\bar{\vartheta}}\left(X_{i}, Y_{i}, \delta_{i}\right)^{\top}\right\}^{-1} \sum_{i=1}^{n} \delta_{i} \hat{\zeta}_{\bar{\vartheta}}\left(X_{i}, Y_{i}, \delta_{i}\right)
$$


where

$$
\hat{l}_{\bar{\vartheta}}(\epsilon)=\frac{-\widehat{\hat{f}}_{n}(\epsilon)}{\hat{f}_{n}(\epsilon)}
$$

for a kernel based estimate of the error density $\hat{f}_{n}(\epsilon)$. Call the density estimate $\hat{f}_{n}^{\prime}(\epsilon)$. The derivative is estimated as shown in Zhi (2012) using the kernel estimate $K$ with bandwidth $h$ by

$$
\hat{f}_{n}^{\prime}(\epsilon)=\frac{1}{n h^{2}} \sum_{i=1}^{n} K^{\prime}\left(\frac{x-x_{i}}{h}\right) .
$$

The OSI estimator is efficient because it uses all the model information, including the independence between $\epsilon$ and $X$.

\section{Maximum empirical likelihood estimator}

An alternative efficient estimator is the Maximum Empirical Likelihood estimator (MELE) which is explained by Peng and Schick (2012). This method maximizes the empirical likelihood $R_{n}(\vartheta)$ with respect to $\vartheta$. The MELE estimate is shown to be efficient and equivalent to the OSI estimator in the model with complete observations by Owen (1988). In the case of missing responses the estimator uses the subset of responses which were observed, which is the same subset used for the OSI estimator. Thus, following the reasoning used by Koul et al. (2012), the efficiency is preserved in the MELE estimator. This approach is based on estimating the likelihood in $L_{2,0}$. The empirical likelihood is

$$
\begin{gathered}
R_{n}(\vartheta)=\sup \left\{\prod_{i=1}^{n} n \pi_{i}: \pi_{i} \in[0,1], \sum_{i=1}^{n} \pi_{i}=1, \sum_{i=1}^{n} \pi_{i} \delta_{i} \epsilon_{i}(\vartheta)=0,\right. \\
\left.\sum_{i=1}^{n} \pi_{i} \delta_{i} c_{i k}(\vartheta)=0, k=1, \cdots, m\right\} .
\end{gathered}
$$

The constraint $\sum_{i=1}^{n} \pi_{i} \delta_{i} \epsilon_{i}(\vartheta)=\sum_{i=1}^{n} \pi_{i} \delta_{i}\left(Y_{i}-\vartheta^{\top} X\right)=0$ comes from the assump- 
tion that the errors have mean zero. The $m$ constraints involving $c_{i k}$ 's refer to the independence assumption between $X$ and $\epsilon$. The idea is as follows: independence between $X$ and $\epsilon$ implies $E\{(X-E X) a(\epsilon)\}=0$ for any function $a \in L_{2,0}(F)$, where $F$ is the distribution of $\epsilon$. If $F$ is continuous, then $F(\epsilon)$ is uniformly distributed on the interval $(0,1)$. An orthonormal basis of $L_{2,0}(F)$ is $\phi_{1} \circ F, \phi_{2} \circ F, \cdots$ where the $\phi_{k}$ denote a basis of $L_{2}(U)$. Define $\mathbb{F}_{\vartheta}$ as the residual based empirical distribution function for $F$. Then the estimated constraints are

$$
c_{i k}(\vartheta)=\left(X_{i}-\hat{\mu}\right) \phi_{k}\left[\mathbb{F}_{\vartheta}\left\{\epsilon_{i}(\vartheta)\right\}\right], k=1,2, \cdots, m
$$

for some integer $m=m_{n} \rightarrow \infty$ as $n \rightarrow \infty$. As shown in Owen (2001) this maximization problem will have exactly one solution in the simple linear case with probability tending to one. For more detail and an implementation of the code in $R$ see the function el.test in the $R$ package emplik. This estimate incorporates all information in the model rendering it efficient for $\vartheta$.

C. Simulation results for estimating the parameter

To simulate these results let $\vartheta=3, E[\epsilon]=0, X \sim \operatorname{Uniform}(0,2)$, with $\epsilon$ and $X$ independent. Then $\hat{\vartheta}$ was calculated using each of the following methods:

1. OLS: Ordinary Least Squares. Estimator will be efficient when the unknown error is in fact normally distributed.

2. OSI: One Step Improvement. Estimator is asymptotically efficient.

3. MELE1: Maximum Empirical Likelihood Estimator with one constraint. For small sample sizes one constraint could be sufficient to achieve efficiency. 
4. MELE2: Maximum Empirical Likelihood Estimator with two constraints on the basis. The extra constraint handles larger sample sizes.

5. MELE3: Maximum Empirical Likelihood Estimator with three constraints on the basis. The larger the sample size the more constraints needed to achieve efficiency.

For each method of estimation the data is used only where the response is observed. Thus in this section, we will work under the assumption of no missing data, meaning $\delta_{i}=1$ for all $i$.

The Mean Square Error (MSE) was calculated for each simulation using various methods of estimating $\vartheta$. The methods include Ordinary Least Squares (OLS), One Step Improvement (OSI), and Maximum Empirical Likelihood with one, two, or three constraints on the basis (MELE1, MELE2 and MELE3 respectively).

The efficiency discussed earlier was verified for many error distributions. When the errors are normal all the methods were practically indistinguishable, as expected. Two interesting scenarios are shown. Figure 1 has $t_{2}$ errors, and it can be seen that the OLS estimator has a larger MSE, while the other methods are clear improvements. The OSI is worse for small sample sizes because of the difficulty of estimating the score function. Figure 2 has gamma errors shifted to have a mean of zero. The OLS again has more variance, and now the effect of extra constraints in the MELE basis can be seen since MELE3 has the smallest MSE.

For other distributions including the normal which are not shown here see Appendix A, 


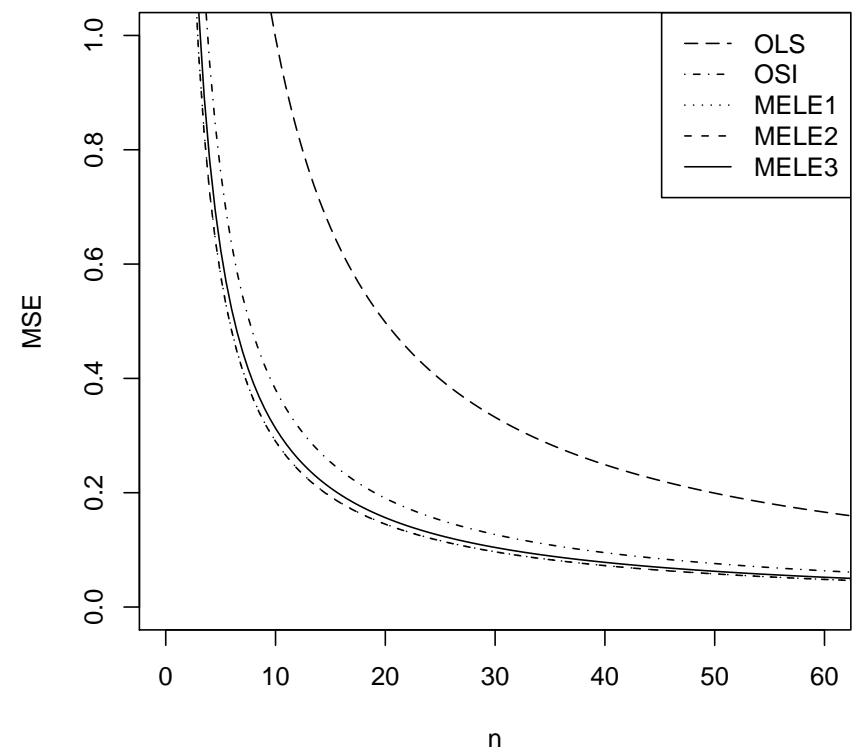

Fig. 1. MSE for various methods of estimating $\vartheta$ under $t_{2}$ errors.

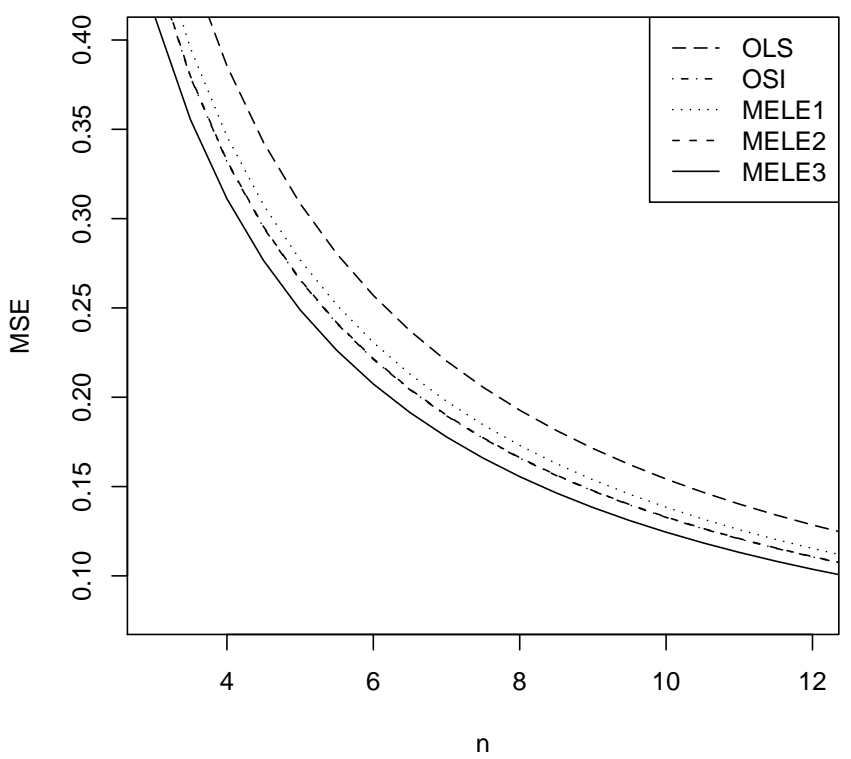

Fig. 2. MSE for various methods of estimating $\vartheta$ under gamma errors. 


\section{CHAPTER III}

\section{ESTIMATING THE MEAN RESPONSE IN REGRESSION}

In this section the goal is to estimate $E[Y]$. Define $\widehat{E(Y)}$ as the estimate for an estimator of $E(Y)$. Various estimators will be compared using the asymptotic variance

$$
A V=\lim _{n \rightarrow \infty} E\left(n[\widehat{E(Y)}-E\{\widehat{E(Y)}\}]^{2}\right)
$$

The methods that will compared are Listwise Deletion, a Propensity Score method, Partial Imputation, and Full Imputation.

\section{A. Listwise deletion}

Listwise Deletion does not use any form of imputation. Any data entry where the response (or the covariate) is missing is simply deleted from the dataset. This is the default method of handling missing data for certain programs. The estimator is

$$
\widehat{E(Y)}_{L D}=\frac{\sum_{i=1}^{n} \delta_{i} Y_{i}}{\sum_{i=1}^{n} \delta_{i}}
$$

This estimator is not consistent. This can be seen from

$$
\begin{aligned}
E\left\{\widehat{\left.E(Y)_{L D}\right\}}\right. & =E\left(\frac{\sum_{i=1}^{n} \delta_{i} Y_{i}}{\sum_{i=1}^{n} \delta_{i}}\right) \\
& =E\left(\frac{\frac{1}{n} \sum_{i=1}^{n} \delta_{i} Y_{i}}{\frac{1}{n} \sum_{i=1}^{n} \delta_{i}}\right) \\
& \rightarrow E\left\{\frac{E(\delta Y)}{E(\delta)}\right\} \\
& =E(Y \mid \delta=1) .
\end{aligned}
$$


The asymptotic result comes from the Law of Large Numbers. This expectation should make sense, since it is the mean of the response where the data is not missing, but this is not in general unbiased for $E[Y]$. There are cases where listwise is unbiased, for example, when the missing structure is symmetric across a symmetric covariate $X$. Theorem III.1 explains the implication of this assumption.

Theorem III.1 When the missing structure is symmetric over symmetric covariates

$$
E(\delta X)=E(\delta) E(X)
$$

Proof: For simplicity and reasons of clarity we assume the density of $X$, namely $g(x)$, is square integrable. Further define the density of $X$ given $\delta=1$ as $g_{1}(x)$ and assume it is square integrable. The assumption that the missing structure is symmetric over the symmetric covariate means

$$
\left.g_{1}\{E(X)-x)\right\}=g_{1}\{E(X)+x\} .
$$

Then because $g_{1}(x)$ is a density,

$$
\int_{0}^{\infty} g_{1}\{E(X)+x\} d x+\int_{0}^{\infty} g_{1}\{E(X)-x\} d x=1,
$$

which implies

$$
\int_{0}^{\infty} g_{1}\{E(X)+x\} d x=1 / 2
$$


Because $\delta$ is either zero or one, the following holds,

$$
\begin{aligned}
E(\delta X) & =0 E(X \mid \delta=0)\{1-E(\delta)\}+1 E(X \mid \delta=0) E(\delta) \\
& =E(X \mid \delta=1) E(\delta) \\
& =E(\delta) \int_{-\infty}^{\infty} x g_{1}(x) d x \\
& =E(\delta)\left\{\int_{-\infty}^{E(X)} x g_{1}(x) d x+\int_{E(X)}^{\infty} x g_{1}(x) d x\right\} .
\end{aligned}
$$

Now changing the indexes so the integrals sum over the same area, but start from $E(X)$ and then $x$ moves toward the limit,

$$
\begin{aligned}
E(\delta X)= & E(\delta)\left[\int_{0}^{\infty}\{E(X)-x\} g_{1}\{E(X)-x\} d x\right. \\
& \left.+\int_{0}^{\infty}\{E(X)+x\} g_{1}\{E(X)+x\} d x\right]
\end{aligned}
$$

Using the symmetry of the missing structure from Equation 3.2 ,

$$
\begin{aligned}
E(\delta X)= & E(\delta)\left[\int_{0}^{\infty}\{E(X)-x\} g_{1}\{E(X)+x\} d x\right. \\
& \left.+\int_{0}^{\infty}\{E(X)+x\} g_{1}\{E(X)+x\} d x\right] \\
= & E(\delta)\left(\int_{0}^{\infty}[\{E(X)-x\}+\{E(X)+x\}] g_{1}\{E(X)+x\} d x\right) \\
= & E(\delta) \int_{0}^{\infty} 2 E(X) g_{1}\{E(X)+x\} d x \\
= & E(\delta) 2 E(X) \int_{0}^{\infty} g_{1}\{E(X)+x\} d x .
\end{aligned}
$$

By Equation 3.3

$$
\begin{aligned}
E(\delta X) & =E(\delta) 2 E(X) 1 / 2 \\
& =E(\delta) E(X) .
\end{aligned}
$$


Using this theorem for Listwise Deletion when the missing structure is symmetric over the covariate then

$$
E(X \mid \delta=1)=E(X) \Longleftrightarrow E(\delta X)=E(1 * X \mid \delta=1) E(\delta)=E(\delta) E(X)
$$

This means for linear regression if the data is missing equally on both sides of $E(X)$ then the estimate of the mean without the missing data will be asymptotically consistent.

The asymptotic variance for Listwise Deletion using Equation 3.1 is

$$
\begin{aligned}
A V_{L D} & =\lim _{n \rightarrow \infty} E\left[n\left\{\frac{\sum_{i=1}^{n} \delta_{i} Y_{i}}{\sum_{i=1}^{n} \delta_{i}}-\frac{E(\delta Y)}{E(\delta)}\right\}^{2}\right] \\
& =\lim _{n \rightarrow \infty} n E\left\{\frac{\sum_{i=1}^{n} \sum_{j=1}^{n} \delta_{i} \delta_{j} Y_{i} Y_{j}}{\sum_{i=1}^{n} \delta_{i} \sum_{j=1}^{n} \delta_{j}}-2 \frac{E(\delta Y)}{E(\delta)} \frac{\sum_{i=1}^{n} \delta_{i} Y_{i}}{\sum_{i=1}^{n} \delta_{i}}+\frac{E^{2}(\delta Y)}{E^{2}(\delta)}\right\} .
\end{aligned}
$$

By separating the sums,

$$
\begin{aligned}
A V_{L D}= & \lim _{n \rightarrow \infty} n E\left\{\frac{\sum_{i=1}^{n} \delta_{i} Y_{i}^{2}+\sum_{i=1}^{n} \delta_{i} Y_{i} \sum_{j=1, j \neq i}^{n} \delta_{j} Y_{j}}{\sum_{i=1}^{n} \delta_{i}+\sum_{i=1}^{n} \delta_{i} \sum_{j=1, j \neq i}^{n} \delta_{j}}\right. \\
& \left.-2 \frac{E(\delta Y)}{E(\delta)} \frac{\sum_{i=1}^{n} \delta_{i} Y_{i}}{\sum_{i=1}^{n} \delta_{i}}+\frac{E^{2}(\delta Y)}{E^{2}(\delta)}\right\}
\end{aligned}
$$

Including the proper constant of $n$ ensures that each summation is an average, which renders the equation in the form

$$
\begin{aligned}
A V_{L D}= & \lim _{n \rightarrow \infty} n E\left\{\frac{\frac{1}{n} \frac{1}{n} \sum_{i=1}^{n} \delta_{i} Y_{i}^{2}+\frac{n-1}{n} \frac{1}{n} \sum_{i=1}^{n} \delta_{i} Y_{i} \frac{1}{n-1} \sum_{j=1, j \neq i}^{n} \delta_{j} Y_{j}}{\frac{1}{n} \frac{1}{n} \sum_{i=1}^{n} \delta_{i}+\frac{n-1}{n} \frac{1}{n} \sum_{i=1}^{n} \delta_{i} \frac{1}{n-1} \sum_{j=1, j \neq i}^{n} \delta_{j}}\right. \\
& \left.-2 \frac{E(\delta Y)}{E(\delta)} \frac{\frac{1}{n} \sum_{i=1}^{n} \delta_{i} Y_{i}}{\frac{1}{n} \sum_{i=1}^{n} \delta_{i}}+\frac{E^{2}(\delta Y)}{E^{2}(\delta)}\right\} .
\end{aligned}
$$

By the law of large numbers each average converges to its finite expected value. By Slutsky's theorem the summations in the equation can be evaluated separately. The 
equation then simplifies to

$$
\begin{aligned}
A V_{L D} & =\lim _{n \rightarrow \infty} n\left\{\frac{\frac{1}{n} E\left(\delta Y^{2}\right)+\frac{n-1}{n} E^{2}(\delta Y)}{E^{2}(\delta)}-2 \frac{E^{2}(\delta Y)}{E^{2}(\delta)}+\frac{E^{2}(\delta Y)}{E^{2}(\delta)}\right\} \\
& =\lim _{n \rightarrow \infty} n\left\{\frac{1}{n} \frac{E\left(\delta Y^{2}\right)}{E^{2}(\delta)}+\frac{n-1}{n} \frac{E^{2}(\delta Y)}{E^{2}(\delta)}-\frac{E^{2}(\delta Y)}{E^{2}(\delta)}\right\} \\
& =\frac{E\left(\delta Y^{2}\right)}{E^{2}(\delta)}-\frac{E^{2}(\delta Y)}{E^{2}(\delta)} .
\end{aligned}
$$

Thus the listwise deletion method is biased unless the missing structure is symmetric across $X$ as shown in Theorem III.1, in which case the asymptotic variance would be

$$
A V_{L D}=\frac{\vartheta^{\top} E\left(\delta X X^{\top}\right) \vartheta}{E^{2}(\delta)}+\frac{\sigma^{2}}{E(\delta)}-\vartheta^{\top} E(X) E(X)^{\top} \vartheta
$$

This variance will be compared with other methods in Chapter V.

\section{B. Propensity score}

Another method which does not use imputation but is unbiased is the propensity score method proposed by Rosenbaum and Rubin (1983). For this type of no imputation the data where the response is not observed are deleted from the dataset, but responses which are observed are weighted with the probability of being observed. The estimate for the mean response is

$$
\widehat{E(Y)}_{P S}=\frac{1}{n} \sum_{i=1}^{n} \frac{\delta_{i} Y_{i}}{E\left(\delta \mid X_{i}\right)}
$$

If $E\left(\delta \mid X_{i}\right)$ is not known then it can be estimated empirically as done by Dong and Song (2009). It will be assumed that $E(\delta \mid X)$ is bounded away from zero on the support of $X$. Note this equation does not require an estimate of $\vartheta$. The estimator 
is consistent under the Missing At Random assumption since

$$
\begin{aligned}
E\left\{\frac{1}{n} \sum_{i=1}^{n} \frac{\delta_{i} Y_{i}}{E\left(\delta \mid X_{i}\right)}\right\} & =E\left\{\frac{\delta Y}{E(\delta \mid X)}\right\} \\
& =E\left[E\left\{\frac{\delta Y}{E(\delta \mid X)} \mid X\right\}\right] \\
& =E\left\{\frac{E(\delta \mid X) E(Y \mid X)}{E(\delta \mid X)}\right\} \\
& =E(Y) .
\end{aligned}
$$

Deleting the responses which are not observed is a loss of information, and as such the propensity score method is in general not efficient. For a discussion on the disadvantages of such methods see Bell et al. (2009) for details on the bias and variance of such methods.

The asymptotic variance for the propensity score method using Equation 3.6 is

$$
\begin{aligned}
A V_{P S} & =\lim _{n \rightarrow \infty} n E\left[\left\{\frac{1}{n} \sum_{i=1}^{n} \frac{\delta_{i} Y_{i}}{E\left(\delta \mid X_{i}\right)}-E(Y)\right\}^{2}\right] \\
& =\lim _{n \rightarrow \infty} n E\left\{\frac{1}{n^{2}} \sum_{i=1}^{n} \sum_{j=1}^{n} \frac{\delta_{i} \delta_{j} Y_{i} Y_{j}}{E\left(\delta \mid X_{i}\right) E\left(\delta \mid X_{j}\right)}-2 E(Y) \frac{1}{n} \sum_{i=1}^{n} \frac{\delta_{i} Y_{i}}{E\left(\delta \mid X_{i}\right)}+E^{2}(Y)\right\} \\
& =\lim _{n \rightarrow \infty}\left[\frac{1}{n} \sum_{i=1}^{n} \sum_{j=1}^{n} E\left\{\frac{\delta_{i} \delta_{j} Y_{i} Y_{j}}{E\left(\delta \mid X_{i}\right) E\left(\delta \mid X_{j}\right)}\right\}-2 n E(Y) E\left\{\frac{\delta Y}{E(\delta \mid X)}\right\}+n E^{2}(Y)\right] .
\end{aligned}
$$


By separating the sums

$$
\begin{aligned}
A V_{P S}= & \lim _{n \rightarrow \infty}\left[\frac{1}{n} \sum_{i=1}^{n} E\left\{\frac{\delta_{i}^{2} Y_{i}^{2}}{E^{2}\left(\delta \mid X_{i}\right)}\right\}+\frac{1}{n} \sum_{i=1}^{n} \sum_{j=1, j \neq i}^{n} E\left\{\frac{\delta_{i} Y_{i} \delta_{j} Y_{j}}{E\left(\delta \mid X_{i}\right) E\left(\delta \mid X_{j}\right)}\right\}\right. \\
& \left.-2 n E(Y) E\left\{\frac{E(\delta \mid X) E(Y \mid X)}{E(\delta \mid X)}\right\}+n E^{2}(Y)\right] \\
= & \lim _{n \rightarrow \infty}\left[E\left\{\frac{\delta Y^{2}}{E^{2}(\delta \mid X)}\right\}\right. \\
& +(n-1) \sum_{i=1}^{n} \sum_{j=1, j \neq i}^{n} E\left\{\frac{E\left(\delta \mid X_{i}\right) E\left(Y \mid X_{i}\right) E\left(\delta \mid X_{j}\right) E\left(Y \mid X_{j}\right)}{E\left(\delta \mid X_{i}\right) E\left(\delta \mid X_{j}\right)}\right\} \\
& \left.-2 n E^{2}(Y)+n E^{2}(Y)\right] \\
= & \lim _{n \rightarrow \infty}\left[E\left\{\frac{E(\delta \mid X) E\left(Y^{2} \mid X\right)}{E^{2}(\delta \mid X)}\right\}+(n-1) E\left\{E\left(Y \mid X_{i}\right) E\left(Y \mid X_{j}\right)\right\}-n E^{2}(Y)\right] \\
= & \lim _{n \rightarrow \infty}\left[E\left\{\frac{E\left(Y^{2} \mid X\right)}{E(\delta \mid X)}\right\}+(n-1) E^{2}(Y)-n E^{2}(Y)\right] \\
= & E\left\{\frac{E\left(Y^{2} \mid X\right)}{E(\delta \mid X)}\right\}-E^{2}(Y) .
\end{aligned}
$$

Now using $Y=\vartheta^{\top} X+\epsilon$,

$$
A V_{P S}=E\left\{\frac{\vartheta^{\top} X X^{\top} \vartheta+\sigma^{2}}{E(\delta \mid X)}\right\}-\vartheta^{\top} E(X) E(X)^{\top} \vartheta
$$

This variance will be compared with other methods in Chapter V.

\section{Partial imputation}

In partial imputation only the missing responses are imputed. The estimate is

$$
\widehat{E(Y)_{P I}}=\frac{1}{n} \sum_{i=1}^{n}\left(\delta_{i} Y_{i}+\left(1-\delta_{i}\right) \hat{\vartheta}^{\top} X_{i}\right)
$$

This method requires an estimate of $\vartheta$. The asymptotic variance of partial imputation depends in part on the variability of $\hat{\vartheta}$. When $Y$ is observed and used instead of 
$\hat{\vartheta} X$ then information about the regression structure is lost. This implies partial imputation is not in general efficient.

The efficiency of the estimator used for $\vartheta$ will affect the asymptotic variance of the partially imputed estimator. This can be seen by examining the influence function of $\hat{\vartheta}, b(\delta, X, \epsilon)$. Assume $\hat{\vartheta}$ is a $\sqrt{n}$ consistent estimator of $\vartheta$. Then the estimate of $\vartheta$ can be expanded in asymptotically linear form using Equation 2.1 to

$$
\sqrt{n}(\hat{\vartheta}-\vartheta)=\frac{1}{\sqrt{n}} \sum_{i=1}^{n} b\left(\delta_{i}, X_{i}, \epsilon_{i}\right)+o_{p}(1)
$$

where $E\left\{b\left(\delta_{i} . X_{i}, \epsilon_{i}\right)\right\}=0$.

To simplify the notation the influence function will be written as $b_{i}$ rather than $b\left(\delta_{i}, X_{i}, \epsilon_{i}\right)$. To simplify the equations the notation $\doteq$ will be used to denote equals on the order of $n^{-1 / 2}$. In other words, $\doteq$ allows suppression of the term $o\left(n^{-1 / 2}\right)$. For more detail on the implications of $\sqrt{n}$ estimation in a semiparametric model refer to Schick (1996a), or for a partly linear model refer to Schick (1996b). Then the estimator $\hat{\vartheta}$ can be written as

$$
\hat{\vartheta}=\vartheta+\frac{1}{n} \sum_{i=1}^{n} b_{i}+o_{p}\left(n^{-1 / 2}\right) .
$$

Also throughout the following proofs the subscripts $X_{i}$ vs. $X_{j}$ will emphasize when two variables are independent of each other. The next theorem shows the partially imputed estimator is asymptotically unbiased.

Theorem III.2 The expected value for partial imputation is

$$
E\left\{\widehat{E(Y)_{P I}}\right\} \doteq E(Y) .
$$


PRoOF: The following expected value will be used frequently:

$$
\begin{aligned}
E\left(\hat{\vartheta}^{\top} X\right) & \doteq E\left\{\left(\vartheta+\frac{1}{n} \sum_{i=1}^{n} b_{i}\right)^{\top} X_{j}\right\} \\
& \doteq \vartheta^{\top} E(X)+\frac{n-1}{n} E\left(b_{i}^{\top} X_{j}\right)+\frac{1}{n} E\left(b^{\top} X\right) \\
& \doteq \vartheta^{\top} E(X)+\frac{1}{n} E\left(b^{\top} X\right) .
\end{aligned}
$$

By similar proof it can be shown that

$$
\begin{aligned}
E\left(\delta \hat{\vartheta}^{\top} X\right) & \doteq \vartheta^{\top} E(\delta X)+\frac{1}{n} E\left(\delta b^{\top} X\right) \\
E\left(\hat{\vartheta}^{\top} X X^{\top}\right) & \doteq \vartheta^{\top} E\left(X X^{\top}\right)+\frac{1}{n} E\left(b^{\top} X X^{\top}\right) .
\end{aligned}
$$

Using these expected values the expected value for partial imputation can be found as

$$
\begin{aligned}
E\left\{\widehat{E(Y)_{P I}}\right\} & =E\left\{\frac{1}{n} \sum_{i=1}^{n} \delta_{i} Y_{i}+\left(1-\delta_{i}\right) \hat{\vartheta} X_{i}^{\top}\right\} \\
& =E(\delta Y)+E\left(\hat{\vartheta}^{\top} X\right)-E\left(\delta \hat{\vartheta}^{\top} X\right) .
\end{aligned}
$$

Using Equation 3.9 ,

$$
\begin{aligned}
E\left\{\widehat{E(Y)_{P I}}\right\} & \doteq E\left\{\delta\left(\vartheta^{\top} X+\epsilon\right)\right\}+\vartheta^{\top} E(X)+\frac{1}{n} E\left(b^{\top} X\right)-\vartheta^{\top} E(\delta X)-\frac{1}{n} E\left(\delta b^{\top} X\right) \\
& \doteq \vartheta^{\top} E(\delta X)+\vartheta^{\top} E(X)-\vartheta^{\top} E(\delta X)+\frac{1}{n} E\left(b^{\top} X\right)-\frac{1}{n} E\left(\delta b^{\top} X\right) .
\end{aligned}
$$

Now using the fact that these results are of order $o_{p}\left(n^{-1 / 2}\right)$,

$$
\begin{aligned}
E\left\{\widehat{E(Y)_{P I}}\right\} & \doteq \vartheta^{\top} E(X) \\
& \doteq E\left(\vartheta^{\top} X+\epsilon\right) \\
& \doteq E(Y) .
\end{aligned}
$$

The next theorem will identify the asymptotic variance. 
Theorem III.3 The asymptotic variance for partial imputation is

$$
\begin{aligned}
A V_{P I}= & \vartheta^{\top}\left\{E\left(X X^{\top}\right)-E(X) E(X)^{\top}\right\} \vartheta+\sigma^{2} E(\delta) \\
& +2 E(b \delta \epsilon)^{\top}\{E(X)-E(\delta X)\}-2 \vartheta^{\top} E(\delta X) E\left(\delta X^{\top} b\right) \\
& -2 \vartheta^{\top} E\left(\delta X b^{\top}\right)\{E(X)-E(\delta X)\}+2 \vartheta^{\top} E\left(X b^{\top}\right) E(X) \\
& +\{E(X)-E(\delta X)\}^{\top} E\left(b b^{\top}\right)\{E(X)-E(\delta X)\} .
\end{aligned}
$$

Proof: The following expected value will be useful later. The subscripts on $X$ help indicate independent instances of the same random variable.

$$
\begin{aligned}
E\left(\hat{\vartheta}^{\top} X_{i} X_{j}^{\top}\right) \doteq & E\left\{\left(\vartheta^{\top}+\frac{1}{n} \sum_{i=1}^{n} b_{i}^{\top}\right) X_{j} X_{k}^{\top}\right\} \\
\doteq & E\left(\vartheta^{\top} X_{i} X_{j}^{\top}+\frac{1}{n} b_{i}^{\top} X_{i} X_{j}^{\top}+\frac{1}{n} b_{i}^{\top} X_{j} X_{i}^{\top}+\frac{1}{n} \sum_{i \neq \text { nor j }}^{\text {n-2 terms }} b_{i}^{\top} X_{j} X_{k}^{\top}\right) \\
\doteq & \vartheta^{\top} E(X) E(X)^{\top}+\frac{1}{n} E\left(b^{\top} X\right) E(X)^{\top}+\frac{1}{n} E(X)^{\top} E\left(b X^{\top}\right) \\
& +\frac{n-2}{n} E(b)^{\top} E(X) E(X)^{\top} .
\end{aligned}
$$

Using the fact that $E(b)=0$,

$$
E\left(\hat{\vartheta}^{\top} X_{i} X_{j}^{\top}\right) \doteq \vartheta^{\top} E(X) E(X)^{\top}+\frac{1}{n}\left\{E\left(b^{\top} X\right) E(X)^{\top}+E(X)^{\top} E\left(b X^{\top}\right)\right\}(3.1
$$

By similar proof

$$
\begin{aligned}
E\left(\delta_{j} \hat{\vartheta}^{\top} X_{i} X_{j}^{\top}\right) & \doteq \vartheta^{\top} E(\delta X) E(X)^{\top}+\frac{1}{n}\left\{E\left(b^{\top} X\right) E(\delta X)^{\top}+E(X)^{\top} E\left(\delta b X^{\top}\right)\right\} \\
E\left(\delta_{i} \delta_{j} \hat{\vartheta}^{\top} X_{i} X_{j}^{\top}\right) & \doteq \vartheta^{\top} E(\delta X) E(\delta X)^{\top}+\frac{1}{n}\left\{E\left(\delta b^{\top} X\right) E(\delta X)^{\top}+E(\delta X)^{\top} E\left(\delta b X^{\top}\right)\right\} .
\end{aligned}
$$


Now an expected value involving $\hat{\vartheta}$ and $\epsilon$,

$$
\begin{aligned}
E\left(\epsilon_{i} \hat{\vartheta}^{\top} X_{j}\right) & \doteq E\left\{\left(\vartheta^{\top}+\frac{1}{n} \sum_{i=1}^{n} b_{i}^{\top}\right) \epsilon_{j} X_{k}\right\} \\
& \doteq E\left(\epsilon_{i} \vartheta^{\top} X_{k}\right)+\frac{1}{n} E\left(b^{\top} X\right) E(\epsilon)+\frac{n-2}{n} E(\epsilon) E(b)^{\top} E(X)+\frac{1}{n} E(b \epsilon)^{\top} E(X) \\
& \doteq \frac{1}{n} E(b \epsilon)^{\top} E(X) .
\end{aligned}
$$

By a similar proof,

$$
\begin{aligned}
E\left(\delta_{i} \epsilon_{i} \hat{\vartheta}^{\top} X_{j}\right) & \doteq \frac{1}{n} E(b \delta \epsilon)^{\top} E(X) \\
E\left(\delta_{i} \delta_{j} \epsilon_{i} \hat{\vartheta}^{\top} X_{j}\right) & \doteq \frac{1}{n} E(b \delta \epsilon)^{\top} E(\delta X) .
\end{aligned}
$$

Next is an expected value involving $\hat{\vartheta}^{\top} X X^{\top} \hat{\vartheta}$,

$$
\begin{aligned}
E\left(\hat{\vartheta}^{\top} X X \hat{\vartheta}^{\top}\right) \doteq & E\left\{\left(\vartheta^{\top}+\frac{1}{n} \sum_{i=1}^{n} b_{i}^{\top}\right) X_{j} X_{j}^{\top}\left(\vartheta+\frac{1}{n} \sum_{i=1}^{n} b_{i}\right)\right\} \\
\doteq & E\left(\vartheta^{\top} X X^{\top} \vartheta+2 \vartheta^{\top} \frac{1}{n} \sum_{i=1}^{n} X_{j} X_{j}^{\top} b_{i}+\frac{1}{n^{2}} \sum_{i=1}^{n} \sum_{j=1}^{n} b_{i}^{\top} X_{k} X_{k}^{\top} b_{j}\right) \\
\doteq & \vartheta^{\top} E\left(X X^{\top}\right) \vartheta+2 \vartheta^{\top} \frac{1}{n} E\left(X X^{\top} b\right)+2 \vartheta^{\top} \frac{n-1}{n} E\left(X X^{\top}\right) E(b) \\
& +E\left(\frac{1}{n^{2}} \sum_{i=j}^{\mathrm{n} \text { terms }} b_{i}^{\top} X_{k} X_{k}^{\top} b_{i}+\frac{1}{n^{2}} \sum_{i \neq j}^{n^{2}}{ }^{n \text { terms }} b_{i}^{\top} X_{k} X_{k}^{\top} b_{j}\right) .
\end{aligned}
$$


Using the fact that $E(b)=0$,

$$
\begin{aligned}
E\left(\hat{\vartheta}^{\top} X X^{\top} \hat{\vartheta}\right) \doteq & \vartheta^{\top} E\left(X X^{\top}\right) \vartheta+2 \vartheta^{\top} \frac{1}{n} E\left(X X^{\top} b\right) \\
& +E\left(\frac{1}{n^{2}} b^{\top} X X^{\top} b+\frac{1}{n^{2}} \sum_{i=j \neq k}^{\mathrm{n}-1 \text { terms }} b_{i}^{\top} X_{k} X_{k}^{\top} b_{i}\right. \\
& \left.+\frac{2(n-1)}{n^{2}} b_{i}^{\top} X_{i} X_{i}^{\top} b_{j}+\frac{1}{n^{2}} \sum_{i \neq j \neq k}^{(n-1)(n-2) \text { terms }} b_{i}^{\top} X_{k} X_{k}^{\top} b_{j}\right) \\
\doteq & \vartheta^{\top} E\left(X X^{\top}\right) \vartheta+2 \vartheta^{\top} \frac{1}{n} E\left(X X^{\top} b\right) \\
& +\frac{1}{n^{2}} E\left(b^{\top} X X^{\top} b\right)+\frac{n-1}{n^{2}} E\left(b_{i}^{\top} X_{j} X_{j}^{\top} b_{i}\right) \\
& +\frac{2(n-1)}{n} E\left(b^{\top} X X^{\top}\right) E(b)+\frac{(n-1)(n-2)}{n^{2}} E(b)^{\top} E\left(X X^{\top}\right) E(b) .
\end{aligned}
$$

Again using the $E(b)=0$ and using the fact that any term of order $1 / n^{2}$ can be included in the term $o\left(n^{1 / 2}\right)$ which is noted with the symbol $\doteq$, and denoting the trace of matrix by $T r$, then

$$
\begin{aligned}
E\left(\hat{\vartheta}^{\top} X X^{\top} \hat{\vartheta}\right) \doteq & \vartheta^{\top} E\left(X X^{\top}\right) \vartheta+2 \vartheta^{\top} \frac{1}{n} E\left(X X^{\top} b\right)+\frac{1}{n} E\left(b_{i}^{\top} X_{j} X_{j}^{\top} b_{i}\right) \\
\doteq & \vartheta^{\top} E\left(X X^{\top}\right) \vartheta \\
& +\frac{1}{n}\left[2 \vartheta^{\top} E\left(X X^{\top} b\right)+\operatorname{Tr}\left\{E\left(b b^{\top}\right) E\left(X X^{\top}\right)\right\}\right] .
\end{aligned}
$$

By similar proof

$$
E\left(\delta \hat{\vartheta}^{\top} X X^{\top} \hat{\vartheta}\right) \doteq \vartheta^{\top} E\left(\delta X X^{\top}\right) \vartheta+\frac{1}{n}\left[2 \vartheta^{\top} E\left(\delta X X^{\top} b\right)+\operatorname{Tr}\left\{E\left(b b^{\top}\right) E\left(\delta X X^{\top}\right)\right\}\right]
$$


Another expected value is

$$
\begin{aligned}
E\left(\hat{\vartheta}^{\top} X_{i} X_{j}^{\top} \hat{\vartheta}\right) \doteq & E\left\{\left(\vartheta^{\top}+\frac{1}{n} \sum_{i=1}^{n} b_{i}^{\top}\right) X_{j} X_{k}^{\top}\left(\vartheta+\frac{1}{n} \sum_{i=1}^{n} b_{i}\right)\right\} \\
\doteq & E\left(\vartheta^{\top} X_{i} X_{j}^{\top} \vartheta+\vartheta^{\top} \frac{1}{n} \sum_{i=1}^{n} X_{j} X_{k}^{\top} b_{i}\right. \\
& \left.+\vartheta^{\top} \frac{1}{n} \sum_{i=1}^{n} X_{k} X_{j}^{\top} b_{i}+\frac{1}{n^{2}} \sum_{i=1}^{n} \sum_{j=1}^{n} b_{i}^{\top} X_{k} X_{L}^{\top} b_{j}\right) .
\end{aligned}
$$

The sums will be separated into each scenario as defined in the summation,

$$
\begin{aligned}
E\left(\hat{\vartheta}^{\top} X_{i} X_{j}^{\top} \hat{\vartheta}\right) \doteq & E\left(\vartheta^{\top} X X^{\top} \vartheta+\vartheta^{\top} \frac{2}{n} X_{i} X_{j}^{\top} b_{i}+\vartheta^{\top} \frac{2}{n} X_{j} X_{i}^{\top} b_{i}\right. \\
& +\frac{1}{n^{2}} \sum_{k \neq i=j \neq L}^{\mathrm{n}-2 \text { terms }} b_{i}^{\top} X_{k} X_{L}^{\top} b_{i}+\frac{1}{n^{2}} \sum_{k=i=j}^{2 \text { terms }} b_{i}^{\top} X_{i} X_{k}^{\top} b_{i} \\
& +\frac{1}{n^{2}} b_{i}^{\top} X_{i} X_{j}^{\top} b_{j}+\frac{1}{n^{2}} b_{i}^{\top} X_{j} X_{i}^{\top} b_{j}+\frac{2}{n^{2}} \sum_{k \neq i \neq j=L}^{\mathrm{n}-2 \text { terms }} b_{i}^{\top} X_{i} X_{k}^{\top} b_{j} \\
& \left.+\frac{2}{n^{2}} \sum_{k \neq i \neq j=L}^{\mathrm{n}-2 \text { terms }} b_{i}^{\top} X_{k} X_{i}^{\top} b_{j}+\frac{1}{n^{2}} \sum_{k \neq i \neq j \neq L}^{n^{2}-5 n+6 \text { terms }} b_{i}^{\top} X_{k} X_{L}^{\top} b_{j}\right) .
\end{aligned}
$$

Then the expected values are

$$
\begin{aligned}
E\left(\hat{\vartheta}^{\top} X_{i} X_{j}^{\top} \hat{\vartheta}\right) \doteq & \vartheta^{\top} E(X) E(X)^{\top} \vartheta+\vartheta^{\top} \frac{2}{n} E\left(X b^{\top}\right) E(X)+\vartheta^{\top} \frac{2}{n} E(X) E\left(X^{\top} b\right) \\
& +\frac{n-2}{n^{2}} E(X)^{\top} E\left(b b^{\top}\right) E(X)+\frac{2}{n^{2}} E\left(b^{\top} X b^{\top}\right) E(X) \\
& +\frac{1}{n^{2}} \operatorname{Tr}\left\{E\left(b X^{\top}\right) E\left(X b^{\top}\right)\right\}+\frac{1}{n} \operatorname{Tr}\left\{E\left(b b^{\top}\right) E(X) E(X)^{\top}\right\} \\
& +\frac{2 n-4}{n^{2}} E\left(b^{\top} X\right) E(X)^{\top} E(b)+\frac{2 n-4}{n^{2}} E(X)^{\top} E\left(b X^{\top}\right) E(b) \\
& +\frac{n^{2}-5 n+6}{n^{2}} E(b)^{\top} E(X) E(X)^{\top} E(b) .
\end{aligned}
$$

Now using $E(b)=0$ and the fact that any term or order $1 / n^{2}$ is included in the term 
$o\left(n^{-1 / 2}\right)$ which is denoted by $\doteq$,

$$
\begin{aligned}
E\left(\hat{\vartheta}^{\top} X_{i} X_{j}^{\top} \hat{\vartheta}\right) \doteq & \vartheta^{\top} E(X) E(X)^{\top} \vartheta \\
& +\frac{1}{n}\left\{2 \vartheta^{\top} E\left(X b^{\top}\right) E(X)+2 \vartheta^{\top} E(X) E\left(X^{\top} b\right)\right. \\
& \left.+E(X)^{\top} E\left(b b^{\top}\right) E(X)\right\} .
\end{aligned}
$$

By a similar proof,

$$
\begin{aligned}
E\left(\delta_{i} \hat{\vartheta}^{\top} X_{i} X_{j}^{\top} \hat{\vartheta}\right) \doteq & \vartheta^{\top} E(X) E(\delta X)^{\top} \vartheta+\frac{1}{n}\left\{\vartheta^{\top} E(\delta X) E\left(X^{\top} b\right)\right. \\
& +\vartheta^{\top} E(X) E\left(\delta X^{\top} b\right)+\vartheta^{\top} E\left(X b^{\top}\right) E(\delta X) \\
& \left.+\vartheta^{\top} E\left(\delta X b^{\top}\right) E(X)+E(X)^{\top} E\left(b b^{\top}\right) E(\delta X)\right\} \\
E\left(\delta_{i} \delta_{j} \hat{\vartheta}^{\top} X_{i} X_{j}^{\top} \hat{\vartheta}\right) \doteq & \vartheta^{\top} E(\delta X) E(\delta X)^{\top} \vartheta+\frac{1}{n}\left\{2 \vartheta^{\top} E(\delta X) E\left(\delta X^{\top} b\right)\right. \\
& \left.2 \vartheta^{\top} E\left(\delta X b^{\top}\right) E(\delta X)+E(\delta X)^{\top} E\left(b b^{\top}\right) E(\delta X)\right\} .
\end{aligned}
$$

The asymptotic variance is defined as

$$
\begin{aligned}
A V_{P I}= & \lim _{n \rightarrow \infty} n E\left(\left[\frac{1}{n} \sum_{i=1}^{n}\left\{\delta_{i} Y_{i}+\left(1-\delta_{i}\right) \hat{\vartheta}^{\top} X_{i}\right\}-E(Y)\right]^{2}\right) \\
= & \lim _{n \rightarrow \infty} n E\left[\frac{1}{n^{2}} \sum_{i=1}^{n} \sum_{j=1}^{n}\left\{\delta_{i} Y_{i}+\left(1-\delta_{i}\right) \hat{\vartheta}^{\top} X_{i}\right\}\left\{\delta_{j} Y_{j}+\left(1-\delta_{j}\right) \hat{\vartheta}^{\top} X_{j}\right\}\right] \\
& -n 2 E(Y) \frac{1}{n} \sum_{i=1}^{n}\left\{\delta_{i} Y_{i}+\left(1-\delta_{i}\right) \hat{\vartheta}^{\top} X_{i}\right\}+n E^{2}(Y) .
\end{aligned}
$$


The second half of the equation will be simplified first

$$
\begin{aligned}
A V_{P I}= & \lim _{n \rightarrow \infty}\left(n E\left[\frac{1}{n^{2}} \sum_{i=1}^{n} \sum_{j=1}^{n}\left\{\delta_{i} Y_{i}+\left(1-\delta_{i}\right) \hat{\vartheta}^{\top} X_{i}\right\}\left\{\delta_{j} Y_{j}+\left(1-\delta_{j}\right) \hat{\vartheta}^{\top} X_{j}\right\}\right]\right. \\
& \left.-2 n \vartheta^{\top} E(X) E\left\{\delta_{i} Y_{i}+\left(1-\delta_{i}\right) \hat{\vartheta}^{\top} X_{i}\right\}+n \vartheta^{\top} E(X) E(X)^{\top} \vartheta\right) \\
= & \lim _{n \rightarrow \infty}\left(n E\left[\frac{1}{n^{2}} \sum_{i=1}^{n} \sum_{j=1}^{n}\left\{\delta_{i} Y_{i}+\left(1-\delta_{i}\right) \hat{\vartheta}^{\top} X_{i}\right\}\left\{\delta_{j} Y_{j}+\left(1-\delta_{j}\right) \hat{\vartheta}^{\top} X_{j}\right\}\right]\right. \\
& -2 n \vartheta^{\top} E(X)\left\{E(\delta Y)+E\left(\hat{\vartheta}^{\top} X\right)-E\left(\delta \hat{\vartheta}^{\top} X\right)\right\} \\
& \left.+n \vartheta^{\top} E(X) E(X)^{\top} \vartheta\right) .
\end{aligned}
$$

Now using Equation 3.9

$$
\begin{aligned}
A V_{P I}= & \lim _{n \rightarrow \infty}\left(n E\left[\frac{1}{n^{2}} \sum_{i=1}^{n} \sum_{j=1}^{n}\left\{\delta_{i} Y_{i}+\left(1-\delta_{i}\right) \hat{\vartheta}^{\top} X_{i}\right\}\left\{\delta_{j} Y_{j}+\left(1-\delta_{j}\right) \hat{\vartheta}^{\top} X_{j}\right\}\right]\right. \\
& -2 n \vartheta^{\top} E(X)\left\{\vartheta^{\top} E(\delta X)+\vartheta^{\top} E(X)+\frac{1}{n} E\left(b^{\top} X\right)-\vartheta^{\top} E(\delta X)\right. \\
& \left.\left.-\frac{1}{n} E\left(\delta b^{\top} X\right)\right\}+n \vartheta^{\top} E(X) E(X)^{\top} \vartheta\right) \\
= & \lim _{n \rightarrow \infty}\left(n E\left[\frac{1}{n^{2}} \sum_{i=1}^{n} \sum_{j=1}^{n}\left\{\delta_{i} Y_{i}+\left(1-\delta_{i}\right) \hat{\vartheta}^{\top} X X_{i}\right\}\left\{\delta_{j} Y_{j}+\left(1-\delta_{j}\right) \hat{\vartheta}^{\top} X_{j}\right\}\right]\right. \\
& -2 n \vartheta^{\top} E(X) E(\delta X)^{\top} \vartheta-2 n \vartheta^{\top} E(X) E(X)^{\top} \vartheta \\
& +2 n \vartheta^{\top} E(X) E(\delta X)^{\top} \vartheta+n \vartheta^{\top} E(X) E(X)^{\top} \vartheta \\
& \left.-2 \vartheta^{\top} E(X)\left\{E\left(b^{\top} X\right)-E\left(\delta b^{\top} X\right)\right\}\right) \\
= & \lim _{n \rightarrow \infty}\left(n E\left[\frac{1}{n^{2}} \sum_{i=1}^{n} \sum_{j=1}^{n}\left\{\delta_{i} Y_{i}+\left(1-\delta_{i}\right) \hat{\vartheta}^{\top} X X_{i}\right\}\left\{\delta_{j} Y_{j}+\left(1-\delta_{j}\right) \hat{\vartheta}^{\top} X_{j}\right\}\right]\right. \\
& \left.-n \vartheta^{\top} E(X) E(X)^{\top} \vartheta-2 \vartheta^{\top} E(X)\left\{E\left(b^{\top} X\right)-E\left(\delta b^{\top} X\right)\right\}\right) .
\end{aligned}
$$

Now the double summation will be simplified by breaking it into two scenarios as 
noted by the indicies on the summations,

$$
\begin{aligned}
A V_{P I}= & \lim _{n \rightarrow \infty}\left(n E\left[\frac{1}{n^{2}} \sum_{i=j}^{\mathrm{n} \text { terms }}\left\{\delta_{i} Y_{i}+\left(1-\delta_{i}\right) \hat{\vartheta}^{\top} X_{i}\right\}^{2}\right]\right. \\
& +n E\left[\frac{1}{n^{2}} \sum_{i \neq j}^{n^{2}}\left\{\delta_{i} Y_{i}+\left(1-\delta_{i}\right) \hat{\vartheta}^{\top} X_{i}\right\}\left\{\delta_{j} Y_{j}+\left(1-\delta_{j}\right) \hat{\vartheta}^{\top} X_{j}\right\}\right] \\
& \left.-n \vartheta^{\top} E(X) E(X)^{\top} \vartheta-2 \vartheta^{\top} E(X)\left\{E\left(b^{\top} X\right)-E\left(\delta b^{\top} X\right)\right\}\right) .
\end{aligned}
$$

The first set of terms in Equation 3.14 where $i=j$ will be simplified separately,

$$
\begin{aligned}
& E\left[\frac{1}{n^{2}} \sum_{i=j}^{\mathrm{n} \text { terms }}\left\{\delta_{i} Y_{i}+\left(1-\delta_{i}\right) \hat{\vartheta}^{\top} X_{i}\right\}^{2}\right] \\
= & \frac{n}{n^{2}} E\left(\delta Y^{2}+2 \delta Y \hat{\vartheta}^{\top} X-2 \delta Y \hat{\vartheta}^{\top} X+\hat{\vartheta}^{\top} X X^{\top} \hat{\vartheta}-2 \delta \hat{\vartheta}^{\top} X X^{\top} \hat{\vartheta}+\delta \hat{\vartheta}^{\top} X X^{\top} \hat{\vartheta}\right) \\
= & \frac{1}{n}\left\{E\left(\delta Y^{2}\right)+2 E\left(\delta Y \hat{\vartheta}^{\top} X\right)-2 E\left(\delta Y \hat{\vartheta}^{\top} X\right)+E\left(\hat{\vartheta}^{\top} X X^{\top} \vartheta\right)-E\left(\delta \hat{\vartheta}^{\top} X X^{\top} \vartheta\right)\right\} \\
= & \frac{1}{n}\left[E\left\{\delta\left(\vartheta^{\top} X+\epsilon\right)^{2}\right\}+E\left(\hat{\vartheta}^{\top} X X^{\top} \vartheta\right)-E\left(\delta \hat{\vartheta}^{\top} X X^{\top} \vartheta\right)\right] \\
= & \frac{1}{n}\left\{\vartheta^{\top} E\left(\delta X X^{\top}\right) \vartheta+2 \vartheta^{\top} E(\delta X \epsilon)+E\left(\delta \epsilon^{2}\right)+E\left(\hat{\vartheta}^{\top} X X^{\top} \vartheta\right)-E\left(\delta \hat{\vartheta}^{\top} X X^{\top} \vartheta\right)\right\} \\
= & \frac{1}{n}\left\{\vartheta^{\top} E(\delta X) E(\delta X)^{\top} \vartheta+\sigma^{2} E(\delta)+E\left(\hat{\vartheta}^{\top} X X^{\top} \hat{\vartheta}\right)-E\left(\delta \hat{\vartheta}^{\top} X X^{\top} \hat{\vartheta}\right)\right\} .
\end{aligned}
$$

Using Equation 3.12 ,

$$
\begin{aligned}
& E\left[\frac{1}{n} \sum_{i=j}^{\mathrm{n} \text { terms }}\left\{\delta_{i} Y_{i}+\left(1-\delta_{i}\right) \hat{\vartheta}^{\top} X_{i}\right\}^{2}\right] \\
\doteq & \frac{1}{n}\left(\vartheta^{\top} E\left(\delta X X^{\top}\right) \vartheta+\sigma^{2} E(\delta)+\vartheta^{\top} E\left(X X^{\top}\right) \vartheta+\right. \\
& \frac{1}{n}\left[2 \vartheta^{\top} E\left(X X^{\top} b\right)+\operatorname{Tr}\left\{E\left(b b^{\top}\right) E\left(X X^{\top}\right)\right\}\right] \\
& \left.-\vartheta^{\top} E\left(\delta X X^{\top}\right) \vartheta-\frac{1}{n}\left[2 \vartheta^{\top} E\left(\delta X X^{\top} b\right)+\operatorname{Tr}\left\{E\left(b b^{\top}\right) E\left(\delta X X^{\top}\right)\right\}\right]\right) .
\end{aligned}
$$

Since terms of order $1 / n^{2}$ can be included in the term $o_{p}\left(n^{-1 / 2}\right)$ which is denoted by 
the symbol $\doteq$

$$
\begin{aligned}
& E\left[\frac{1}{n} \sum_{i=j}^{\mathrm{n} \text { terms }}\left\{\delta_{i} Y_{i}+\left(1-\delta_{i}\right) \hat{\vartheta}^{\top} X_{i}\right\}^{2}\right] \\
\doteq & \frac{1}{n}\left\{\vartheta^{\top} E\left(\delta X X^{\top}\right) \vartheta+\sigma^{2} E(\delta)+\vartheta^{\top} E\left(X X^{\top}\right) \vartheta-\vartheta^{\top} E\left(\delta X X^{\top}\right) \vartheta\right\} \\
\doteq & \frac{1}{n}\left\{\sigma^{2} E(\delta)+\vartheta^{\top} E\left(X X^{\top}\right) \vartheta\right\} .
\end{aligned}
$$

Now the second set of summations used in Equation 3.14 will be simplified,

$$
\begin{aligned}
& E\left[\frac{1}{n^{2}} \sum_{i \neq j}^{n^{2}-n \text { terms }}\left\{\delta_{i} Y_{i}+\left(1-\delta_{i}\right) \hat{\vartheta}^{\top} X_{i}\right\}\left\{\delta_{j} Y_{j}+\left(1-\delta_{j}\right) \hat{\vartheta}^{\top} X_{j}\right\}\right] \\
= & \frac{1}{n^{2}} E\left\{\sum _ { i \neq j } ^ { n ^ { 2 } - n \text { terms } } \left(\delta_{i} \delta_{j} Y_{i} Y_{j}+2 \delta_{i} Y_{i} \hat{\vartheta}^{\top} X_{j}-2 \delta_{i} \delta_{j} Y_{i} \hat{\vartheta}^{\top} X_{j}+\hat{\vartheta}^{\top} X_{i} X_{j}^{\top} \hat{\vartheta}\right.\right. \\
& \left.\left.-2 \delta_{j} \hat{\vartheta}^{\top} X_{i} X_{j}^{\top} \hat{\vartheta}+\delta_{i} \delta_{j} \hat{\vartheta}^{\top} X_{i} X_{j}^{\top} \hat{\vartheta}\right)\right\} \\
= & \frac{n^{2}-n}{n^{2}}\left\{E^{2}(\delta Y)+2 \vartheta^{\top} E\left(\delta_{i} X_{i} X_{j}^{\top} \hat{\vartheta}\right)+2 E\left(\delta_{i} \epsilon_{i} \hat{\vartheta}^{\top} X_{j}\right)-2 \vartheta^{\top} E\left(\delta_{i} \delta_{j} X_{i} X_{j}^{\top} \hat{\vartheta}\right)\right. \\
& \left.-2 E\left(\delta_{i} \delta_{j} \epsilon_{i} \hat{\vartheta}^{\top} X_{j}\right)+E\left(\hat{\vartheta}^{\top} X_{i} X_{j}^{\top} \hat{\vartheta}\right)-2 E\left(\delta_{j} \hat{\vartheta}^{\top} X_{i} X_{j}^{\top} \hat{\vartheta}\right)+E\left(\delta_{i} \delta_{j} \hat{\vartheta}^{\top} X_{i} X_{j}^{\top} \hat{\vartheta}\right)\right\}
\end{aligned}
$$


Now using the expectations found in Equation 3.10, Equation 3.11, and Equation 3.13,

$$
\begin{aligned}
& E\left[\frac{1}{n^{2}} \sum_{i \neq j}^{n^{2}-n \text { terms }}\left\{\delta_{i} Y_{i}+\left(1-\delta_{i}\right) \hat{\vartheta}^{\top} X_{i}\right\}\left\{\delta_{j} Y_{j}+\left(1-\delta_{j}\right) \hat{\vartheta}^{\top} X_{j}\right\}\right] \\
\doteq & \frac{n-1}{n}\left(\vartheta^{\top} E(\delta X) E(\delta X)^{\top} \vartheta+2 \vartheta^{\top}\left\{E(\delta X) E(X)^{\top} \vartheta+\frac{1}{n} E(\delta X) E\left(X^{\top} b\right)\right.\right. \\
& \left.+\frac{1}{n} E\left(\delta X b^{\top}\right) E(X)\right\}+2\left\{\frac{1}{n} E(b \delta \epsilon)^{\top} E(X)\right\}-2 \vartheta^{\top}\left\{E(\delta X) E(\delta X)^{\top} \vartheta\right. \\
& \left.+\frac{1}{n} E(\delta X) E\left(\delta X^{\top} b\right)+\frac{1}{n} E\left(\delta X b^{\top}\right) E(\delta X)\right\}-2\left\{\frac{1}{n} E(b \delta \epsilon)^{\top} E(\delta X)\right\} \\
& +\left[\vartheta^{\top} E(X) E(X)^{\top} \vartheta+\frac{1}{n} 2 \vartheta^{\top} E(X) E\left(X^{\top} b\right)+2 \frac{1}{n} \vartheta^{\top} E\left(X b^{\top}\right) E(X)\right. \\
& \left.+\frac{1}{n} E(X)^{\top} E\left(b b^{\top}\right) E(X)\right]-2\left\{\vartheta^{\top} E(X) E(\delta X)^{\top} \vartheta+\frac{1}{n} \vartheta^{\top} E(X) E\left(\delta X^{\top} b\right)\right. \\
& +\frac{1}{n} \vartheta^{\top} E\left(\delta X b^{\top}\right) E(X)+\frac{1}{n} \vartheta^{\top} E(\delta X) E\left(X^{\top} b\right)+\frac{1}{n} \vartheta^{\top} E\left(X b^{\top}\right) E(\delta X) \\
& \left.+\frac{1}{n} E(X)^{\top} E\left(b b^{\top}\right) E(\delta X)\right\}+\left\{\vartheta^{\top} E(\delta X) E(\delta X)^{\top} \vartheta+\frac{1}{n} 2 \vartheta^{\top} E(\delta X) E\left(\delta X^{\top} b\right)\right. \\
& \left.\left.+\frac{1}{n} 2 \vartheta^{\top} E\left(\delta X b^{\top}\right) E(\delta X)+\frac{1}{n} E(\delta X)^{\top} E\left(b b^{\top}\right) E(\delta X)\right\}\right) .
\end{aligned}
$$

Since items of order $1 / n^{2}$ can be included in the term $o_{p}\left(n^{-1 / 2}\right)$ which is denoted 
using the symbol $\doteq$,

$$
\begin{aligned}
& E\left[\frac{1}{n^{2}} \sum_{i \neq j}^{n^{2}-n \text { terms }}\left\{\delta_{i} Y_{i}+\left(1-\delta_{i}\right) \hat{\vartheta}^{\top} X_{i}\right\}\left\{\delta_{j} Y_{j}+\left(1-\delta_{j}\right) \hat{\vartheta}^{\top} X_{j}\right\}\right] \\
\doteq & \vartheta^{\top} E(\delta X) E(\delta X)^{\top} \vartheta+2 \vartheta^{\top} E(\delta X) E(X)^{\top} \vartheta-2 \vartheta^{\top} E(\delta X) E(\delta X)^{\top} \vartheta \\
& +\vartheta^{\top} E(X) E(X)^{\top} \vartheta-2 \vartheta^{\top} E(X) E(\delta X)^{\top} \vartheta+\vartheta^{\top} E(\delta X) E(\delta X)^{\top} \vartheta \\
& +\frac{1}{n}\left[-\left\{\vartheta^{\top} E(\delta X) E(\delta X)^{\top} \vartheta+2 \vartheta^{\top} E(\delta X) E(X)^{\top} \vartheta-2 \vartheta^{\top} E(\delta X) E(\delta X)^{\top} \vartheta\right.\right. \\
& \left.+\vartheta^{\top} E(X) E(X)^{\top} \vartheta-2 \vartheta^{\top} E(X) E(\delta X)^{\top} \vartheta+\vartheta^{\top} E(\delta X) E(\delta X)^{\top} \vartheta\right\} \\
& +2 \vartheta^{\top} E(\delta X) E\left(X^{\top} b\right)+2 \vartheta^{\top} E\left(\delta X b^{\top}\right) E(X)+2 E(b \delta \epsilon)^{\top} E(X) \\
& -2 \vartheta^{\top} E(\delta X) E\left(\delta X^{\top} b\right)-2 \vartheta^{\top} E\left(\delta X b^{\top}\right) E(\delta X)-2 E(b \delta \epsilon)^{\top} E(\delta X) \\
& +2 \vartheta^{\top} E(X) E\left(X^{\top} b\right)+2 \vartheta^{\top} E\left(X b^{\top}\right) E(X)+E(X)^{\top} E\left(b b^{\top}\right) E(X) \\
& -2 \vartheta^{\top} E(X) E\left(\delta X^{\top} b\right)-2 \vartheta^{\top} E\left(\delta X b^{\top}\right) E(X)-2 \vartheta^{\top} E(\delta X) E\left(X^{\top} b\right) \\
& -2 \vartheta^{\top} E\left(X b^{\top}\right) E(\delta X)-2 E(X)^{\top} E\left(b b^{\top}\right) E(\delta X)+2 \vartheta^{\top} E(\delta X) E\left(\delta X{ }^{\top} b\right) \\
& \left.+2 \vartheta^{\top} E\left(\delta X b^{\top}\right) E(\delta X)+E(\delta X)^{\top} E\left(b b^{\top}\right) E(\delta X)\right] .
\end{aligned}
$$

This simplifies to

$$
\begin{aligned}
& E\left[\frac{1}{n^{2}} \sum_{i \neq j}^{n^{2}-n \text { terms }}\left\{\delta_{i} Y_{i}+\left(1-\delta_{i}\right) \hat{\vartheta}^{\top} X_{i}\right\}\left\{\delta_{j} Y_{j}+\left(1-\delta_{j}\right) \hat{\vartheta}^{\top} X_{j}\right\}\right] \\
\doteq & \vartheta^{\top} E(X) E(X)^{\top} \vartheta+\frac{1}{n}\left[-\vartheta^{\top} E(X) E(X)^{\top} \vartheta\right. \\
& +2\left\{E(b \delta \epsilon)^{\top}+\vartheta^{\top} E\left(X b^{\top}\right)\right\}\{E(X)-E(\delta X)\} \\
& +2 \vartheta^{\top} E(X)\left\{E\left(X^{\top} b\right)-E\left(\delta X^{\top} b\right)\right\} \\
& \left.+\{E(X)-E(\delta X)\}^{\top} E\left(b b^{\top}\right)\{E(X)-E(\delta X)\}\right]
\end{aligned}
$$


Now putting Equation 3.16 and Equation 3.15 into Equation 3.14 gives

$$
\begin{aligned}
A V_{P I}= & \lim _{n \rightarrow \infty}\left(\left\{\sigma^{2} E(\delta)+\vartheta^{\top} E\left(X X^{\top}\right) \vartheta\right\}\right. \\
& +n \vartheta^{\top} E(X) E(X)^{\top} \vartheta+\left[-\vartheta^{\top} E(X) E(X)^{\top} \vartheta\right. \\
& +2\left\{E(b \delta \epsilon)^{\top}+\vartheta^{\top} E\left(X b^{\top}\right)\right\}\{E(X)-E(\delta X)\} \\
& +2 \vartheta^{\top} E(X)\left\{E\left(X^{\top} b\right)-E\left(\delta X^{\top} b\right)\right\} \\
& \left.+\{E(X)-E(\delta X)\}^{\top} E\left(b b^{\top}\right)\{E(X)-E(\delta X)\}\right] \\
& \left.-n \vartheta^{\top} E(X) E(X)^{\top} \vartheta-2 \vartheta^{\top} E(X)\left\{E\left(X^{\top} b\right)-E\left(\delta X^{\top} b\right)\right\}\right) .
\end{aligned}
$$

This simplifies to

$$
\begin{aligned}
A V_{P I}= & \vartheta^{\top}\left\{E\left(X X^{\top}\right)-E(X) E(X)^{\top}\right\} \vartheta+\sigma^{2} E(\delta) \\
& +\left\{E(b \delta \epsilon)^{\top}+\vartheta^{\top} E\left(X b^{\top}\right)\right\}\{E(X)-E(\delta X)\} \\
& +\{E(X)-E(\delta X)\}^{\top} E\left(b b^{\top}\right)\{E(X)-E(\delta X)\} .
\end{aligned}
$$

This variance will be compared with full imputation in Chapter IV and compared with other methods in Chapter $\mathrm{V}$.

\section{Full imputation}

Full imputation incorporates all the information about the model and imputes all the data, even data which are not missing. Full imputation does not imply that data are erased as the observed responses are used in estimating $\vartheta$. As shown in Müller (2009) the formula for full imputation requires weights which account for the error structure. To find the final form of the estimate begin with the equation for full 
imputation adjusted for the estimation of $E(Y)$,

$$
\widehat{E[Y]_{F I}}=\frac{1}{n} \sum_{i=1}^{n} \frac{\sum_{j=1}^{n} \hat{w}_{j} \delta_{j}\left(\hat{\vartheta}^{\top} X_{i}+Y_{j}-\hat{\vartheta}^{\top} X_{j}\right)}{\sum_{j=1}^{n} \delta_{i}}
$$

where $\hat{\epsilon}=Y-\hat{\vartheta}^{\top} X, \hat{w}_{j}>0$, and it satisfies the conditions $\sum_{j=1}^{n} \hat{w}_{j} \delta_{j} \hat{\epsilon}_{j}=0$ and $\sum_{j=1}^{n} \hat{w}_{j}=n$. As shown by Owen (2001), these weights can be found by

$$
\hat{w}_{j}=\frac{1}{1+\lambda \delta_{j} \hat{\epsilon}} .
$$

$\lambda$ is solved by finding the solution to

$$
\sum_{j=1}^{n} \frac{\delta_{j} \hat{\epsilon}_{j}}{1+\lambda \delta_{j} \hat{\epsilon}_{j}}=0
$$

Thus when estimating $E(Y)$ the fully imputed estimator given in Equation 3.17 simplifies to

$$
\widehat{E[Y]_{F I}}=\frac{1}{n} \sum_{i=1}^{n} \hat{\vartheta}^{\top} X_{i}
$$

The following theorem shows that full imputation is unbiased

Theorem III.4 The expected value for full imputation is approximately

$$
E\left(\widehat{E(Y)_{F I}}\right)=E(Y)
$$


PRoOF: Using the expectation found in Equation 3.9,

$$
\begin{aligned}
E\left(\widehat{E(Y)_{F I}}\right) & =E\left\{\frac{1}{n} \sum_{i=1}^{n} \hat{\vartheta}^{\top} X_{i}\right\} \\
& =E\left(\hat{\vartheta}^{\top} X_{i}\right) \\
& =\vartheta^{\top} E(X)+\frac{1}{n} E\left(b^{\top} X\right) \\
& \doteq E\left(\vartheta^{\top} X+\epsilon\right) \\
& \doteq E(Y) .
\end{aligned}
$$

Now the asymptotic variance of the fully imputed estimator will be shown.

Theorem III.5 The asymptotic variance for full imputation is

$$
\begin{aligned}
A V_{F I}= & \vartheta^{\top}\left\{E\left(X X^{\top}\right)-E(X) E(X)^{\top}\right\} \vartheta+2 \vartheta^{\top} E\left(X b^{\top}\right) E(X) \\
& +2 \vartheta^{\top} E(X) E\left(X^{\top} b\right)+E(X)^{\top} E\left(b b^{\top}\right) E(X) .
\end{aligned}
$$

PRoOF: The asymptotic variance is defined as

$$
\begin{aligned}
A V_{F I} & =\lim _{n \rightarrow \infty} n E\left[\left\{\frac{1}{n} \sum_{i=1}^{n} \hat{\vartheta}^{\top} X_{i}-E(Y)\right\}^{2}\right] \\
& =\lim _{n \rightarrow \infty} n E\left\{\frac{1}{n^{2}} \sum_{i=1}^{n} \sum_{j=1}^{n} \hat{\vartheta}^{\top} X_{i} X_{j}^{\top} \vartheta-2 E(Y) \frac{1}{n} \sum_{i=1}^{n} \hat{\vartheta}^{\top} X_{i}+E^{2}(Y)\right\} \\
& =\lim _{n \rightarrow \infty} n E\left[\frac{1}{n^{2}} \sum_{i=1}^{n} \sum_{j=1}^{n} \hat{\vartheta}^{\top} X_{i} X_{j}^{\top} \vartheta-2 E(Y)\left\{E(Y)+\frac{1}{n} E\left(X^{\top} b\right)\right\}+E^{2}(Y)\right] \\
& =\lim _{n \rightarrow \infty} n E\left\{\frac{1}{n^{2}} \sum_{i=1}^{n} \sum_{j=1}^{n} \hat{\vartheta}^{\top} X_{i} X_{j}^{\top} \vartheta-\vartheta^{\top} E(X) E(X)^{\top} \vartheta-2 \frac{1}{n} \vartheta^{\top} E(X) E\left(X^{\top} b\right)\right\}
\end{aligned}
$$


The double summations will be split using the same notation as before

$$
\begin{aligned}
A V_{F I}= & \lim _{n \rightarrow \infty} n E\left\{\frac{1}{n^{2}} \sum_{i=j}^{\mathrm{n} \text { terms }} \hat{\vartheta}^{\top} X_{i} X_{i}^{\top} \vartheta+\frac{1}{n^{2}} \sum_{i \neq j}^{\mathrm{n}(\mathrm{n}-1) \text { terms }} \hat{\vartheta}^{\top} X_{i} X_{j}^{\top} \vartheta\right. \\
& \left.-\vartheta^{\top} E(X) E(X)^{\top} \vartheta-2 \frac{1}{n} \vartheta^{\top} E(X) E\left(X^{\top} b\right)\right\} .
\end{aligned}
$$

Using Equation 3.12 and Equation 3.13 ,

$$
\begin{aligned}
A V_{F I}= & \lim _{n \rightarrow \infty}\left\{E\left(\hat{\vartheta}^{\top} X_{i} X_{i}^{\top} \hat{\vartheta}\right)+(n-1) E\left(\hat{\vartheta}^{\top} X_{i} X_{j}^{\top} \hat{\vartheta}\right)-n \vartheta^{\top} E(X) E(X)^{\top} \vartheta\right\} \\
= & \lim _{n \rightarrow \infty}\left\{\left(\vartheta^{\top} E\left(X X^{\top}\right) \vartheta+\frac{1}{n}\left[2 \vartheta^{\top} E\left(X X^{\top} b\right)+T r\left\{E\left(b b^{\top}\right) E\left(X X^{\top}\right)\right\}\right]\right)\right. \\
& +(n-1)\left[\vartheta^{\top} E(X) E(X)^{\top} \vartheta+\frac{1}{n}\left\{2 \vartheta^{\top} E\left(X b^{\top}\right) E(X)+2 \vartheta^{\top} E(X) E\left(X^{\top} b\right)\right.\right. \\
& \left.\left.\left.+E(X)^{\top} E\left(b b^{\top}\right) E(X)\right\}\right]-n \vartheta^{\top} E(X) E(X)^{\top} \vartheta-2 \vartheta^{\top} E(X) E\left(X^{\top} b\right)\right\} .
\end{aligned}
$$

The terms of order $1 / n$ asymptotically approach 0 with probability 1 , so

$$
\begin{aligned}
A V_{F I}= & \vartheta^{\top}\left\{E\left(X X^{\top}\right)-E(X) E(X)^{\top}\right\} \vartheta+2 \vartheta^{\top} E\left(X b^{\top}\right) E(X) \\
& +E(X)^{\top} E\left(b b^{\top}\right) E(X) .
\end{aligned}
$$

This variance will be compared with partial imputation in Chapter IV, In Chapter V full imputation will be compared with the other estimators discussed earlier. It will be seen that full imputation has the least amount of asymptotic variance when an efficient estimator of $\vartheta$ is used. 


\section{CHAPTER IV}

\section{COMPARISON OF PARTIAL AND FULL IMPUTATION}

An estimate is considered efficient if the asymptotically linear form of the estimator matches the efficient influence function as defined in Equation 2.1. Müller (2009) shows that full imputation with an efficient (regular) estimator of $\vartheta$ is an ideal method as it is guaranteed to be efficient. The same article in shows in Equation 4.10 what the efficient influence function would be for the parametric model when estimating a generic function $h(X, Y)$. In this paper we have a linear model so the efficient influence function for estimating $E\{h(X, Y)\}=E(Y)$ can be written as

$$
\begin{aligned}
& n^{1 / 2}\{\widehat{E(Y)}-E(Y)\} \\
= & n^{-1 / 2} \sum_{i=1}^{n}\left[\vartheta^{\top} X_{i}-\vartheta^{\top} E(X)\right. \\
& \left.+E(X)^{\top} E\left\{\delta \zeta(\delta, X, \epsilon) \zeta(\delta, X, \epsilon)^{\top}\right\}^{-1} \delta_{i} \zeta\left(\delta_{i}, X_{i}, \epsilon_{i}\right)\right]+o_{p}(1)
\end{aligned}
$$

with $\zeta(\delta, X, \epsilon)$ as defined in Equation 2.2 .

Partial Imputation is perhaps more intuitive since only the data that are missing are imputed. The asymptotic variance of each method is determined by the influence function of the estimator used. In this section we explore how the asymptotic variance is affected by an inefficient estimator of $\vartheta$. 
A. Efficient estimate for $\vartheta$

As shown in Lemma \.1 when an efficient estimator for $\vartheta$ is used the influence function is

$$
\begin{aligned}
b\left(\delta_{i}, X_{i}, \epsilon_{i}\right)=E(\delta \quad & {\left[\{X-E(X \mid \delta=1)\} l(\epsilon)+E(X \mid \delta=1) \frac{\epsilon}{\sigma^{2}}\right] } \\
& {\left.\left[\{X-E(X \mid \delta=1)\} l(\epsilon)+E(X \mid \delta=1) \frac{\epsilon}{\sigma^{2}}\right]^{\top}\right)^{-1} } \\
& \delta_{i}\left[\left\{X_{i}-E(X \mid \delta=1)\right\} l\left(\epsilon_{i}\right)+E(X \mid \delta=1) \frac{\epsilon_{i}}{\sigma^{2}}\right]
\end{aligned}
$$

Using the fact that $\delta$ is either 0 or 1 , the following simplification can be used:

$$
E(X \mid \delta=1)=\frac{E(1 X \mid \delta=1) E(\delta)+E(0 X \mid \delta=0)\{1-E(\delta)\}}{E(\delta)}=\frac{E(\delta X)}{E(\delta)} .
$$

Which means the influence function can be written in a simplified form as

$$
b=E\left(\delta \zeta \zeta^{\top}\right)^{-1} \delta \zeta
$$

where

$$
\zeta=X l(\epsilon)-\frac{E(\delta X)}{E(\delta)} l(\epsilon)+\frac{\epsilon}{\sigma^{2}} \frac{E(\delta X)}{E(\delta)} .
$$

In this case $E\left(X b^{\top}\right)=0, E\left(\delta X b^{\top}\right)=0$, and

$$
\begin{aligned}
E\left(b b^{\top}\right) & =E\left\{E\left(\delta \zeta \zeta^{\top}\right)^{-1} \delta \zeta \zeta^{\top} E\left(\delta \zeta \zeta^{\top}\right)^{-1}\right\} \\
& =E\left(\delta \zeta \zeta^{\top}\right)^{-1} .
\end{aligned}
$$


Also

$$
\begin{aligned}
E(b \delta \epsilon) & =E\left[E\left(\delta \zeta \zeta^{\top}\right)^{-1} \delta\left\{X \epsilon l(\epsilon)-\frac{E(\delta X)}{E(\delta)} \epsilon l(\epsilon)+\frac{\epsilon^{2}}{\sigma^{2}} \frac{E(\delta X)}{E(\delta)}\right\}\right] \\
& =E\left(\delta \zeta \zeta^{\top}\right)^{-1}\{E(\delta X)-E(\delta X)+E(\delta X)\} \\
& =E\left(\delta \zeta \zeta^{\top}\right)^{-1} E(\delta X) .
\end{aligned}
$$

It is important to note that $E\left(\delta \zeta \zeta^{\top}\right)$ depends on the fishers information, $\mathbb{I}$, since

$$
\begin{aligned}
E\left(\delta \zeta \zeta^{\top}\right)= & E\left\{\delta X X^{\top} l^{2}(\epsilon)-\delta X \frac{E(\delta X)^{\top}}{E(\delta)} l^{2}(\epsilon)-\delta \frac{E(\delta X)}{E(\delta)} X^{\top} l^{2}(\epsilon)\right. \\
& +\delta X \frac{E(\delta X)^{\top}}{E(\delta)} \frac{1}{\sigma^{2}} \epsilon l(\epsilon)+\delta \frac{E(\delta X)}{E(\delta)} X^{\top} \frac{1}{\sigma^{2}} \epsilon l(\epsilon)+\delta \frac{E(\delta X) E(\delta X)^{\top}}{E^{2}(\delta)} l^{2}(\epsilon) \\
& -\frac{1}{\sigma^{2}} \delta \frac{E(\delta X) E(\delta X)^{\top}}{E^{2}(\delta)} \epsilon l(\epsilon)-\frac{1}{\sigma^{2}} \delta \frac{E(\delta X) E(\delta X)^{\top}}{E^{2}(\delta)} \epsilon l(\epsilon) \\
& +\delta \frac{\epsilon^{2}}{\left(\sigma^{2}\right)^{2}} \frac{E(\delta X) E(\delta X)^{\top}}{E^{2}(\delta)} \\
= & E\left(\delta X X^{\top}\right) \mathbb{I}-\frac{E(\delta X) E(\delta X)^{\top}}{E(\delta)} \mathbb{I}-\frac{E(\delta X) E(\delta X)^{\top}}{E(\delta)} \mathbb{I} \\
& +\frac{E(\delta X) E(\delta X)^{\top} \frac{1}{\sigma^{2}}+\frac{E(\delta X) E(\delta X)^{\top}}{E(\delta)} \frac{1}{\sigma^{2}}+\frac{E(\delta X) E(\delta X)^{\top}}{E(\delta)} \mathbb{I}}{E(\delta)} \\
& -\frac{1}{\sigma^{2}} \frac{E(\delta X) E(\delta X)^{\top}}{E(\delta)}-\frac{1}{\sigma^{2}} \frac{E(\delta X) E(\delta X)^{\top}}{E(\delta)}+\frac{1}{\sigma^{2}} \frac{E(\delta X) E(\delta X)^{\top}}{E(\delta)} \\
= & \left.E(\delta X X)^{\top}\right) \mathbb{I}-\frac{E(\delta X) E(\delta X)^{\top}}{E(\delta)} \mathbb{I}+\frac{E(\delta X) E(\delta X)^{\top}}{E} \frac{1}{\sigma^{2}} .
\end{aligned}
$$

Then the asymptotic variance for partial imputation is

$$
\begin{aligned}
A V_{P I_{-} E F F}= & \vartheta^{\top}\left\{E\left(X X^{\top}\right)-E(X) E(X)^{\top}\right\} \vartheta+\sigma^{2} E(\delta) \\
& +2 E(\delta X)^{\top} E\left(\delta \zeta \zeta^{\top}\right)^{-1}\{E(X)-E(\delta X)\} \\
& +\{E(X)-E(\delta X)\}^{\top} E\left(\delta \zeta \zeta^{\top}\right)^{-1}\{E(X)-E(\delta X)\} \\
= & \vartheta^{\top}\left\{E\left(X X^{\top}\right)-E(X) E(X)^{\top}\right\} \vartheta+\sigma^{2} E(\delta) \\
& +E(X)^{\top} E\left(\delta \zeta \zeta^{\top}\right)^{-1} E(X)-E(\delta X)^{\top} E\left(\delta \zeta \zeta^{\top}\right)^{-1} E(\delta X) .
\end{aligned}
$$


The asymptotic variance for full imputation is

$$
A V_{F I_{-E F F}}=\vartheta^{\top}\left\{E\left(X X^{\top}\right)-E(X) E(X)^{\top}\right\} \vartheta+E(X)^{\top} E\left(\delta \zeta \zeta^{\top}\right)^{-1} E(X)
$$

It should be noted that this same asymptotic variance can be calculated by taking the expected value of the squared efficient influence function in Equation 4.1.

The difference between the fully imputed estimator and the partially imputed estimator is

$$
\begin{aligned}
& A V_{P I_{-E F F}}-A V_{F I-E F F} \\
= & \sigma^{2} E(\delta)-E(\delta X) E\left(\delta \zeta \zeta^{\top}\right)^{-1} E(\delta X) \\
= & \left(\sigma^{2}\right)^{2} E(\delta) \mathbb{I}\left\{E(\delta)-E(\delta X)^{\top} E\left(\delta X X^{\top}\right)^{-1} E(\delta X)\right\} \\
& {\left[\sigma^{2} \mathbb{I}\left\{E(\delta)-E(\delta X)^{\top} E\left(\delta X X^{\top}\right)^{-1} E(\delta X)\right\}+E(\delta X)^{\top} E\left(\delta X X^{\top}\right)^{-1} E(\delta X)\right]^{-1} }
\end{aligned}
$$

To simplify the notation let

$$
C_{0}=E(\delta)-E(\delta X)^{\top} E\left(\delta X X^{\top}\right)^{-1} E(\delta X)
$$

and

$$
Q_{0}=E(\delta X)^{\top} E\left(\delta X X^{\top}\right)^{-1} E(\delta X)
$$

To show that $C_{0}$ is nonnegative see the discussion after Equation (2.7) in Müller (2007) to apply the Cauchy Schwarz inequality to higher dimensions. The quantity $Q_{0}$ is nonnegative because it has a quadratic form. The difference for the fully imputed estimator and the partially imputed estimator can now be written as

$$
\begin{aligned}
A V_{P I \_E F F}-A V_{F I \_E F F} & =\sigma^{2} E(\delta)-E(\delta X) E\left(\delta \zeta \zeta^{\top}\right)^{-1} E(\delta X) \\
& =\left(\sigma^{2}\right)^{2} E(\delta) \mathbb{I} C_{0}\left(\sigma^{2} \mathbb{I} C_{0}+Q_{0}\right)^{-1} .
\end{aligned}
$$

This difference is always nonnegative which implies partial imputation has at least 
as much asymptotic variance as full imputation when an efficient estimate for $\vartheta$ is used. This supports what the Hajek-Le Cam theory shows in the paper from Müller (2009), which is that full imputation with an efficient estimate for $\vartheta$ is efficient.

B. Weighted least squares estimate of $\vartheta$

The model $E(Y \mid X)=\vartheta^{\top} X$ suggests estimators for $\hat{\vartheta}$ that solve the equation

$$
\sum_{i=1}^{n} \delta_{i} w_{\hat{\vartheta}}\left(X_{i}\right)\left(Y_{i}-\hat{\vartheta}^{\top} X_{i}\right)=0
$$

where $w_{\hat{\vartheta}}$ is a $p$-dimensional vector of weight functions. Note that $E\left\{\delta w_{\vartheta}(X)(Y-\right.$ $\left.\left.\vartheta^{\top} X\right)\right\}=0$.

The next step is to determine the asymptotic linear form of the weighted least squares estimator. Assumptions IV.1 and IV.2 as well as Theorem IV.3 are based on Section 2 of a paper by Müller (2007).

Assumption IV.1 The p-dimensional vector $w_{\tau}(X)$ is $L_{2}(P)$ differentiable at $\tau=\vartheta$ with a $p \times p$ matrix of partial derivatives $\dot{w}_{\vartheta}(X)$ and a p-dimensional gradient $X$, respectively,

$$
E\left\{\left|w_{\tau}(X)-w_{\vartheta}(X)-\dot{w}_{\vartheta}(X)(\tau-\vartheta)\right|^{2}\right\}=o\left(|\tau-\vartheta|^{2}\right)
$$

Assumption IV.1 guarantees that the expected value of $w_{\tau}(X)\left(Y-\tau^{\top} X\right)$ can be approximated as follows,

$$
\begin{aligned}
& E\left\{\delta w_{\tau}(X)\left(Y-\tau^{\top} X\right)\right\}-E\left[\delta w_{\vartheta}(X)\left\{Y-\vartheta^{\top} X\right\}\right] \\
= & -A(\tau-\vartheta)+o(|\tau-\vartheta|),
\end{aligned}
$$

where $A$ is a $p \times p$ matrix of expectations, namely

$$
A=E\left\{\delta w_{\vartheta}(X) X^{\top}\right\}
$$


Assumption IV.2 $A$ is invertible.

By Assumption IV.1, $w_{\tau}$ is $L_{2}(P)$ differentiable. This implies that the empirical process

$$
E_{n \tau}=n^{-1 / 2} \sum_{i=1}^{n}\left[\delta_{i} w_{\tau}\left(X_{i}\right)\left(Y_{i}-\tau^{\top} X_{i}\right)-E\left\{\delta w_{\tau}(X)\left(Y-\tau^{\top} X\right)\right\}\right]
$$

is stochastically equicontinuous at $\tau=\vartheta$ : for every $\varepsilon, \eta>0$ there is a $\delta$ such that

$$
\limsup _{n} P\left(\sup _{|\tau-\vartheta| \leq \delta}\left|E_{n \tau}-E_{n \vartheta}\right|>\eta\right) \leq \varepsilon
$$

See for example Andrews and Pollard (1994) or Müller et al. (2004).

Theorem IV.3 Any consistent solution $\hat{\vartheta}$ of Equation 4.7 has the stochastic expansion

$$
\begin{aligned}
& n^{1 / 2}(\hat{\vartheta}-\vartheta) \\
= & \left\{E\left(\delta w_{\vartheta}(X) X^{\top}\right)\right\}^{-1} n^{-1 / 2} \sum_{i=1}^{n} \delta_{i} w_{\vartheta}\left(X_{i}\right)\left(Y_{i}-\vartheta^{\top} X_{i}\right)+o_{p}(1) .
\end{aligned}
$$

Proof: Consider the estimating Equation 4.7 and the empirical process $E_{n \tau}$ from Equation 4.10 in the above remark. We have

$$
\begin{aligned}
0 & =n^{-1 / 2} \sum_{i=1}^{n} \delta_{i} w_{\hat{\vartheta}}\left(X_{i}\right)\left(Y_{i}-\hat{\vartheta}^{\top} X_{i}\right) \\
& =E_{n \hat{\vartheta}}+n^{-1 / 2} \sum_{i=1}^{n} E\left\{\delta w_{\hat{\vartheta}}(X)\left(Y-\hat{\vartheta}^{\top} X\right)\right\}+E_{n \vartheta}-E_{n \vartheta}
\end{aligned}
$$


with $E_{n \hat{\vartheta}}-E_{n \vartheta}=o_{p}(1)$ by Equation 4.10 . Hence

$$
\begin{aligned}
0= & E_{n \vartheta}+n^{-1 / 2} \sum_{i=1}^{n} E\left\{\delta w_{\hat{\vartheta}}(X)\left(Y-\hat{\vartheta}^{\top} X\right)\right\}+o_{p}(1) \\
= & n^{-1 / 2} \sum_{i=1}^{n}\left[\delta_{i} w_{\vartheta}\left(X_{i}\right)\left(Y_{i}-\vartheta^{\top} X_{i}\right)\right. \\
& \left.-E\left\{\delta w_{\vartheta}(X)\left(Y-\vartheta^{\top} X\right)\right\}+E\left\{\delta w_{\hat{\vartheta}}(X)\left(Y-\hat{\vartheta}^{\top} X\right)\right\}\right]+o_{p}(1) \\
= & n^{-1 / 2} \sum_{i=1}^{n} \delta_{i} w_{\vartheta}\left(X_{i}\right)\left(Y_{i}-\vartheta^{\top} X_{i}\right)-A n^{1 / 2}(\hat{\vartheta}-\vartheta) \\
& +n^{1 / 2} o(|\hat{\vartheta}-\vartheta|)+o_{p}(1)
\end{aligned}
$$

In the last equation we used Equation 4.8. Since the matrix $A$ is invertible by Assumption IV.2 we have proved the desired statement.

Refer to Schick (1996c) for more details on weighted least squares estimation. By Theorem 4.11 the influence function for weighted least squares is

$$
b_{i}=E\left\{\delta w_{\vartheta}(X) X^{\top}\right\}^{-1} \delta_{i} w_{\vartheta}\left(X_{i}\right)\left(Y_{i}-\vartheta^{\top} X_{i}\right)
$$

For this influence function

$$
\begin{aligned}
E\left(X b^{\top}\right) & =0 \\
E\left(\delta X b^{\top}\right) & =0 \\
E\left(b b^{\top}\right) & =E\left[E\left\{\delta w_{\vartheta}(X) X^{\top}\right\}^{-1} \delta w_{\vartheta}(X) \epsilon \epsilon w_{\vartheta}(X)^{\top} E\left\{\delta X w_{\vartheta}(X)^{\top}\right\}^{-1}\right] \\
& =\sigma^{2} E\left\{\delta w_{\vartheta}(X) X^{\top}\right\}^{-1} E\left\{\delta w_{\vartheta}(X) w_{\vartheta}(X)^{\top}\right\} E\left\{\delta X w_{\vartheta}(X)^{\top}\right\}^{-1} \\
E(b \delta \epsilon) & =E\left[E\left\{\delta w_{\vartheta}(X) X^{\top}\right\}^{-1} \delta w_{\vartheta}(X) \epsilon^{2}\right] \\
& =\sigma^{2} E\left\{\delta w_{\vartheta}(X) X^{\top}\right\}^{-1} E\left\{\delta w_{\vartheta}(X)\right\} .
\end{aligned}
$$


By Theorem III.3 the asymptotic variance for partial imputation using weighted least squares is

$$
\begin{gathered}
A V_{P I_{-} W L S}=\vartheta^{\top}\left\{E\left(X X^{\top}\right)-E(X) E(X)^{\top}\right\} \vartheta+\sigma^{2} E(\delta) \\
+2 \sigma^{2} E\left\{\delta w_{\vartheta}(X)\right\}^{\top} E\left\{\delta X w_{\vartheta}(X)^{\top}\right\}^{-1}\{E(X)-E(\delta X)\} \\
+\sigma^{2}\{E(X)-E(\delta X)\}^{\top} E\left\{\delta w_{\vartheta}(X) X^{\top}\right\}^{-1} E\left\{\delta w_{\vartheta}(X) w_{\vartheta}(X)^{\top}\right\} \\
E\left\{\delta X w_{\vartheta}(X)^{\top}\right\}^{-1}\{E(X)-E(\delta X)\} .
\end{gathered}
$$

ByTheorem III.5 the asymptotic variance for full imputation using weighted least squares is

$$
\begin{gathered}
A V_{F I_{-} W L S=}=\vartheta^{\top}\left\{E\left(X X^{\top}\right)-E(X) E(X)^{\top}\right\} \vartheta \\
+\sigma^{2} E(X)^{\top} E\left\{\delta w_{\vartheta}(X) X^{\top}\right\}^{-1} E\left\{\delta w_{\vartheta}(X) w_{\vartheta}(X)^{\top}\right\} \\
E\left\{\delta X w_{\vartheta}(X)^{\top}\right\}^{-1} E(X) .
\end{gathered}
$$

The two methods can be compared by determining where the difference between Equation 4.12 and Equation 4.13 is positive or negative. In other words, if

$$
\begin{aligned}
& A V_{P I_{-} W L S}-A V_{F I_{-} W L S} \\
= & \sigma^{2} E(\delta)+2 \sigma^{2} E\left\{\delta w_{\vartheta}(X)\right\}^{\top} E\left\{\delta X w_{\vartheta}(X)^{\top}\right\}^{-1}\{E(X)-E(\delta X)\} \\
& -2 \sigma^{2} E(\delta X)^{\top} E\left\{\delta w_{\vartheta}(X) X^{\top}\right\}^{-1} E\left\{\delta w_{\vartheta}(X) w_{\vartheta}(X)^{\top}\right\} E\left\{\delta X w_{\vartheta}(X)^{\top}\right\}^{-1} E(X) \\
& +\sigma^{2} E(\delta X)^{\top} E\left\{\delta w_{\vartheta}(X) X^{\top}\right\}^{-1} \\
& E\left\{\delta w_{\vartheta}(X) w_{\vartheta}(X)^{\top}\right\} E\left\{\delta X w_{\vartheta}(X)^{\top}\right\}^{-1} E(\delta X)
\end{aligned}
$$

is positive then partial imputation will have a larger asymptotic variance, implying the full imputation is better for that type of weighted least squares estimator. Whether Equation 4.14 is positive or negative depends on the type of weights chosen, 
as illustrated by examples in the following sections. Subsection 1 discusses ordinary least squares; other weighted least squares estimators are explored in Subsection 2 and Subsection 3 .

1. Ordinary least squares estimate of $\vartheta$

If OLS is used for the estimate for $\vartheta$, then $w_{\vartheta}(X)=X$, so by Equation 4.12

$$
\begin{aligned}
A V_{P I_{-} O L S}= & \vartheta\left\{E\left(X X^{\top}\right)-E(X) E(X)^{\top}\right\} \vartheta+\sigma^{2} E(\delta) \\
& +\sigma^{2} E(X)^{\top} E\left(\delta X X^{\top}\right)^{-1} E(X)-\sigma^{2} E(\delta X)^{\top} E\left(\delta X X^{\top}\right)^{-1} E(\delta X(41.15)
\end{aligned}
$$

By Equation 4.13 The asymptotic variance for full imputation using OLS with the influence function as shown for the partially imputed estimator is

$$
A V_{F I_{-} O L S}=\vartheta^{\top}\left\{E\left(X X^{\top}\right)-E(X) E(X)^{\top}\right\} \vartheta+\sigma^{2} E(X)^{\top} E\left(\delta X X^{\top}\right)^{-1} E(X)
$$

The difference between the fully imputed estimator and the partially imputed estimator with OLS is

$$
A V_{P I_{-} O L S}-A V_{F I_{-} O L S}=\sigma^{2}\left\{E(\delta)-E(\delta X)^{\top} E\left(\delta X X^{\top}\right)^{-1} E(\delta X)\right\} .
$$

This is nonnegative by the Cauchy Swarz inequality, so partial imputation will have at least as much asymptotic variance as full imputation using Ordinary Least Squares. In the case where $\epsilon$ is normally distributed the OLS estimator will match the efficient estimator. To see this note that under normality $\mathbb{I}=1 / \sigma^{2}$, which means from Equation 4.2 ,

$$
E\left(\delta \zeta \zeta^{\top}\right)=\frac{1}{\sigma^{2}} E\left(\delta X X^{\top}\right) .
$$


Then the asymptotic variance for partial imputation with an efficient estimator comes from Equation 4.3

$$
\begin{aligned}
A V_{P I-E F F}= & \vartheta^{\top}\left\{E\left(X X^{\top}\right)-E(X) E(X)^{\top}\right\} \vartheta+\sigma^{2} E(\delta) \\
& +\sigma^{2}\{E(X)+E(\delta X)\}^{\top} E\left(\delta X X^{\top}\right)^{-1}\{E(X)-E(\delta X)\} .
\end{aligned}
$$

This matches the partial imputation method with an OLS estimator given in Equation 4.15. For full imputation the efficient estimator when $\mathbb{I}=1 / \sigma^{2}$ is

$$
A V_{F I_{-} E F F}=\vartheta^{\top}\left\{E\left(X X^{\top}\right)-E(X) E(X)^{\top}\right\} \vartheta+E(\delta X)^{\top} E\left(\delta X X^{\top}\right)^{-1} E(\delta X) .
$$

which matches asymptotic variance for the full imputation with an OLS estimator given in Equation 4.16. This shows the approach using the OLS method is asymptotically equivalent to the approach using the efficient method. Consider the case when the error distribution is unknown. We will compare the asymptotic variance of the fully imputed estimator based on the OLS and the fully imputed estimator that uses an efficient estimator. By Equations 4.16 and 4.4 the difference of the asymptotic variances is

$$
\begin{aligned}
& A V_{F I_{-} O L S}-A V_{F I_{-} E F F} \\
= & \left\{\sigma^{2} E(X)^{\top} E\left(\delta X X^{\top}\right)^{-1} E(X)\right\}-\left\{E(X)^{\top} E\left(\delta \zeta \zeta^{\top}\right)^{-1} E(X)\right\} .
\end{aligned}
$$

Using the notation for $Q_{0}$ given in Equation 4.6 and for $C s$ given in Equation 4.5 this can be written as

$$
\begin{aligned}
& A V_{F I_{-} O L S}-A V_{F I_{-} E F F} \\
= & \left(\sigma^{2} \mathbb{I}-1\right) \sigma^{2} Q_{0} C_{0}\left(\sigma^{2} \mathbb{I} C_{0}+Q_{0}\right)^{-1} .
\end{aligned}
$$


By the Cauchy Schwarz inequality $C_{0}$ is positive, as is the quadratic form $Q_{0}$, so this difference is nonnegative when $\mathbb{I} \geq 1 / \sigma^{2}$. This shows that the fully imputed estimator using OLS has in general larger asymptotic variance than the corresponding estimator with an efficient estimator of $\vartheta$. This holds if the usual regularity conditions are met. This will not hold for the uniform distribution, for example, where the support for $\epsilon$ depends on the parameter.

\section{Constant weight for WLS}

If a constant weight, say $w_{\vartheta}(X)=1$ is used, then the difference between partial and full imputation given in Equation 4.14 becomes

$$
\begin{aligned}
& A V_{P I_{-} W L S}-A V_{F I_{-} W L S} \\
= & \sigma^{2} E(\delta)+2 \sigma^{2} E(\delta) E(\delta X)^{-1} E(X)-2 \sigma^{2} E(\delta)-2 \sigma^{2} E(\delta) E(\delta X)^{-1} E(X)+\sigma^{2} E(\delta) \\
= & 0 .
\end{aligned}
$$

This implies that full imputation and partial imputation are asymptotically equivalent if the weights are constant. This can be seen by rewriting the weighted estimating equation in Equation 4.7 .

$$
\begin{aligned}
\sum_{i=1}^{n} \delta_{i}\left(Y_{i}-\hat{\vartheta}_{w=1}^{\top} X_{i}\right) & =0 \\
\sum_{i=1}^{n} \delta_{i} Y_{i} & =\sum_{i=1}^{n} \delta_{i} \hat{\vartheta}_{w=1}^{\top} X_{i} \\
\hat{\vartheta}_{w=1} & =\sum_{i=1}^{n}\left(\delta_{i} Y_{i}\right) \sum_{i=1}^{n}\left(\delta_{i} X_{i}^{\top}\right)\left\{\sum_{i=1}^{n}\left(\delta_{i} X_{i}\right) \sum_{i=1}^{n}\left(\delta_{i} X_{i}^{\top}\right)\right\}^{-1} .
\end{aligned}
$$

In one dimension this is easier to see the implication of using this weight where $\hat{\vartheta}$ is

$$
\hat{\vartheta}_{w=1}=\frac{\sum_{i=1}^{n} \delta_{i} Y_{i}}{\sum_{i=1}^{n} \delta_{i} X_{i}} .
$$


Returning to Equation 3.8, which defines partial imputation,

$$
\begin{aligned}
\widehat{E(Y)_{P I}} & =\frac{1}{n} \sum_{i=1}^{n}\left\{\delta_{i} Y_{i}+\left(1-\delta_{i}\right) \hat{\vartheta}_{w=1}^{\top} X_{i}\right\} \\
& =\frac{1}{n} \sum_{i=1}^{n}\left(\delta_{i} Y_{i}\right)+\frac{1}{n} \sum_{i=1}^{n}\left(\hat{\vartheta}_{w=1}^{\top} X_{i}\right)-\frac{1}{n} \sum\left(\delta_{i} \hat{\vartheta}_{w=1}^{\top} X_{i}\right) .
\end{aligned}
$$

Now using Equation 4.17 in the middle,

$$
\begin{aligned}
\widehat{E(Y)_{P I}} & =\frac{1}{n} \sum_{i=1}^{n}\left(\delta_{i} Y_{i}\right)+\frac{1}{n} \sum_{i=1}^{n}\left(\hat{\vartheta}_{w=1}^{\top} X_{i}\right)-\frac{1}{n} \sum\left(\delta_{i} Y_{i}\right) \\
& =\frac{1}{n} \sum_{i=1}^{n} \hat{\vartheta}_{w=1}^{\top} X_{i},
\end{aligned}
$$

which equals the fully imputed estimator in Equation 3.18 with the estimator having weights equal to one. The proof for any constant weight is similar. Partial imputation is the same as full imputation when a constant weight is used.

\section{A poor choice of weights in WLS}

Full imputation depends heavily on the estimate for $\vartheta$, so if weighted least squares is used with weights that are chosen poorly enough full imputation will have more asymptotic variance than partial imputation. One example of this is

$$
w_{\vartheta}(X)=\frac{X-E(X)}{E(\delta \mid X)} .
$$


In this case

$$
\begin{aligned}
E\left(\delta w_{\vartheta}(X)\right) & =E\left\{\delta \frac{X-E(X)}{E(\delta \mid X)}\right\} \\
& =E\left[E\left\{\delta \frac{X-E(X)}{E(\delta \mid X)} \mid X\right\}\right] \\
& =E\left\{\frac{X-E(X)}{E(\delta \mid X)} E(\delta \mid X)\right\} \\
& =E(X)-E(X) \\
& =0
\end{aligned}
$$

as well as

$$
\begin{aligned}
E\left(\delta X w_{\vartheta}(X)^{\top}\right) & =E\left\{\frac{X X^{\top}-X E(X)^{\top}}{E(\delta \mid X)} E(\delta \mid X)\right\} \\
& =E\left(X X^{\top}\right)-E(X) E(X)^{\top},
\end{aligned}
$$

and

$$
\begin{aligned}
E\left(\delta w_{\vartheta}(X) w_{\vartheta}(X)^{\top}\right) & =E\left[\frac{\{X-E(X)\}\{X-E(X)\}^{\top}}{E(\delta \mid X)} E(\delta \mid X)\right] \\
& =E\left\{X X^{\top}-2 X E(X)^{\top}+E(X) E(X)^{\top}\right\} \\
& =E\left(X X^{\top}\right)-E(X) E(X)^{\top} .
\end{aligned}
$$

The difference in asymptotic variance between partial and full imputation using Equation 4.14 is

$$
\begin{aligned}
& A V_{P I_{-} W L S}-A V_{F I_{-} W L S} \\
= & \sigma^{2} E(\delta)-2 \sigma^{2} E(\delta X)^{\top}\left\{E\left(X X^{\top}\right)-E(X) E(X)^{\top}\right\}^{-1} E(X) \\
& +\sigma^{2} E(\delta X)^{\top}\left\{E\left(X X^{\top}\right)-E(X) E(X)^{\top}\right\}^{-1} E(\delta X) \\
= & \sigma^{2} E(\delta)+\sigma^{2} E(\delta X)^{\top}\left\{E\left(X X^{\top}\right)-E(X) E(X)^{\top}\right\}^{-1}\{E(\delta X)-2 E(X)\}(.4 .1
\end{aligned}
$$

When the difference is negative partial imputation has less variance than full impu- 
tation. The following explanation shows that this scenario is possible. The method is to increase the value of Equation 4.18 , but then show a scenario where the larger value is still negative. That implies Equation 4.18 would be negative and partial imputation is better than full imputation in that case. Consider the inequality

$$
E(\delta X)\{\operatorname{Cov}(X)\}^{-1} E(\delta X) \leq E(\delta X)\{\operatorname{Cov}(X)\}^{-1} E(X)
$$

When $X$ is positive Equation 4.19 is true. When $X$ is negative then the two expected values in each side of the equation net a postive result, so Equation 4.19 is still true. This means Equation 4.18 can be increased by

$$
\begin{aligned}
& \sigma^{2} E(\delta)+\sigma^{2} E(\delta X)^{\top}\left\{E\left(X X^{\top}\right)-E(X) E(X)^{\top}\right\}^{-1}\{E(\delta X)-2 E(X)\} \\
\leq & \sigma^{2} E(\delta)-\sigma^{2} E(\delta X)^{\top}\left\{E\left(X X^{\top}\right)-E(X) E(X)^{\top}\right\}^{-1} E(\delta X) .
\end{aligned}
$$

If the larger equation is still negative, then partial imputation has less variability than full imputation. In other words, if

$$
0 \geq E(\delta)-E(\delta X)^{\top}\left\{E\left(X X^{\top}\right)-E(X) E(X)^{\top}\right\}^{-1} E(\delta X)
$$

In a one dimensional case this is means partial imputation has less variability than full imputation if

$$
\operatorname{var}(X) \leq \frac{E^{2}(\delta X)}{E(\delta)} .
$$

To see that this scenario is possible consider the case when the missing structure is symmetric over a symmetric covariate. This assumption as shown in Theorem III.1 implies

$$
E(\delta X)=E(\delta) E(X) .
$$


Then Equation 4.20 becomes

$$
E\left(X^{2}\right) \leq\{E(\delta)+1\} E^{2}(X) .
$$

Such a scenario is explored in Section V. In that case the estimate of $\vartheta$ has so much variability that full imputation has more asymptotic variance than partial imputation. 


\section{CHAPTER V}

\section{EXAMPLES}

In this section the goal is to estimate $E(Y)$ under the simple linear model where $Y=\vartheta X+\epsilon$. Let $X \sim \operatorname{Uniform}(0,2)$, and $\vartheta=3$. The following error distributions of $\epsilon$ are considered:

1. $\epsilon \sim \operatorname{Uniform}(-1,1)$ which breaks the regularity conditions;

2. $\epsilon \sim \operatorname{Normal}(0,1)$ where the OLS method is efficient;

3. $\epsilon \sim$ Double Exponential with a mean of 0 and variance 2;

4. $\epsilon \sim t$ with 3 degrees of freedom;

5. $\epsilon \sim$ Gamma with a variance of 2 shifted to have a mean of 0 .

6. $\epsilon \sim$ Logistic. The standard Logistic distribution has heavy tails compared to the Normal distribution.

7. $\epsilon \sim$ Gumbel. The standard Gumbel distribution is similar to the Normal but is skewed.

Table[lists some calculations that are helpful for finding the asymptotic variance under each error distribution.

For each scenario the asymptotic variances will be compared using the following methods:

1. LD - Listwise Deletion (Equation 3.5);

2. PS - Propensity Score using the true $\pi(X)$ (Equation 3.7);

3. PI_EFF - Partial Imputation with an efficient estimate (Equation 4.3); 
Table I. The variance and Fisher's information for various error distributions.

\begin{tabular}{|c|c|c|}
\hline & $\sigma^{2}$ & $\mathbb{I}$ \\
\hline $\mathrm{U}(-1,1)$ & .333 & 0 \\
\hline $\mathrm{N}(0,1)$ & 1 & 1 \\
\hline$t_{3}$ & 0.6667 & 3 \\
\hline Gamma(2,1)-2 & 30.474 & 2 \\
\hline Logistic & 0.3333 & 3.2899 \\
\hline Gumbel & 1 & 1.64493 \\
\hline DExp & 2 & 1 \\
\hline
\end{tabular}

4. FI_EFF - Full imputation with an efficient estimate (Equation 4.4);

5. PI_OLS - Partial Imputation using Ordinary Least Squares (Equation 4.15);

6. FI_OLS - Full Imputation using Ordinary Least Squares (Equation 4.16).

A. Symmetric missing structure

Consider the case where the missing structure $\pi(X)=E(\delta \mid X)$ has the piecewise form

$$
\pi(X)= \begin{cases}.2 & 0<X<.5 \\ .8 & .5 \leq X<1.5 \\ .2 & 1.5 \leq X \leq 2\end{cases}
$$

which means $\delta$ is 1 with probability of 0.20 on the ends of $X$, and is 1 with probability 0.80 in the middle range of $X$. Figure 3 shows a plot of $\pi(X)$. 


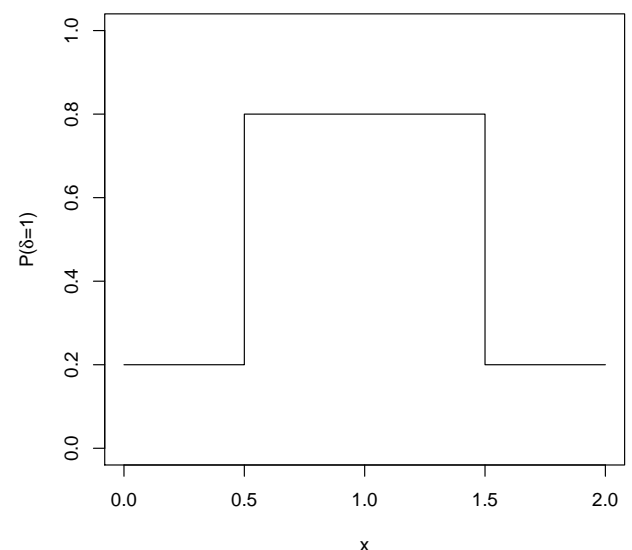

Fig. 3. Symmetric missing structure. The missingness is centered over $E(X)=1$ and is stepwise.

It is easy to show that $E(X)=1, E\left(X^{2}\right)=4 / 3$, and

$$
E(\delta)=(0.2)(1 / 4)+(0.8)(1 / 2)+(0.2)(1 / 4)=1 / 2
$$

The following expectation is also needed:

$$
\begin{aligned}
E(\delta X) & =\int_{0}^{1} \int_{0}^{2} \delta x G(d x) B_{\pi(x)}(d \delta) \\
& =(0.2) \int_{0}^{0.5} x \frac{1}{2} d x+(0.8) \int_{0.5}^{1.5} x \frac{1}{2} d x+(0.2) \int_{1.5}^{2} x \frac{1}{2} d x \\
& =0.5
\end{aligned}
$$

And by using the same method $E\left(\delta X^{\top} X\right)=71 / 120 \approx 0.59167$. The next expectation uses the fact that $E(\delta \mid X)=(0.2) 1(0 \leq X \leq 0.5)+(0.8) 1(0.5<X \leq 1.5)+$ $(0.2) 1(1.5<X \leq 2)$. This means

$$
\begin{aligned}
E\left\{\frac{1}{E(\delta \mid X)}\right\} & =\int_{0}^{0.5} \frac{1}{0.2} \frac{1}{2} d x+\int_{0.5}^{1.5} \frac{1}{0.8} \frac{1}{2} d x+\int_{1.5}^{2} \frac{1}{0.2} \frac{1}{2} d x \\
& =\frac{25}{8}=3.125
\end{aligned}
$$


Also,

$$
\begin{aligned}
E\left\{\frac{X^{2}}{E(\delta \mid X)}\right\} & =\int_{0}^{0.5} \frac{1}{0.2} x^{2} \frac{1}{2} d x+\int_{0.5}^{1.5} \frac{1}{0.8} x^{2} \frac{1}{2} d x+\int_{1.5}^{2} \frac{1}{0.2} x^{2} \frac{1}{2} d x \\
& =\frac{445}{96} \approx 4.635417 .
\end{aligned}
$$

This specific model is interesting because $E(\delta X)=E(\delta) E(X)$. See Theorem III.1 for an explanation of how this implies the listwise deletion method is not biased. The asymptotic variances are given in Table II. It is easy to see that the Propensity Score method which uses the true $\pi(X)$ has the highest variance. The Listwise Deletion method has less variance than the propensity score method because the error structure is symmetric over $E(X)$. When the error structure is uniform the regularity conditions do not hold, and there is no regular efficient estimator for the mean response. In this case the estimates using an OLS estimate for $\vartheta$ have the least variance. When the errors are normal the imputation methods to estimate the mean response have the same asymptotic variance whether an OLS estimate or the efficient estimate of $\vartheta$ is used. See Subsection 1 for discussion on how the OLS estimate is efficient under normality. In every other case using full imputation with the efficient estimate of $\vartheta$ results in the smallest asymptotic variance.

Now consider weighted least squares where the choice of weights follows Subsection 3 ,

$$
w_{\vartheta}(X)=\frac{X-E(X)}{\pi(X)}
$$

The asymptotic variances for partial and full imputation when the errors are standard normally distributed are

$$
\begin{aligned}
& A V_{P I_{-W L S b a d}}=997.5 \\
& A V_{\text {FI_WLSbad }}=998.0
\end{aligned}
$$


Table II. The asymptotic variances where the missing structure is symmetric.

\begin{tabular}{|c|c|c|c|c|c|c|}
\hline & LD & PS & PI_OLS & PI_EFF & FI_OLS & FI_EFF \\
\hline $\mathrm{U}(-1,1)$ & 13.0 & 33.8 & 3.59 & 3.67 & 3.56 & 3.67 \\
\hline $\mathrm{N}(0,1)$ & 14.3 & 35.8 & 4.77 & 4.77 & 4.69 & 4.69 \\
\hline Dexp & 16.3 & 39.0 & 6.54 & 6.20 & 6.38 & 5.23 \\
\hline$t_{3}$ & 18.3 & 42.1 & 8.30 & 7.79 & 8.07 & 7.39 \\
\hline Gamma(2,1)-2 & 16.3 & 39.0 & 6.54 & 6.20 & 6.38 & 5.93 \\
\hline Logistic & 18.9 & 43.0 & 8.82 & 8.75 & 8.56 & 8.48 \\
\hline Gumbel & 15.6 & 37.9 & 5.91 & 5.72 & 5.78 & 5.53 \\
\hline
\end{tabular}

As discussed in Subsection 3, this version of weighted least squares causes full imputation to have more asymptotic variance than partial imputation. From Equation 4.20 it can be seen that this difference would be even more drastic if $\pi(X)$ was closer to zero or if the variance of $X$ was smaller.

B. Gaussian missing structure

Now consider a Gaussian missing structure where

$$
\pi(X a)=E(\delta \mid X)=\frac{1}{\sqrt{2 \pi}} e^{-\frac{\left(X-\mu_{X}\right)^{2}}{\sigma_{X}}} .
$$

This model is similar to the symmetric missing structure discussed earlier, but it is a smooth function. The plot for the Gaussian missing structure is given in Figure 4 .

As before $E(X)=1, E\left(X^{2}\right)=4 / 3$, and $\sigma_{X}=1 / 3$. The other needed pieces are 


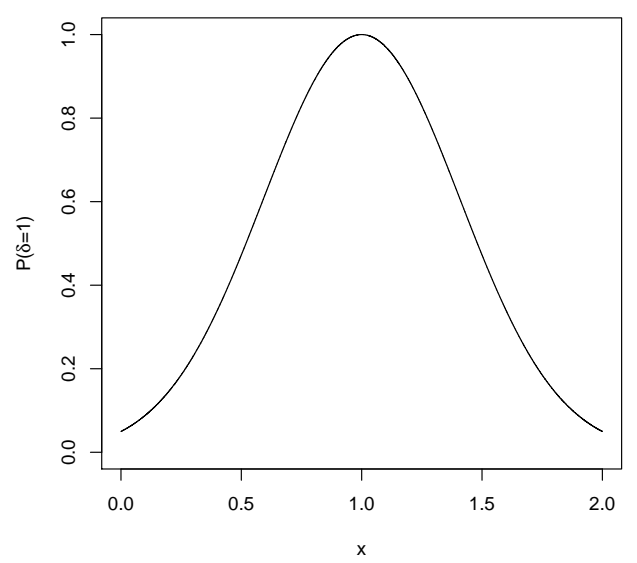

Fig. 4. Gaussian missing structure.

solved as

$$
\begin{aligned}
E(\delta) & =\int_{0}^{2} \frac{1}{2} e^{-3(X-1)^{2}} d X=0.5043435 \\
E(\delta X) & =\int_{0}^{2} X \frac{1}{2} e^{-3(X-1)^{2}} d X=0.5043435 \\
E\left(\delta X^{2}\right) & =\int_{0}^{2} X^{2} \frac{1}{2} e^{-3(X-1)^{2}} d X=0.5801029 \\
E\left\{\frac{1}{E(\delta \mid X)}\right\} & =\int_{0}^{2} \frac{1}{2} e^{3(X-1)^{2}} d X=4.2222 \\
E\left\{\frac{X^{2}}{E(\delta \mid X)}\right\} & =\int_{0}^{2} \frac{1}{2} X^{2} e^{3(X-1)^{2}} d X=6.8661
\end{aligned}
$$

The asymptotic variances for this model are given in Table III. The results are similar to the symmetric model. The uniform distribution shows a smaller asymptotic variance for methods that use the OLS estimator for $\vartheta$, and under the normal distribution the OLS and efficient estimates for $\vartheta$ agree. For every other error distribution the fully imputed estimator with an efficient estimator of $\vartheta$ has the smallest asymptotic variance. 
Table III. The asymptotic variances where the missing structure is Gaussian.

\begin{tabular}{|c|c|c|c|c|c|c|}
\hline & LD & PS & PI_EFF & FI_EFF & PI_OLS & FI_OLS \\
\hline U(-1,3) & 12.1 & 54.2 & 3.661 & 3.661 & 3.597 & 3.575 \\
\hline Normal & 13.5 & 57.0 & 4.79 & 4.724 & 4.79 & 4.724 \\
\hline DExp & 15.5 & 61.2 & 6.282 & 6.049 & 6.579 & 6.448 \\
\hline$t_{3}$ & 17.5 & 65.5 & 7.924 & 7.574 & 8.369 & 8.171 \\
\hline Gamma(2,1)-2 & 15.5 & 61.2 & 6.282 & 6.049 & 6.579 & 6.448 \\
\hline Logistic & 18.0 & 66.7 & 8.84 & 8.60 & 8.89 & 8.67 \\
\hline Gumbel & 14.8 & 59.7 & 5.78 & 5.62 & 5.94 & 5.84 \\
\hline
\end{tabular}

As in the previous section consider the weighted least squares where the choice of weights follows Subsection 3. For this missing structure the assumption of $E(\delta X)=$ $E(\delta) E(X)$ does not hold. The asymptotic variances for partial and full imputation when the errors are standard normally distributed are

$$
\begin{aligned}
& A V_{P I_{-} W L \text { Sbad }}=997.5 \\
& A V_{F I_{-} W L S b a d}=998.0
\end{aligned}
$$

Again this shows an example where full imputation has more asymptotic variance than partial imputation due to the poor estimation of the parameter $\vartheta$.

\section{Exponential missing structure}

Now consider an exponential missing structure where

$$
\pi(X)=E(\delta \mid X)=\left(1+\exp \left\{\frac{X-\mu_{X}}{\sigma_{X}}\right\}\right)^{-1} .
$$


The plot for the exponential missing structure is given in Figure 5 .

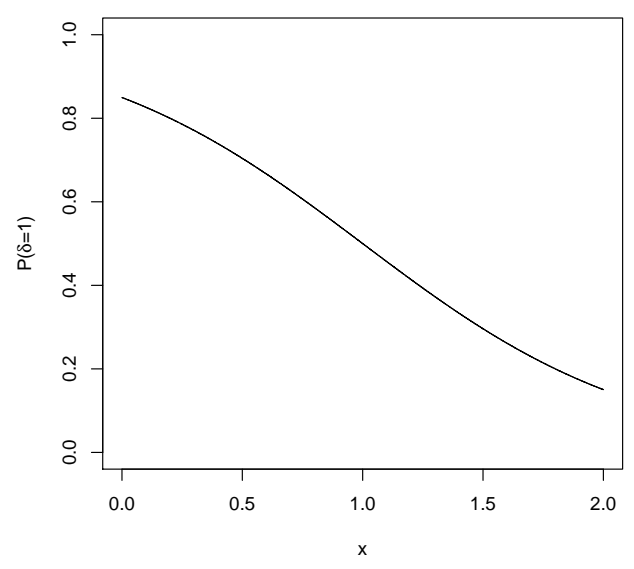

Fig. 5. Exponential missing structure.

As before $E(X)=1, E\left(X^{2}\right)=4 / 3$, and $\sigma_{X}=1 / 3$. The other needed pieces are solved as

$$
\begin{aligned}
E(\delta) & =\int_{0}^{2} \frac{1}{2} \frac{1}{1+e^{(X-1) \sqrt{3}}} d X=1 / 2 \\
E(\delta X) & =\int_{0}^{2} X \frac{1}{2} \frac{1}{1+e^{(X-1) \sqrt{3}}} d X=0.37355217 \\
E\left(\delta X^{2}\right) & =\int_{0}^{2} X^{2} \frac{1}{2} \frac{1}{1+e^{(X-1) \sqrt{3}}} d X=0.4137710 \\
E\left\{\frac{1}{E(\delta \mid X)}\right\} & =\int_{0}^{2} \frac{1}{2}\left\{1+e^{(X-1) \sqrt{3}}\right\} d X=2.58059 \\
E\left\{\frac{X^{2}}{E(\delta \mid X)}\right\} & =\int_{0}^{2} X^{2} \frac{1}{2}\left\{1+e^{(X-1) \sqrt{3}}\right\} d X=5.1455
\end{aligned}
$$

Here $E(X)=1, E\left(X^{2}\right)=4 / 3, \sigma_{X}=1 / 3, E(\delta)=1 / 2, E(\delta X)=0.37355$, $E\left(\delta X^{2}\right)=0.41377, E\{1 / E(\delta \mid X)\}=2.58059$, and $E\left\{X^{2} / E(\delta \mid X)\right\}=5.1455$. The asymptotic variances for this model are given in Table IV. For this scenario the Listwise Deletion method is not of interest because it is biased. Asymptotically the Listwise Deletion estimator will not converge to the true $E(Y)$. The Propensity Score 
method has much higher variance than the imputation methods. As in the previous two sections when the error structure is Uniform the OLS method outperforms the other methods. When the error structure is Normal the OLS and efficient estimates are the same. The best method in every case except the Uniform is full imputation with an efficient estimate of $\vartheta$.

Table IV. The asymptotic variances where the missing structure is exponential.

\begin{tabular}{|c|c|c|c|c|c|c|}
\hline & LD & PS & PI_EFF & FI_EFF & PI_OLS & FI_OLS \\
\hline U(-1,1) & - & 38.2 & 4.194 & 4.194 & 3.86 & 3.806 \\
\hline Normal & - & 39.9 & 5.58 & 5.417 & 5.58 & 5.417 \\
\hline DExp & - & 42.51 & 7.138 & 6.647 & 8.159 & 7.834 \\
\hline$t_{3}$ & - & 45.11 & 9.206 & 8.47 & 10.739 & 10.25 \\
\hline Gamma(2,1)-2 & - & 42.51 & 7.138 & 6.647 & 8.159 & 7.834 \\
\hline Logistic & - & 45.80 & 11.2 & 10.71 & 11.49 & 10.95 \\
\hline Gumbel & - & 41.55 & 6.65 & 6.29 & 7.24 & 6.98 \\
\hline
\end{tabular}

As in the previous two sections consider the weighted least squares where the choice of weights follows Subsection 3. For this missing structure the assumption of $E(\delta X)=E(\delta) E(X)$ does not hold. The asymptotic variances for partial and full imputation when the errors are standard normally distributed are

$$
\begin{aligned}
& A V_{P I_{-} W L S b a d}=997.7 \\
& A V_{F I \_W L S b a d}=998.0
\end{aligned}
$$

Again this shows an example where full imputation has more asymptotic variance than partial imputation due to the poor estimation of the parameter $\vartheta$. 
D. Simulation results with finite sample sizes

This section uses simulations to compare Full Imputation, Partial Imputation, and the Propensity Score method. The simulated Mean Square Error of the estimators for $E(Y)$ is shown for various estimates of the parameter $\vartheta$. OLS denotes the Ordinary Least Squares estimate, OSI denotes to the One Step Improvement estimator, and MELE denotes the Maximum Empirical Likelihood Estimator with one, two or three constraints which are called MELE1, MELE2, and MELE3, respectively. The results are from 20,000 simulations.

Figure 6 shows results with no missing data when the errors are normally distributed. Because there is no missing data the Partial Imputation method is simply the empirical estimator $\bar{Y}$ and therefore does not depend on $\vartheta$. Since the errors are normal Full imputation is asymptotically efficient for all five estimates of $\vartheta$. For small sample sizes the OSI method has a larger MSE, partly due to the difficulty of estimating the score function. The MSE for the Propensity Score method is given below each plot, which is again the MSE of $\bar{Y}$. 


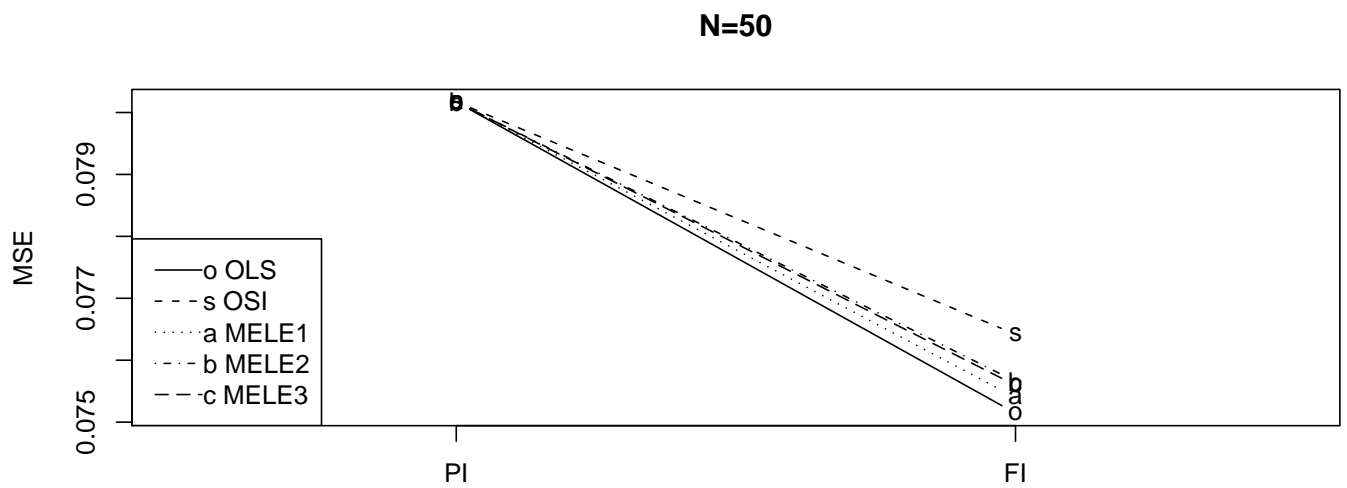

Propensity Score Method $=0.078944$

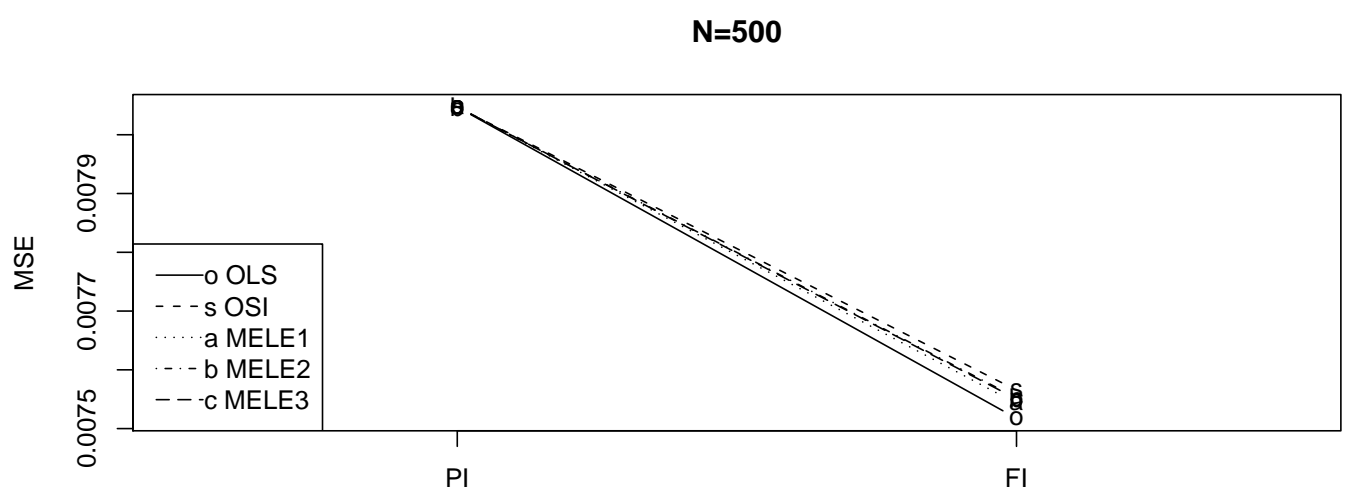

Propensity Score Method $=0.008003$

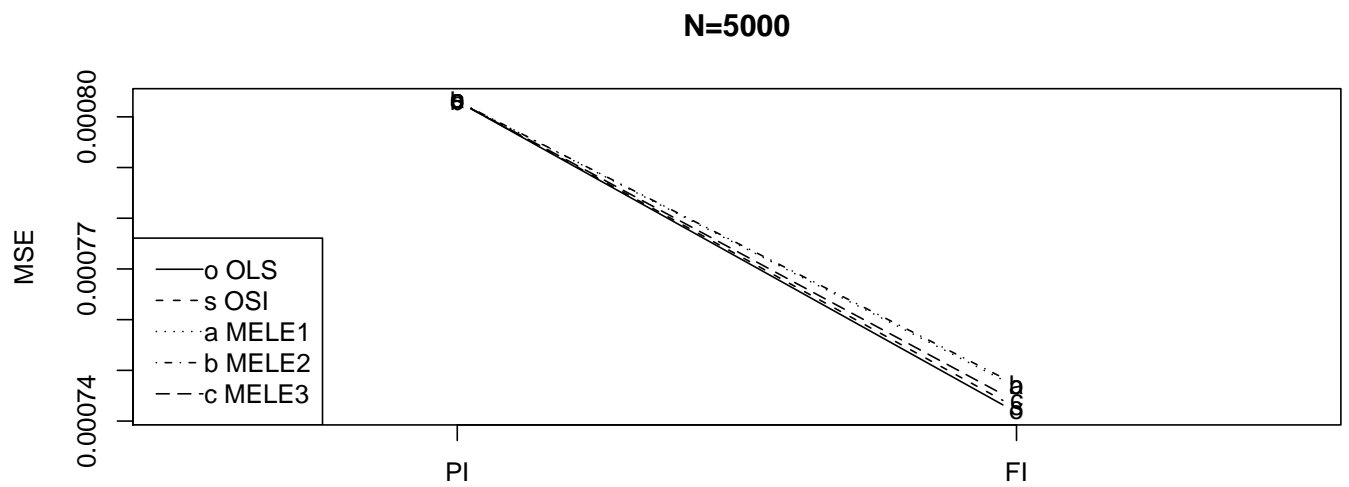

Propensity Score Method $=0.000815$

Fig. 6. MSE for estimating $E[Y]$ under normal errors with no missing data 


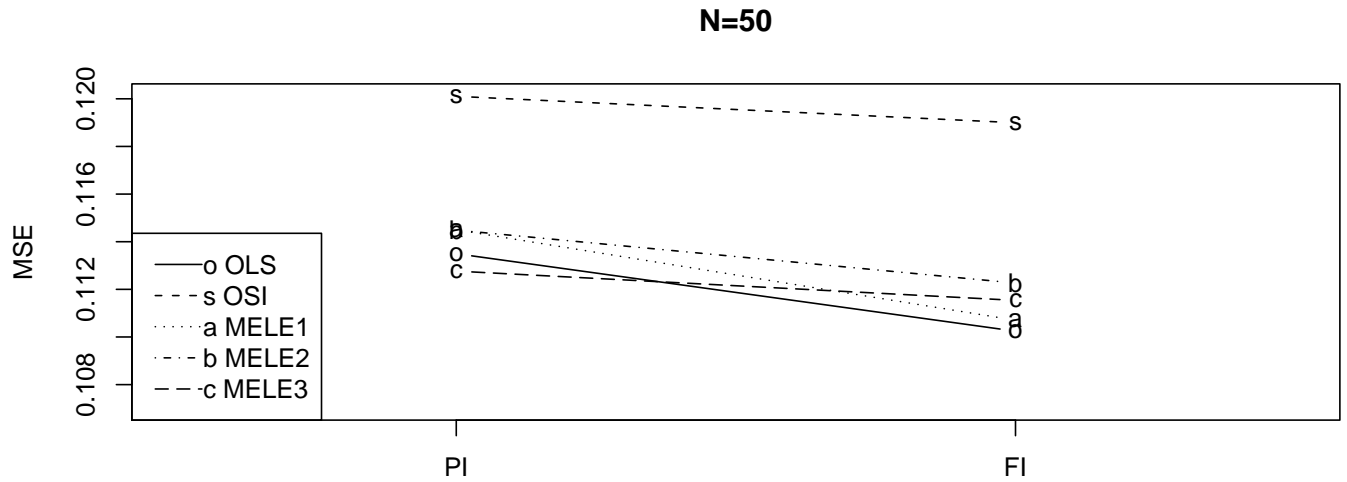

Propensity Score Method $=0.793352$

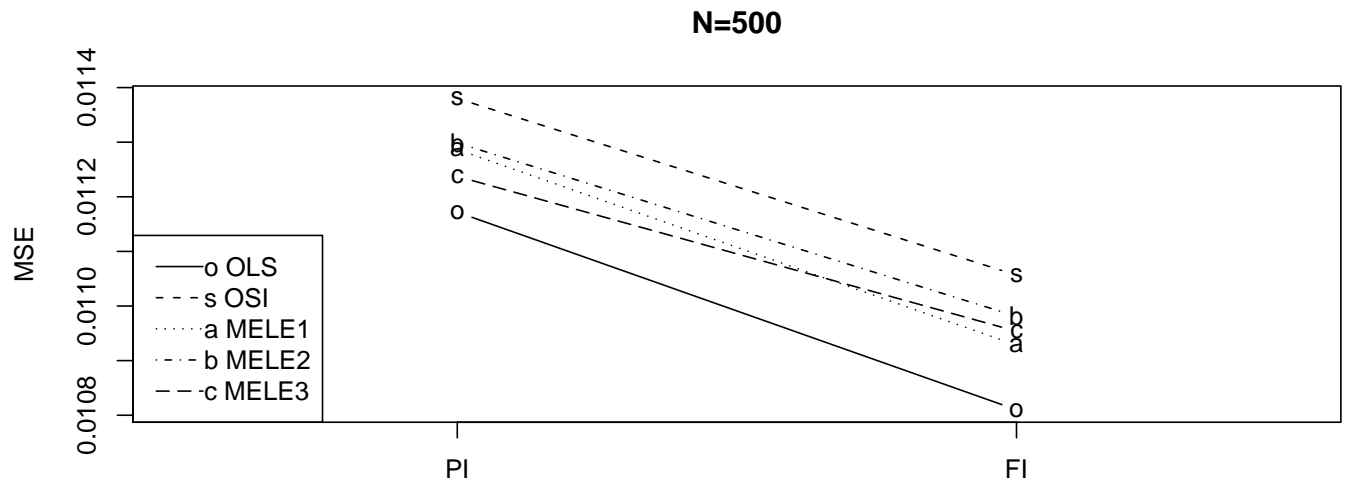

Propensity Score Method $=0.080086$

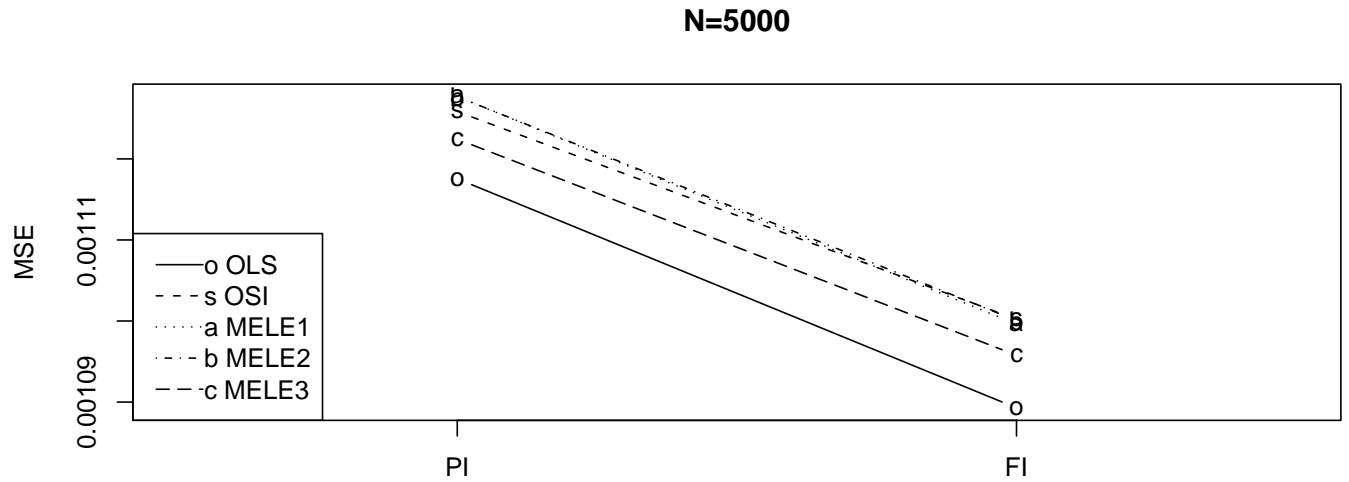

Propensity Score Method $=0.007892$

Fig. 7. MSE for estimating $E[Y]$ under normal errors with an exponential missing structure 
Figure 7 shows results from an exponential missing structure. With missing data the variance of the partial imputation method depends on the estimate of $\vartheta$. The errors are again generated from the normal distribution so Full Imputation is efficient with any estimate of $\vartheta$. With small sample sizes the OLS estimate has the smallest MSE and OSI has a larger MSE. The MELE3 method performs better than MELE1 or MELE2 for the larger sample size, which is expected. In every case Full Imputation is better than Partial Imputation.

Figure 8 shows results with gamma errors and an exponential missing stucture. In this case for small sample sizes there is a dramatic difference in the MSE for estimating $E(Y)$ depending on the estimate of $\vartheta$. The OSI estimate cannot estimate the score function well for small sample sizes, but for a large sample size it performs very well. The OLS estimate has more variance, and that difference grows as the sample size increases.

For graphs of other error distributions across the different structures of the missing data, as well as tables showing the MSE values see Appendix A. 


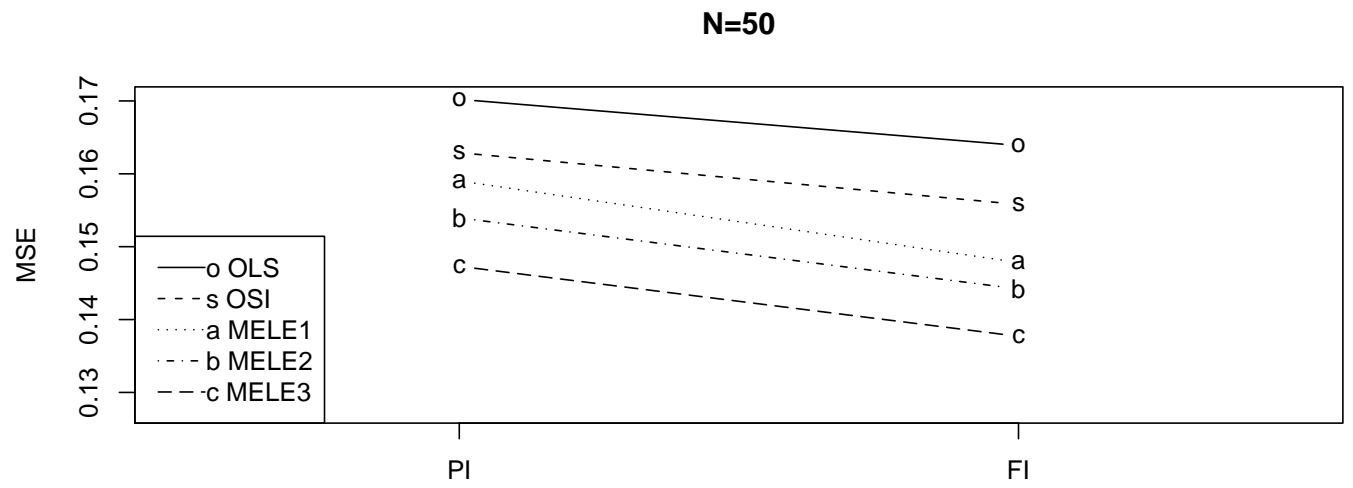

Propensity Score Method $=0.851671$

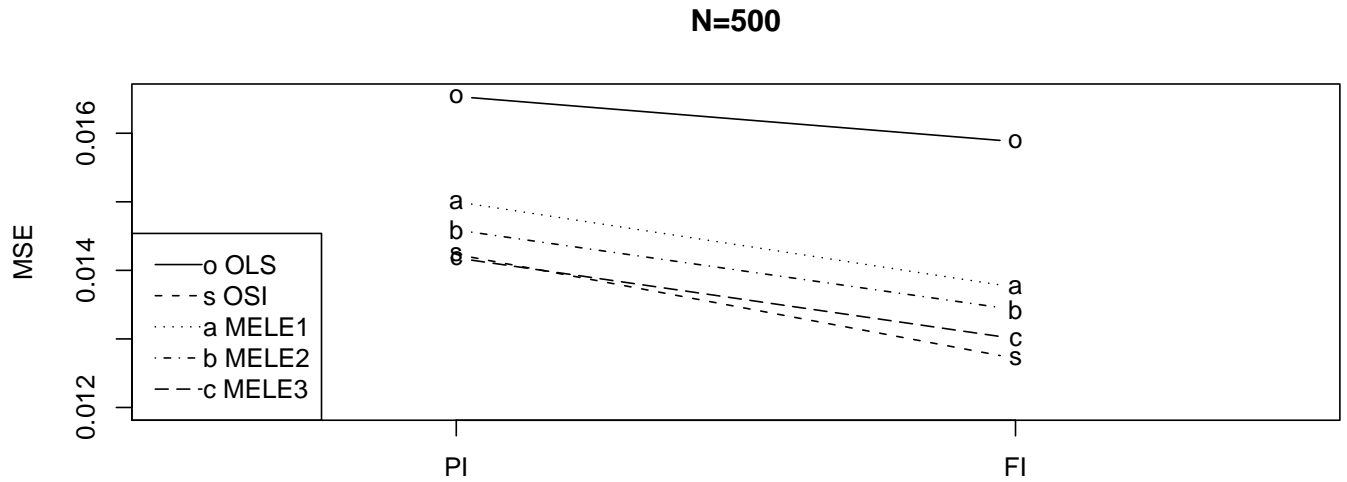

Propensity Score Method $=0.084997$

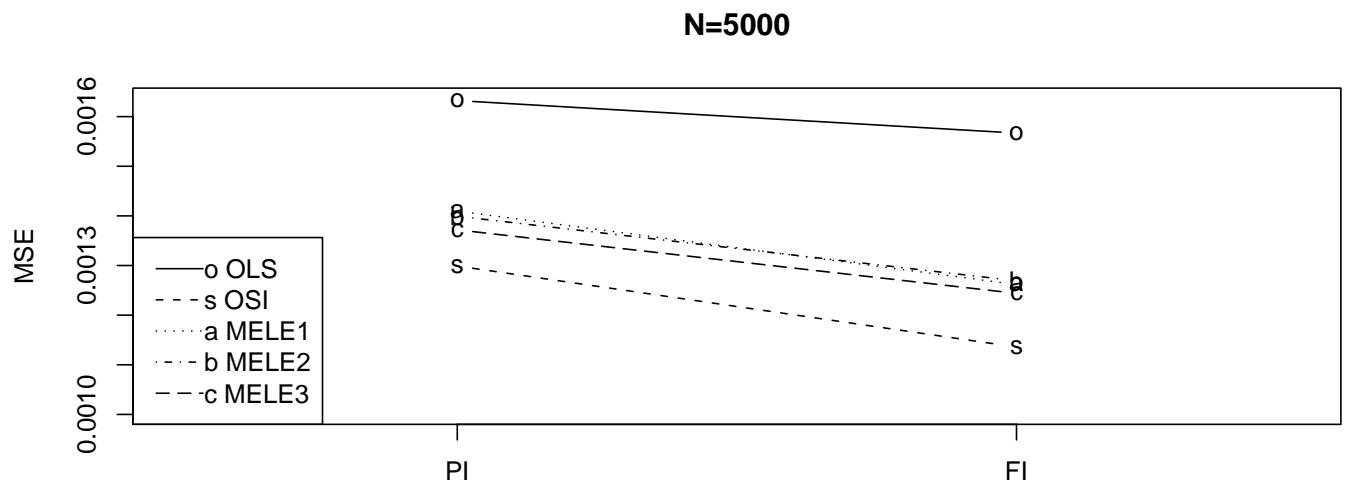

Propensity Score Method $=0.008569$

Fig. 8. MSE for estimating $E[Y]$ under gamma errors with an exponential missing structure 


\section{CHAPTER VI}

\section{SUMMARY}

When the unknown error distribution is in fact normal the Ordinary Least Squares estimator is efficient for estimating the parameter in linear regression. If the errors are not normal, then a One Step Improvement estimate or a Maximum Empirical Likelihood estimate can be used to estimate the parameter efficiently.

When estimating the mean response Listwise Deletion can be biased depending on the missing structure. The Propensity Score method is an improvement as it is unbiased, but neither Listwise Deletion nor the Propensity Score method are efficient. Estimating the mean response efficiently requires imputation. For both Partial Imputation and Full Imputation the performance depends on the estimate of the regression parameter, but in general Full Imputation is better than Partial Imputation. Only when the parameter is estimated very poorly will Partial Imputation have less variance than full imputation. The efficient estimate for the mean response is Full Imputation with an efficient estimate of the parameter. 


\section{REFERENCES}

Andrews, D. W. K. and D. Pollard (1994). An introduction to functional central limit theorems for dependent stochastic processes. International Statistical Review 62, 119-132. 49

Bell, B. A., J. D. Kromrey, and J. M. Ferron (2009). Missing data and complex samples: The impact of listwise deletion vs. subpopulation analysis on statistical bias and hypothesis test results when data are mcar and mar. Proceedings of the Joint Statistical Meetings, Survey Research Methods Section. 26

Dong, W. and X. C. Song (2009). Empirical likelihood for estimating equation with missing values. Annals of Statistics 37, 490-517. 25

Elliot, M. (2008). Healthy for life: Accounting for transcription errors using multiple imputation. CHANCE 21, 14-23. 1

Forrester, J., W. Hooper, H. Peng, and A. Schick (2003). On the construction of efficient estimators in semiparametric models. Statistical Decisions 21, 109-138. 2. 16

Koul, H. L., U. U. Müller, and A. Schick (2012). Complete case analysis revisited. Draft. For more information contact uschi@stat.tamu.edu. 17

Little, R. J. A. and D. B. Rubin (2002). Statistical Analysis with Missing Data (2 $2^{\text {nd }}$ ed.). Hoboken, NJ: Wiley-Interscience. 1

Müller, U. U. (2007). Weighted least squares estimators in possibly misspecified nonlinear regression. Metrika 66, 39-59. 47, 48 
Müller, U. U. (2009). Estimating linear functionals in nonlinear regression with responses missing at random. Ann. Stat. 37, 2245-2277. 1, 5, 40, 44, 48

Müller, U. U., A. Schick, and W. Wefelmeyer (2004). Estimating linear functionals of the error distribution in nonparametric regression. Journal of Statistical Planning and Inference 119, 75-93. 49

Müller, U. U., A. Schick, and W. Wefelmeyer (2006). Probability Statistics and Modeling in Public Health - Symposium in Honor of Marvin Zelen, pp. 350-363. New York, NY: Springer. 1, 4

Müller, U. U., A. Schick, and W. Wefelmeyer (2007). Estimating the error distribution function in semiparametric regression. Statistics $\&$ Decisions 25, 1-18. 158

Owen, A. B. (1988). Empirical likelihood ratio confidence intervals for a single functional. Biometrica 75, 237-249. 17

Owen, A. B. (2001). Empirical Likelihood. Boca Raton, FL: Chapman \& Hall/CRC. 18, 41

Peng, H. and A. Schick (2012). An empirical likelihood approach to goodness of fit testing. Draft. For more information contact anton@math.binghamton.edu. 2, 17

Rosenbaum, R. and D. B. Rubin (1983). The central role of the propensity score in observational studies for causal effects. Biometrika 70, 41-55. 25

Schick, A. (1993). On efficient estimation in regression models. Ann. Stat. 21, 14861521. 1

Schick, A. (1996a). Root-n consistent and efficient estimation in semiparametric additive regression models. Statistics and Probability Letters 30, 45-51. 28, 160 
Schick, A. (1996b). Root-n consistent estimation in partly linear regression models. Statistics and Probability Letters 28, 353-358. 28

Schick, A. (1996c). Weighted least squares estimates in partly linear regression models. Statistics and Probability Letters 27, 281-287. 50

Wang, Q., O. Linton, and W. Härdle (2004). Semiparametric regression analysis with missing response at random. Journal of the American Statistical Association 99, 334-345. 158

Zhi, D. (2012). Univariate kernel density estimation. Webpage describing method: http://www.stat.duke.edu/ zo2/shared/research/readings/kernelsmoothing.pdf. 17 
APPENDIX 
A. Additional results and tables of MSE

\section{Estimation of $\vartheta$}

Using the model as defined on page 18 where $\vartheta=3, E[\epsilon]=0, X \sim$ Uniform(0,2), with $\epsilon$ and $X$ independent. Then $\hat{\vartheta}$ was calculated using each of the following methods:

1. OLS: Ordinary Least Squares. Estimator will be efficient when the unknown error is in fact normally distributed.

2. OSI: One Step Improvement. Estimator is asymptotically efficient.

3. MELE1: Maximum Empirical Likelihood Estimator with one constraint. For small sample sizes one constraint could be sufficient to acheive efficiency.

4. MELE2: Maximum Empirical Likelihood Estimator with two constraints on the basis. The extra constraint handles larger sample sizes.

5. MELE3: Maximum Empirical Likelihood Estimator with three constraints on the basis. The larger the sample size the more constraints needed to achieve efficiency.

The Mean Square Error (MSE) was calculated for each simulation using various methods of estimating $\vartheta$. Figure 9 shows the MSE for estimating $\vartheta$ when the error are normally distributed. In such a case all the estimators are efficient, and they appear to be very similar. The OSI method needs to estimate the score function, and the Maximum Emperical Likelihood methods use a grid search to find the estimate for $\vartheta$, so the smallest MSE comes from the OLS method. When the errors follow the standard logistic distribution as in Figure 10, more separation can be seen between the methods. OSI happens to do poorly for small sample sizes due to the estimation of the score function, while MELE with one constraint does well. 


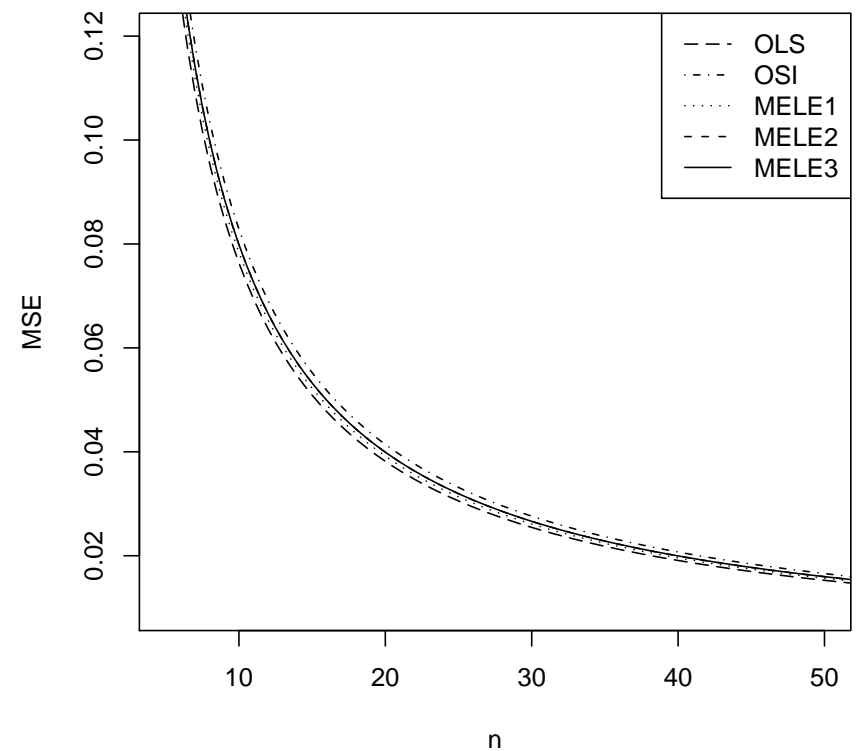

Fig. 9. MSE for various methods of estimating $\vartheta$ under normal errors.

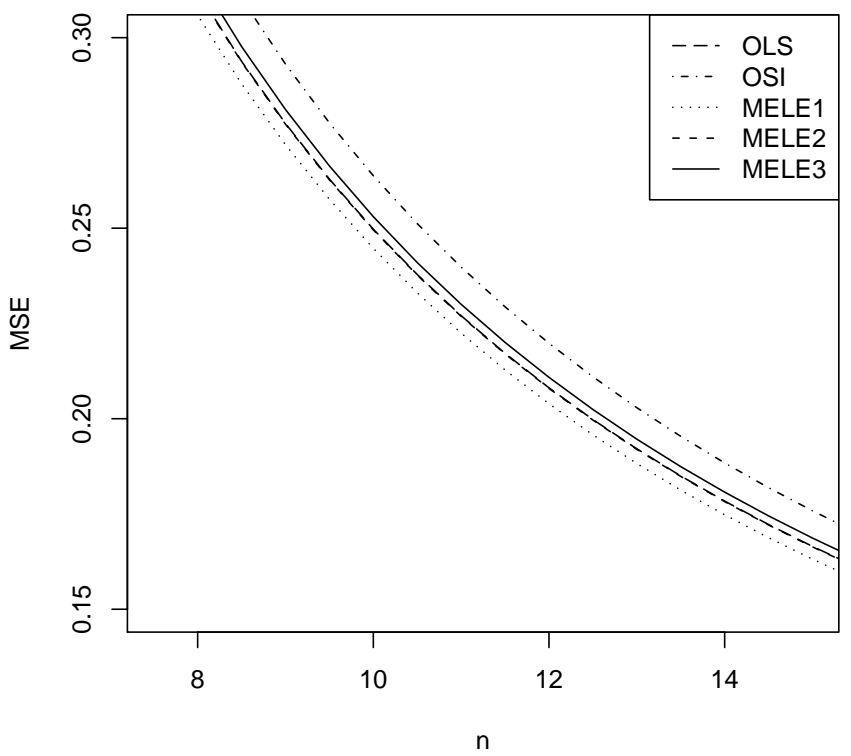

Fig. 10. MSE for various methods of estimating $\vartheta$ under logistic errors. 


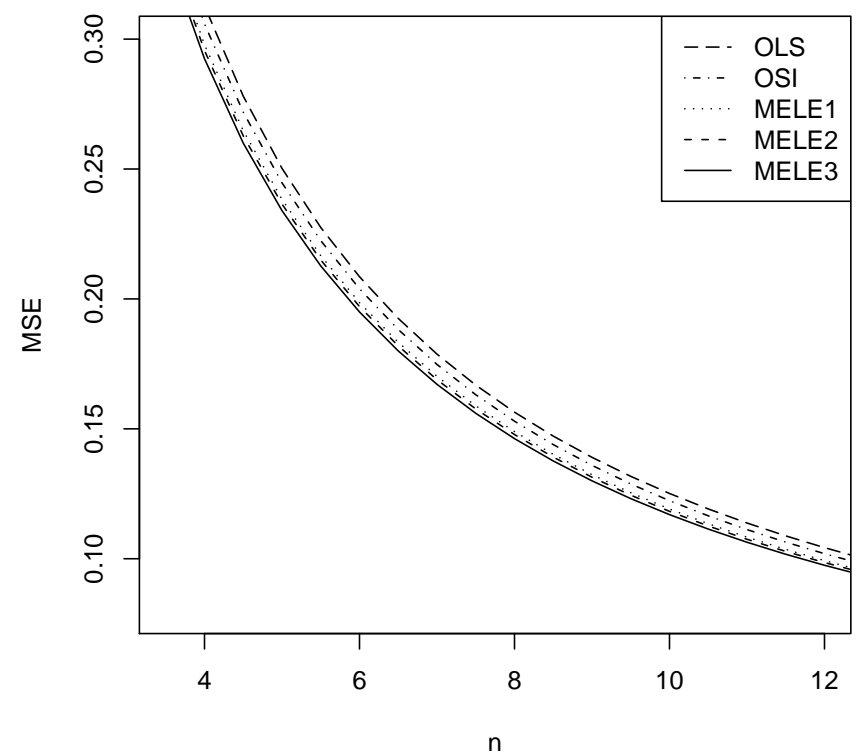

Fig. 11. MSE for various methods of estimating $\vartheta$ under Gumbel errors.

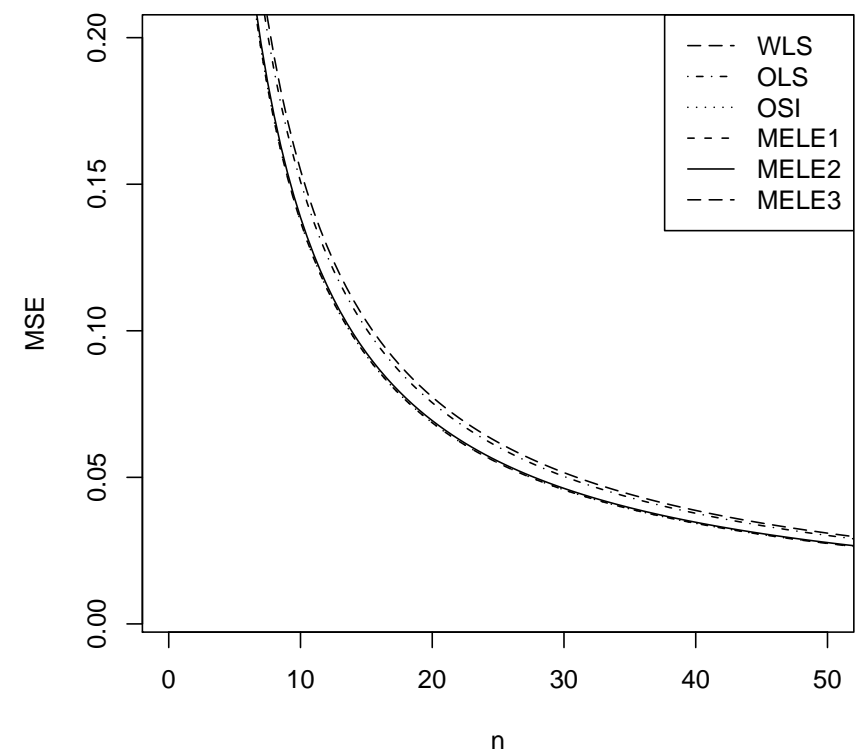

Fig. 12. MSE for various methods of estimating $\vartheta$ under double exponential errors. 
The results from the Gumbel distribution are in Figure 11. The OLS is clearly the least desirable method, while the MELE with three constraints performs the best. For the double exponential model shown in Figure 12 the OLS and OSI methods struggle while the three MELE methods are very close. The results of all these models show that the OLS works well with distributions that are similar to the normal, but departures from normality make the MELE methods preferable. For small sample sizes the OSI method often has more variability than the MELE methods.

Table V shows the values of the MSE averaged over the 20,000 iterations. The simulation used three sample sizes, $N=50, N=500$, and $N=5000$. Then the observed values of MSE were fit to a $1 / N$ model for the plots shown in the paper.

\section{Estimation of $E(Y)$}

Using the model as defined in Chapter $\mathrm{V}$ where the goal is to estimate $E(Y)$ under the simple linear model where $Y=\vartheta X+\epsilon$. Let $X \sim \operatorname{Uniform}(0,2)$, and $\vartheta=3$. The following error distributions of $\epsilon$ are considered:

1. $\epsilon \sim \operatorname{Normal}(0,1)$ where the OLS method is efficient;

2. $\epsilon \sim t$ with 3 degrees of freedom;

3. $\epsilon \sim$ Gamma with a variance of 2 shifted to have a mean of 0 .

4. $\epsilon \sim$ Logistic which under the standard distribution has heavy tails compared to the Normal distribution.

5. $\epsilon \sim$ Gumbel which under the standard distribution is similar to the Normal but is skewed.

For each scenario the asymptotic variances will be compared using the following methods: 
Table V. Simulation results for the estimation of $\vartheta$ from 20,000 iterations.

\begin{tabular}{|c|c|c|c|c|c|c|c|}
\hline & & $\begin{array}{l}\text { Normal } \\
\text { errors }\end{array}$ & $\begin{array}{c}t_{2} \\
\text { errors }\end{array}$ & $\begin{array}{l}\text { Logistic } \\
\text { errors }\end{array}$ & $\begin{array}{c}\text { Gumbel } \\
\text { errors }\end{array}$ & $\begin{array}{c}\text { Gamma } \\
\text { errors }\end{array}$ & $\begin{array}{l}\text { DExp } \\
\text { errors }\end{array}$ \\
\hline \multirow{5}{*}{$\mathrm{N}=50$} & OLS & 0.01527 & 0.1989 & 0.04995 & 0.02503 & 0.03085 & 0.03097 \\
\hline & OSI & 0.01658 & 0.07616 & 0.05278 & 0.02449 & 0.02661 & 0.03022 \\
\hline & MELE1 & 0.01567 & 0.05795 & 0.04895 & 0.02384 & 0.02772 & 0.02747 \\
\hline & MELE2 & 0.01598 & 0.05785 & 0.04992 & 0.02366 & 0.02662 & 0.02746 \\
\hline & MELE3 & 0.01598 & 0.06247 & 0.05062 & 0.02341 & 0.02491 & 0.02776 \\
\hline \multirow{5}{*}{$\mathrm{N}=500$} & OLS & 0.001499 & 0.02289 & 0.004972 & 0.002494 & 0.002986 & 0.002991 \\
\hline & OSI & 0.001542 & 0.007142 & 0.005023 & 0.002245 & 0.002068 & 0.002714 \\
\hline & MELE1 & 0.001521 & 0.006946 & 0.004846 & 0.002329 & 0.002459 & 0.002557 \\
\hline & MELE2 & 0.001527 & 0.006969 & 0.004859 & 0.002278 & 0.002383 & 0.002562 \\
\hline & MELE3 & 0.001523 & 0.006941 & 0.004898 & 0.002258 & 0.002254 & 0.002535 \\
\hline \multirow{5}{*}{$\mathrm{N}=5000$} & OLS & 0.000147 & 0.002705 & 0.0004896 & 0.0002462 & 0.0003038 & 0.0002969 \\
\hline & OSI & 0.0001487 & 0.0008128 & 0.000484 & 0.0002184 & 0.0001849 & 0.0002535 \\
\hline & MELE1 & 0.0001504 & 0.000724 & 0.0004759 & 0.0002281 & 0.0002284 & 0.0002523 \\
\hline & MELE2 & 0.0001505 & 0.0007245 & 0.0004769 & 0.0002228 & 0.0002314 & 0.0002523 \\
\hline & MELE3 & 0.0001486 & 0.0007121 & 0.0004774 & 0.0002203 & 0.0002268 & 0.0002463 \\
\hline
\end{tabular}


1. LD - Listwise Deletion (Equation 3.5);

2. PS - Propensity Score using the true $\pi(X)$ (Equation 3.7);

3. PI_EFF - Partial Imputation with an efficient estimate (Equation 4.3);

4. FI_EFF - Full imputation with an efficient estimate (Equation 4.4);

5. PI_OLS - Partial Imputation using Ordinary Least Squares (Equation 4.15);

6. FI_OLS - Full Imputation using Ordinary Least Squares (Equation 4.16).

There are three different types of missing structures that are considered:

1. None - When there is no missing data;

2. Gaussian - a normal probability of the response being missing as defined in Section B;

3. Exponential - where the probability of the response being missing is higher on one end as defined in Section C,

a. No missing data

The graph for the normally distributed data is shown on page 69. Figure 13 shows the MSE for the estimates of $E(Y)$ when the errors have a t distribution. Figure 14 shows the same for when the errors have a gamma distribution. Figure 15 is for the logistic distribution, and Figure 16 is for the Gumbel distribution.

TableVI shows the MSE values from 20,000 simulations where there is no missing data and the errors are normally distributed. Table VII shows the same thing for errors that have a t distribution, while Table VIII, Table IX] and Table $\mathrm{X}$ have errors that are logistic, Gumbel, and gamma respectively. 


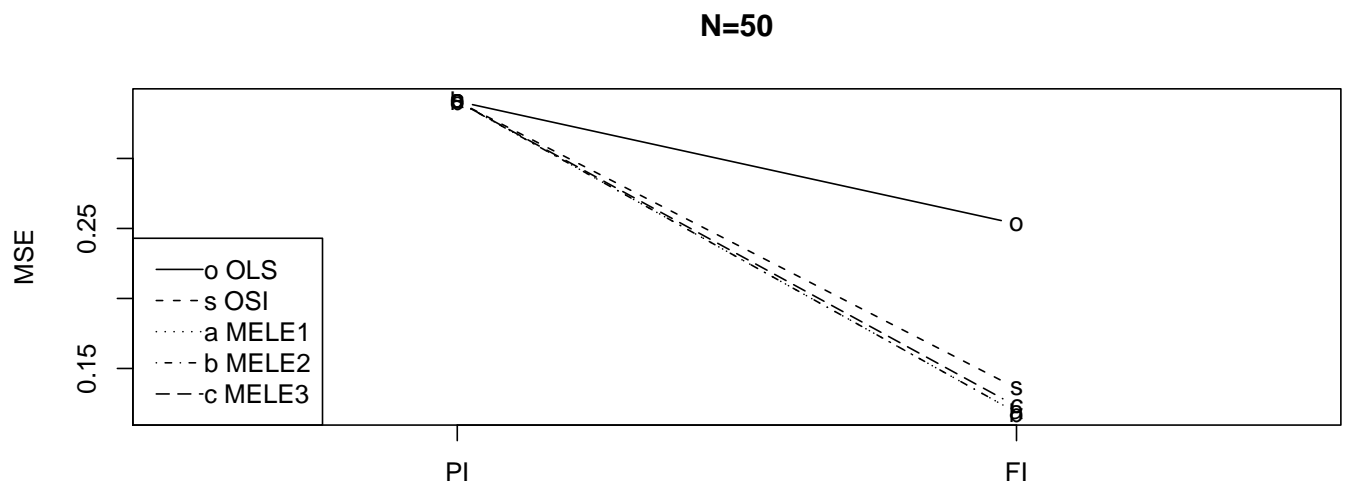

Propensity Score Method $=0.313154$

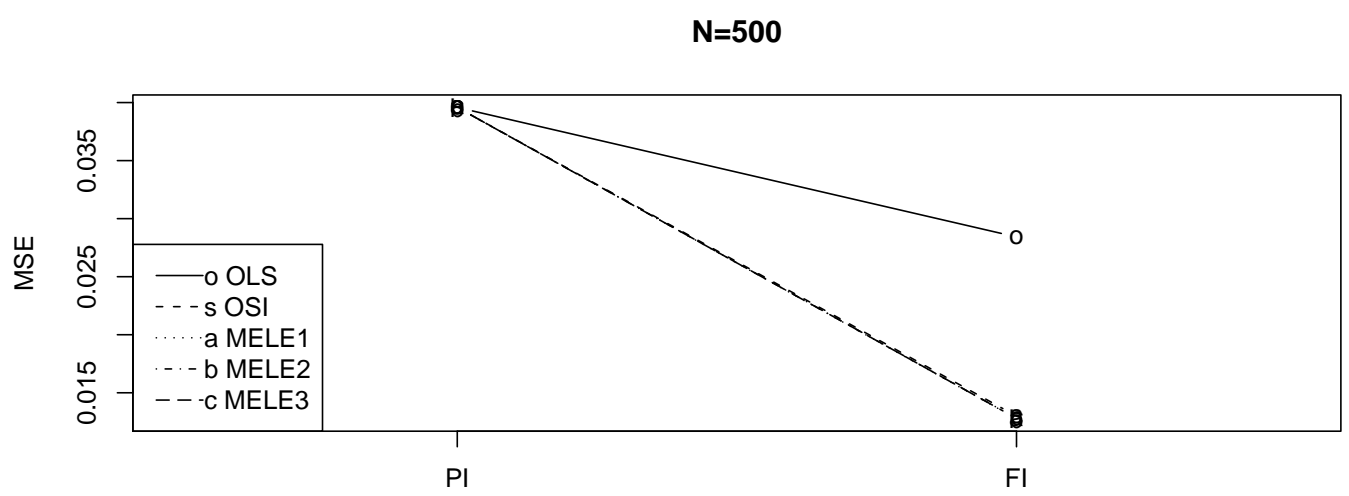

Propensity Score Method $=0.048422$

$\mathrm{N}=5000$

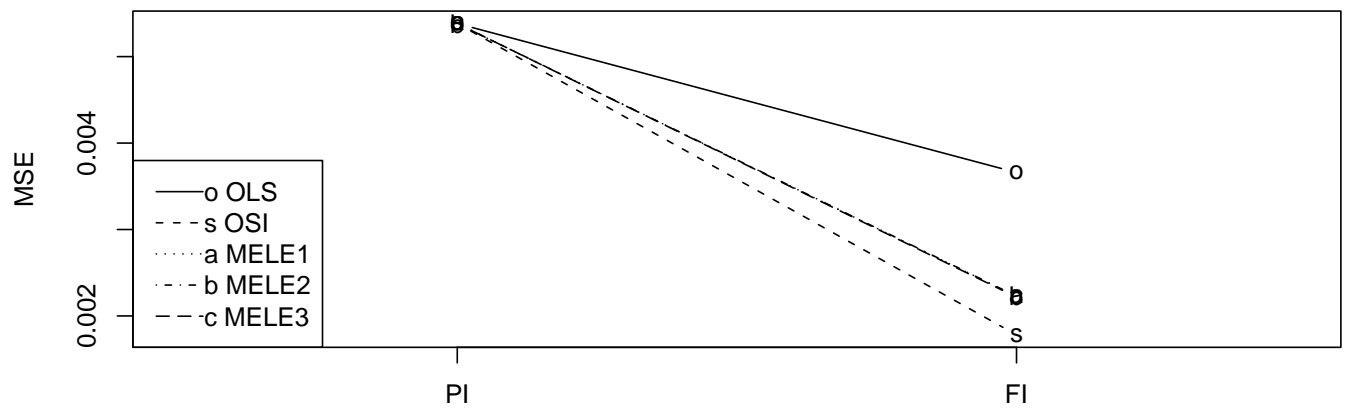

Propensity Score Method $=0.004204$

Fig. 13. MSE for estimating $E[Y]$ where the errors have the t distribution and no missing data 

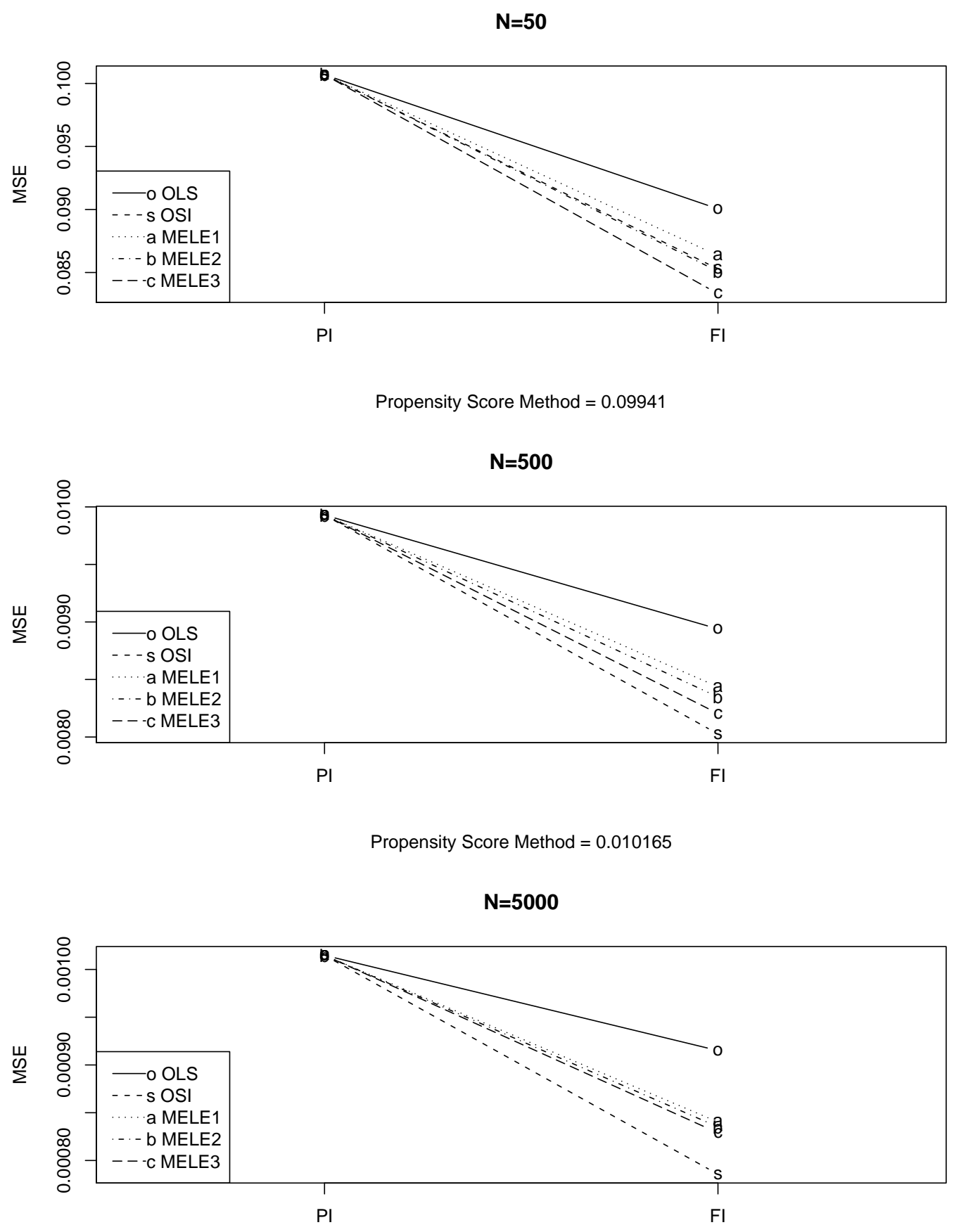

Propensity Score Method $=0.000997$

Fig. 14. MSE for estimating $E[Y]$ where the errors have the gamma distribution and no missing data 

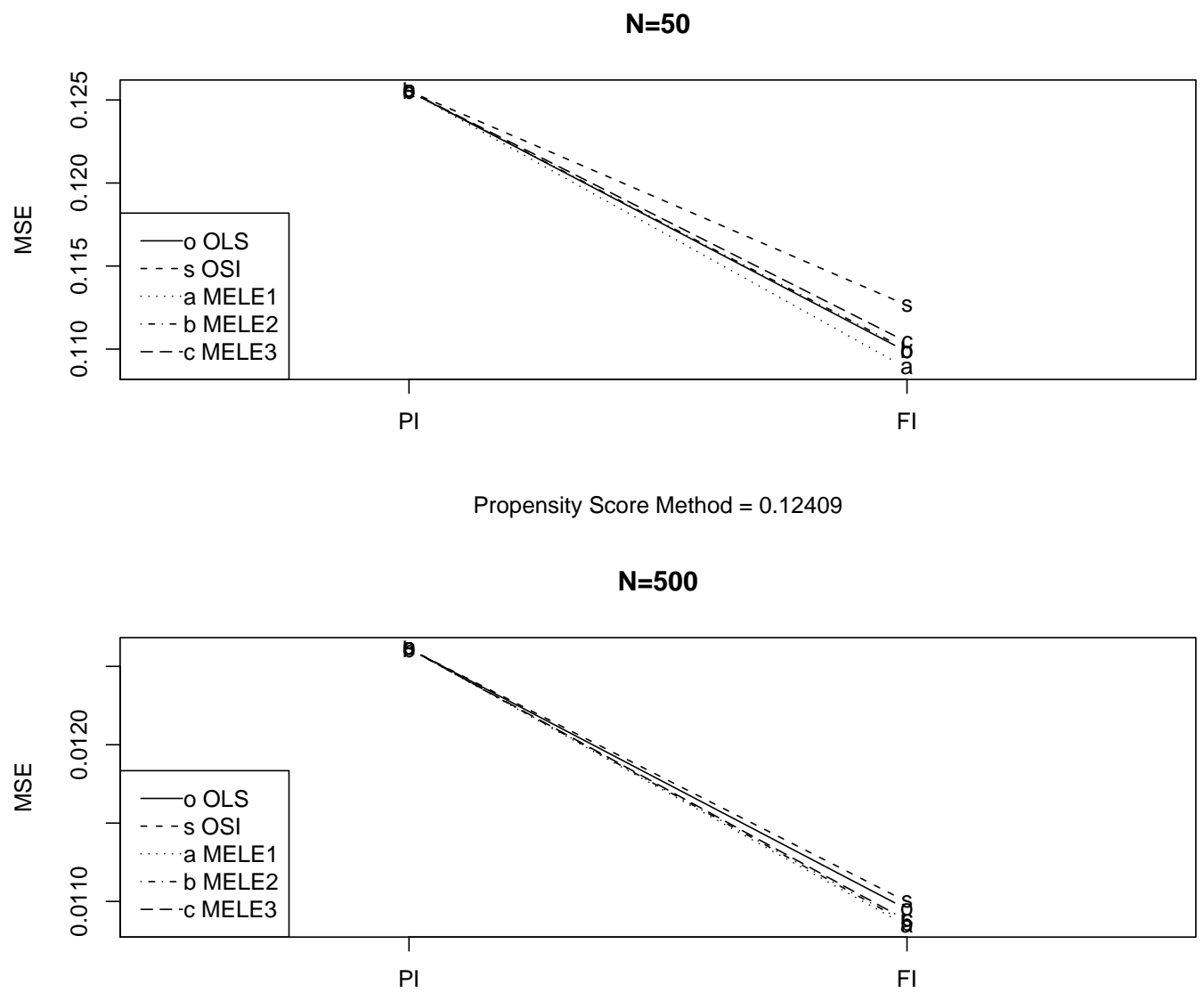

Propensity Score Method $=0.012798$

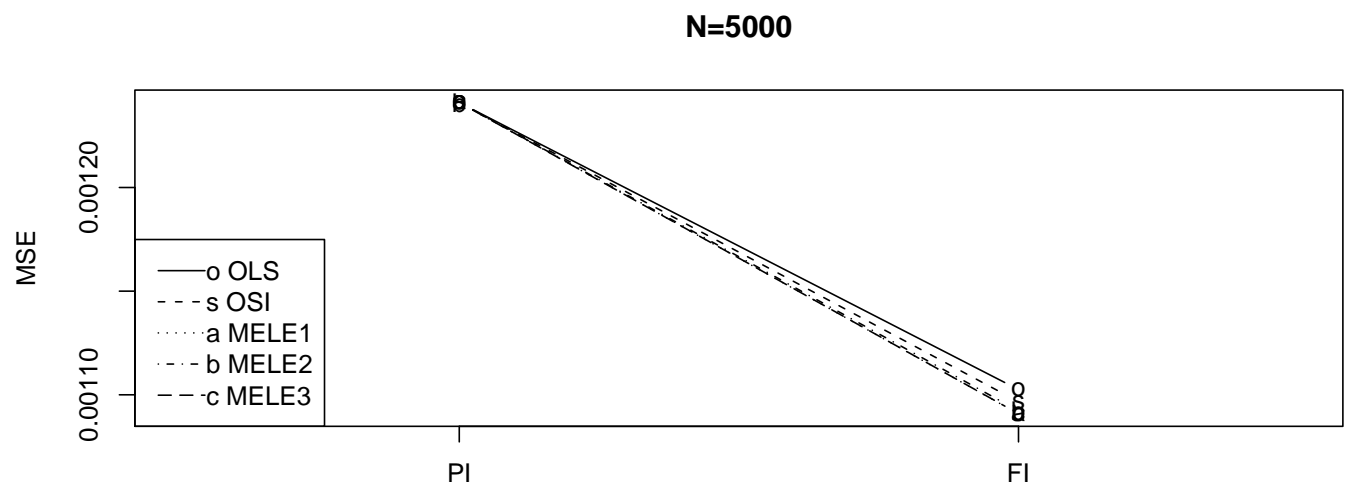

Propensity Score Method $=0.001238$

Fig. 15. MSE for estimating $E[Y]$ where the errors have the logistic distribution and no missing data 

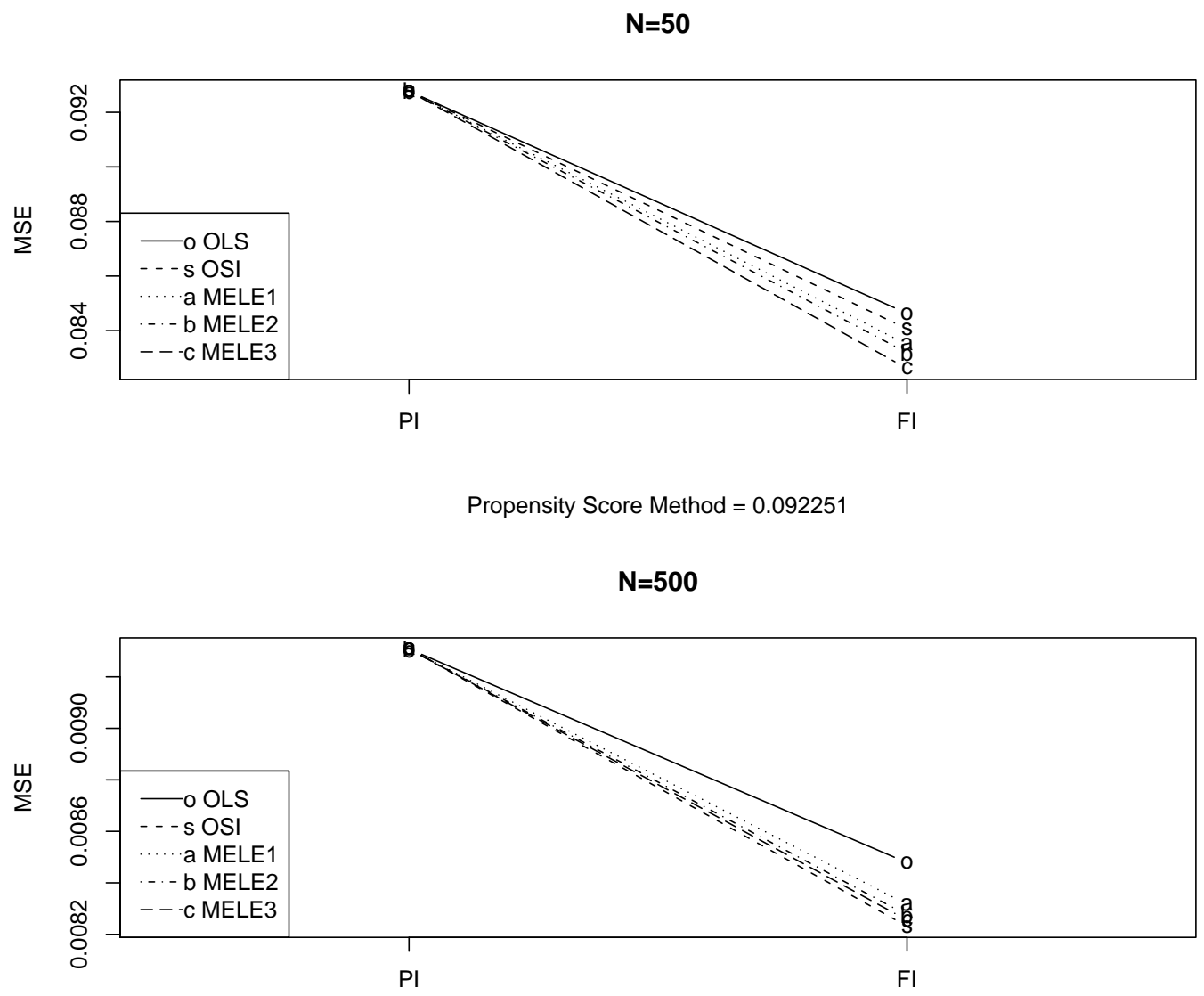

Propensity Score Method $=0.009316$

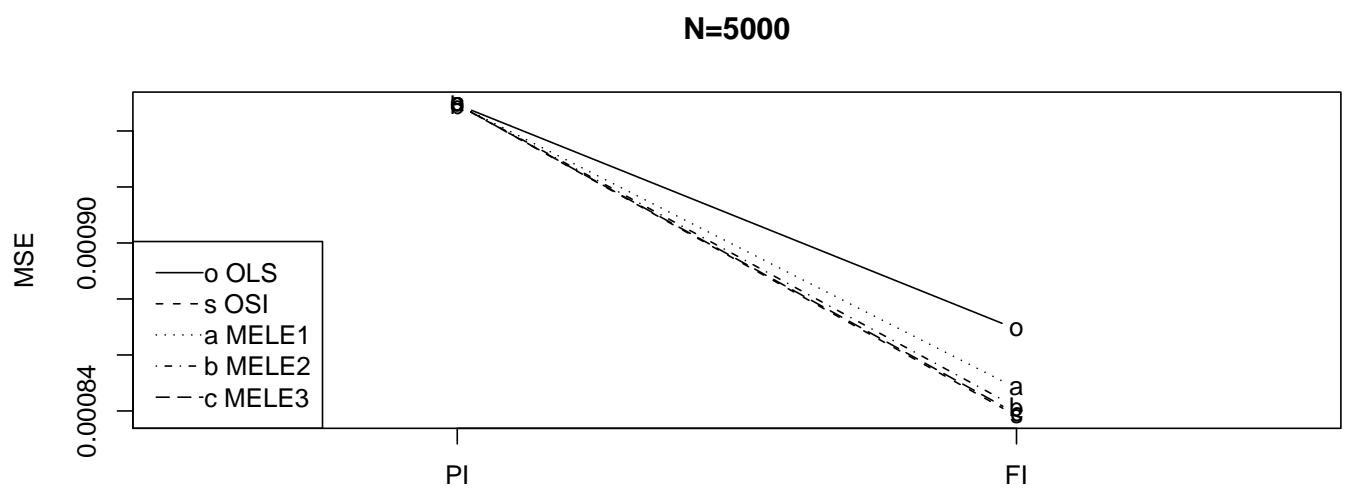

Propensity Score Method $=0.000926$

Fig. 16. MSE for estimating $E[Y]$ where the errors have the Gumbel distribution and no missing data 
Table VI. Simulation results showing the MSE for the estimation of $E[Y]$ where there is no missing data and the errors have a normal distribution

\begin{tabular}{|c|c|c|c|c|}
\hline & & $\begin{array}{c}\text { No } \\
\text { Imputation }\end{array}$ & $\begin{array}{c}\text { Partial } \\
\text { Imputation }\end{array}$ & $\begin{array}{c}\text { Full } \\
\text { Imputation }\end{array}$ \\
\hline \multirow{5}{*}{$\mathrm{N}=50$} & OLS & 0.08017 & 0.08017 & 0.07514 \\
\hline & OSI & 0.08017 & 0.08017 & 0.07642 \\
\hline & MELE1 & 0.08017 & 0.08017 & 0.0754 \\
\hline & MELE2 & 0.08017 & 0.08017 & 0.07566 \\
\hline & MELE3 & 0.08017 & 0.08017 & 0.0756 \\
\hline \multirow{5}{*}{$\mathrm{N}=500$} & OLS & 0.008047 & 0.008047 & 0.007517 \\
\hline & OSI & 0.008047 & 0.008047 & 0.007565 \\
\hline & MELE1 & 0.008047 & 0.008047 & 0.007543 \\
\hline & MELE2 & 0.008047 & 0.008047 & 0.007552 \\
\hline & MELE3 & 0.008047 & 0.008047 & 0.00755 \\
\hline \multirow{5}{*}{$\mathrm{N}=5000$} & OLS & 0.0008031 & 0.0008031 & 0.0007417 \\
\hline & OSI & 0.0008031 & 0.0008031 & 0.0007426 \\
\hline & MELE1 & 0.0008031 & 0.0008031 & 0.0007467 \\
\hline & MELE2 & 0.0008031 & 0.0008031 & 0.0007472 \\
\hline & MELE3 & 0.0008031 & 0.0008031 & 0.0007438 \\
\hline
\end{tabular}


Table VII. Simulation results showing the MSE for the estimation of $E[Y]$ where there is no missing data and the errors have a $t_{2}$ distribution

\begin{tabular}{|c|c|c|c|c|}
\hline & & $\begin{array}{c}\text { No } \\
\text { Imputation }\end{array}$ & $\begin{array}{c}\text { Partial } \\
\text { Imputation }\end{array}$ & $\begin{array}{c}\text { Full } \\
\text { Imputation }\end{array}$ \\
\hline \multirow{5}{*}{$\mathrm{N}=50$} & OLS & 0.3408 & 0.3408 & 0.2533 \\
\hline & OSI & 0.3408 & 0.3408 & 0.1359 \\
\hline & MELE1 & 0.3408 & 0.3408 & 0.1185 \\
\hline & MELE2 & 0.3408 & 0.3408 & 0.1185 \\
\hline & MELE3 & 0.3408 & 0.3408 & 0.123 \\
\hline \multirow{5}{*}{$\mathrm{N}=500$} & OLS & 0.03958 & 0.03958 & 0.02842 \\
\hline & OSI & 0.03958 & 0.03958 & 0.01299 \\
\hline & MELE1 & 0.03958 & 0.03958 & 0.01276 \\
\hline & MELE2 & 0.03958 & 0.03958 & 0.01279 \\
\hline & MELE3 & 0.03958 & 0.03958 & 0.01279 \\
\hline \multirow{5}{*}{$\mathrm{N}=5000$} & OLS & 0.005383 & 0.005383 & 0.003659 \\
\hline & OSI & 0.005383 & 0.005383 & 0.001781 \\
\hline & MELE1 & 0.005383 & 0.005383 & 0.002227 \\
\hline & MELE2 & 0.005383 & 0.005383 & 0.002228 \\
\hline & MELE3 & 0.005383 & 0.005383 & 0.002217 \\
\hline
\end{tabular}


Table VIII. Simulation results showing the MSE for the estimation of $E[Y]$ where there is no missing data and the errors have a logistic distribution

\begin{tabular}{|c|c|c|c|c|}
\hline & & $\begin{array}{l}\text { No } \\
\text { Imputation }\end{array}$ & $\begin{array}{c}\text { Partial } \\
\text { Imputation }\end{array}$ & $\begin{array}{c}\text { Full } \\
\text { Imputation }\end{array}$ \\
\hline \multirow{5}{*}{$\mathrm{N}=50$} & OLS & 0.1255 & 0.1255 & 0.1098 \\
\hline & OSI & 0.1255 & 0.1255 & 0.1126 \\
\hline & MELE1 & 0.1255 & 0.1255 & 0.1088 \\
\hline & MELE2 & 0.1255 & 0.1255 & 0.11 \\
\hline & MELE3 & 0.1255 & 0.1255 & 0.1104 \\
\hline \multirow{5}{*}{$\mathrm{N}=500$} & OLS & 0.01261 & 0.01261 & 0.01095 \\
\hline & OSI & 0.01261 & 0.01261 & 0.011 \\
\hline & MELE1 & 0.01261 & 0.01261 & 0.01084 \\
\hline & MELE2 & 0.01261 & 0.01261 & 0.01086 \\
\hline & MELE3 & 0.01261 & 0.01261 & 0.01088 \\
\hline \multirow{5}{*}{$\mathrm{N}=5000$} & OLS & 0.001241 & 0.001241 & 0.001103 \\
\hline & OSI & 0.001241 & 0.001241 & 0.001097 \\
\hline & MELE1 & 0.001241 & 0.001241 & 0.001091 \\
\hline & MELE2 & 0.001241 & 0.001241 & 0.001092 \\
\hline & MELE3 & 0.001241 & 0.001241 & 0.001091 \\
\hline
\end{tabular}


Table IX. Simulation results showing the MSE for the estimation of $E[Y]$ where there is no missing data and the errors have a Gumbell distribution

\begin{tabular}{|c|c|c|c|c|}
\hline & & $\begin{array}{c}\text { No } \\
\text { Imputation }\end{array}$ & $\begin{array}{c}\text { Partial } \\
\text { Imputation }\end{array}$ & $\begin{array}{c}\text { Full } \\
\text { Imputation }\end{array}$ \\
\hline \multirow{5}{*}{$\mathrm{N}=50$} & OLS & 0.09278 & 0.09278 & 0.08463 \\
\hline & OSI & 0.09278 & 0.09278 & 0.08406 \\
\hline & MELE1 & 0.09278 & 0.09278 & 0.08349 \\
\hline & MELE2 & 0.09278 & 0.09278 & 0.08319 \\
\hline & MELE3 & 0.09278 & 0.09278 & 0.08261 \\
\hline \multirow{5}{*}{$\mathrm{N}=500$} & OLS & 0.009308 & 0.009308 & 0.008478 \\
\hline & OSI & 0.009308 & 0.009308 & 0.008231 \\
\hline & MELE1 & 0.009308 & 0.009308 & 0.008317 \\
\hline & MELE2 & 0.009308 & 0.009308 & 0.008275 \\
\hline & MELE3 & 0.009308 & 0.009308 & 0.008256 \\
\hline \multirow{5}{*}{$\mathrm{N}=5000$} & OLS & 0.0009494 & 0.0009494 & 0.0008692 \\
\hline & OSI & 0.0009494 & 0.0009494 & 0.0008382 \\
\hline & MELE1 & 0.0009494 & 0.0009494 & 0.0008481 \\
\hline & MELE2 & 0.0009494 & 0.0009494 & 0.0008413 \\
\hline & MELE3 & 0.0009494 & 0.0009494 & 0.000839 \\
\hline
\end{tabular}


Table X. Simulation results showing the MSE for the estimation of $E[Y]$ where there is no missing data and the errors have a gamma distribution

\begin{tabular}{|c|c|c|c|c|}
\hline & & $\begin{array}{c}\text { No } \\
\text { Imputation }\end{array}$ & $\begin{array}{c}\text { Partial } \\
\text { Imputation }\end{array}$ & $\begin{array}{c}\text { Full } \\
\text { Imputation }\end{array}$ \\
\hline \multirow{5}{*}{$\mathrm{N}=50$} & OLS & 0.1007 & 0.1007 & 0.09005 \\
\hline & OSI & 0.1007 & 0.1007 & 0.08528 \\
\hline & MELE1 & 0.1007 & 0.1007 & 0.08634 \\
\hline & MELE2 & 0.1007 & 0.1007 & 0.08509 \\
\hline & MELE3 & 0.1007 & 0.1007 & 0.08332 \\
\hline \multirow{5}{*}{$\mathrm{N}=500$} & OLS & 0.009929 & 0.009929 & 0.008939 \\
\hline & OSI & 0.009929 & 0.009929 & 0.008027 \\
\hline & MELE1 & 0.009929 & 0.009929 & 0.008436 \\
\hline & MELE2 & 0.009929 & 0.009929 & 0.008348 \\
\hline & MELE3 & 0.009929 & 0.009929 & 0.008204 \\
\hline \multirow{5}{*}{$\mathrm{N}=5000$} & OLS & 0.001015 & 0.001015 & 0.0009151 \\
\hline & OSI & 0.001015 & 0.001015 & 0.0007856 \\
\hline & MELE1 & 0.001015 & 0.001015 & 0.0008403 \\
\hline & MELE2 & 0.001015 & 0.001015 & 0.0008351 \\
\hline & MELE3 & 0.001015 & 0.001015 & 0.0008289 \\
\hline
\end{tabular}


b. Gaussian missing structure

Figure 17 shows the MSE for the estimates of $E(Y)$ when the errors have a normal distribution. Figure 18 shows the same for when the errors have a t distribution. Figure 19 is for the gamma distribution, Figure 20 is for the logistic distribution, and Figure 21 is for the Gumbel distribution.

Table XI shows the MSE values from 20,000 simulations where the missing structure is Gaussian and the errors are normally distributed. Table XII shows the same thing for errors that have a t distribution, while Table XIII, Table XIV, and Table XV have errors that are logistic, Gumbel, and gamma respectively. 

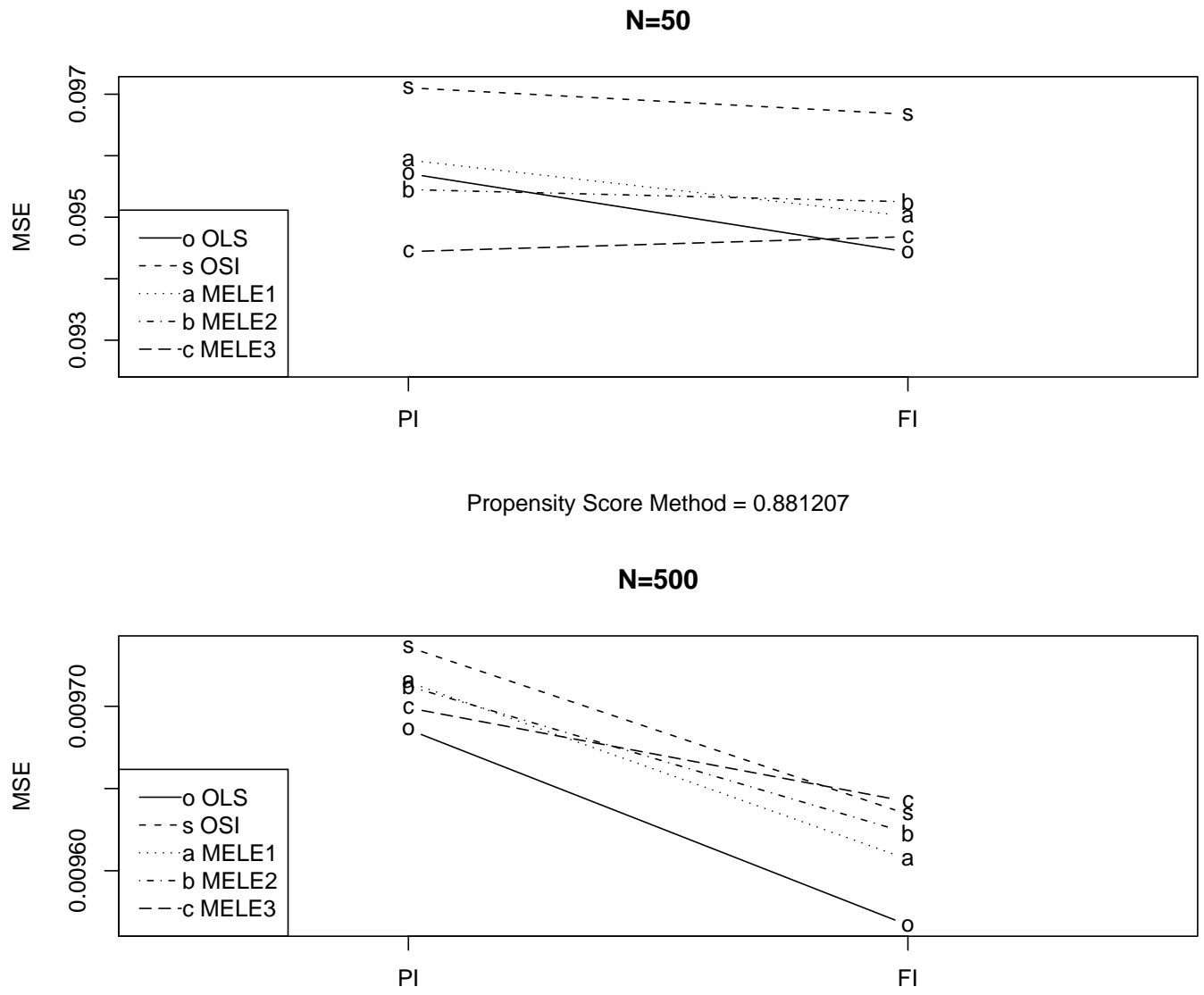

Propensity Score Method $=0.094293$

$\mathrm{N}=5000$

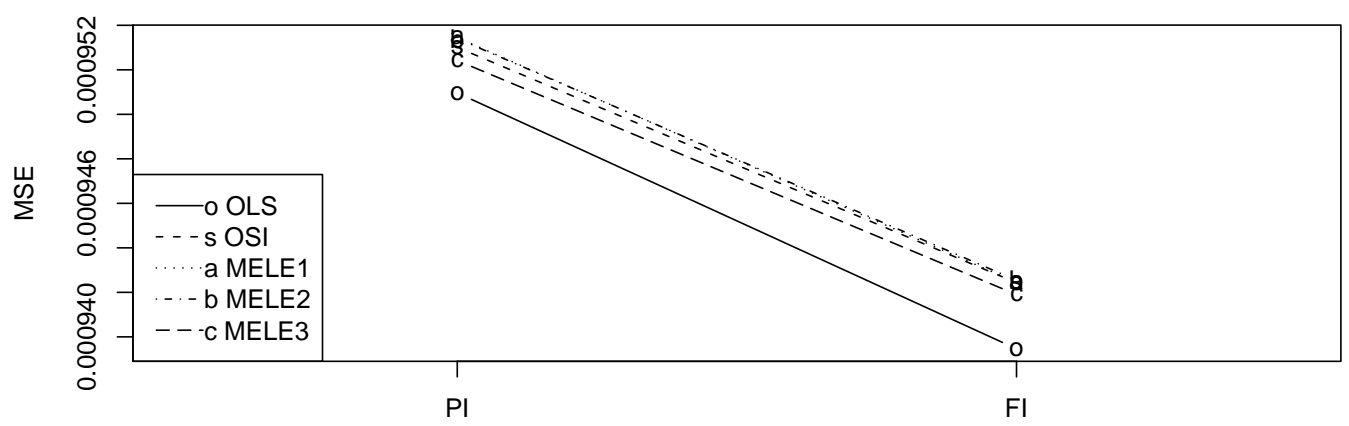

Propensity Score Method $=0.009639$

Fig. 17. MSE for estimating $E[Y]$ where the errors have the normal distribution and a Gaussian missingness structure 

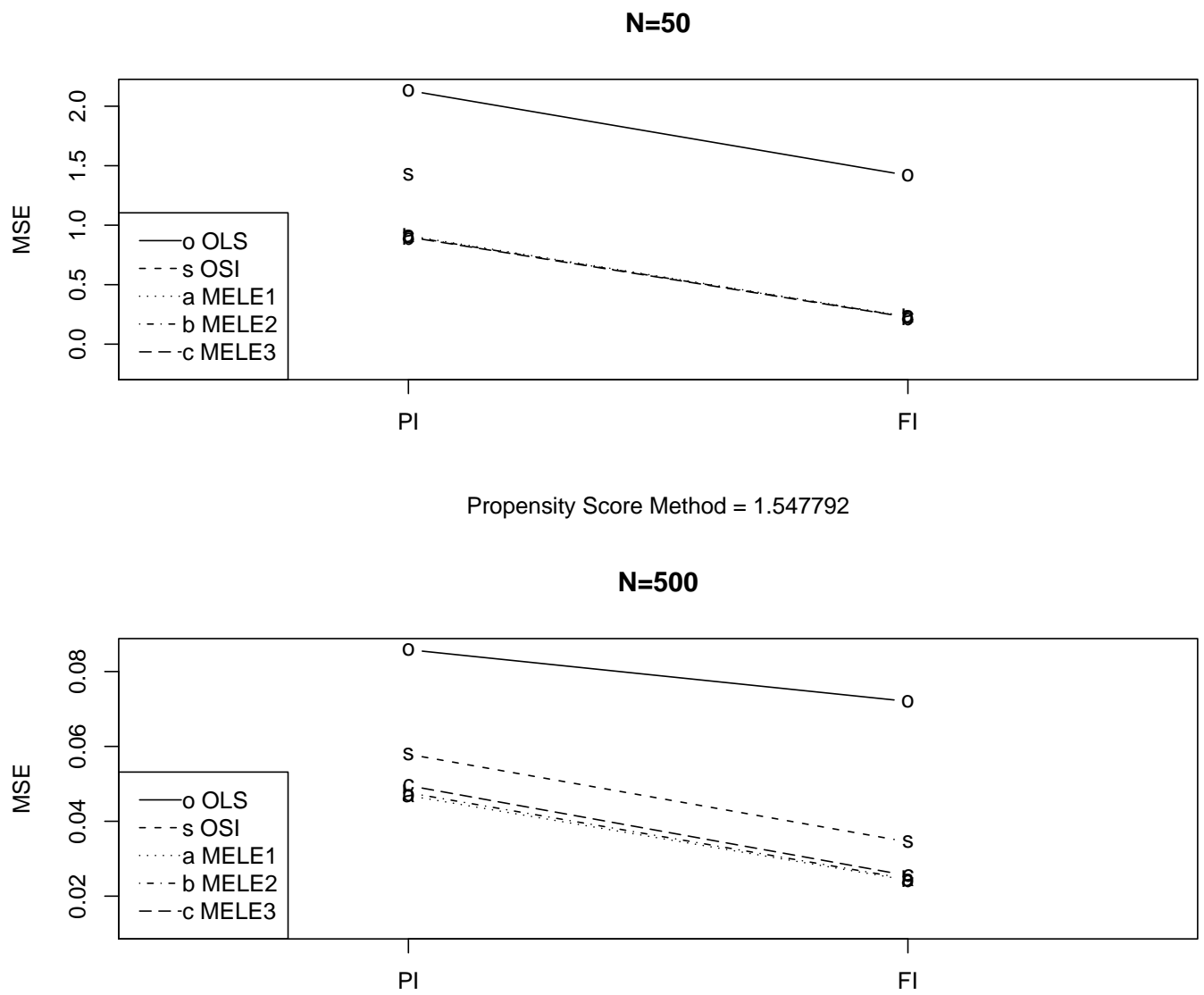

Propensity Score Method $=0.312226$

$\mathrm{N}=5000$

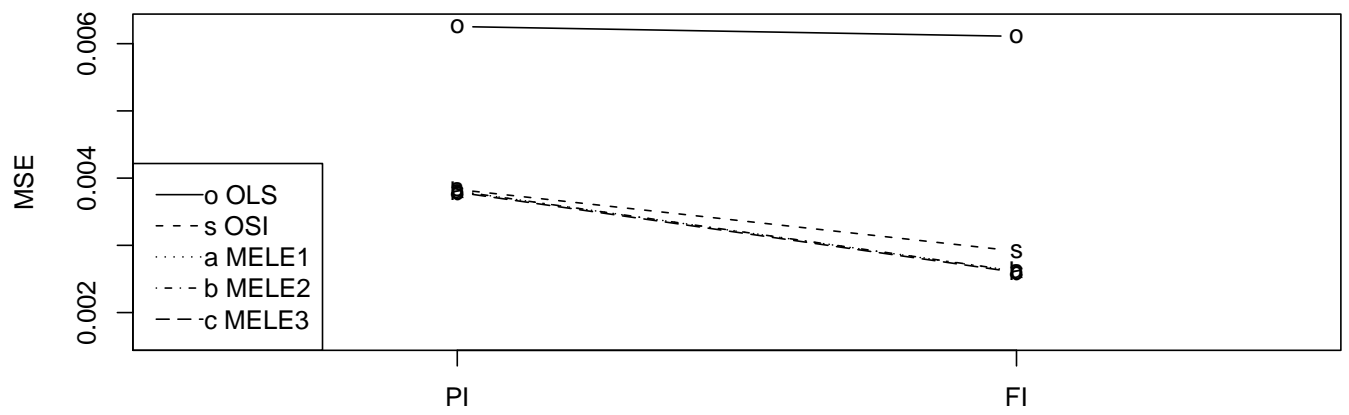

Propensity Score Method $=0.021554$

Fig. 18. MSE for estimating $E[Y]$ where the errors have the t distribution and a Gaussian missingness structure 

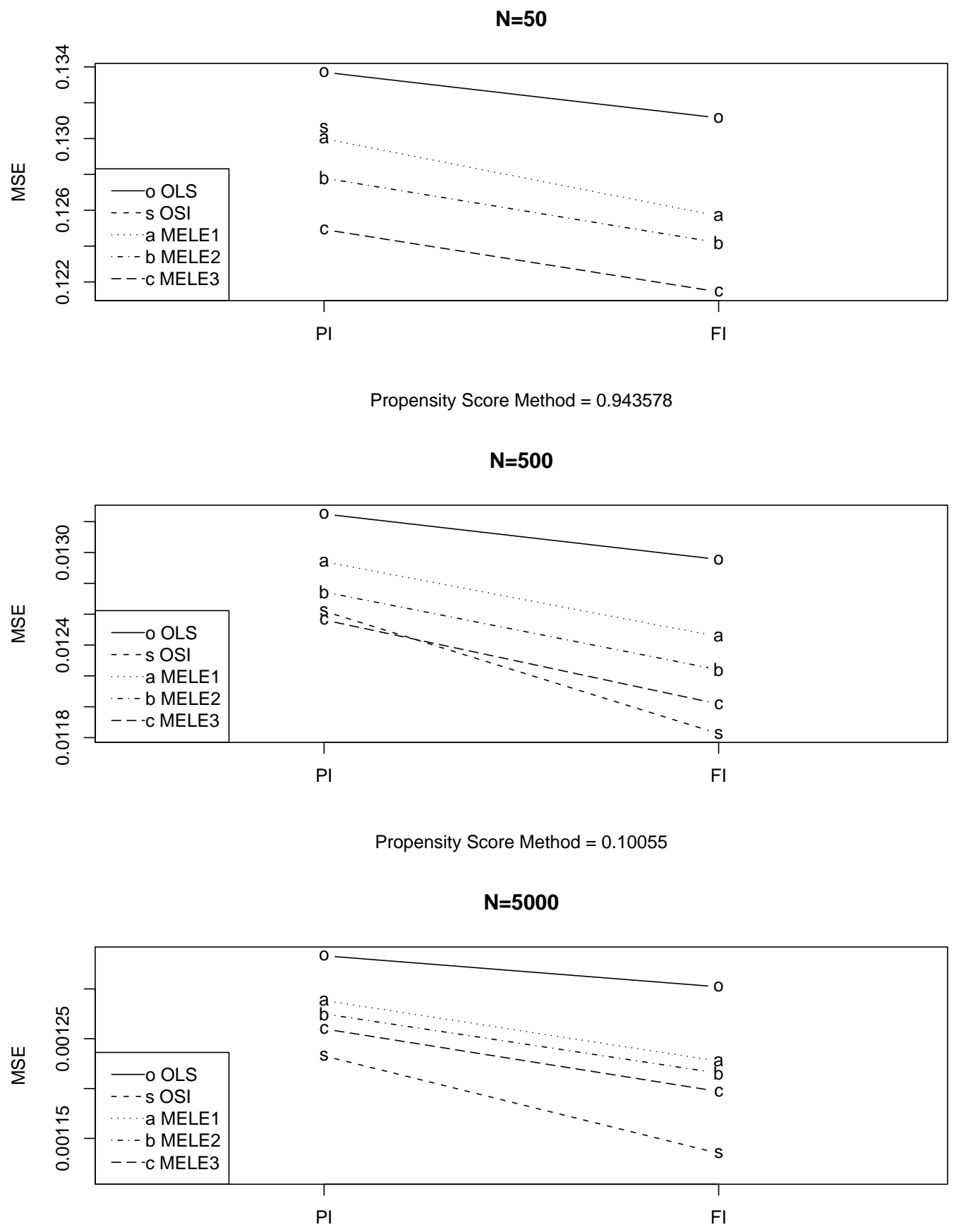

Propensity Score Method $=0.010128$

Fig. 19. MSE for estimating $E[Y]$ where the errors have the gamma distribution and a Gaussian missingness structure 

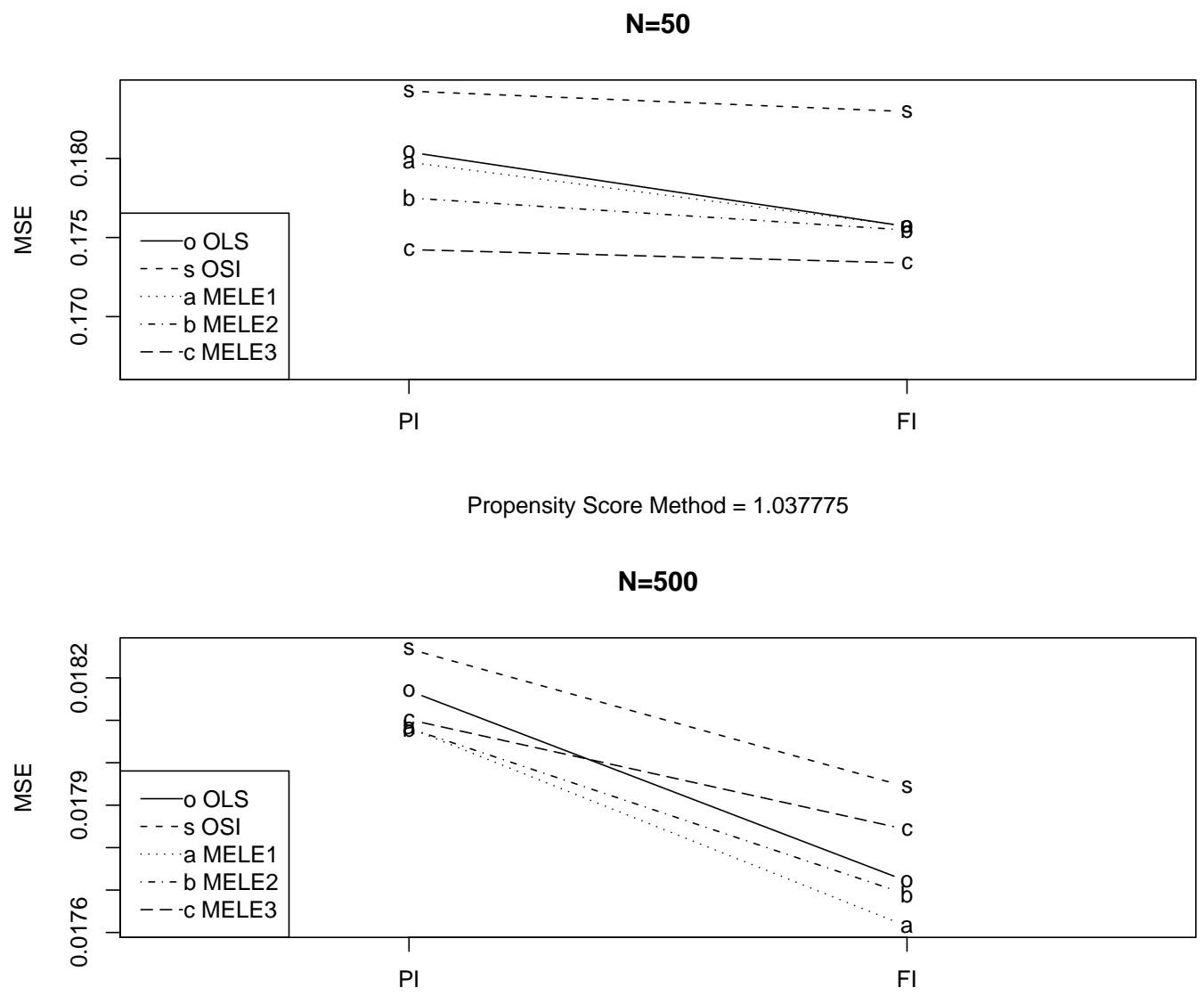

Propensity Score Method $=0.111432$

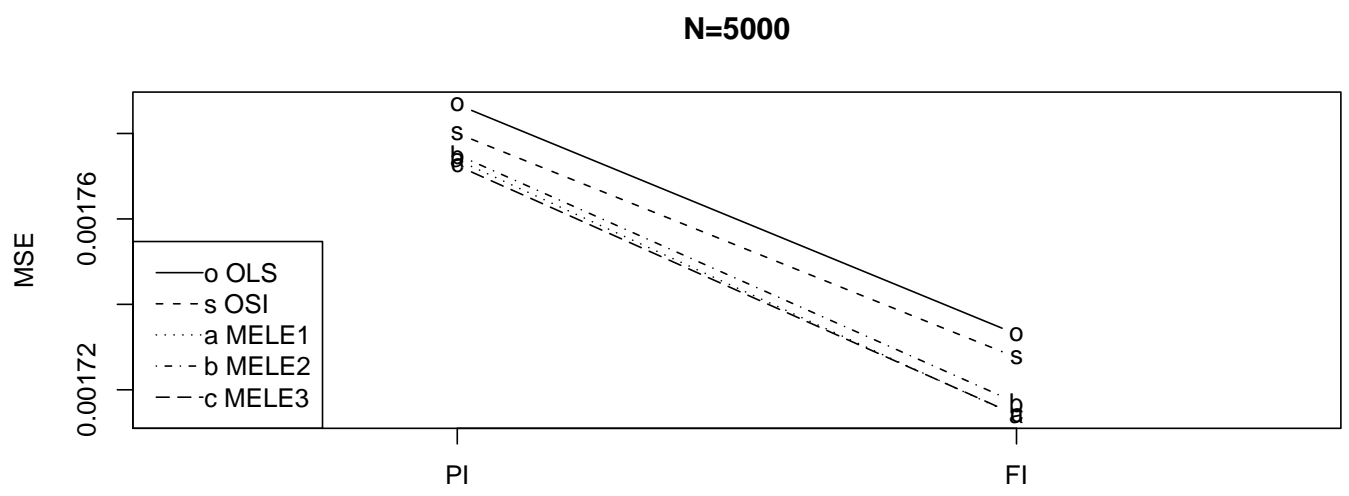

Propensity Score Method $=0.011279$

Fig. 20. MSE for estimating $E[Y]$ where the errors have the logistic distribution and a Gaussian missingness structure 

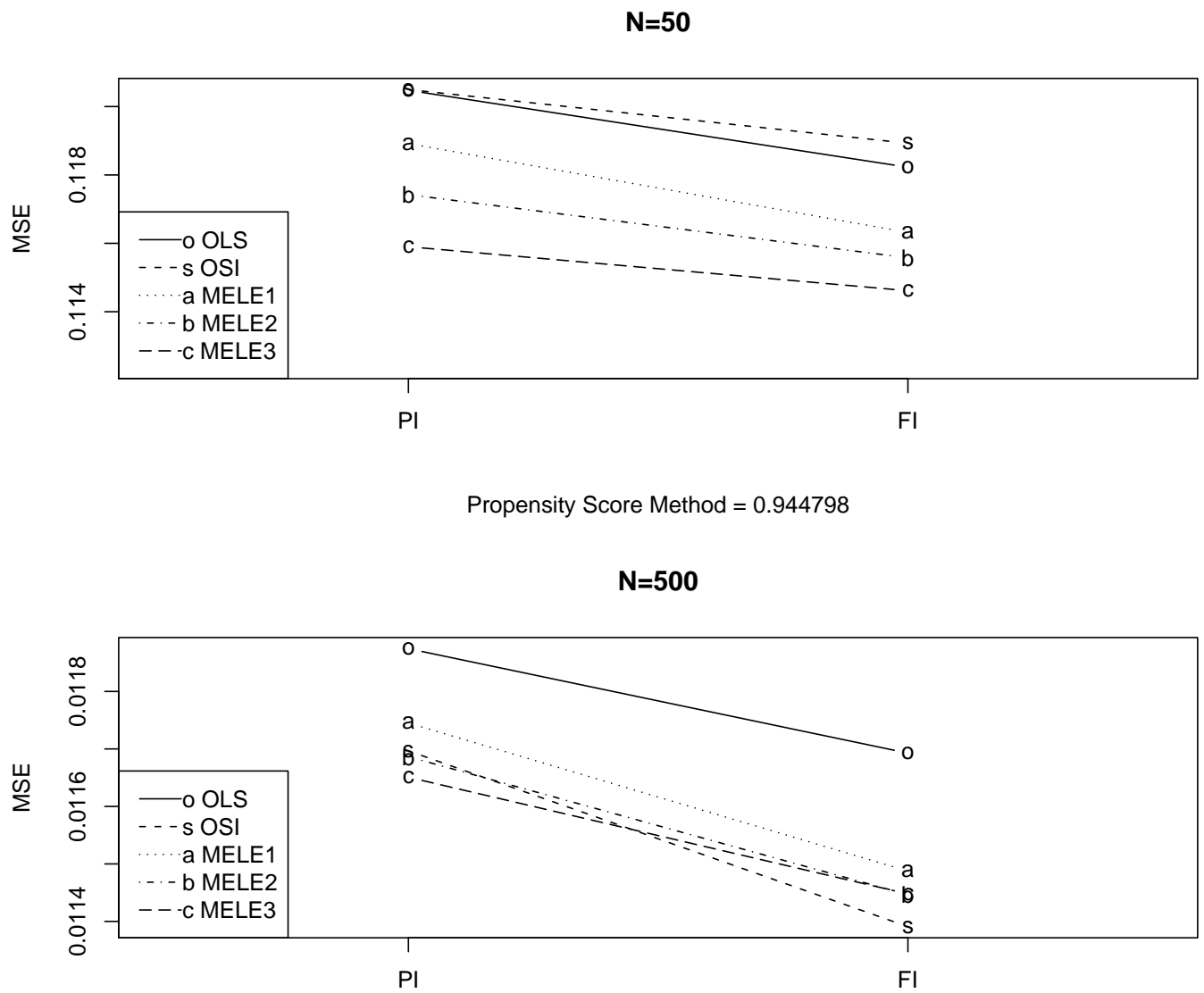

Propensity Score Method $=0.099831$

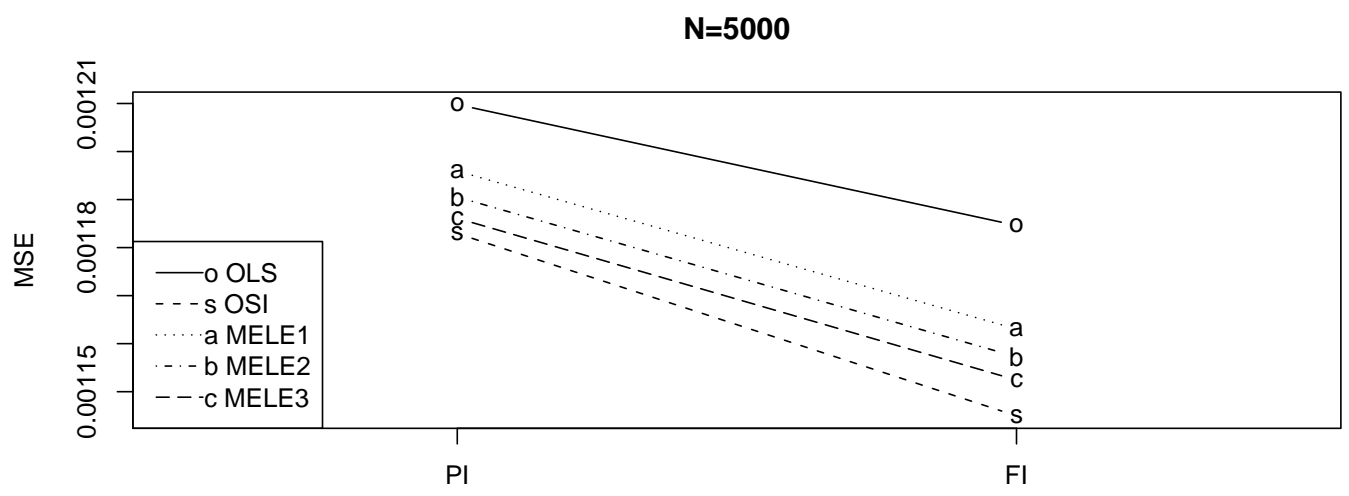

Propensity Score Method $=0.009751$

Fig. 21. MSE for estimating $E[Y]$ where the errors have the Gumbel distribution and a Gaussian missingness structure 
Table XI. Simulation results showing the MSE for the estimation of $E[Y]$ where the missing structure is Gaussian and the errors have a normal distribution

\begin{tabular}{|c|c|c|c|c|}
\hline & & $\begin{array}{c}\text { No } \\
\text { Imputation }\end{array}$ & $\begin{array}{c}\text { Partial } \\
\text { Imputation }\end{array}$ & $\begin{array}{c}\text { Full } \\
\text { Imputation }\end{array}$ \\
\hline \multirow{5}{*}{$\mathrm{N}=50$} & OLS & 0.1777 & 0.09571 & 0.09443 \\
\hline & OSI & 0.1777 & 0.0971 & 0.09667 \\
\hline & MELE1 & 0.1777 & 0.09593 & 0.09502 \\
\hline & MELE2 & 0.1777 & 0.09545 & 0.09525 \\
\hline & MELE3 & 0.1777 & 0.09444 & 0.09468 \\
\hline \multirow{5}{*}{$\mathrm{N}=500$} & OLS & 0.01806 & 0.009686 & 0.009567 \\
\hline & OSI & 0.01806 & 0.009736 & 0.009634 \\
\hline & MELE1 & 0.01806 & 0.009715 & 0.009607 \\
\hline & MELE2 & 0.01806 & 0.009712 & 0.009623 \\
\hline & MELE3 & 0.01806 & 0.009699 & 0.009642 \\
\hline \multirow{5}{*}{$\mathrm{N}=5000$} & OLS & 0.001776 & 0.000951 & 0.0009395 \\
\hline & OSI & 0.001776 & 0.000953 & 0.0009424 \\
\hline & MELE1 & 0.001776 & 0.0009535 & 0.0009424 \\
\hline & MELE2 & 0.001776 & 0.0009534 & 0.0009425 \\
\hline & MELE3 & 0.001776 & 0.0009524 & 0.0009419 \\
\hline
\end{tabular}


Table XII. Simulation results showing the MSE for the estimation of $E[Y]$ where the missing structure is Gaussian and the errors have a $t_{2}$ distribution

\begin{tabular}{|c|c|c|c|c|}
\hline & & $\begin{array}{c}\text { No } \\
\text { Imputation }\end{array}$ & $\begin{array}{c}\text { Partial } \\
\text { Imputation }\end{array}$ & $\begin{array}{c}\text { Full } \\
\text { Imputation }\end{array}$ \\
\hline \multirow{5}{*}{$\mathrm{N}=50$} & OLS & 3.387 & 2.132 & 1.418 \\
\hline & OSI & 3.387 & 1.431 & $\mathrm{NaN}$ \\
\hline & MELE1 & 3.387 & 0.9123 & 0.2325 \\
\hline & MELE2 & 3.387 & 0.9055 & 0.2284 \\
\hline & MELE3 & 3.387 & 0.9005 & 0.2254 \\
\hline \multirow{5}{*}{$\mathrm{N}=500$} & OLS & 0.115 & 0.08583 & 0.07203 \\
\hline & OSI & 0.115 & 0.05789 & 0.03451 \\
\hline & MELE1 & 0.115 & 0.04686 & 0.02426 \\
\hline & MELE2 & 0.115 & 0.04762 & 0.02459 \\
\hline & MELE3 & 0.115 & 0.04953 & 0.02549 \\
\hline \multirow{5}{*}{$\mathrm{N}=5000$} & OLS & 0.007591 & 0.006255 & 0.006109 \\
\hline & OSI & 0.007591 & 0.003831 & 0.002914 \\
\hline & MELE1 & 0.007591 & 0.003808 & 0.002613 \\
\hline & MELE2 & 0.007591 & 0.003808 & 0.002616 \\
\hline & MELE3 & 0.007591 & 0.003791 & 0.002598 \\
\hline
\end{tabular}


Table XIII. Simulation results showing the MSE for the estimation of $E[Y]$ where the missing structure is Gaussian and the errors have a logistic distribution

\begin{tabular}{|c|c|c|c|c|}
\hline & & $\begin{array}{c}\text { No } \\
\text { Imputation }\end{array}$ & $\begin{array}{c}\text { Partial } \\
\text { Imputation }\end{array}$ & $\begin{array}{c}\text { Full } \\
\text { Imputation }\end{array}$ \\
\hline \multirow{5}{*}{$\mathrm{N}=50$} & OLS & 0.2719 & 0.1804 & 0.1757 \\
\hline & OSI & 0.2719 & 0.1843 & 0.183 \\
\hline & MELE1 & 0.2719 & 0.1798 & 0.1757 \\
\hline & MELE2 & 0.2719 & 0.1775 & 0.1755 \\
\hline & MELE3 & 0.2719 & 0.1742 & 0.1734 \\
\hline \multirow{5}{*}{$\mathrm{N}=500$} & OLS & 0.02746 & 0.01817 & 0.01772 \\
\hline & OSI & 0.02746 & 0.01827 & 0.01794 \\
\hline & MELE1 & 0.02746 & 0.01808 & 0.01761 \\
\hline & MELE2 & 0.02746 & 0.01808 & 0.01769 \\
\hline & MELE3 & 0.02746 & 0.0181 & 0.01784 \\
\hline \multirow{5}{*}{$\mathrm{N}=5000$} & OLS & 0.002713 & 0.001787 & 0.001733 \\
\hline & OSI & 0.002713 & 0.00178 & 0.001728 \\
\hline & MELE1 & 0.002713 & 0.001774 & 0.001714 \\
\hline & MELE2 & 0.002713 & 0.001775 & 0.001717 \\
\hline & MELE3 & 0.002713 & 0.001772 & 0.001714 \\
\hline
\end{tabular}


Table XIV. Simulation results showing the MSE for the estimation of $E[Y]$ where the missing structure is Gaussian and the errors have a Gumbel distribution

\begin{tabular}{|c|c|c|c|c|}
\hline & & $\begin{array}{c}\text { No } \\
\text { Imputation }\end{array}$ & $\begin{array}{c}\text { Partial } \\
\text { Imputation }\end{array}$ & $\begin{array}{c}\text { Full } \\
\text { Imputation }\end{array}$ \\
\hline \multirow{5}{*}{$\mathrm{N}=50$} & OLS & 0.2059 & 0.1205 & 0.1182 \\
\hline & OSI & 0.2059 & 0.1205 & 0.1189 \\
\hline & MELE1 & 0.2059 & 0.1189 & 0.1163 \\
\hline & MELE2 & 0.2059 & 0.1174 & 0.1156 \\
\hline & MELE3 & 0.2059 & 0.1159 & 0.1146 \\
\hline \multirow{5}{*}{$\mathrm{N}=500$} & OLS & 0.02063 & 0.01187 & 0.01169 \\
\hline & OSI & 0.02063 & 0.0117 & 0.01139 \\
\hline & MELE1 & 0.02063 & 0.01175 & 0.01149 \\
\hline & MELE2 & 0.02063 & 0.01169 & 0.01145 \\
\hline & MELE3 & 0.02063 & 0.01165 & 0.01145 \\
\hline \multirow{5}{*}{$\mathrm{N}=5000$} & OLS & 0.002079 & 0.00121 & 0.001185 \\
\hline & OSI & 0.002079 & 0.001183 & 0.001145 \\
\hline & MELE1 & 0.002079 & 0.001196 & 0.001163 \\
\hline & MELE2 & 0.002079 & 0.001191 & 0.001157 \\
\hline & MELE3 & 0.002079 & 0.001186 & 0.001152 \\
\hline
\end{tabular}


Table XV. Simulation results showing the MSE for the estimation of $E[Y]$ where the missing structure is Gaussian and the errors have a gamma distribution

\begin{tabular}{|c|c|c|c|c|}
\hline & & $\begin{array}{c}\text { No } \\
\text { Imputation }\end{array}$ & $\begin{array}{c}\text { Partial } \\
\text { Imputation }\end{array}$ & $\begin{array}{c}\text { Full } \\
\text { Imputation }\end{array}$ \\
\hline \multirow{5}{*}{$\mathrm{N}=50$} & OLS & 0.2193 & 0.1337 & 0.1312 \\
\hline & OSI & 0.2193 & 0.1306 & 0.06195 \\
\hline & MELE1 & 0.2193 & 0.13 & 0.1257 \\
\hline & MELE2 & 0.2193 & 0.1278 & 0.1242 \\
\hline & MELE3 & 0.2193 & 0.1249 & 0.1214 \\
\hline \multirow{5}{*}{$\mathrm{N}=500$} & OLS & 0.02198 & 0.01325 & 0.01295 \\
\hline & OSI & 0.02198 & 0.01262 & 0.01183 \\
\hline & MELE1 & 0.02198 & 0.01294 & 0.01245 \\
\hline & MELE2 & 0.02198 & 0.01274 & 0.01224 \\
\hline & MELE3 & 0.02198 & 0.01256 & 0.01202 \\
\hline \multirow{5}{*}{$\mathrm{N}=5000$} & OLS & 0.002214 & 0.001333 & 0.001302 \\
\hline & OSI & 0.002214 & 0.001233 & 0.001135 \\
\hline & MELE1 & 0.002214 & 0.001288 & 0.001228 \\
\hline & MELE2 & 0.002214 & 0.001275 & 0.001215 \\
\hline & MELE3 & 0.002214 & 0.00126 & 0.001197 \\
\hline
\end{tabular}


c. Exponential missing structure

The graph for the normally distributed data is shown on page 70, and the graph for the errors with the gamma distribution is on page 72 . Figure 13 shows the MSE for the estimates of $E(Y)$ when the errors have a t distribution. Figure 15 is for the logistic distribution, and Figure 16 is for the Gumbel distribution.

Table XVI shows the MSE values from 20,000 simulations where the missing structure is exponential and the errors are normally distributed. Table XVII shows the same thing for errors that have a t distribution, while Table XVIII, Table XIX, and Table XX have errors that are logistic, Gumbel, and gamma respectively. 

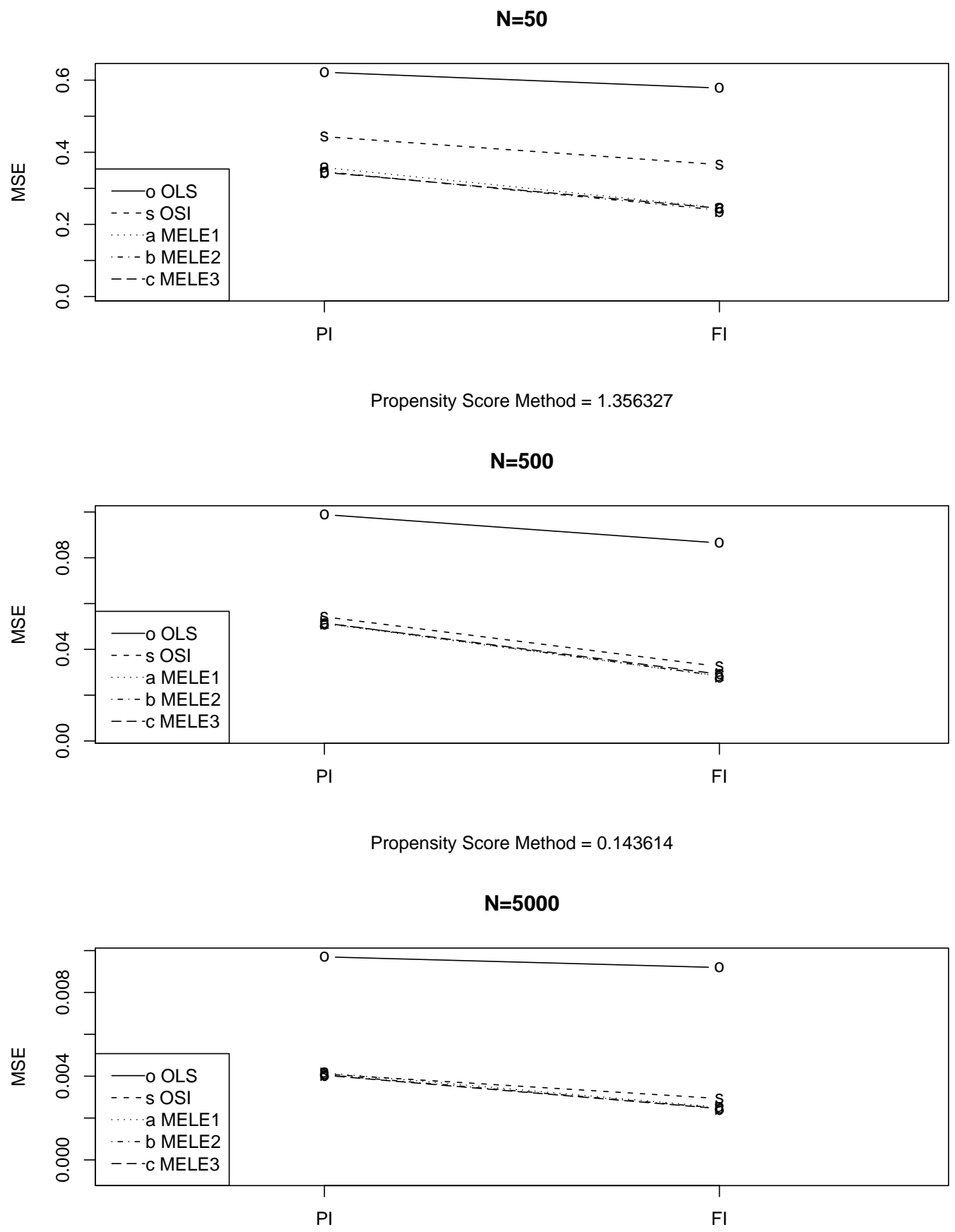

Propensity Score Method $=0.028612$

Fig. 22. MSE for estimating $E[Y]$ where the errors have the t distribution and an exponential missing structure 

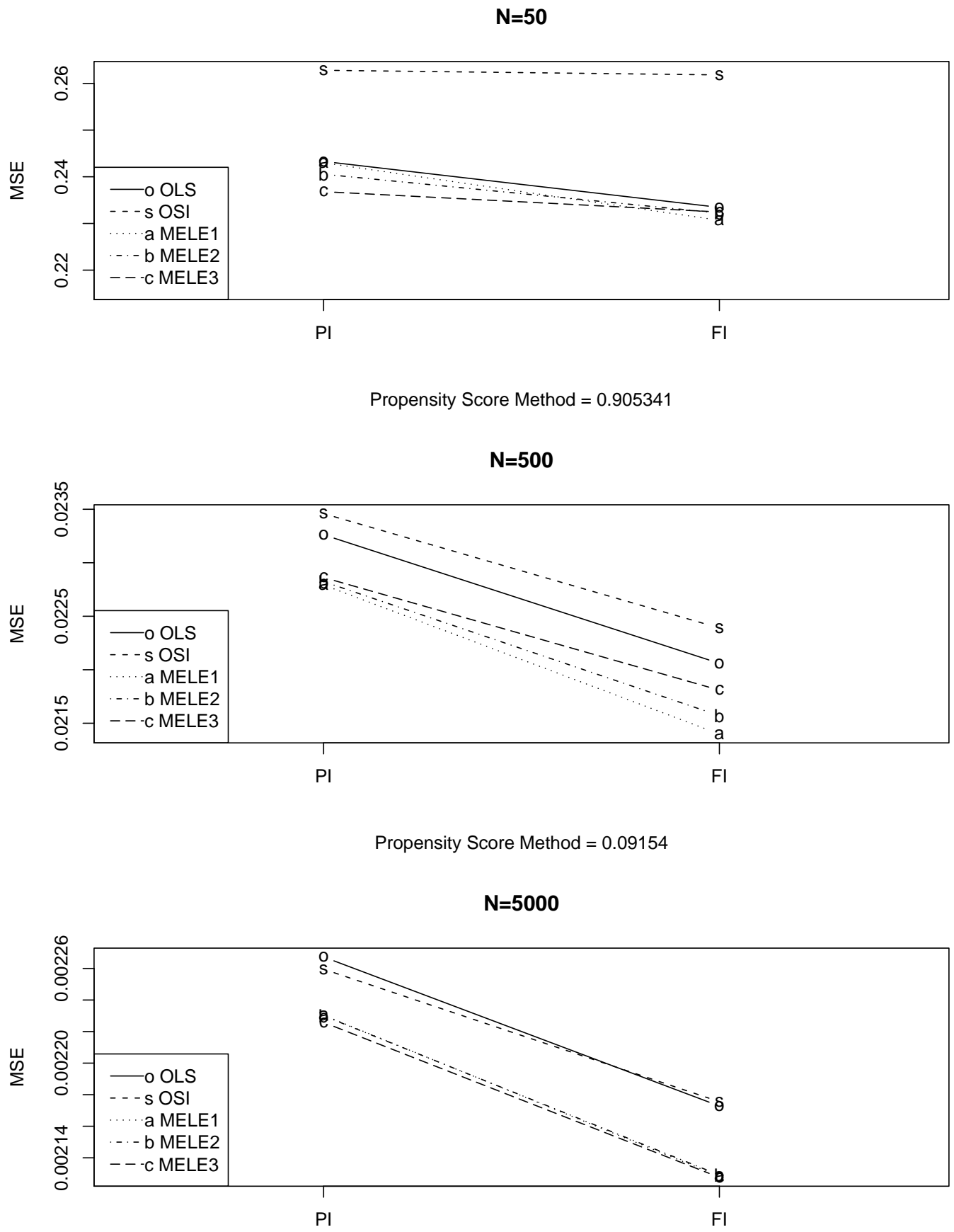

Propensity Score Method $=0.009117$

Fig. 23. MSE for estimating $E[Y]$ where the errors have the logistic distribution and an exponential missing structure 

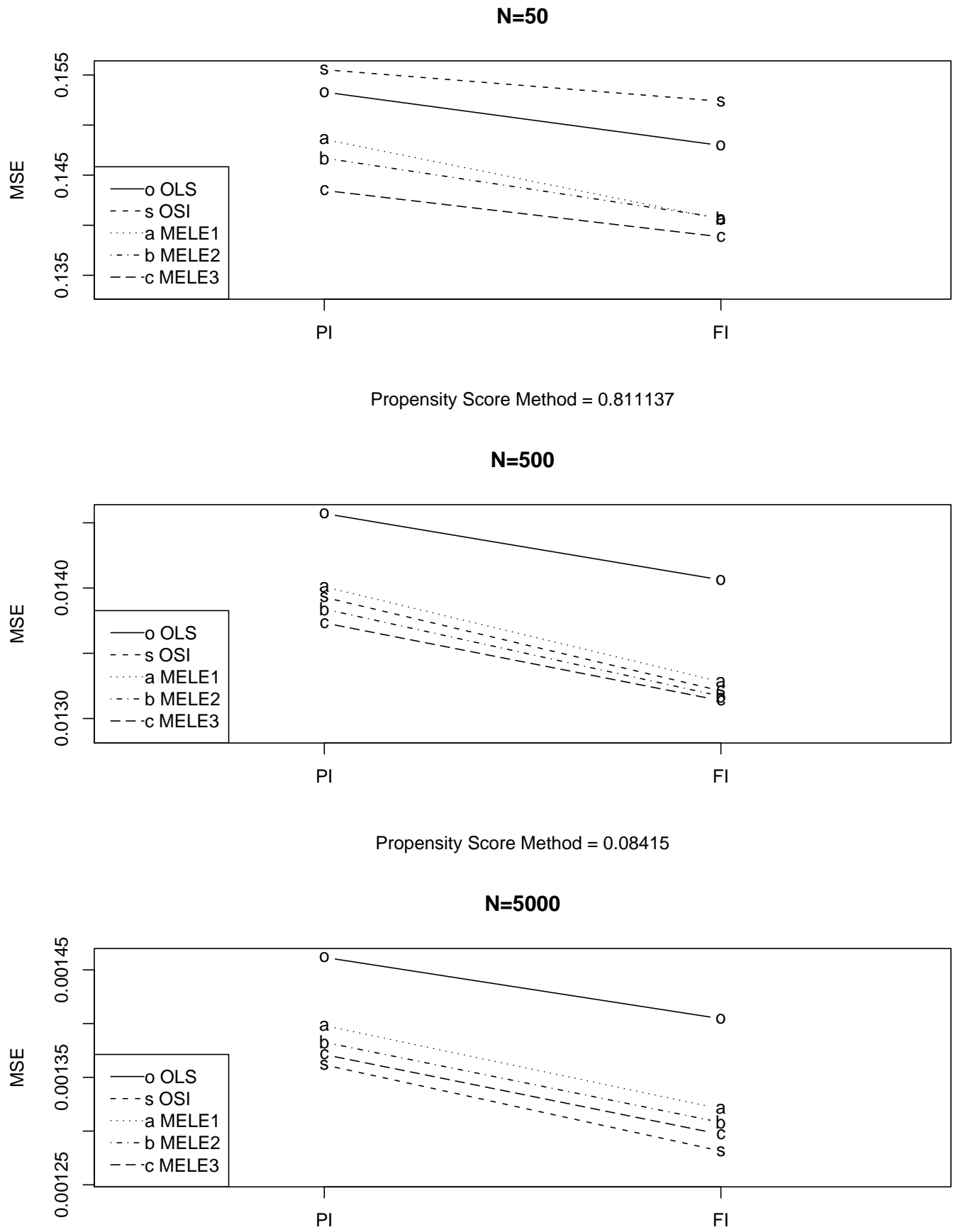

Propensity Score Method $=0.008302$

Fig. 24. MSE for estimating $E[Y]$ where the errors have the Gumbel distribution and an exponential missing structure 
Table XVI. Simulation results showing the MSE for the estimation of $E[Y]$ where the missing structure is exponential and the errors have a normal distribution

\begin{tabular}{|c|c|c|c|c|}
\hline & & $\begin{array}{c}\text { No } \\
\text { Imputation }\end{array}$ & $\begin{array}{c}\text { Partial } \\
\text { Imputation }\end{array}$ & $\begin{array}{c}\text { Full } \\
\text { Imputation }\end{array}$ \\
\hline \multirow{5}{*}{$\mathrm{N}=50$} & OLS & 0.7173 & 0.1135 & 0.1102 \\
\hline & OSI & 0.7173 & 0.1201 & 0.119 \\
\hline & MELE1 & 0.7173 & 0.1145 & 0.1107 \\
\hline & MELE2 & 0.7173 & 0.1145 & 0.1123 \\
\hline & MELE3 & 0.7173 & 0.1128 & 0.1115 \\
\hline \multirow{5}{*}{$\mathrm{N}=500$} & OLS & 0.5884 & 0.01117 & 0.01081 \\
\hline & OSI & 0.5884 & 0.01138 & 0.01106 \\
\hline & MELE1 & 0.5884 & 0.01129 & 0.01093 \\
\hline & MELE2 & 0.5884 & 0.0113 & 0.01098 \\
\hline & MELE3 & 0.5884 & 0.01124 & 0.01095 \\
\hline \multirow{5}{*}{$\mathrm{N}=5000$} & OLS & 0.5772 & 0.001118 & 0.001089 \\
\hline & OSI & 0.5772 & 0.001126 & 0.0011 \\
\hline & MELE1 & 0.5772 & 0.001128 & 0.0011 \\
\hline & MELE2 & 0.5772 & 0.001128 & 0.0011 \\
\hline & MELE3 & 0.5772 & 0.001122 & 0.001096 \\
\hline
\end{tabular}


Table XVII. Simulation results showing the MSE for the estimation of $E[Y]$ where the missing structure is exponential and the errors have a $t_{2}$ distribution

\begin{tabular}{|c|c|c|c|c|}
\hline & & $\begin{array}{c}\text { No } \\
\text { Imputation }\end{array}$ & $\begin{array}{c}\text { Partial } \\
\text { Imputation }\end{array}$ & $\begin{array}{c}\text { Full } \\
\text { Imputation }\end{array}$ \\
\hline \multirow{5}{*}{$\mathrm{N}=50$} & OLS & 1.115 & 0.6219 & 0.5781 \\
\hline & OSI & 1.115 & 0.4435 & 0.3657 \\
\hline & MELE1 & 1.115 & 0.3576 & 0.2465 \\
\hline & MELE2 & 1.115 & 0.3462 & 0.2396 \\
\hline & MELE3 & 1.115 & 0.3444 & 0.2448 \\
\hline \multirow{5}{*}{$\mathrm{N}=500$} & OLS & 0.6689 & 0.09887 & 0.08646 \\
\hline & OSI & 0.6689 & 0.05422 & 0.0325 \\
\hline & MELE1 & 0.6689 & 0.05122 & 0.02831 \\
\hline & MELE2 & 0.6689 & 0.05156 & 0.02843 \\
\hline & MELE3 & 0.6689 & 0.05148 & 0.02917 \\
\hline \multirow{5}{*}{$\mathrm{N}=5000$} & OLS & 0.5844 & 0.009699 & 0.00919 \\
\hline & OSI & 0.5844 & 0.0041 & 0.002932 \\
\hline & MELE1 & 0.5844 & 0.004163 & 0.002518 \\
\hline & MELE2 & 0.5844 & 0.004074 & 0.002474 \\
\hline & MELE3 & 0.5844 & 0.004043 & 0.002449 \\
\hline
\end{tabular}


Table XVIII. Simulation results showing the MSE for the estimation of $E[Y]$ where the missing structure is exponential and the errors have a logistic distribution

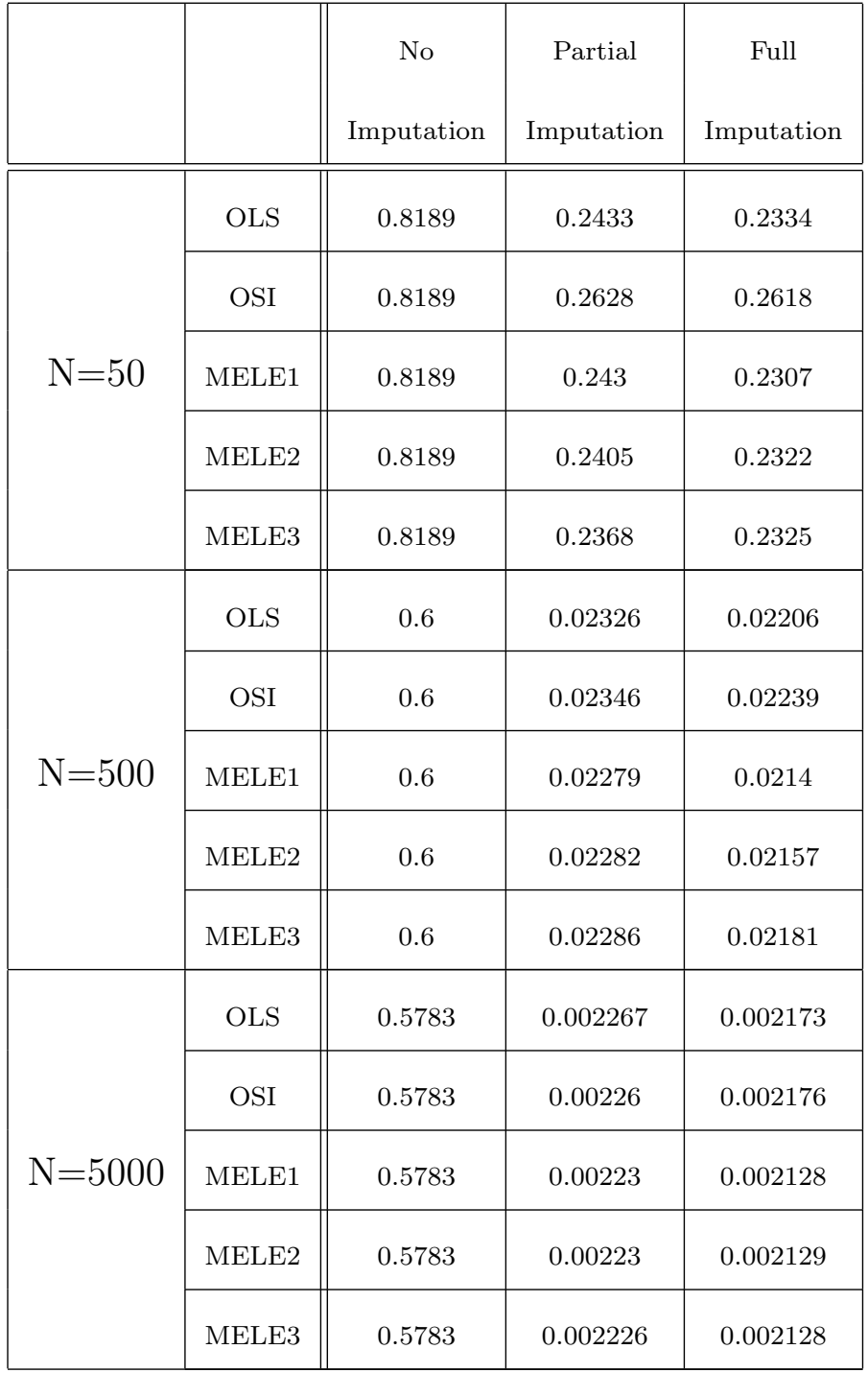


Table XIX. Simulation results showing the MSE for the estimation of $E[Y]$ where the missing structure is exponential and the errors have a Gumbel distribution

\begin{tabular}{|c|c|c|c|c|}
\hline & & $\begin{array}{c}\text { No } \\
\text { Imputation }\end{array}$ & $\begin{array}{c}\text { Partial } \\
\text { Imputation }\end{array}$ & $\begin{array}{c}\text { Full } \\
\text { Imputation }\end{array}$ \\
\hline \multirow{5}{*}{$\mathrm{N}=50$} & OLS & 0.7453 & 0.1533 & 0.148 \\
\hline & OSI & 0.7453 & 0.1555 & 0.1524 \\
\hline & MELE1 & 0.7453 & 0.1486 & 0.1406 \\
\hline & MELE2 & 0.7453 & 0.1467 & 0.1407 \\
\hline & MELE3 & 0.7453 & 0.1435 & 0.1388 \\
\hline \multirow{5}{*}{$\mathrm{N}=500$} & OLS & 0.594 & 0.01457 & 0.01406 \\
\hline & OSI & 0.594 & 0.01393 & 0.01321 \\
\hline & MELE1 & 0.594 & 0.01401 & 0.01328 \\
\hline & MELE2 & 0.594 & 0.01384 & 0.01317 \\
\hline & MELE3 & 0.594 & 0.01373 & 0.01314 \\
\hline \multirow{5}{*}{$\mathrm{N}=5000$} & OLS & 0.5778 & 0.001462 & 0.001405 \\
\hline & OSI & 0.5778 & 0.001362 & 0.001281 \\
\hline & MELE1 & 0.5778 & 0.001398 & 0.001321 \\
\hline & MELE2 & 0.5778 & 0.001383 & 0.001308 \\
\hline & MELE3 & 0.5778 & 0.001371 & 0.001297 \\
\hline
\end{tabular}


Table XX. Simulation results showing the MSE for the estimation of $E[Y]$ where the missing structure is exponential and the errors have a gamma distribution

\begin{tabular}{|c|c|c|c|c|}
\hline & & $\begin{array}{c}\text { No } \\
\text { Imputation }\end{array}$ & $\begin{array}{c}\text { Partial } \\
\text { Imputation }\end{array}$ & $\begin{array}{c}\text { Full } \\
\text { Imputation }\end{array}$ \\
\hline \multirow{5}{*}{$\mathrm{N}=50$} & OLS & 0.7589 & 0.1702 & 0.1639 \\
\hline & OSI & 0.7589 & 0.1629 & 0.1558 \\
\hline & MELE1 & 0.7589 & 0.159 & 0.1478 \\
\hline & MELE2 & 0.7589 & 0.154 & 0.1442 \\
\hline & MELE3 & 0.7589 & 0.1473 & 0.1377 \\
\hline \multirow{5}{*}{$\mathrm{N}=500$} & OLS & 0.5911 & 0.01654 & 0.01588 \\
\hline & OSI & 0.5911 & 0.01423 & 0.01272 \\
\hline & MELE1 & 0.5911 & 0.015 & 0.01375 \\
\hline & MELE2 & 0.5911 & 0.01459 & 0.01343 \\
\hline & MELE3 & 0.5911 & 0.01418 & 0.013 \\
\hline \multirow{5}{*}{$\mathrm{N}=5000$} & OLS & 0.5776 & 0.001632 & 0.001567 \\
\hline & OSI & 0.5776 & 0.001299 & 0.001136 \\
\hline & MELE1 & 0.5776 & 0.00141 & 0.001262 \\
\hline & MELE2 & 0.5776 & 0.001399 & 0.001269 \\
\hline & MELE3 & 0.5776 & 0.001372 & 0.001244 \\
\hline
\end{tabular}


B. $\mathrm{R}$ code

1. Simulations with calculation of $\vartheta$ and $\mathrm{E}(\mathrm{Y})$

The variable filenum changes which sample size, missingness structure and error distribution is used. The final result creates a different file for each filenum.

for (filenum in $1: 45)\{$

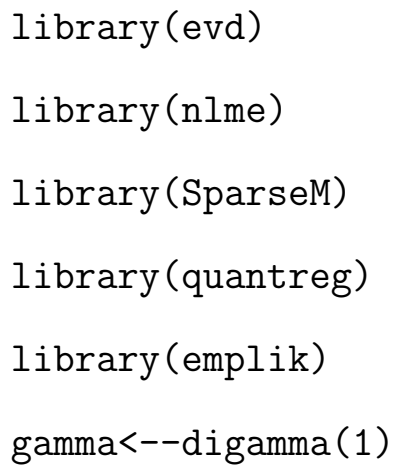




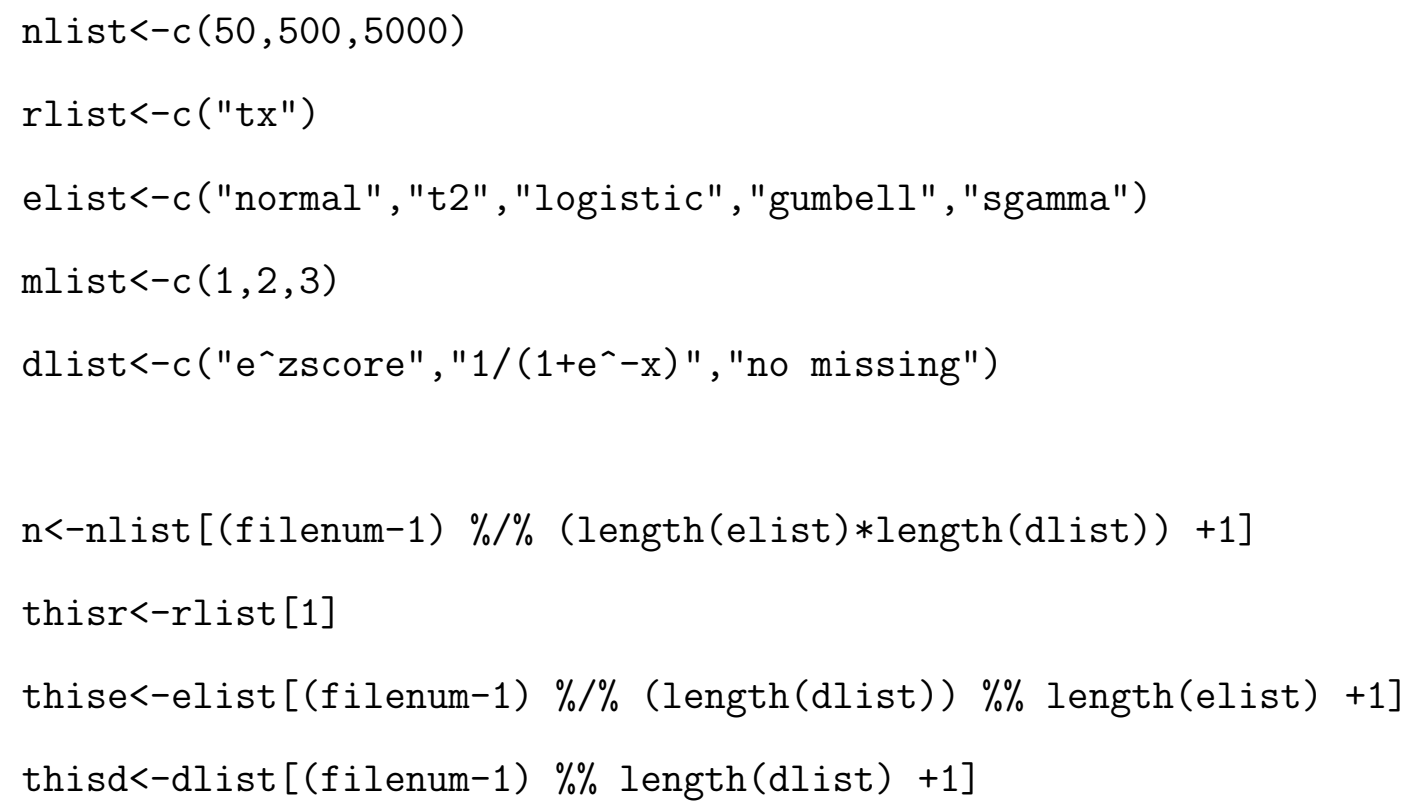

\#\#Define theta, r (parametric pieces) \#\#CHANGE PARAMETER ESTIMATION IF YOU CHANGE THE MODEL FORM 


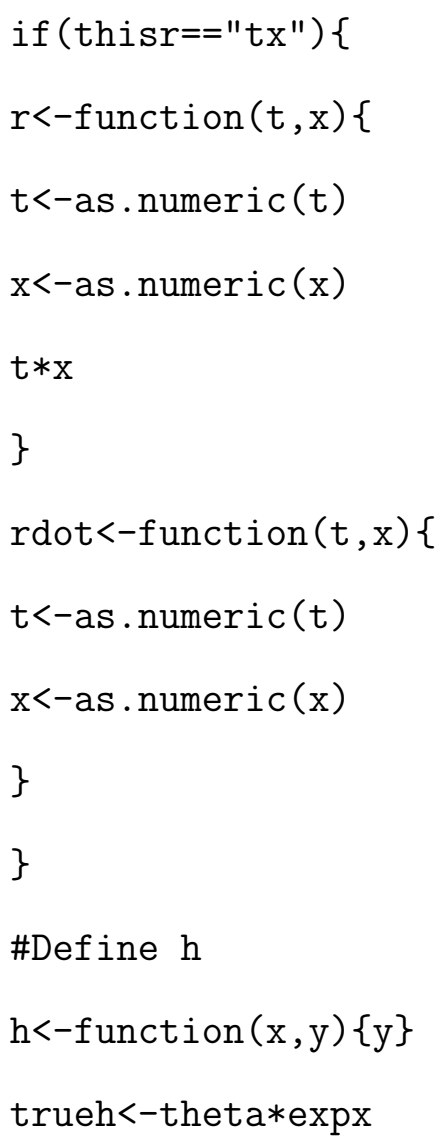




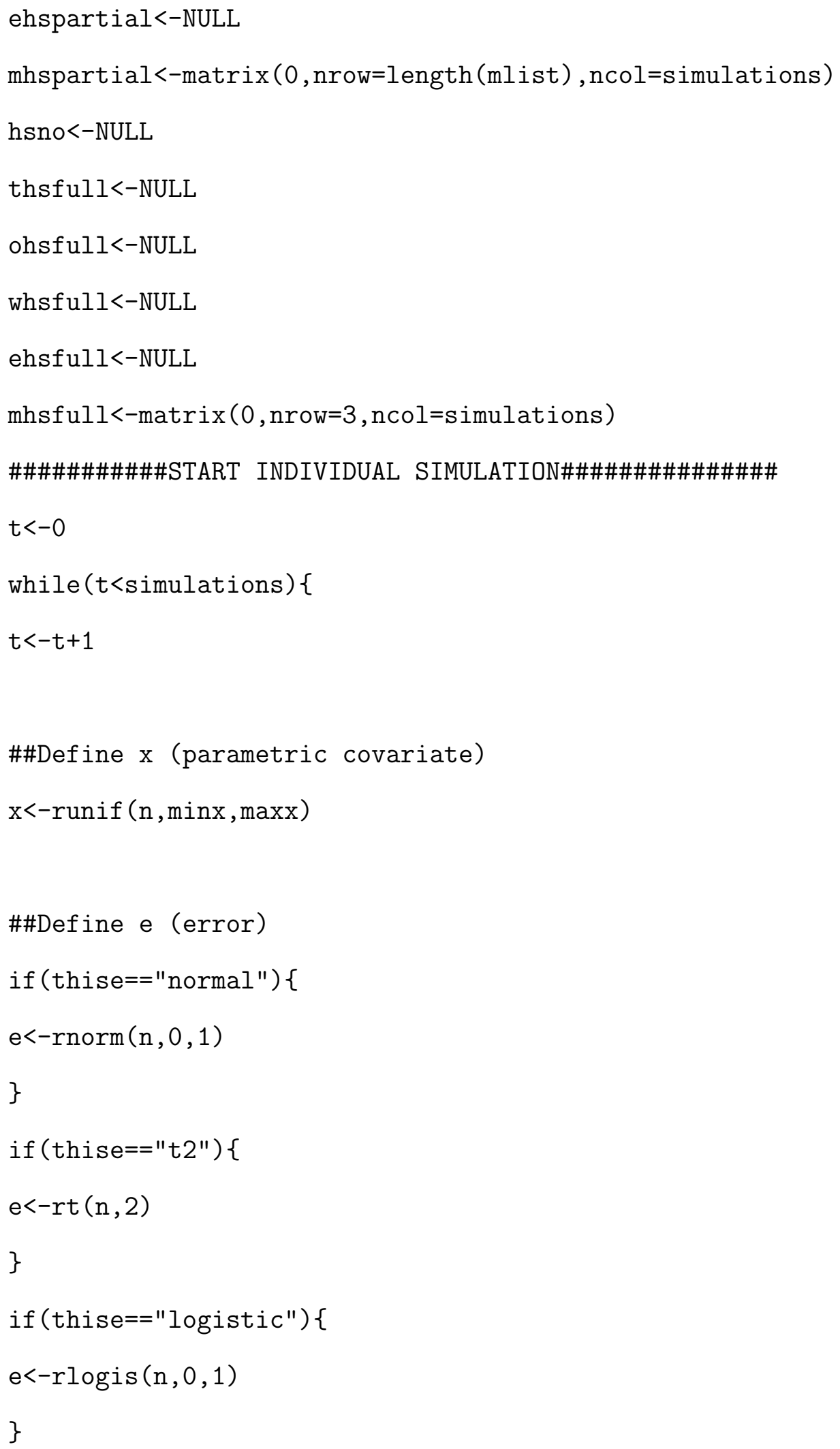




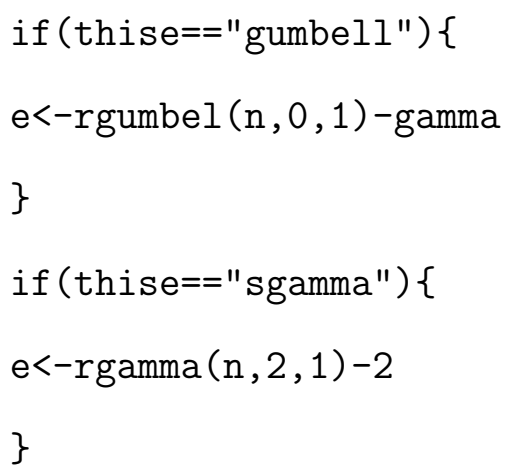




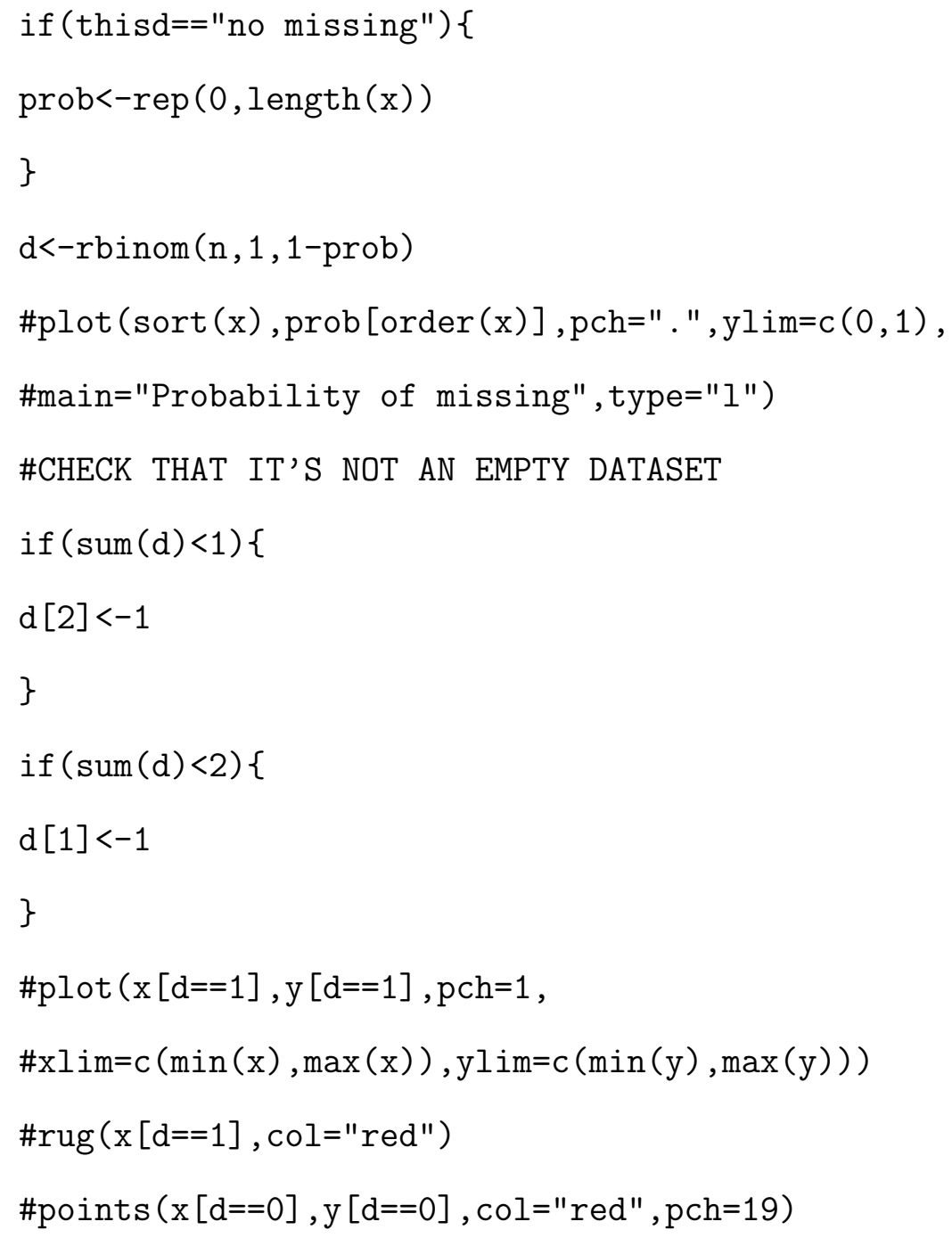

\#TRUE

\#theta<-theta

\#OLSE

othetas $[t]<-\operatorname{lm}(y[d==1] \sim 0+x[d==1])$ \$coefficients $[1]$

\#WLSE 


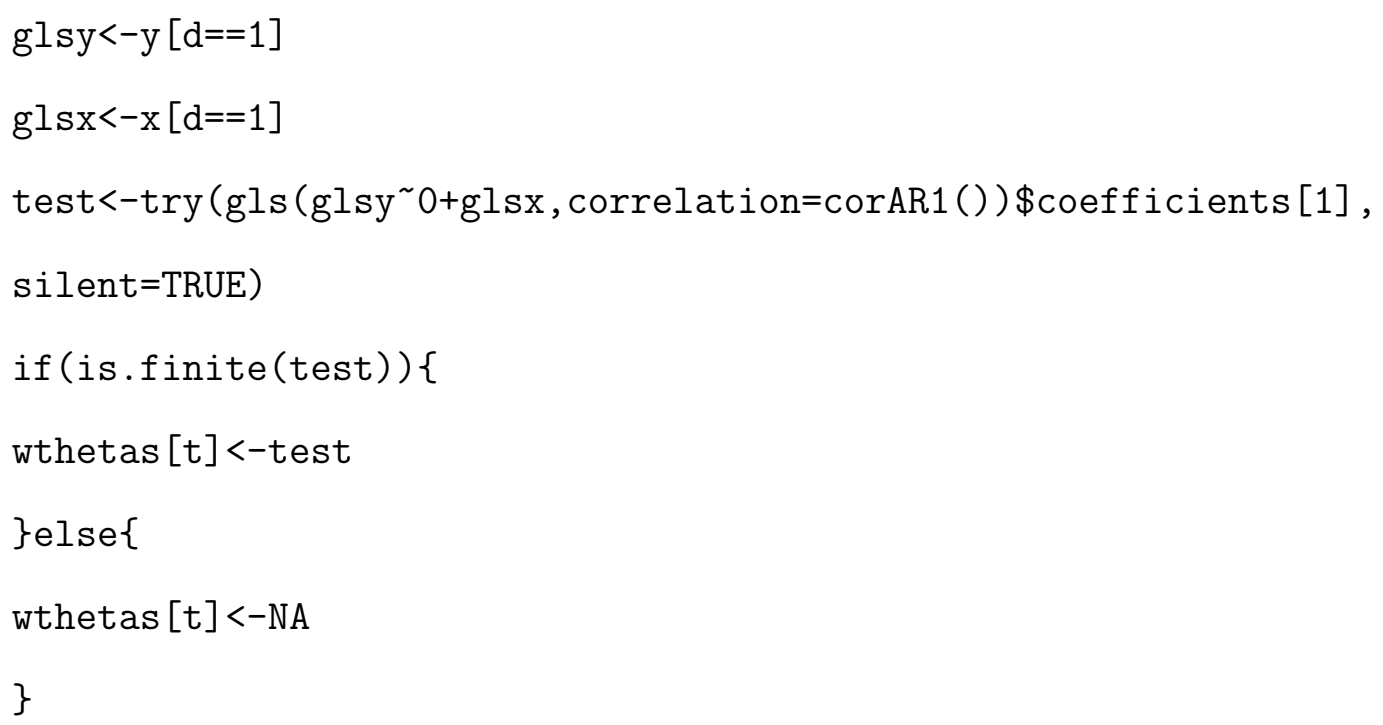




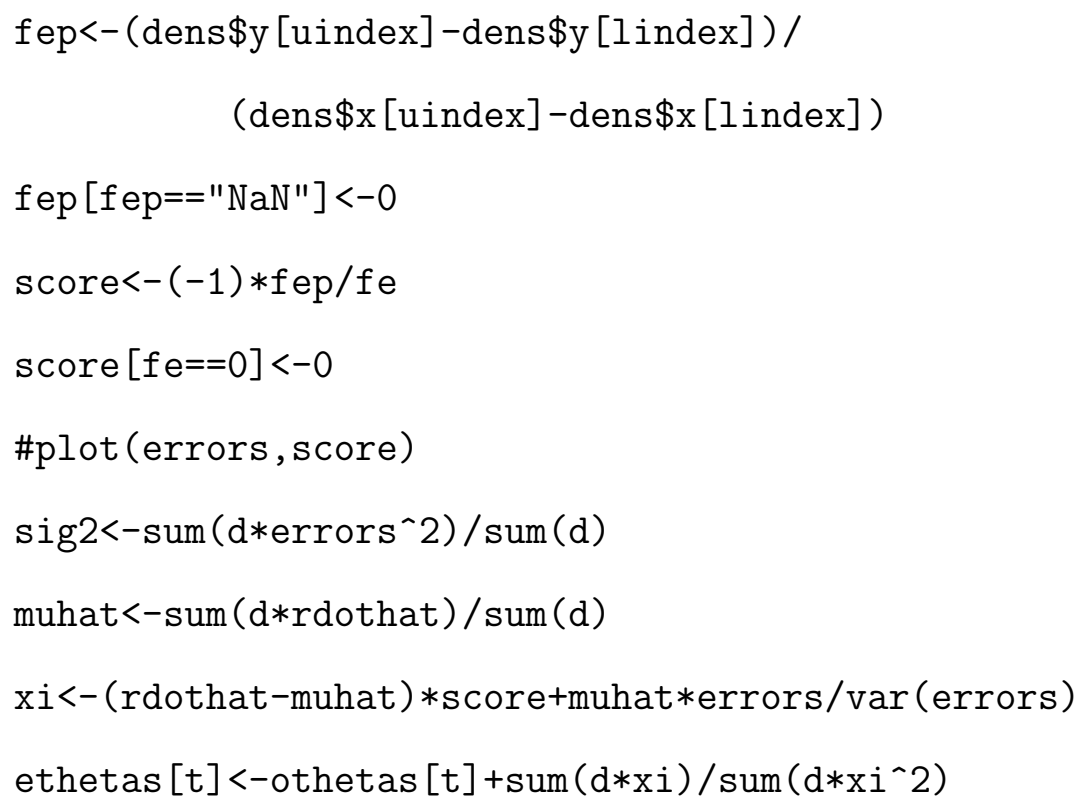

\section{\#MELE}

$\mathrm{fbi}<-\mathrm{NULL}$

$\mathrm{nn}<-\operatorname{sum}(\mathrm{d}==1)$

\#nb defined at top

bmin<-min (theta, ethetas [t]) -abs (ethetas [t]-theta)*. 5

$\operatorname{bmax}<-\max ($ theta, ethetas $[\mathrm{t}])+\operatorname{abs}($ ethetas $[\mathrm{t}]-$ theta $) * .5$

testb<-seq (bmin, bmax, length=nb)

likes<-matrix (1000, length (mlist), nb)

for $(b$ in $1: n b)\{$

$z i<-y[d==1]-$ testb $[b] * x[d==1]$

$\mathrm{fbi}<-N U L L$

for (ni in $1: n n)$

fbi<-c (fbi, 1/nn*sum (zi [ni] <=zi))

for (m in $1:$ length(mlist) $)\{$

$\operatorname{phik}<-\operatorname{sqrt}(2) * \cos (\operatorname{rep}(\operatorname{seq}(1, m), \operatorname{each}=n n) * \operatorname{pi} * f b i)$ 


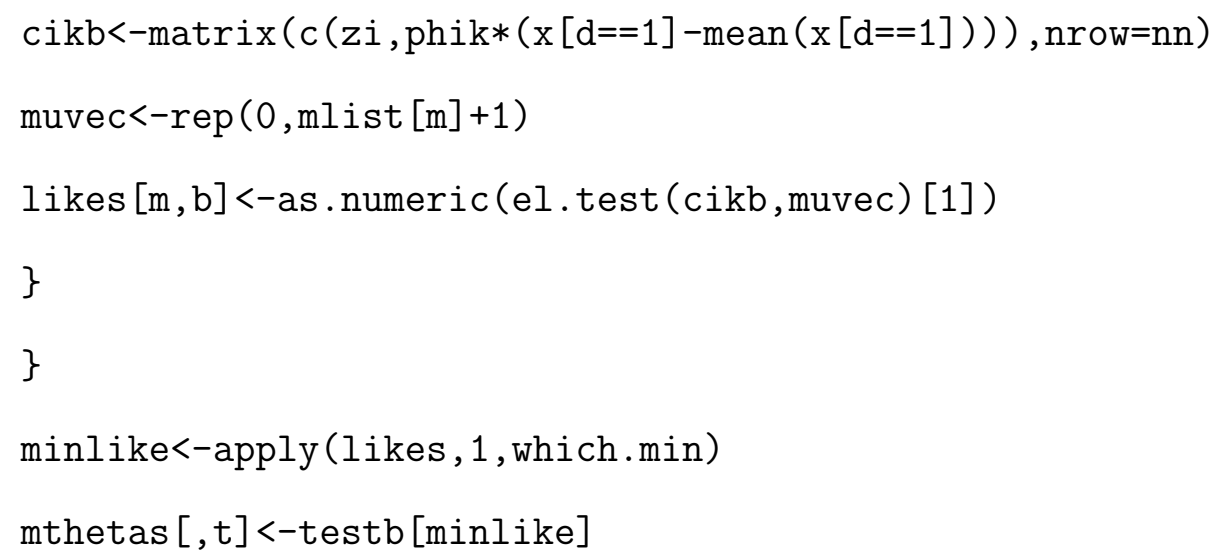




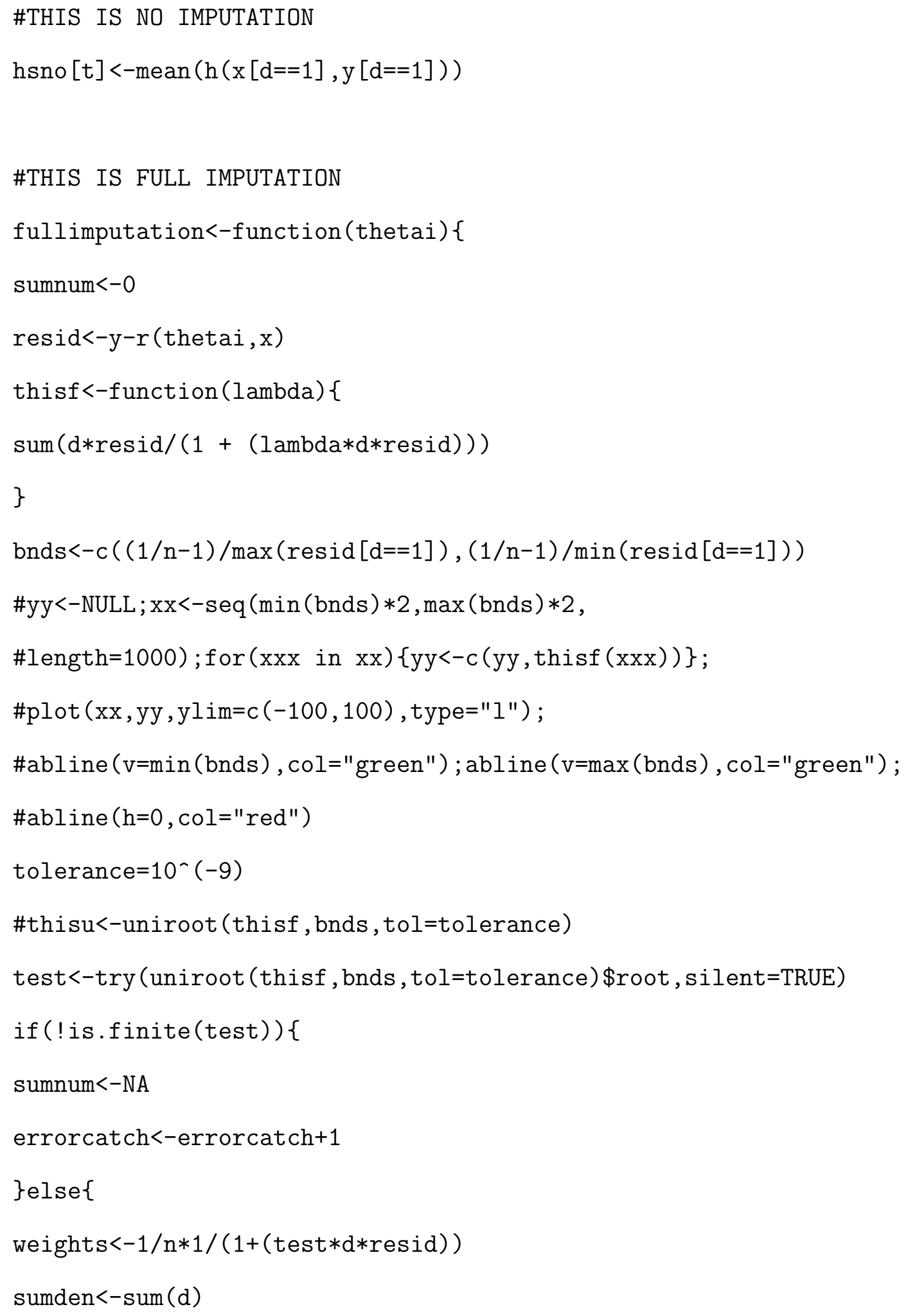




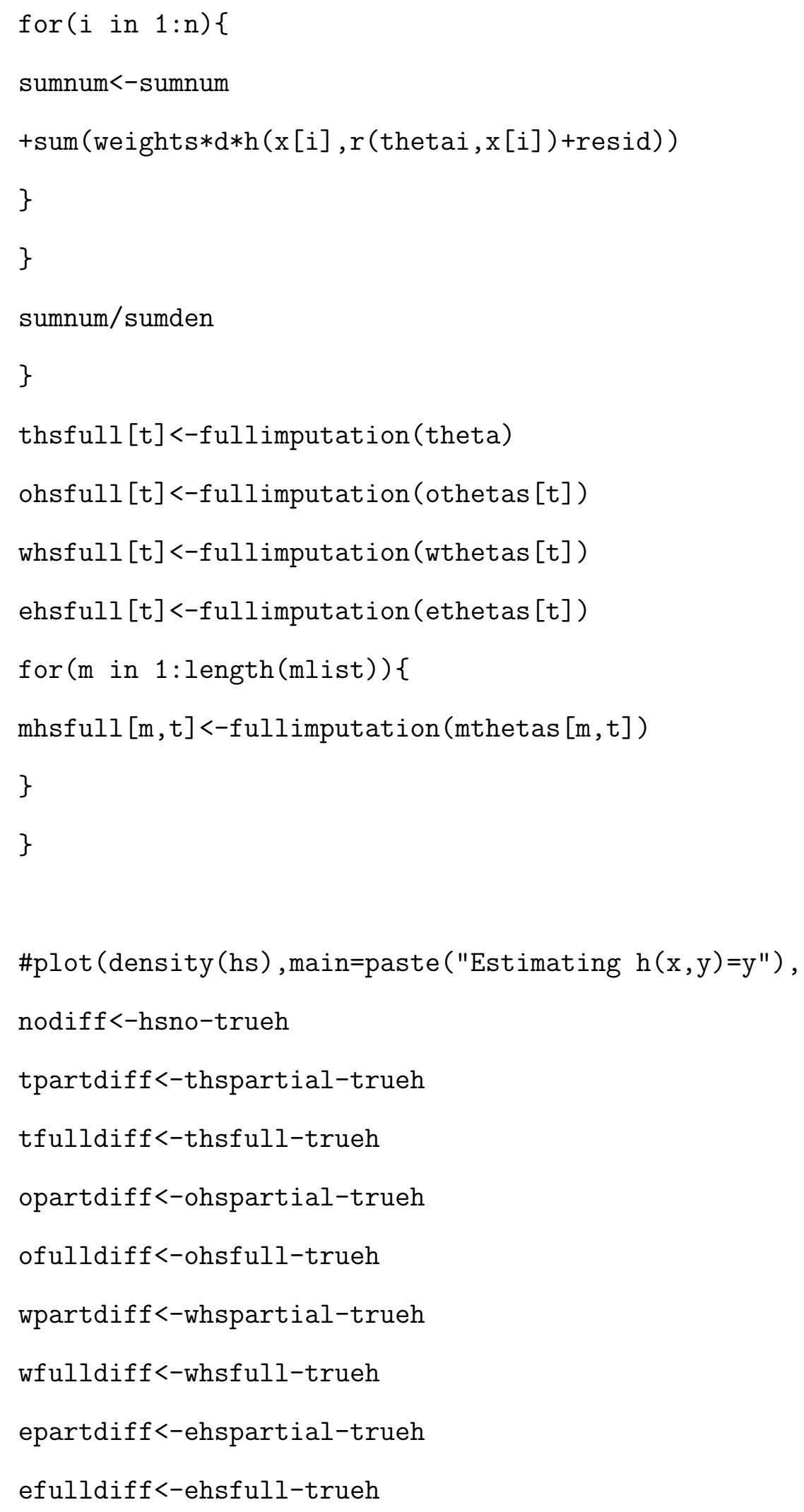




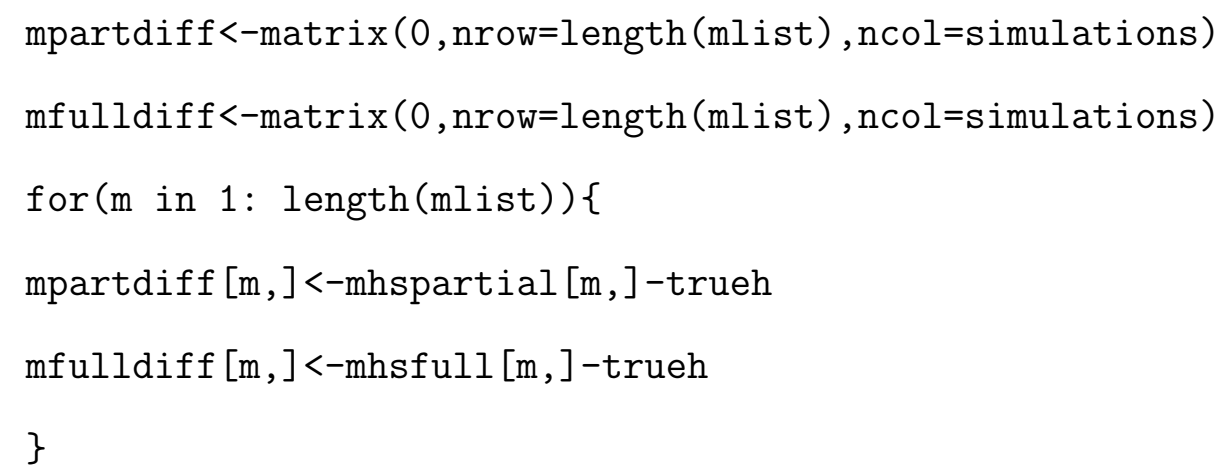




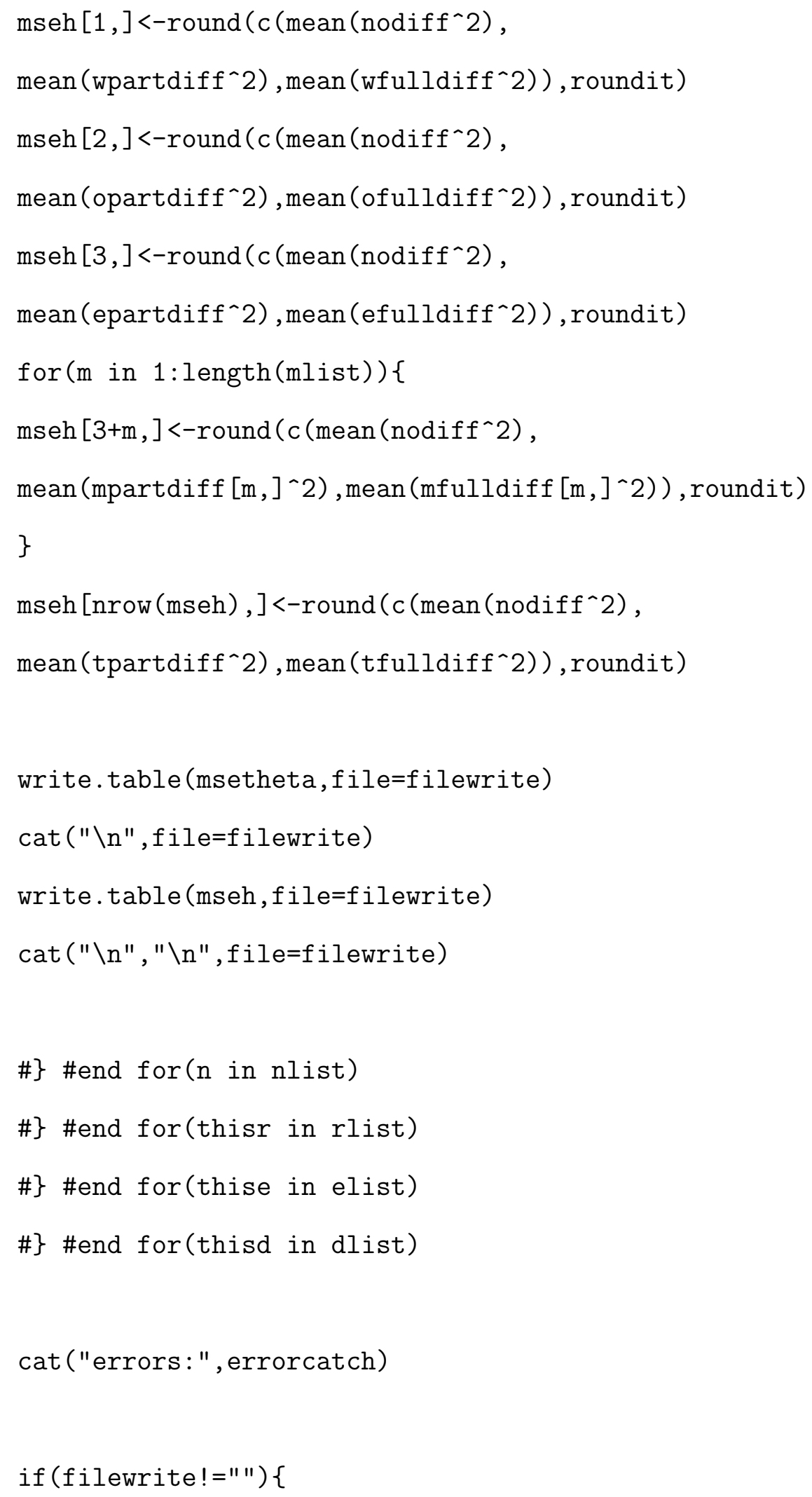


2. Combine output files

$R$-code used to take the multiple output data files and combine them together to find the average across all simulations. Some simulations were lost due to errors, and those are dropped and accounted for. The final output is a matrix called "MSEEY" which has for each row a scenario (a different combination of error structure, missingness structure, and sample size) and for each column a different estimate for $\vartheta$ (WLS, OLS, etc).

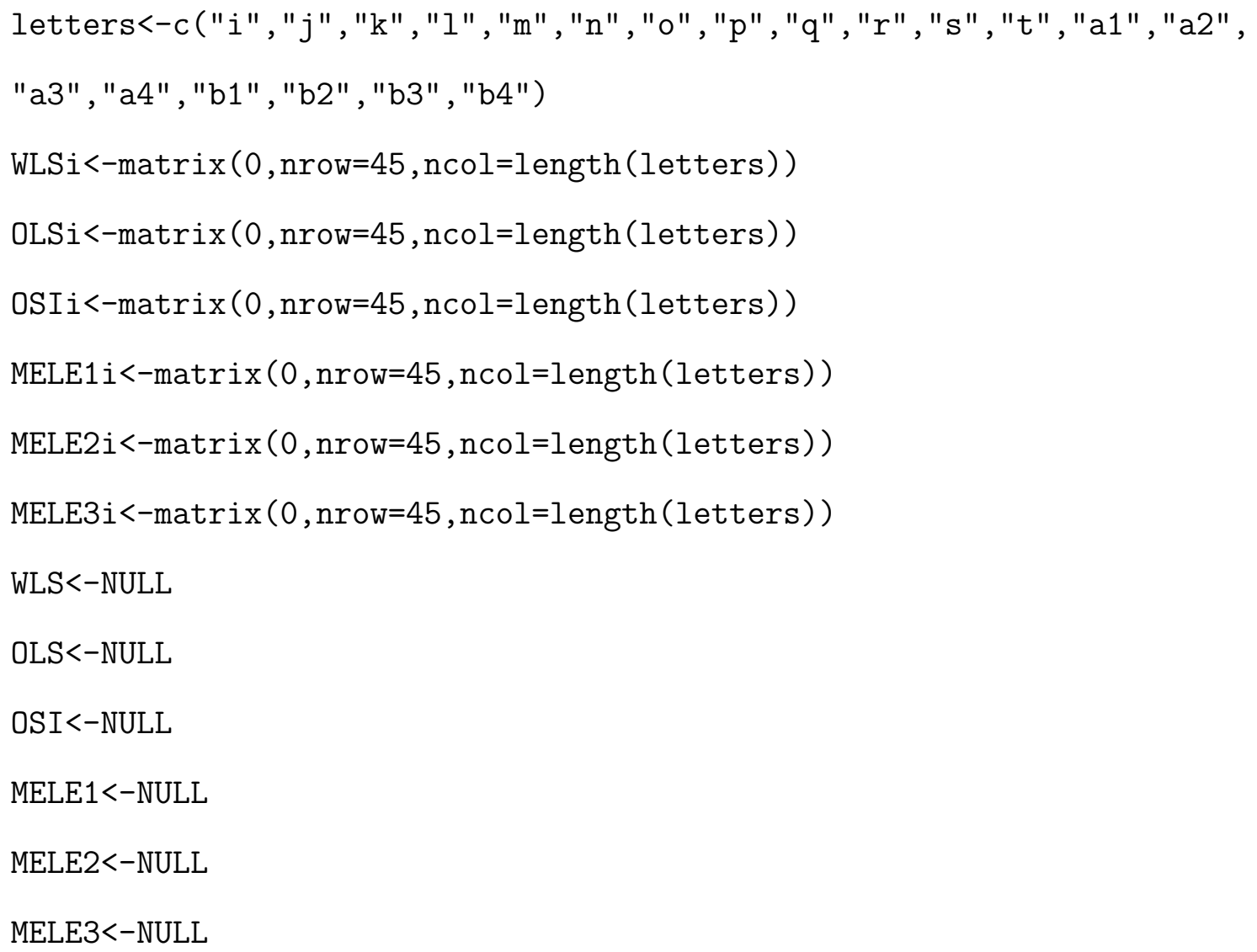




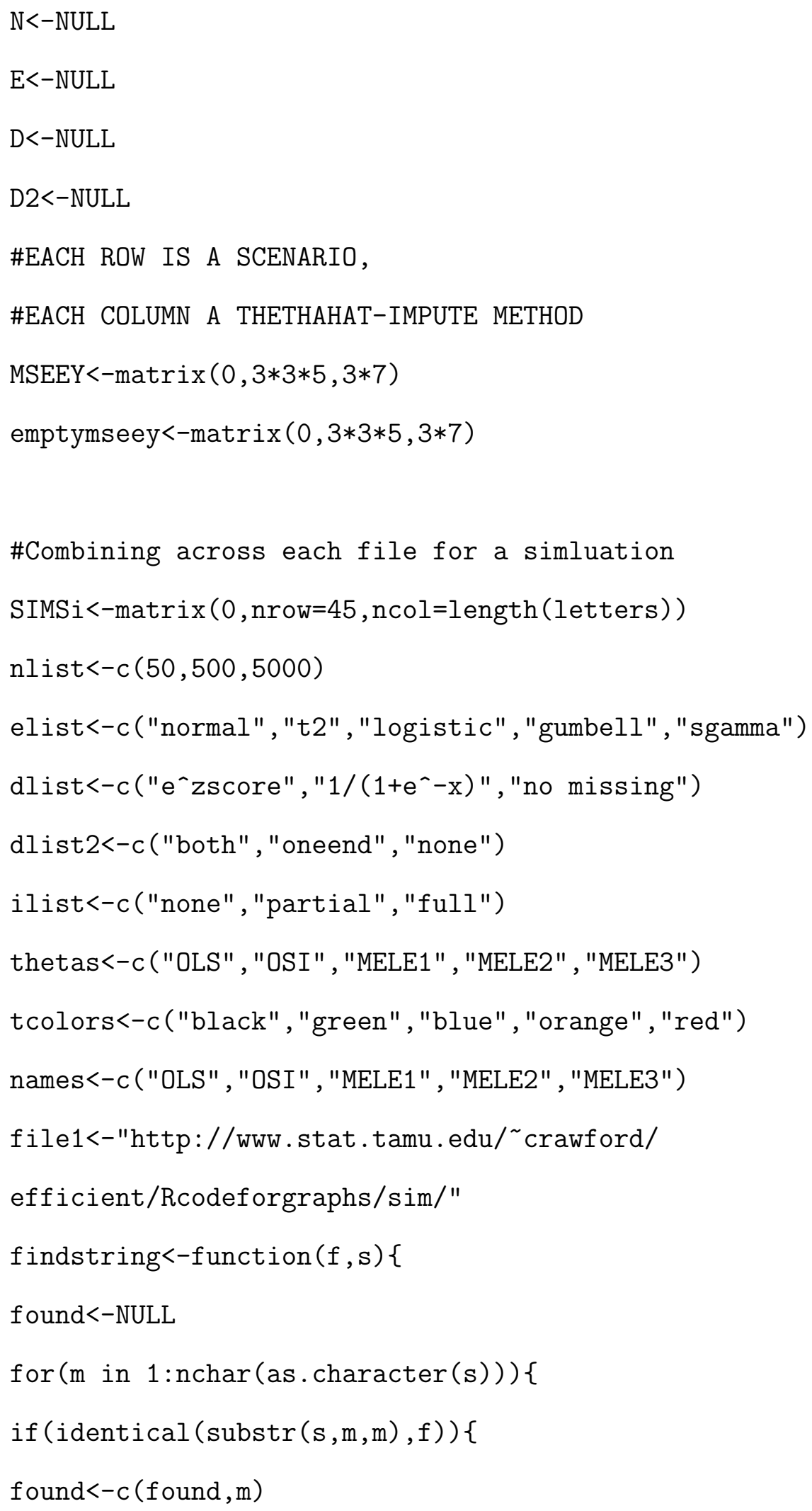




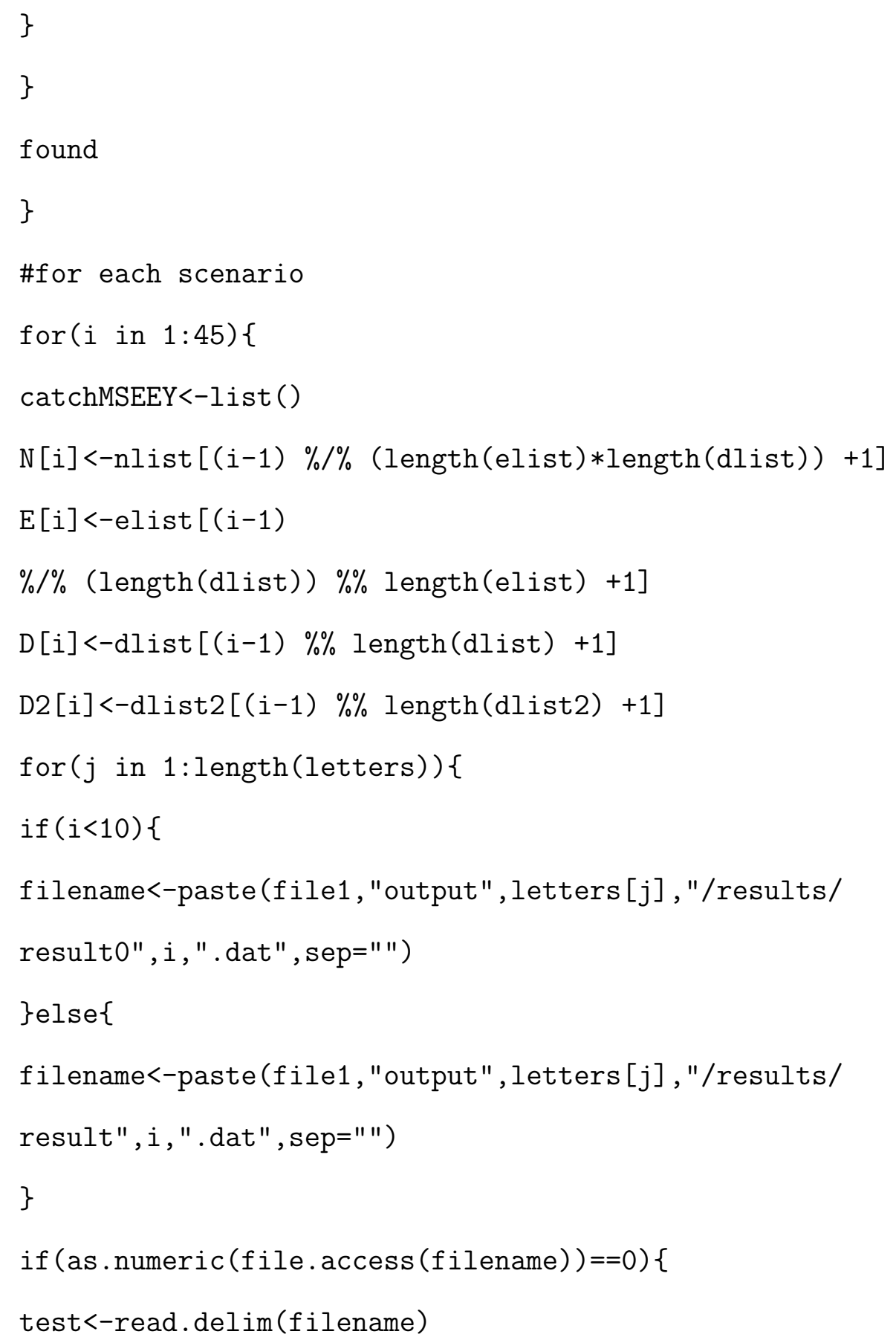




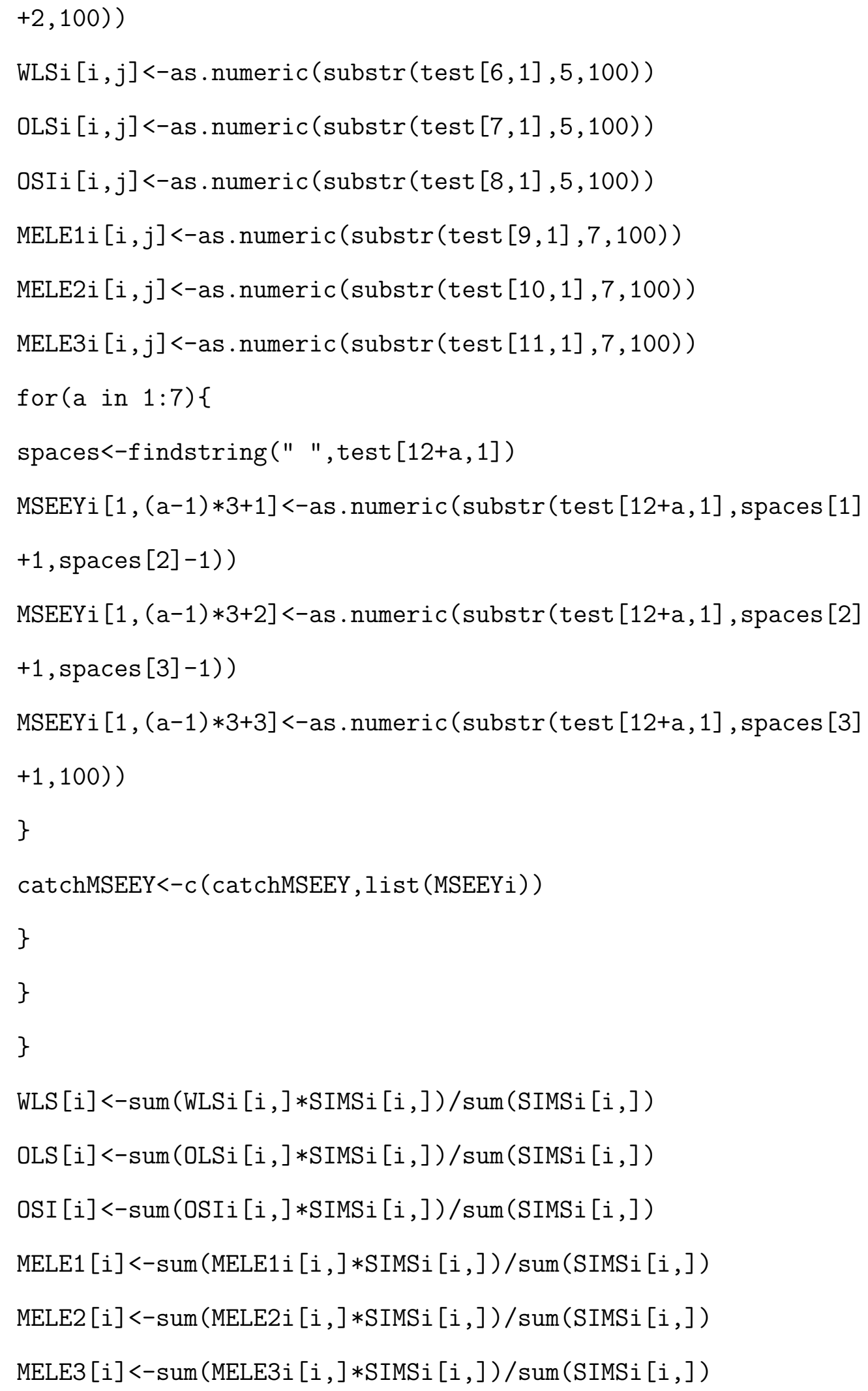




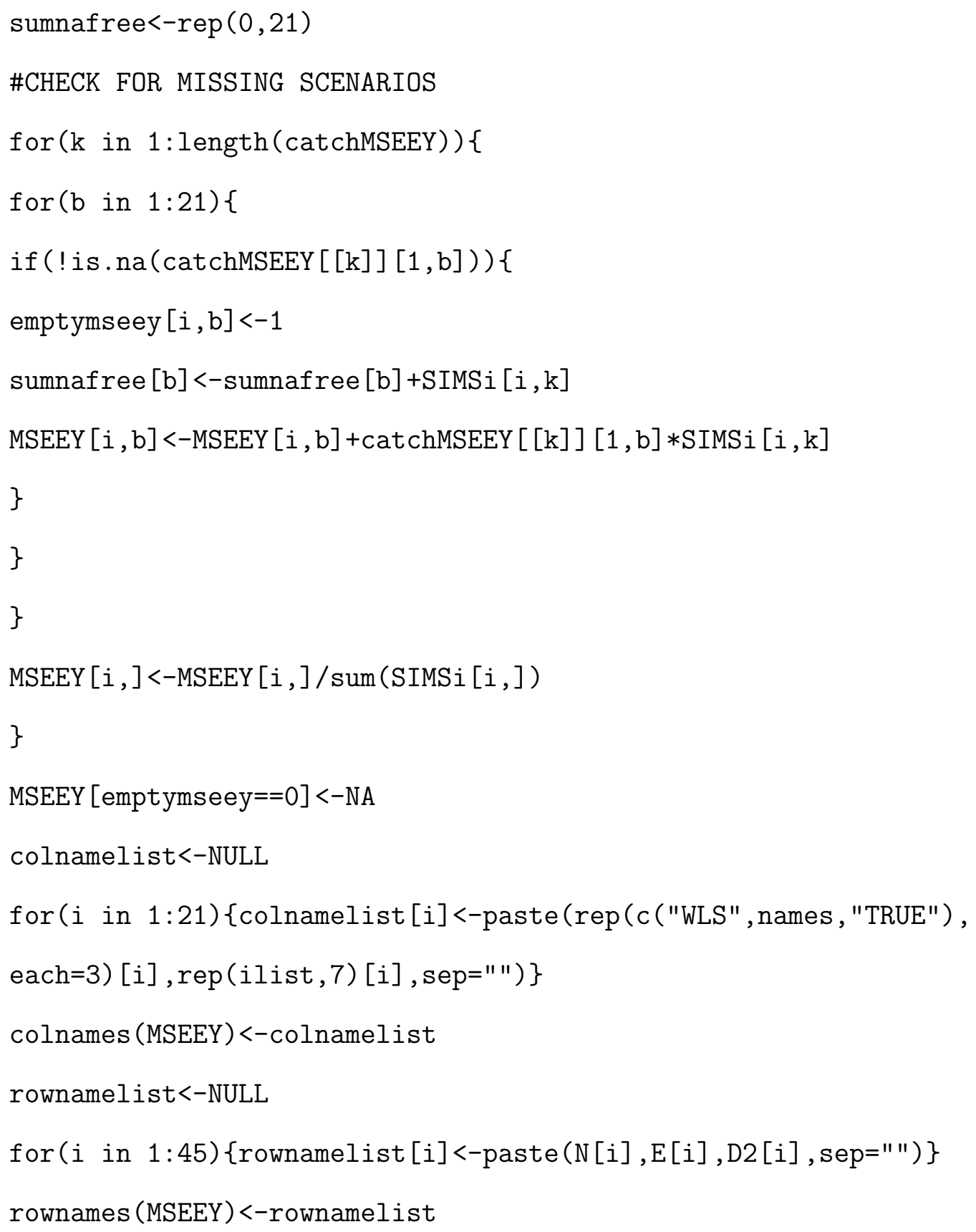




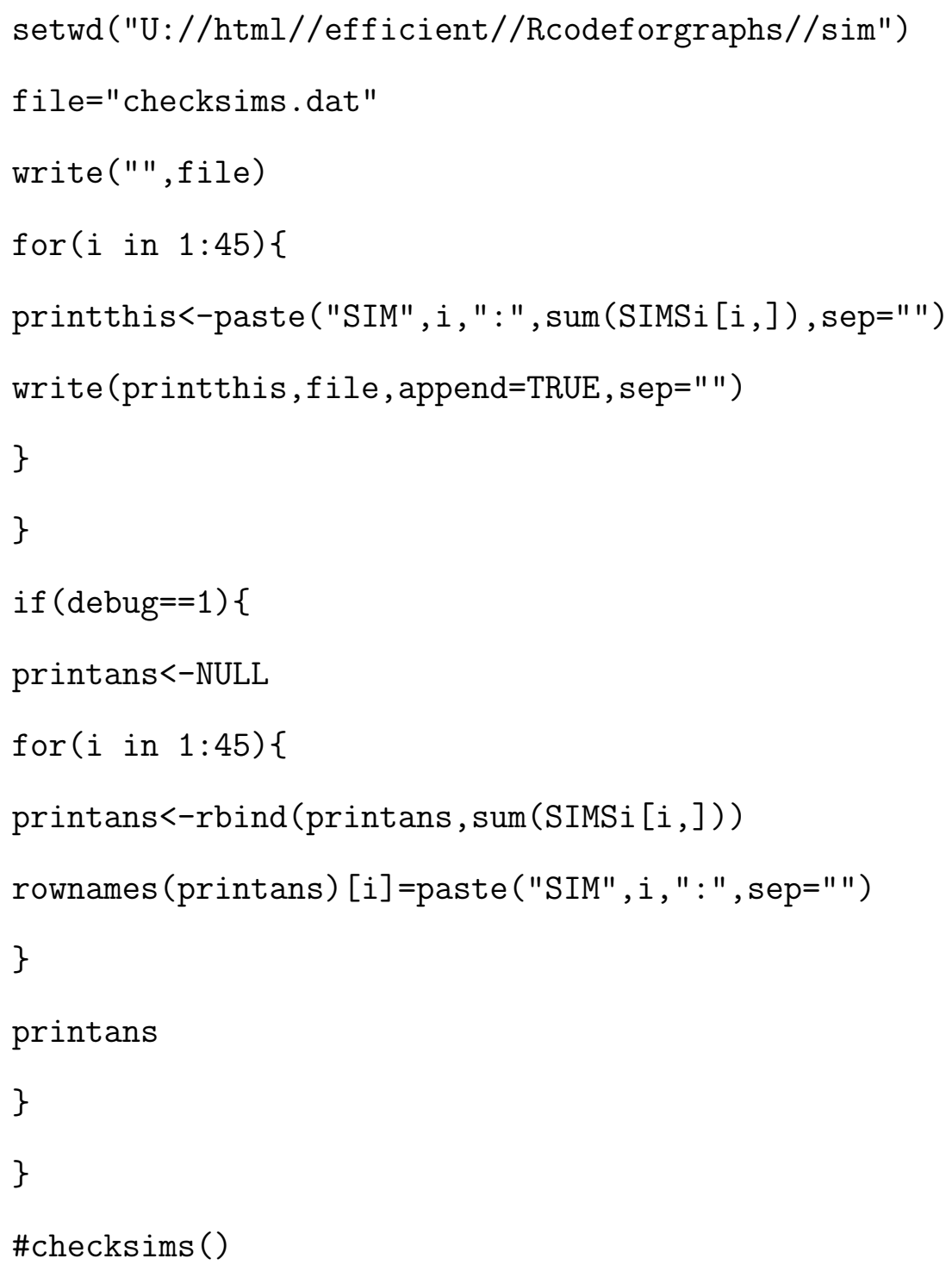

3. Graph the MSE for the estimation of $\vartheta$

The "plotj" function does the plot for scenario $\mathrm{j}$ where each scenario is a unique sample size, missingness structure and error distribution combination.

\#FUNCTION TO FIT A SMOOTH 1/MSE CURVE

fitoox $<-$ function $(x, y)\{$

$o x<-1 / x$ 


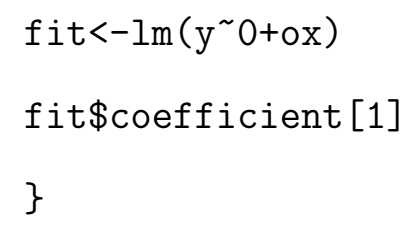




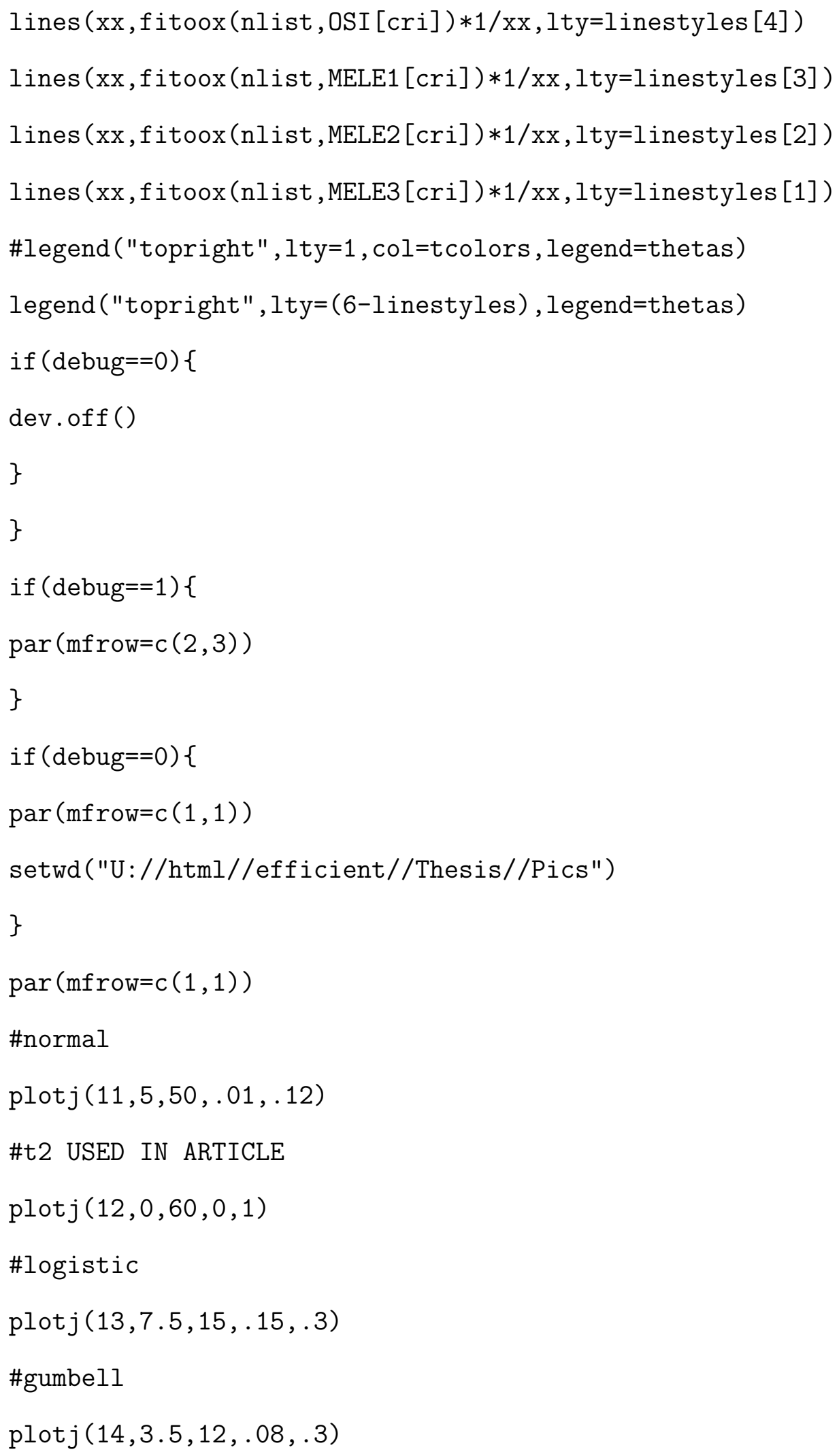




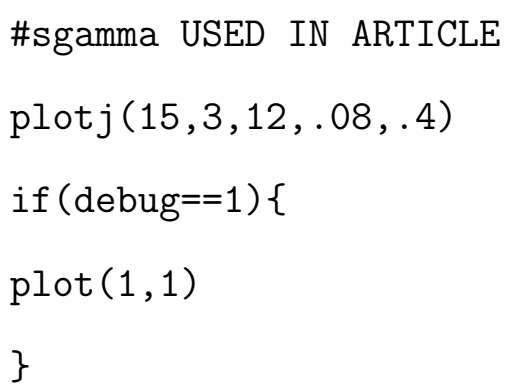

4. Create a table for the estimation of $\vartheta$

Final result prints out the table in Latex code.

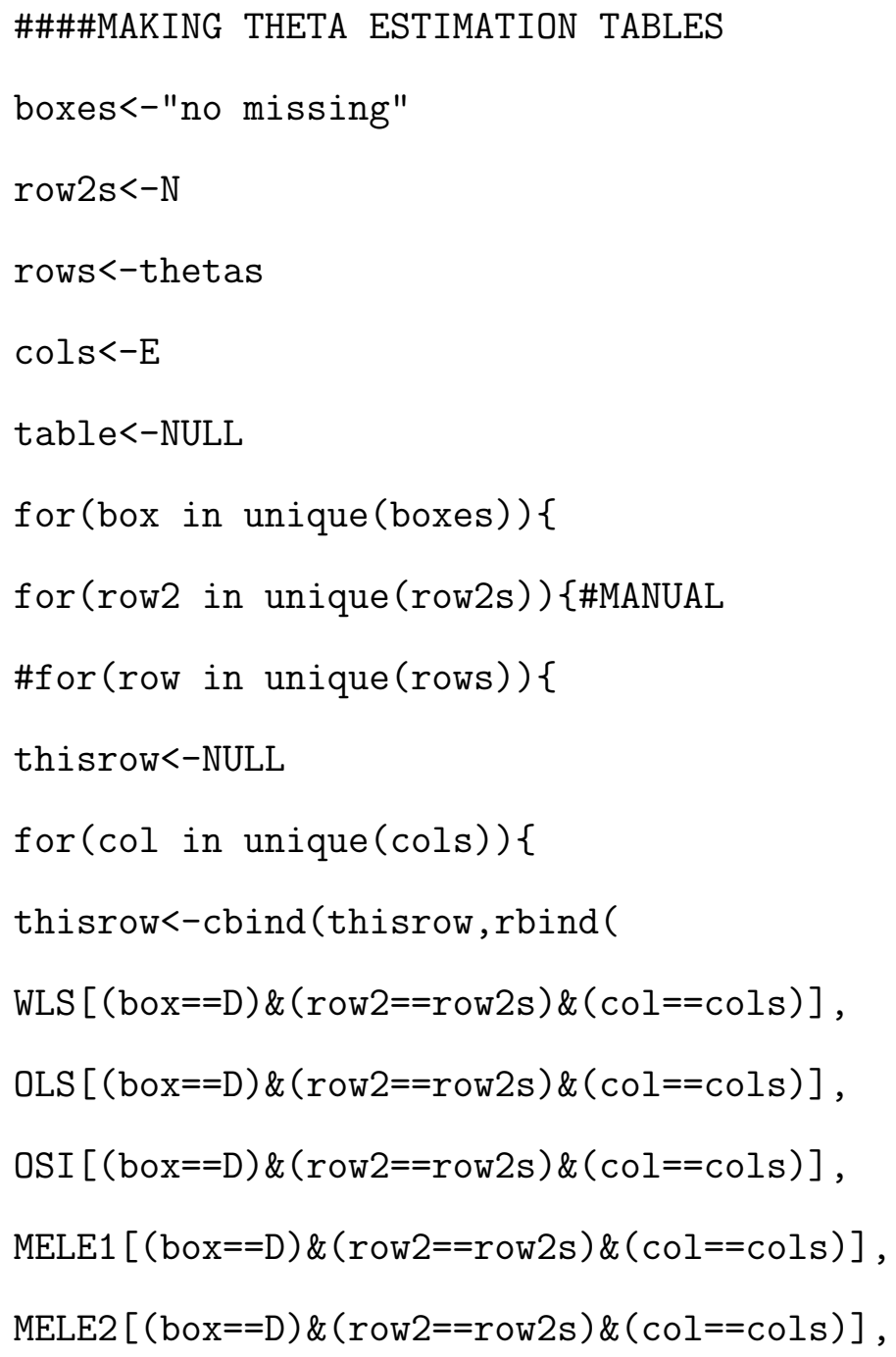




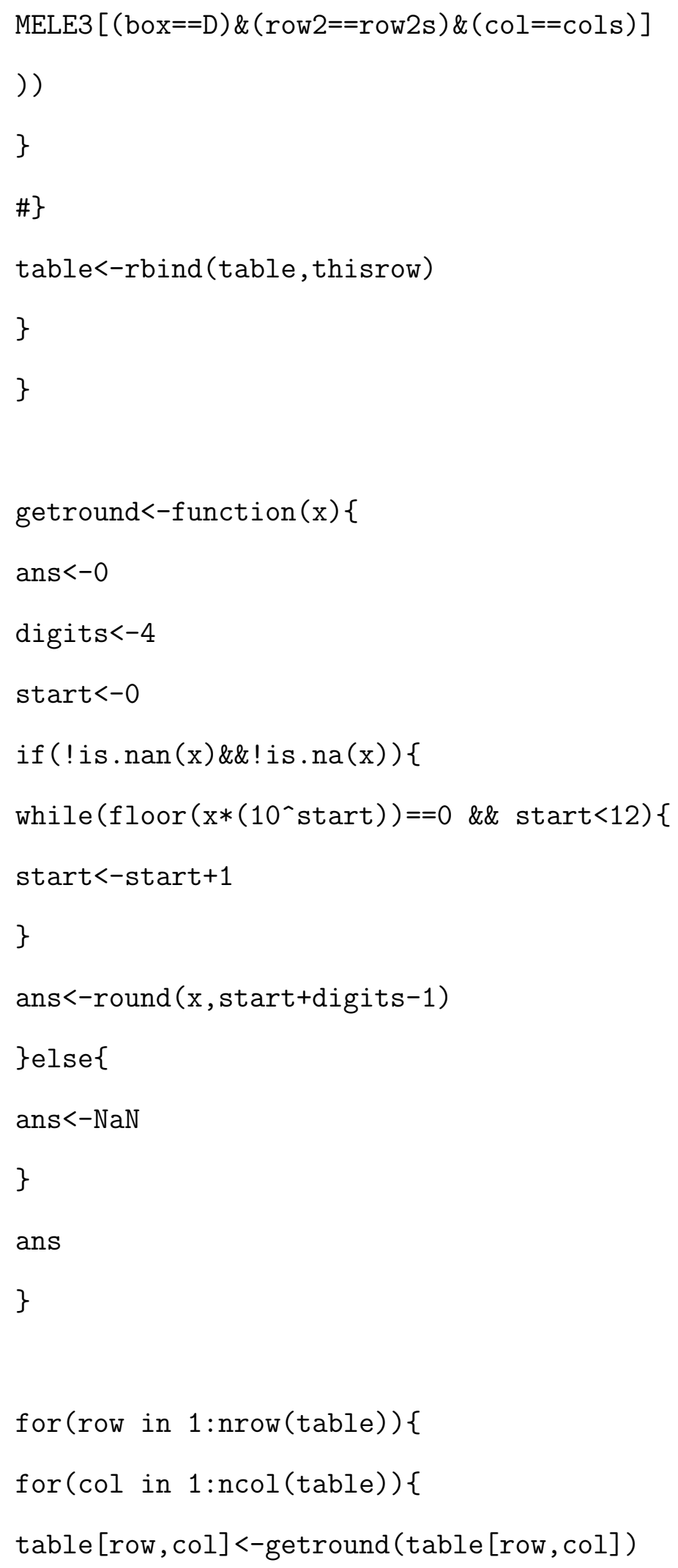




\section{\} \\ \}}

if $($ debug $==0)\{$

setwd ("U://html//efficient//Thesis//Thesispieces")

file="App-simtable.tex"

write(" ",file)

write("\\clearpage", file, append=TRUE)

write("\\appendix\{Tables showing the results

from the simulation\}",file, append=TRUE)

write("\\label\{app:tab\}", file, append=TRUE)

for (row in 1 :nrow (table)) \{

if (row

$\% \%($ length (unique (rows) $) *$ length $($ unique $($ row $2 s)))==1)\{$

write("\n\n", file, append=TRUE)

write("\\begin\{table\}",file, append=TRUE) }

write("\\begin } \{ \text { center } \} " \text { , file, append=TRUE) }

capinput<-" "

capinput<-paste (capinput,

"\\caption\{Simulation results for the estimation of $\$ \backslash \backslash$ param $\$ "$, sep=" ")

\#if (row

$\% / \%($ length (unique (rows) $) *$ length (unique $($ row $2 \mathrm{~s})))==0)\{$

\#capinput<-paste (capinput,

" where the missing structure is on both ends", sep=" ")

\#capinput<-paste(capinput, 
"\\label\{tab: thetaboth\}", set=" ")

\#\}

\#if (row

$\%($ length $($ unique $($ rows $)) *$ length $($ unique $($ row $2 \mathrm{~s})))==1)\{$

\#capinput<-paste (capinput,

" where the missing structure is on one end", sep="")

\#capinput<-paste (capinput,

"\\label\{tab: thetaone $\} "$, set=" ")

\#\}

\#if (row

$\% / \%($ length $($ unique $($ rows $)) *$ length $($ unique $($ row $2 \mathrm{~s})))==2)\{$

\#capinput<-paste (capinput,

" where there is no missing data", sep="")

capinput<-paste (capinput,

"\\label\{tab: thetano\}", set=" ")

\#\}

capinput<-paste (capinput, "\}", sep=" ")

write (capinput, file, append=TRUE)

write("\\
begin } \{ \text { tabular } \} \{ | c | c | | c | c | c | c | c | \} " \text { , file, append=TRUE) }

write("\\hline",file, append=TRUE)

write ("\&\&Normal\&\$t_2\$\&Logistic\&Gumbel\&Gamma $\backslash \backslash \backslash "$,

file, append=TRUE)

\#write ("\\vspace $\{-20 p t\} "$, file, append=TRUE)

write ("\&\&errors\&errors\&errors\&errors\&errors $\backslash \backslash \backslash \backslash "$,

file, append=TRUE)

write("\\hline \\hline",file, append=TRUE) 


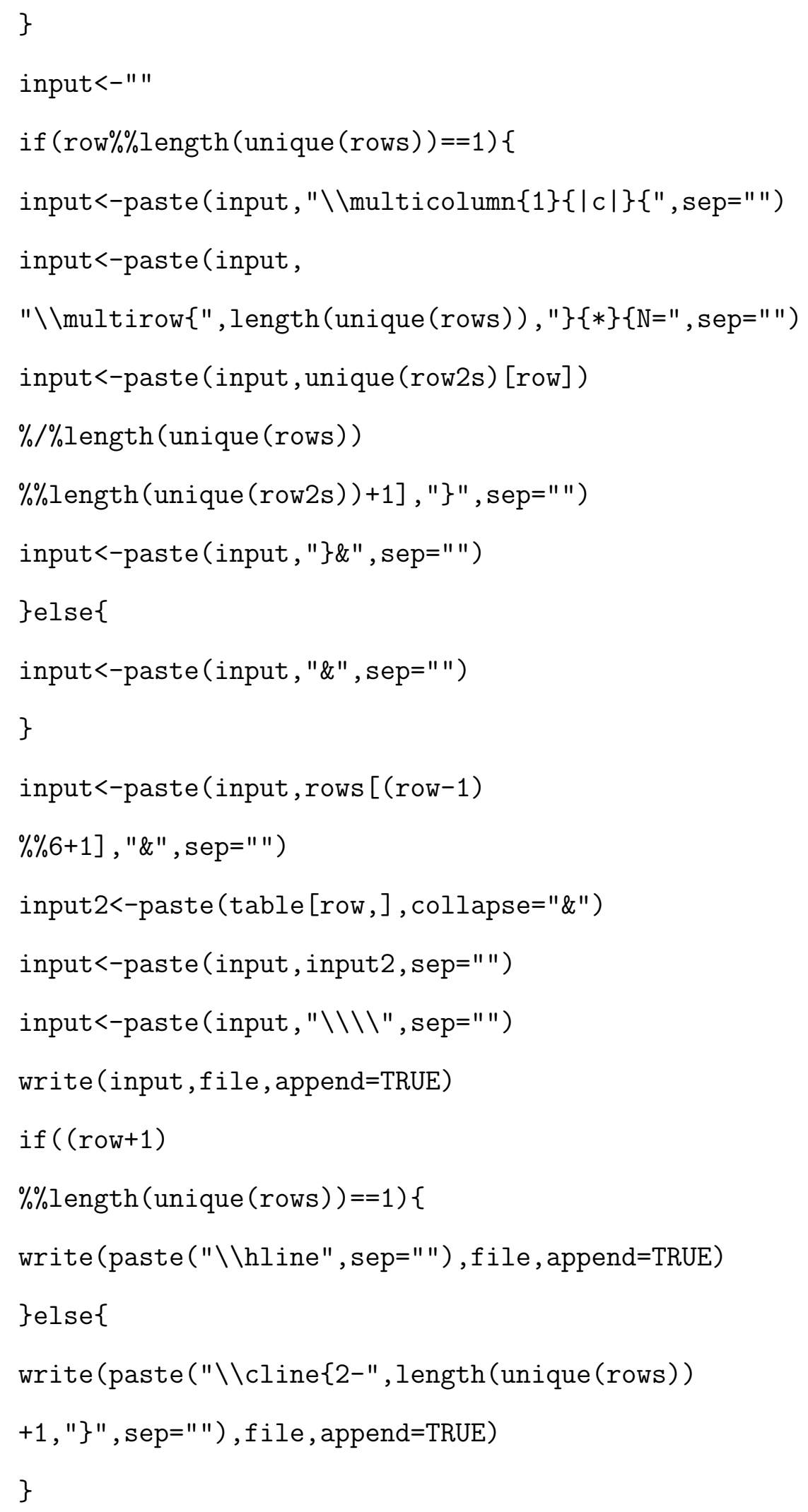




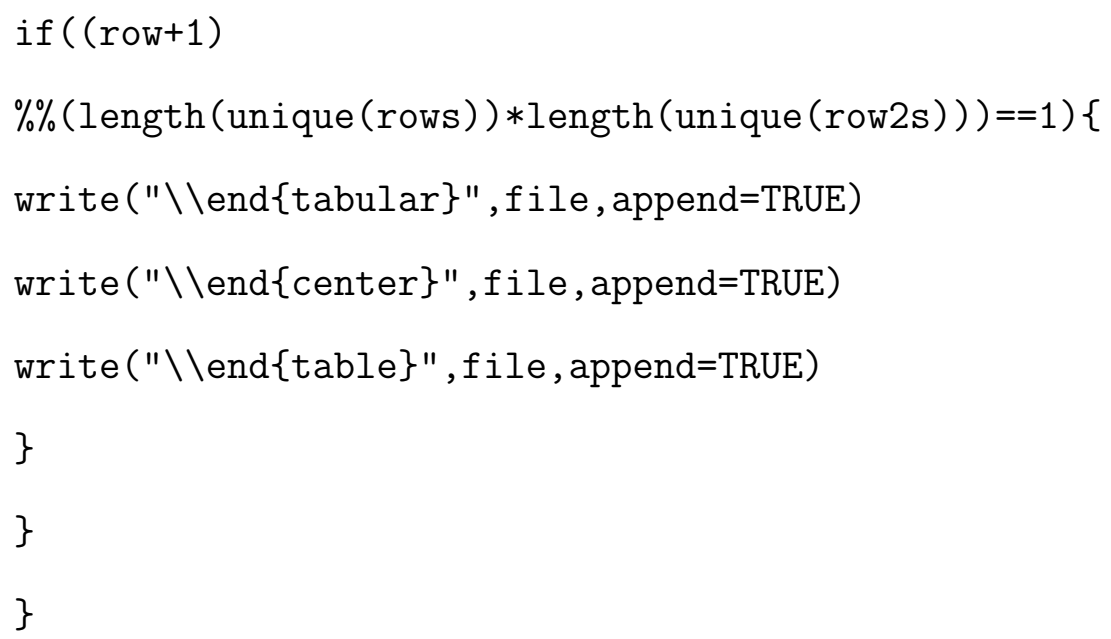

5. Solve for the MSE of the propensity score method

The simulation results did not return the propensity score method, so this code finds the needed values.

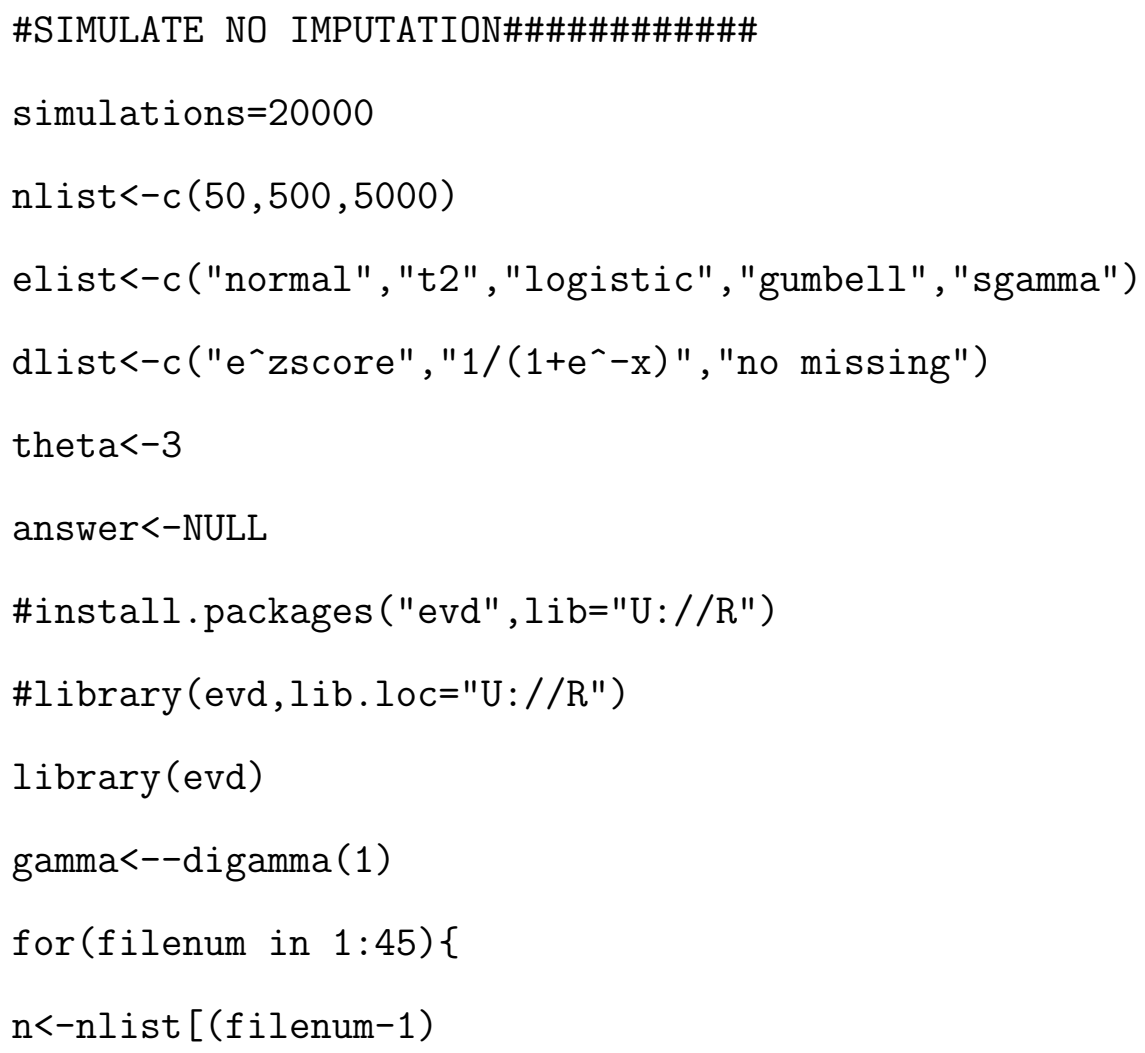




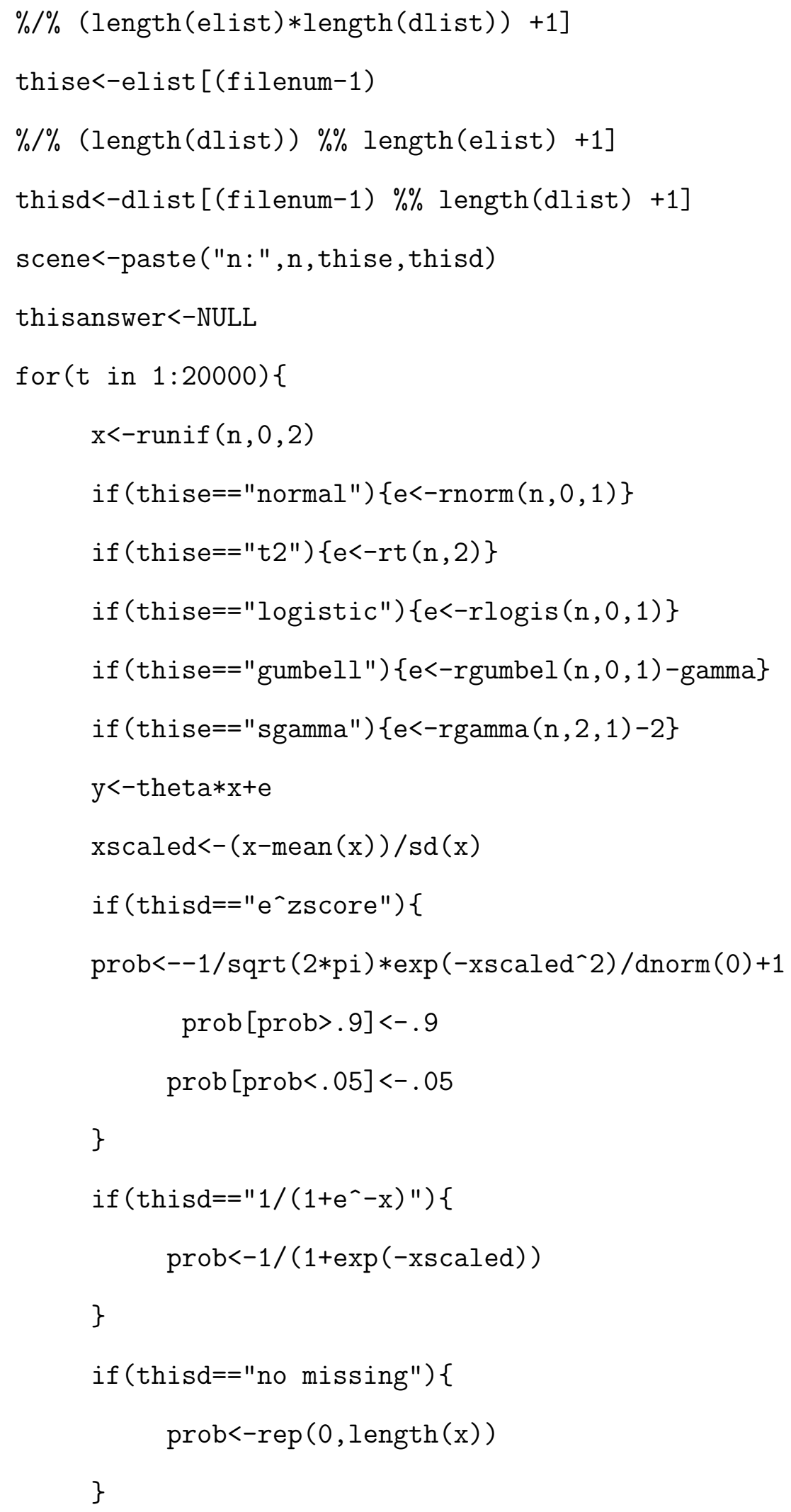




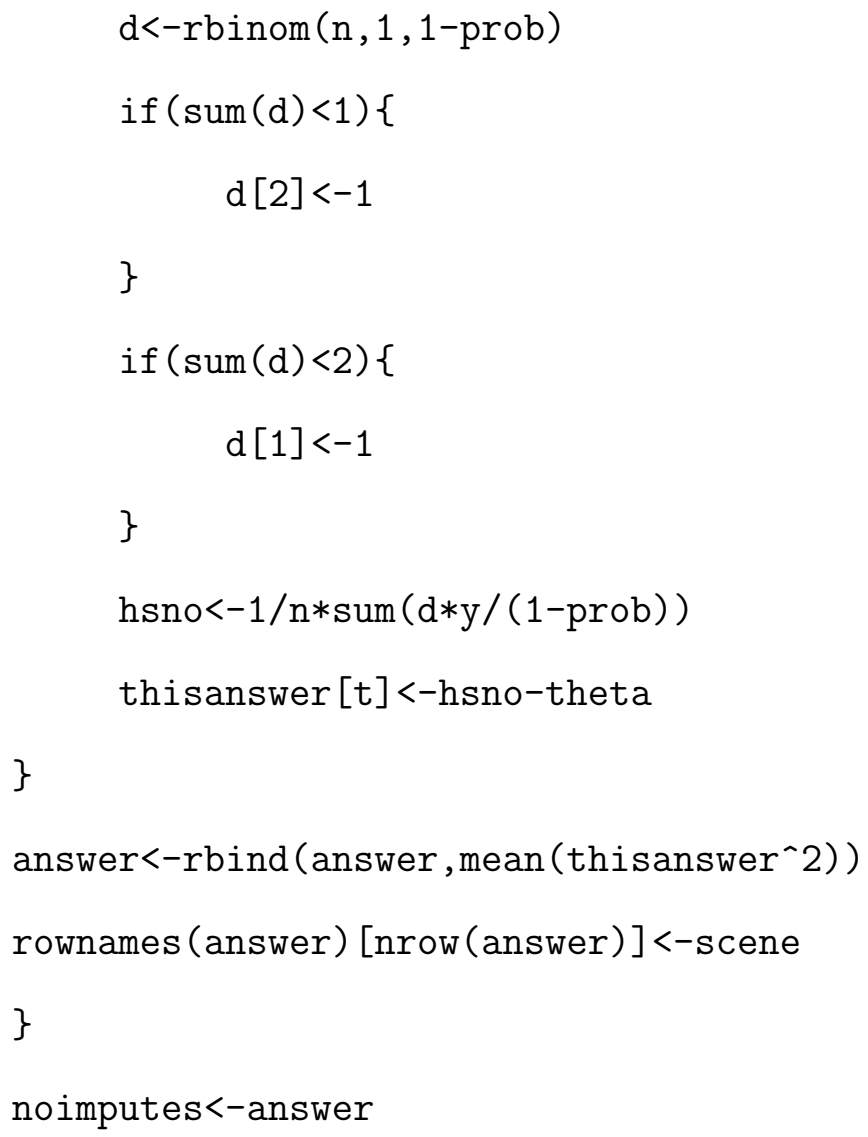

6. Graphs of the $\mathrm{E}(Y)$

The function "graphey" takes in a variable scene which determines the combination of error distribution and missingness structure. The variable pickn determines the sample size to graph.

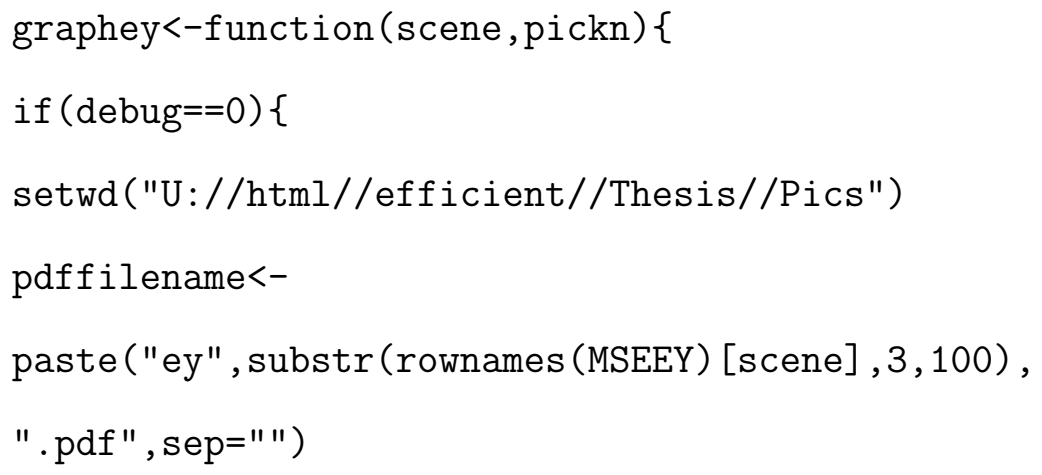




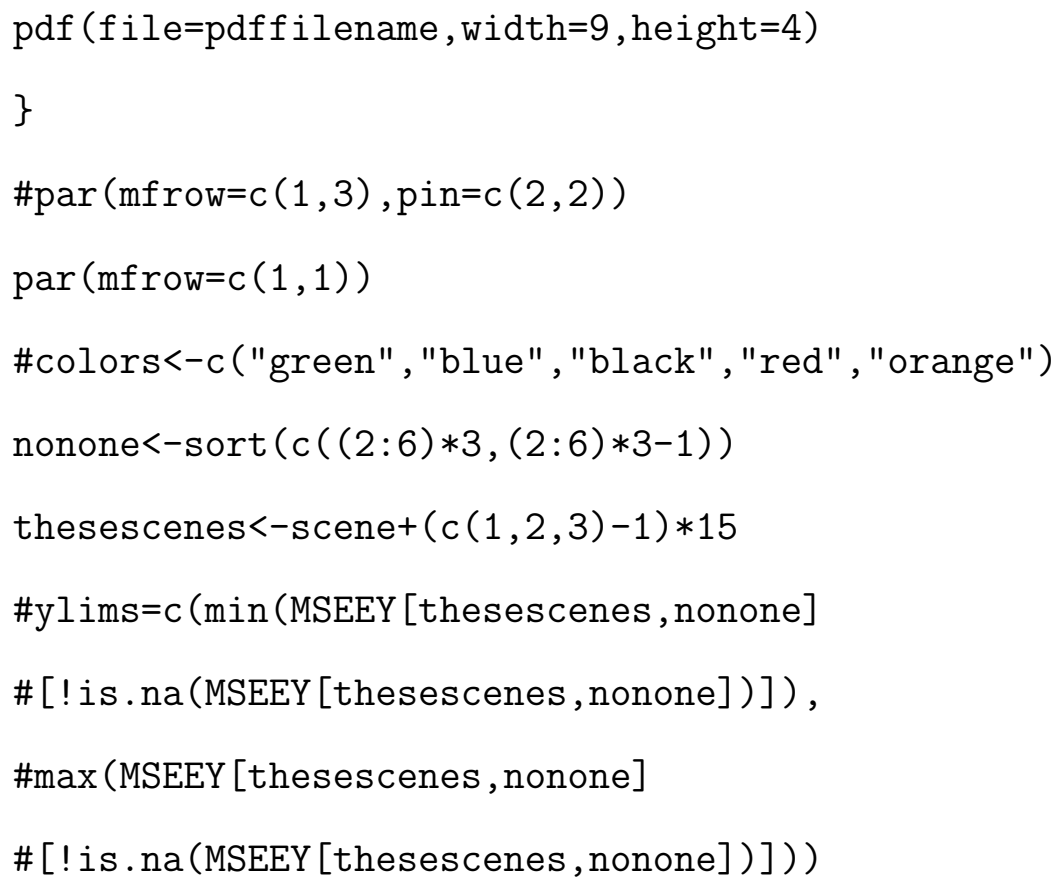




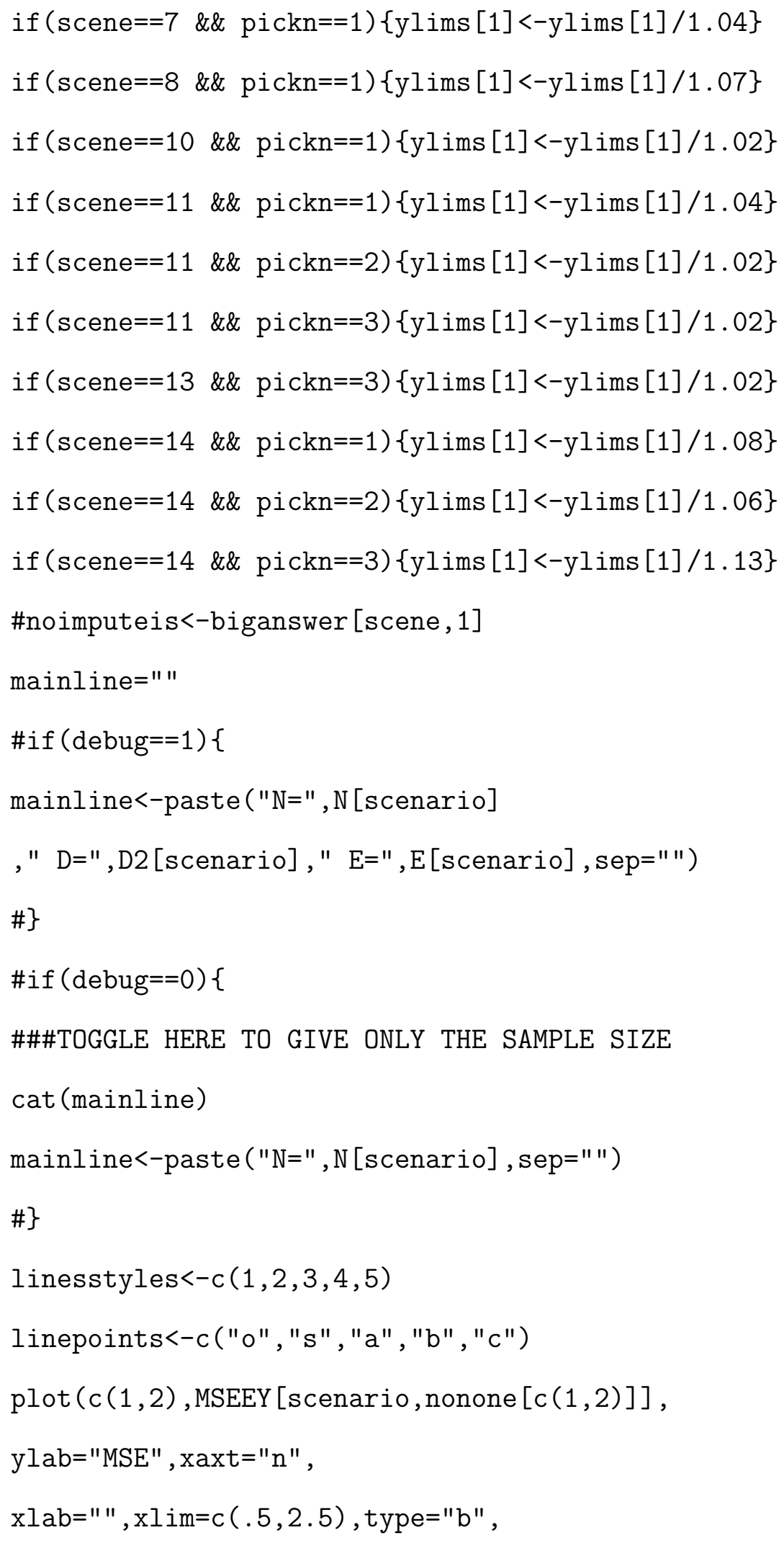




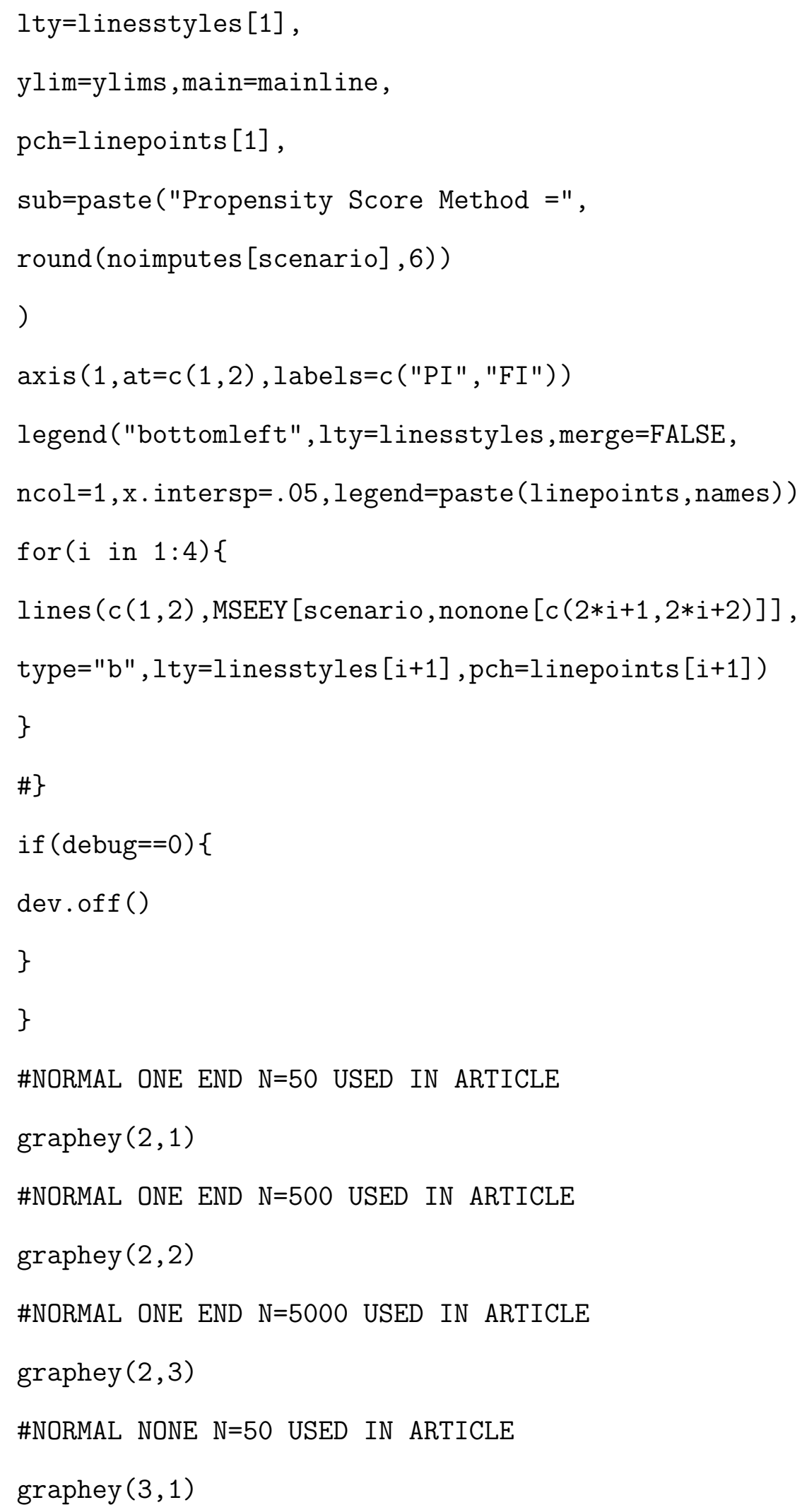


\#NORMAL NONE N=500 USED IN ARTICLE

graphey $(3,2)$

\#NORMAL NONE N=5000 USED IN ARTICLE

graphey $(3,3)$

\#GAMMA ONE END N=50 USED IN ARTICLE

graphey $(14,1)$

\#GAMMA ONE END N=500 USED IN ARTICLE

graphey $(14,2)$

\#GAMMA ONE END N=5000 USED IN ARTICLE

graphey $(14,3)$

7. Tables of MSE values for estimating $\mathrm{E}(\mathrm{Y})$

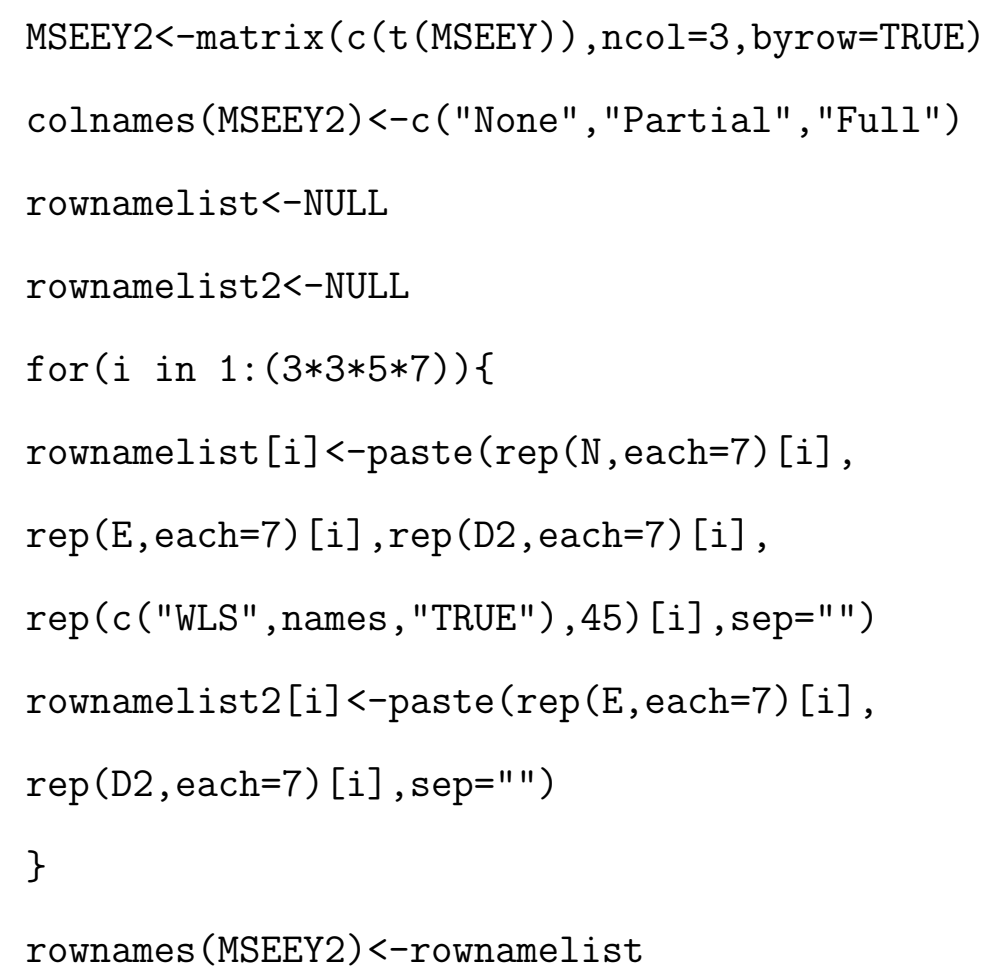




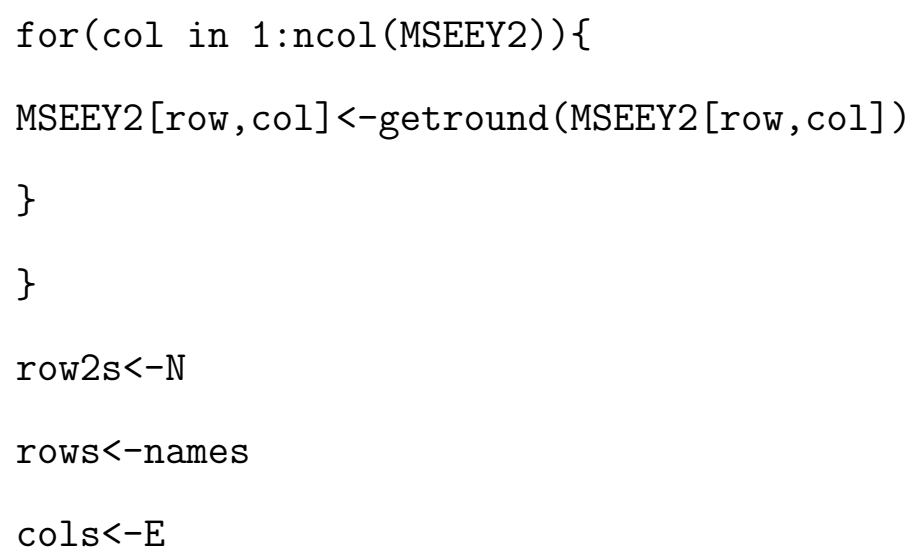


caps $2[4]<-"$ and the errors have a Gumbell distribution" caps $2[5]<-"$ and the errors have a Gamma distribution" caps $<-N U L L$ for (i in 1:15) \{caps [i]<-paste (rep (caps1, each=5) [i], $\operatorname{rep}(\operatorname{caps} 2,3)[i], \operatorname{sep}=" 1)\}$

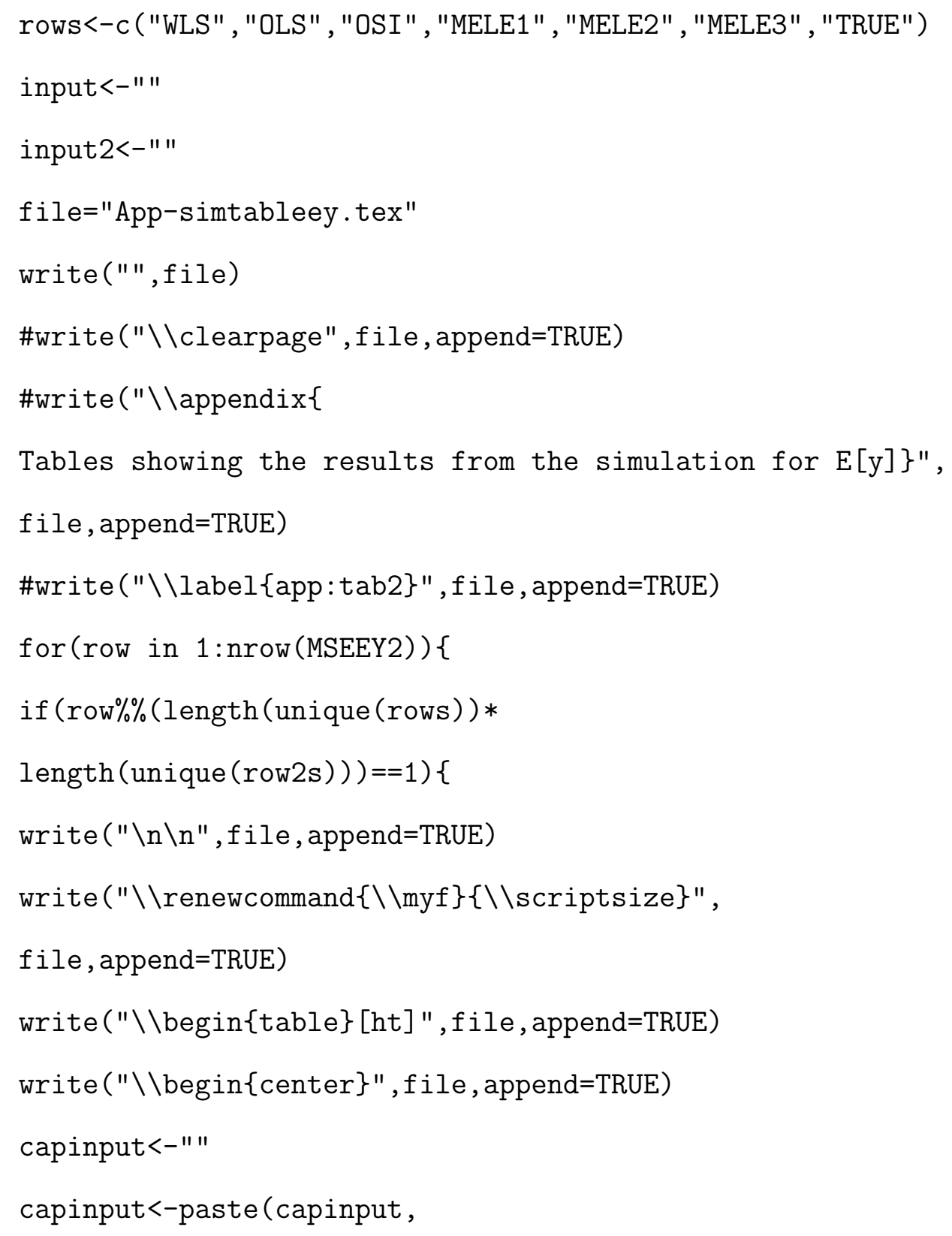




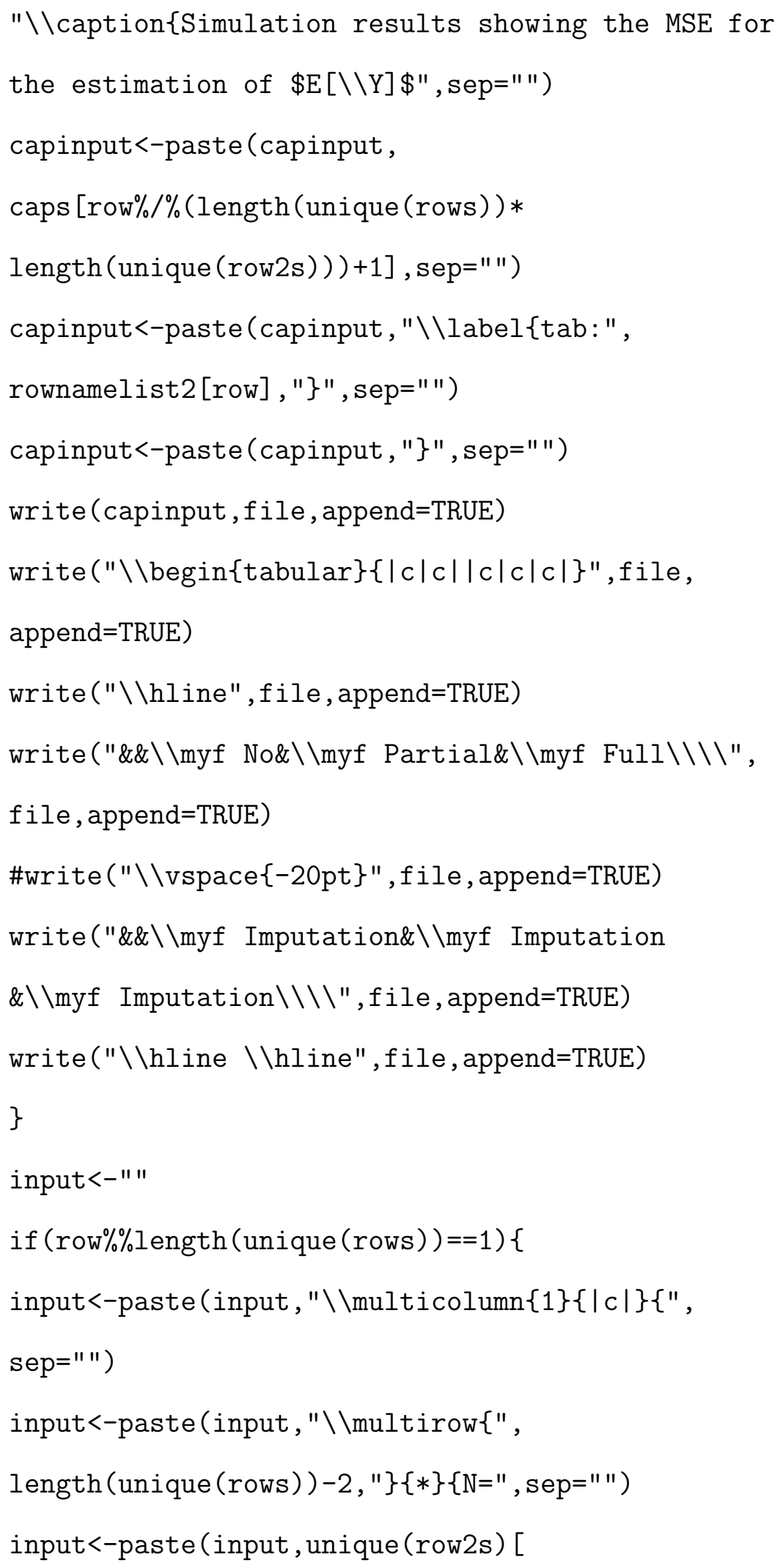




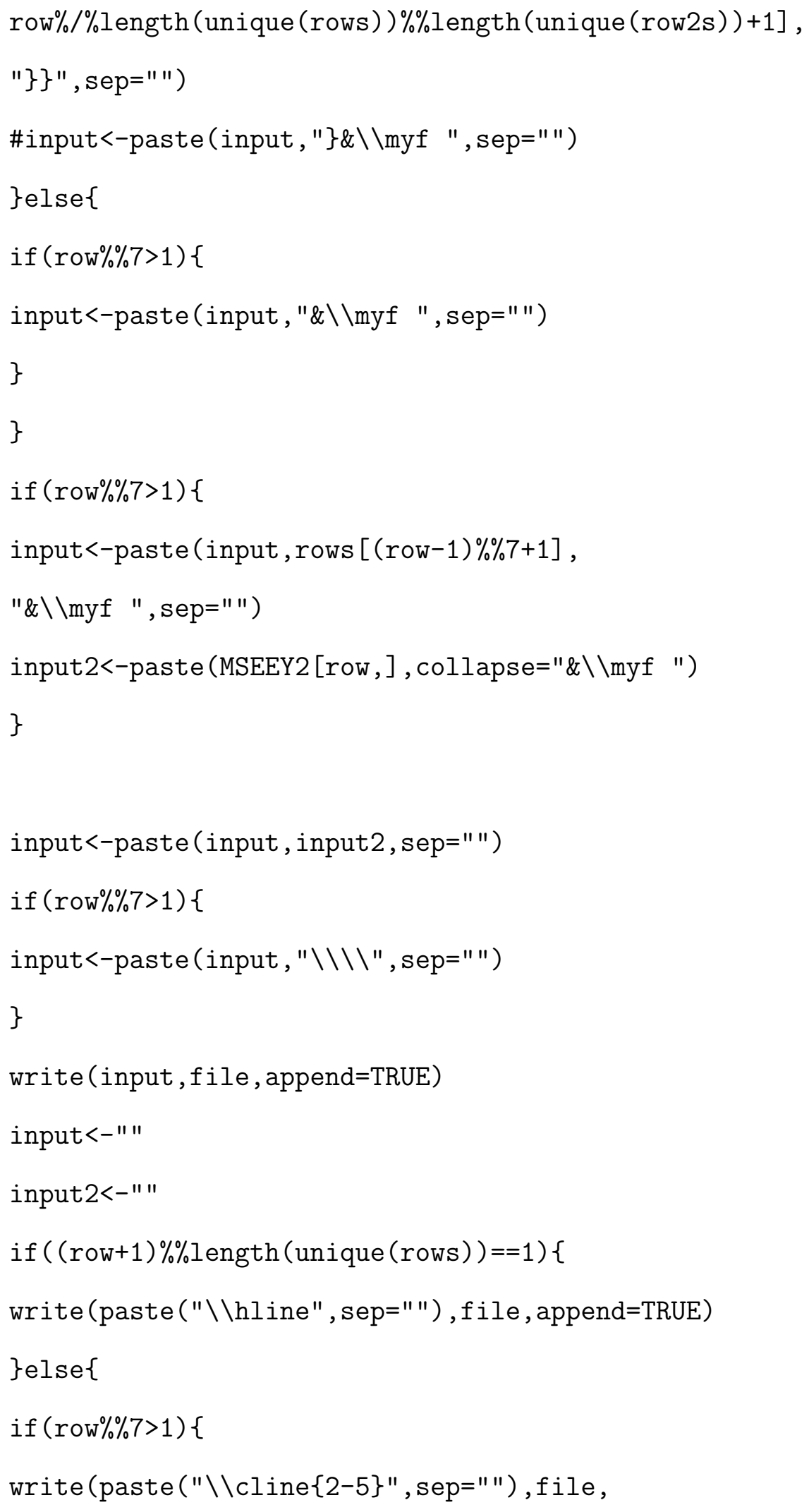




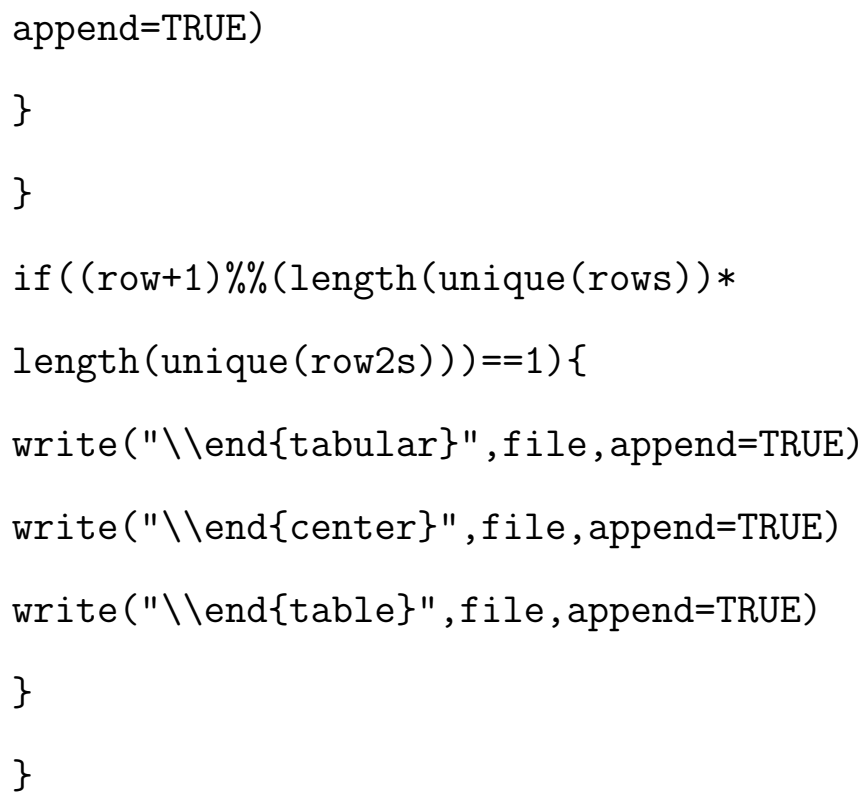

8. The asymptotic variances of estimators for $E(Y)$

The asymptotic variance for each example use different imputation methods as well as different estimates of $\vartheta$ and different error structures. The asymptotic variance for the weighted least squares estimates using the poor choice of weights is also calculated.

$t<-3$

$\mathrm{ex}<-1$

$\operatorname{exx}<-4 / 3$

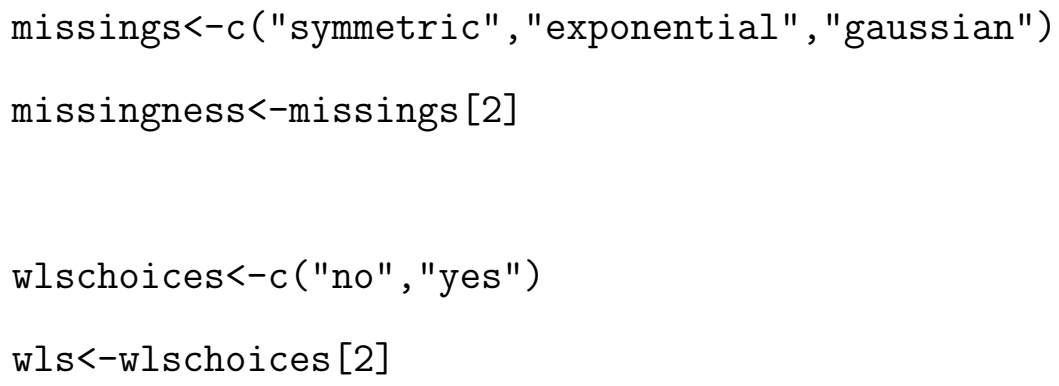




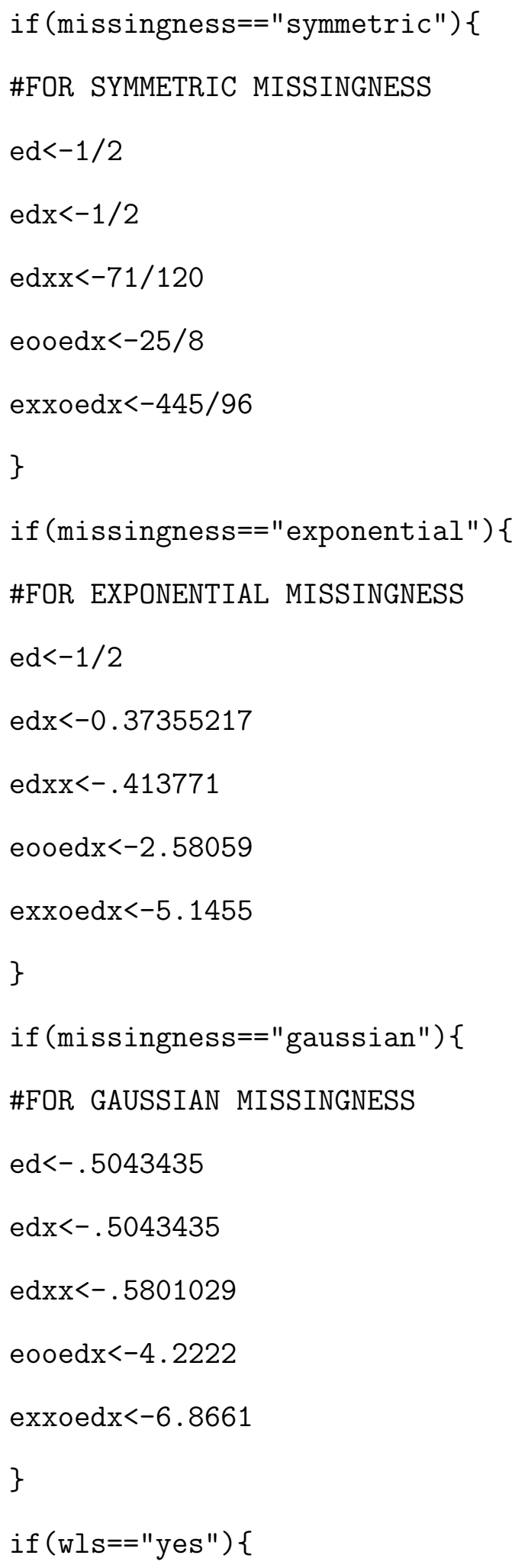




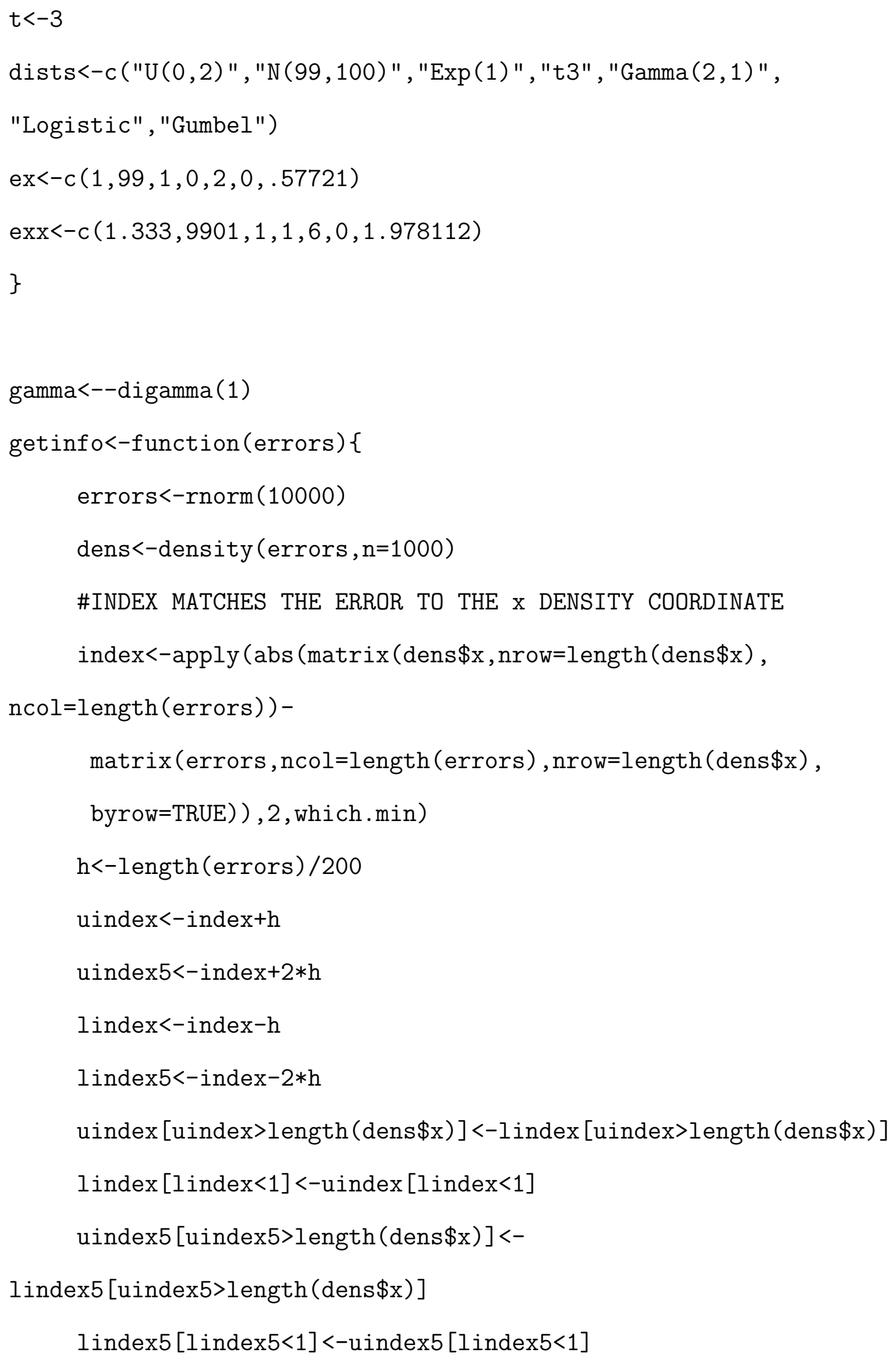




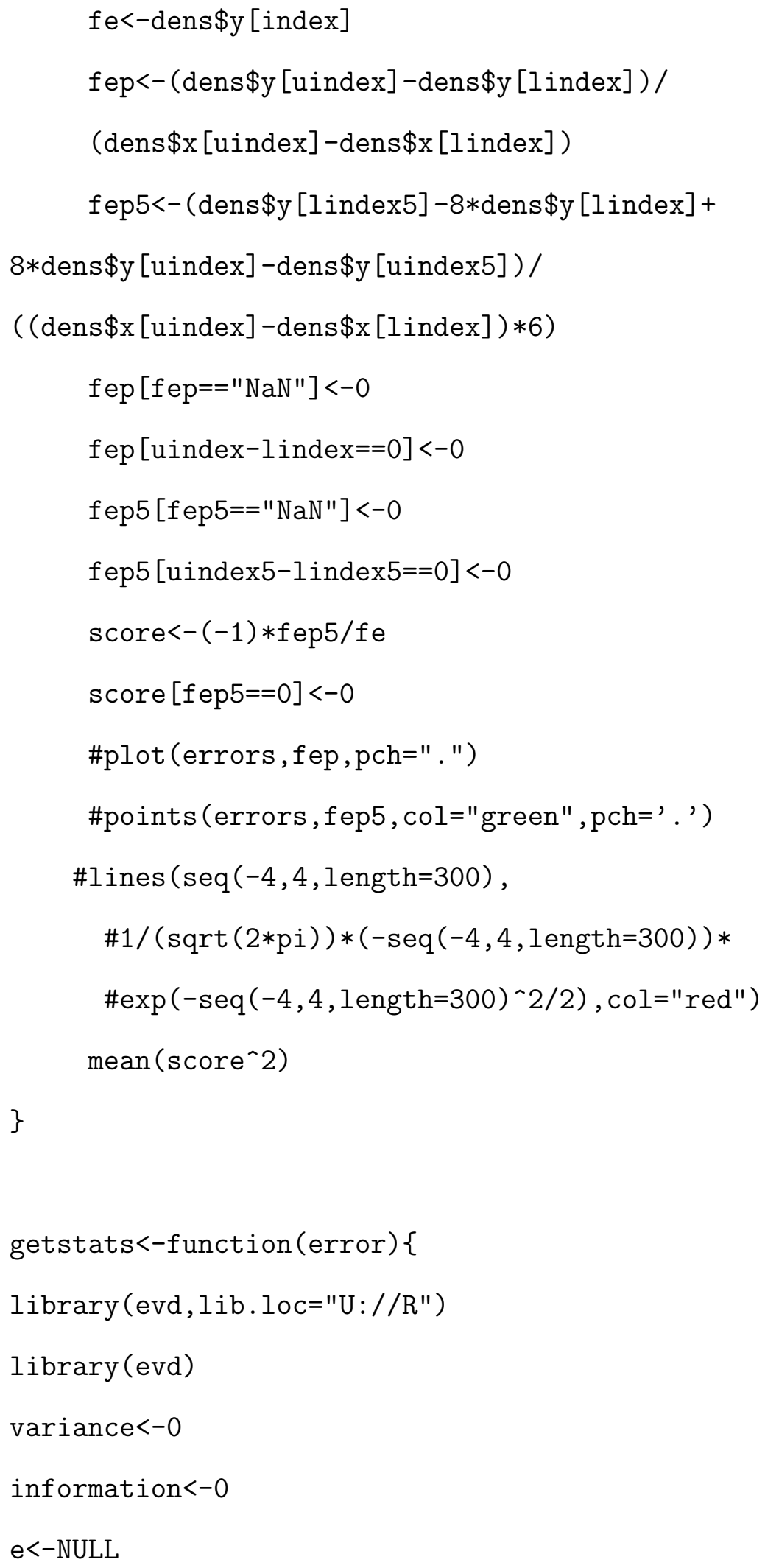




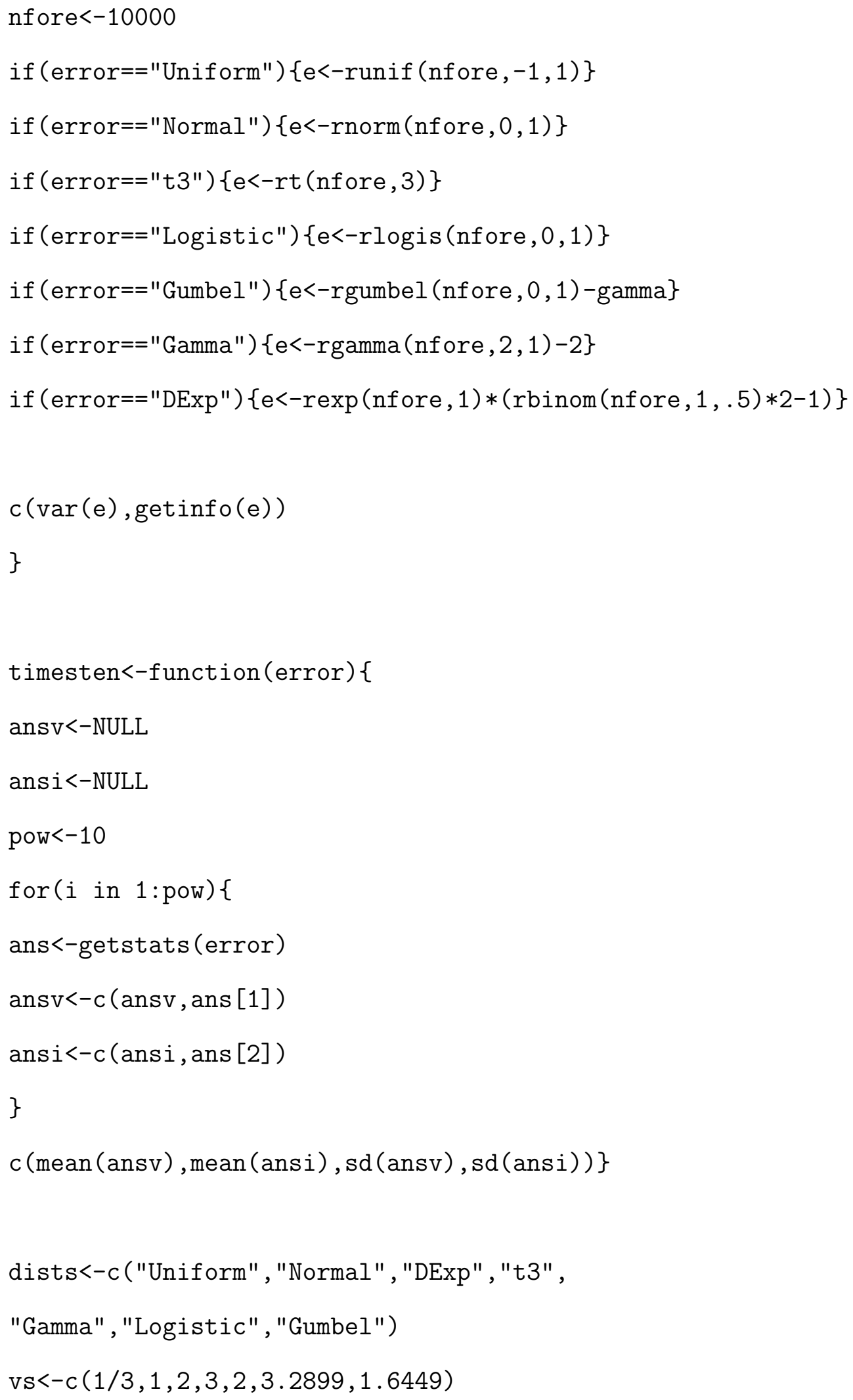


is $<-c(0,1,1,0.6667,1,0.3333,1)$

getcheck<-function( $)\{$

check<-as .data.frame (matrix $(0,8$, length (dists) ))

rownames (check)<-c("Theory v", "Sim v", "sd v", "Diff v",

"Theory I", "Sim I", "sd I", "Diff I")

colnames (check) $<$-dists

$\operatorname{check}[1]<$,- round (vs , 4)

check $[5]<$,- round (is , 4)

for ( $i$ in $1:$ length (dists)) \{

getcheck<-round (timesten (dists [i] ),4)

check $[2, i]<-\operatorname{getcheck}[1]$

check $[6, i]<-\operatorname{getcheck}[2]$

check $[3, i]<-\operatorname{get}$ check $[3]$

$\operatorname{check}[7, i]<-\operatorname{getcheck}[4]\}$

$\operatorname{check}[4]<$,- round (as numeric $(\operatorname{check}[1]$,$) -as numeric (\operatorname{check}[2]), 4$,

check $[8]<$,- round (as . numeric $(\operatorname{check}[5]$,$) -as . numeric (\operatorname{check}[6]), 4$,

check\}

edzz<-function $(v, i)\{e d x x * i-e d x * e d x / e d * i+e d x * e d x / e d * 1 / v\}$

edzzs<-edzz (vs, is)

roundit $<-3$

$\operatorname{avld}<-$ function $(v)\{$

ans $<-$ Inf

if $(e d x==e d * e x)\{$ 


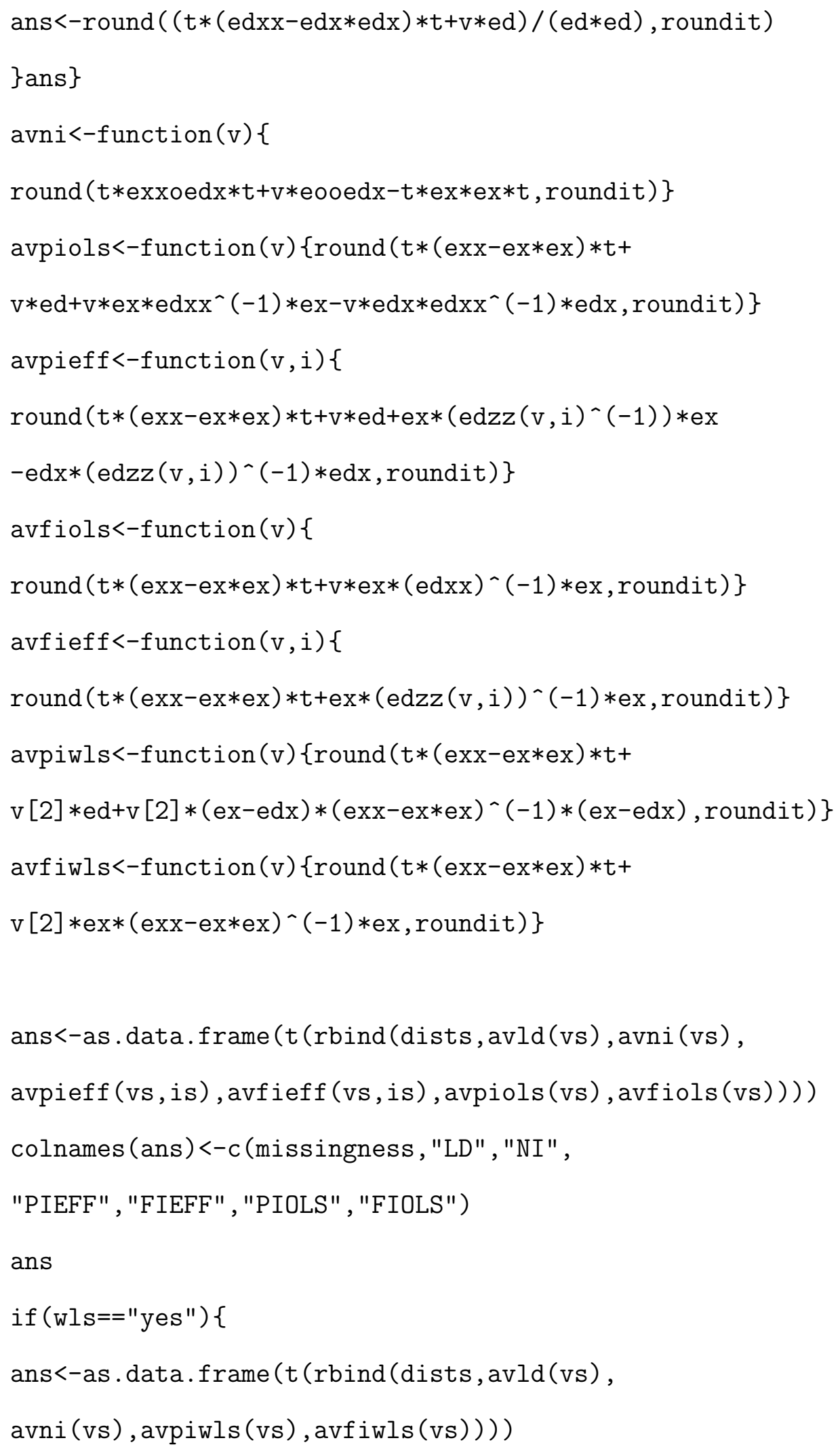




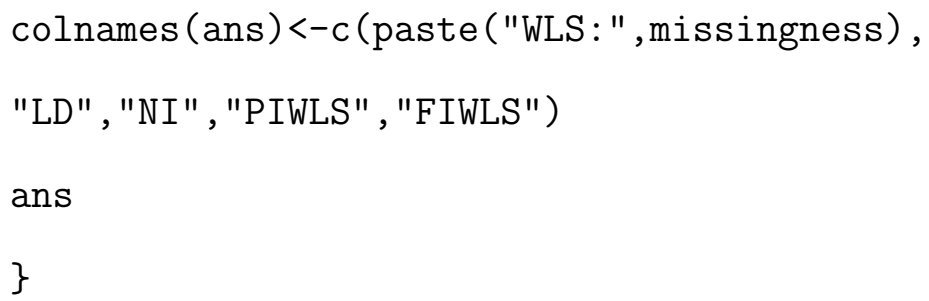




\section{Semi-parametric regression}

\section{Introduction}

In this section I introduce a much more complex model, and go through the formulation for the canonical gradient. By matching this canonical gradient to the influence function of an estimator it can be used to check if an estimator is efficient for this model. Such an efficient estimator has yet to be found, but the following derivation shows the incredible complexity that comes with a generalization of the model. The model is

$$
Y=r_{\vartheta}(X)+\gamma(Z)+\epsilon
$$

where $Y$ is the response, $r_{\vartheta}(X)$ is the known parametric function and $\vartheta$ is the unknown parameter for the random covariate $X$ with dimension $k_{1}, \gamma(Z)$ is the unknown non-parametric component for the random covariate $Z$ with dimension $k_{2}$, and $\epsilon$ is the random error term with distribution $f$ which has mean 0 and variance $\sigma^{2}$. See Müller et al. (2007) for details on estimating the error distribution of such a model. Further assume $X$ and $Z$ are independent of $\epsilon$.

The responses are MAR where

$$
\delta= \begin{cases}0 & \text { if } Y \text { is missing } \\ 1 & \text { if } Y \text { is not missing }\end{cases}
$$

the observed data is $(X, Z, \delta, \delta Y)$. See Wang et al. (2004) for details on the Missing At Random assumption for this model. The goal will be to estimate $E[Y]$. The joint 
distribution of the data is

$$
P(X, Z, Y, \delta)=G(d x, d z) B_{\pi(X, Z)}(d \delta)\left\{\delta Q(d y \mid X, Z)+(1-\delta) \delta_{o}(Y)\right\}
$$

where $G(d x, d z)$ is the marginal distribution of $(X, Z), B_{\pi(X, Z)}(d \delta)$ is the distribution of the conditional probability $\pi(X, Z)$ of $\delta=1, Q(d y \mid X, Z)$ is the conditional distribution of $Y$ given $(X=x, Z=z)$, and $\delta_{o}(Y)$ is the Dirac measure on $d y$ at 0 .

The canonical gradient is calculated by using Hellinger Derivatives to perturb the joint distribution and find the tangent space.

2. Perturbations through Hellinger derivatives

Lemma VI.1 The tangent space defined for the model above is

$$
\dot{P}_{n}=\{u(X, Z)+\delta v(Y, X, Z)+(\delta-\pi) w(X, Z)\} .
$$

where $u(X, Z), v(Y, X, Z)$, and $w(X, Z)$ are perturbations defined as the following Hellinger Derivatives

$$
\begin{aligned}
& G_{n u}(X, Z)=G(d x, d z)\left\{1+n^{-1 / 2} u(X, Z)\right\} \\
& Q_{n v}(Y \mid X, Z)=Q(d y \mid X, Z)\left\{1+n^{-1 / 2} v(Y, X, Z)\right\} \\
& B_{\pi n \pi(X, Z)}(d \delta)=B_{\pi(X, Z)}(d \delta)\left[1+n^{-1 / 2}\{\delta-\pi(X, Z)\} w(X, Z)\right]
\end{aligned}
$$

with the constraints that each perturbation is square integrable and

$$
\begin{aligned}
\int u(x, z) G(d x, d z) & =0 \\
\int v(y, x, z) Q(d y \mid x, z) & =0 .
\end{aligned}
$$


Proof: The use of $\sqrt{n}$ consistency in a semi-parametric model is explained in Schick (1996a). Use the notation given above for the model and the derivatives with each constraint. It may be also noted that

$$
Q(d y \mid X, Z)=f\left\{Y-r_{\vartheta}(X)-\gamma(Z)\right\} d y
$$

which will be incorporated later. The proof will show that

$$
P_{\text {nuwv }}(X, Z, Y, \delta)=P(X, Z, Y, \delta)\left(1+n^{-1 / 2} \dot{P}_{n}\right)
$$

The perturbed probability model is defined by

$$
\begin{aligned}
& P_{n u w v}(X, Z, Y, \delta) \\
= & G_{n u}(X, Z) B_{\pi n \pi(X, Z)}(d \delta)\left\{\delta Q_{n v}(Y \mid X, Z)+(1-\delta) \delta_{o}(Y)\right\} \\
= & G(d x, d z)\left\{1+n^{-1 / 2} u(X, Z)\right\} B_{\pi(X, Z)}(d \delta)\left[1+n^{-1 / 2}\{\delta-\pi(X, Z)\} w(X, Z)\right] \\
& {\left[\delta Q(d y \mid X, Z)\left\{1+n^{-1 / 2} v(Y, X, Z)\right\}+(1-\delta) \delta_{o}(Y)\right] . }
\end{aligned}
$$


This expands into

$$
\begin{aligned}
& P_{\text {nuwv }}(X, Z, Y, \delta) \\
= & G(d x, d z) B_{\pi(X, Z)}(d \delta) Q(d y \mid X, Z) \delta \\
& +G(d x, d z) B_{\pi(X, Z)}(d \delta) Q(d y \mid X, Z) \delta n^{-1 / 2} v(Y, X, Z) \\
& +G(d x, d z) B_{\pi(X, Z)}(d \delta)(1-\delta) \delta_{o}(Y) \\
& +G(d x, d z) B_{\pi(X, Z)}(d \delta) Q(d y \mid X, Z) n^{-1 / 2}\{\delta-\pi(X, Z)\} w(X, Z) \delta \\
& +G(d x, d z) B_{\pi(X, Z)}(d \delta) Q(d y \mid X, Z) n^{-1}\{\delta-\pi(X, Z)\} w(X, Z) \delta v(Y, X, Z) \\
& +G(d x, d z) B_{\pi(X, Z)}(d \delta) n^{-1 / 2}\{\delta-\pi(X, Z)\} w(X, Z)(1-\delta) \delta_{o}(Y) \\
& +G(d x, d z) B_{\pi(X, Z)}(d \delta) Q(d y \mid X, Z) n^{-1 / 2} u(X, Z) \delta \\
& +G(d x, d z) B_{\pi(X, Z)}(d \delta) Q(d y \mid X, Z) n^{-1} u(X, Z) \delta v(Y, X, Z) \\
& +G(d x, d z) B_{\pi(X, Z)}(d \delta) n^{1 / 2} u(X, Z)(1-\delta) \delta_{o}(Y) \\
& +G(d x, d z) B_{\pi(X, Z)}(d \delta) Q(d y \mid X, Z) n^{-1} u(X, Z)\{\delta-\pi(X, Z)\} w(X, Z) \delta \\
+ & G(d x, d z) B_{\pi(X, Z)}(d \delta) Q(d y \mid X, Z) n^{-3 / 2} u(X, Z)\{\delta-\pi(X, Z)\} w(X, Z) \delta v(Y, X, Z) \\
+ & G(d x, d z) B_{\pi(X, Z)}(d \delta) n^{-1} u(X, Z)\{\delta-\pi(X, Z)\} w(X, Z)(1-\delta) \delta_{o}(Y)
\end{aligned}
$$


Any term with $n$ to a power greater than $1 / 2$ can be put into $o_{p}\left(n^{-1 / 2}\right)$, so

$$
\begin{aligned}
& P_{\text {nuwv }}(X, Z, Y, \delta) \\
= & G(d x, d z) B_{\pi(X, Z)}(d \delta) Q(d y \mid X, Z) \delta \\
& +G(d x, d z) B_{\pi(X, Z)}(d \delta) Q(d y \mid X, Z) \delta n^{-1 / 2} v(Y, X, Z) \\
& +G(d x, d z) B_{\pi(X, Z)}(d \delta)(1-\delta) \delta_{o}(Y) \\
& +G(d x, d z) B_{\pi(X, Z)}(d \delta) Q(d y \mid X, Z) n^{-1 / 2}\{\delta-\pi(X, Z)\} w(X, Z) \delta \\
& +G(d x, d z) B_{\pi(X, Z)}(d \delta) n^{-1 / 2}\{\delta-\pi(X, Z)\} w(X, Z)(1-\delta) \delta_{o}(Y) \\
& +G(d x, d z) B_{\pi(X, Z)}(d \delta) Q(d y \mid X, Z) n^{-1 / 2} u(X, Z) \delta \\
& +G(d x, d z) B_{\pi(X, Z)}(d \delta) n^{1 / 2} u(X, Z)(1-\delta) \delta_{o}(Y) \\
& +o_{p}\left(n^{-1 / 2}\right) .
\end{aligned}
$$

After combining the like terms this becomes

$$
\begin{aligned}
& P_{\text {nuwv }}(X, Z, Y, \delta) \\
= & G(d x, d z) B_{\pi(X, Z)}(d \delta)\left\{\delta Q(d y \mid X, Z)+(1-\delta) \delta_{o}(Y)\right\} \\
& +G(d x, d z) B_{\pi(X, Z)}(d \delta) Q(d y \mid X, Z) n^{-1 / 2} \\
& {[\delta u(X, Z)+\delta v(Y, X, Z)+\{\delta-\pi(X, Z)\} w(X, Z) \delta] } \\
& +G(d x, d z) B_{\pi(X, Z)}(d \delta) n^{-1 / 2}(1-\delta) \delta_{o}(Y)[\{\delta-\pi(X, Z)\} w(X, Z)+u(X, Z)] \\
& +o_{p}\left(n^{-1 / 2}\right) .
\end{aligned}
$$

The first term is $P(X, Z, Y, \delta)$. Since $\delta(1-\delta)$ is 0 with probability 1 , a term is 
introduced at the end,

$$
\begin{aligned}
& P_{\text {nuwv }}(X, Z, Y, \delta) \\
= & P(X, Z, Y, \delta) \\
& +n^{-1 / 2} G(d x, d z) B_{\pi(X, Z)}(d \delta) \\
& (\delta Q(d y \mid X, Z)[u(X, Z)+\delta v(Y, X, Z)+\{\delta-\pi(X, Z)\} w(X, Z)]) \\
& +n^{-1 / 2} G(d x, d z) B_{\pi(X, Z)}(d \delta)\left((1-\delta) \delta_{o}(Y)[u(X, Z)+\right. \\
& \{\delta-\pi(X, Z)\} w(X, Z)]) \\
& +n^{-1 / 2} G(d x, d z) B_{\pi(X, Z)}(d \delta) \delta(1-\delta) \delta_{o}(Y) v(Y, X, Z) \\
= & P(X, Z, Y, \delta) \\
& +n^{-1 / 2} G(d x, d z) B_{\pi(X, Z)}(d \delta)\left\{\delta Q(d y \mid X, Z)+(1-\delta) \delta_{o}(Y)\right\} \\
& {[u(X, Z)+\delta v(Y, X, Z)+\{\delta-\pi(X, Z)\} w(X, Z)] } \\
& +o_{p}\left(n^{-1 / 2}\right) \\
& P(X, Z, Y, \delta)\left(1+n^{-1 / 2}[u(X, Z)+\delta v(Y, X, Z)+\{\delta-\pi(X, Z)\} w(X, Z)]\right) \\
+ & o_{p}\left(n^{-1 / 2}\right) . \\
&
\end{aligned}
$$

Therefore the tangent space of $P(X, Z, Y, \delta)$ is

$$
\dot{P}_{n}=\{u(X, Z)+\delta v(Y, X, Z)+\{\delta-\pi(X, Z)\} w(X, Z)\}
$$

where

$$
\begin{aligned}
& u(X, Z) \in L_{2,0}(G) \\
& v(Y, X, Z) \in L_{2,0}(Q) \\
& w(X, Z) \in L_{2,0}(B) .
\end{aligned}
$$




\section{Hajek-Le Cam convolution theorem}

An estimator $\hat{\chi}$ for $\chi(G, B, Q)$ is regular with limit $\mathcal{L}$ if $\mathcal{L}$ is a random variable such that under $P_{\text {nuwv }}(X, Z, Y, \delta)$

$$
n^{1 / 2}\{\hat{\chi}-\chi(G, B, Q)\} \rightarrow \mathcal{L}
$$

The convolution theorem says that $\mathcal{L}$ is distributed as the sum of two random variables, the first being normal with mean zero and variance $E\left[\left(g r_{(\vartheta, \gamma)}\right)^{2}\right]$, and the second being independent to the first. Thus $\hat{\chi}$ is efficient if it is regular with limit $N\left(0, E\left[\left(g r_{(\vartheta, \gamma)}\right)^{2}\right]\right)$. Using the definition of the influence function inf $\in L_{2,0}(P)$,

$$
n^{-1 / 2}\left\{\hat{\chi}-\chi\left(G_{n u}, Q_{n w}, Q_{n v}\right)\right\} \rightarrow n^{-1 / 2} \sum_{i=1}^{n} \inf +o_{p}(1) .
$$

This means a regular estimator is efficient if and only if it is asymptotically linear with influence function inf $=g r_{(\vartheta, \gamma)}$ where $g r_{(\vartheta, \gamma)}$ is the canonical gradient function. The canonical gradient is defined by

$$
n^{1 / 2}\left\{\hat{\chi}-\chi\left(G_{n u}, Q_{n w}, Q_{n v}\right)\right\} \rightarrow E\left\{g r_{(\vartheta, \gamma)}^{\top} g r_{\left(\vartheta_{*}, \gamma_{*}\right)}\right\}
$$

where $g r_{(\vartheta, \gamma)}$ is any gradient in the the tangent space, and $g r_{\left(\vartheta_{*}, \gamma_{*}\right)}$ is the canonical gradient. The canonical gradient is a gradient which is in the tangent space of the model. The tangent space is found by perturbing the unknown elements of the model. For the model under consideration the gradient will be an element of $\dot{P}_{n}$, so define the canonical gradient as

$$
g r_{\left(\vartheta_{*}, \gamma_{*}\right)}=u_{*}(X, Z)+\delta v_{*}(Y, X, Z)+\{\delta-\pi(X, Z)\} w_{*}(X, Z)
$$


The following lemma will simplify the characterization used by Equation A.4 for this canonical gradient.

Lemma VI.2 For the model defined above, the characterization for the canonical gradient is

$$
\begin{aligned}
& E\{Y u(X, Z)\}+E\{Y v(Y, X, Z)\} \\
= & E\left\{u_{*}(X, Z) u(X, Z)\right\}+E\left\{\delta v_{*}(Y, X, Z) v(Y, X, Z)\right\} \\
+ & E\left[\{\delta-\pi(X, Z)\}^{2} w_{*}(X, Z) w(X, Z)\right] .
\end{aligned}
$$

Proof: For this model,

$$
\begin{aligned}
& \chi\left(G_{n u}, Q_{n w}, Q_{n v}\right)= E_{n u w v}[Y]=\iiint Y G_{n u}(X, Z) B_{\pi n \pi(X, Z)}(d \delta) Q_{n v}(Y \mid X, Z) \\
& \chi(G, B, Q)=E[Y]=\iiint Y G(d x, d z) B_{\pi(X, Z)}(d \delta) Q(d y \mid X, Z) .
\end{aligned}
$$

This means the right hand side of Equation A.4 becomes

$$
\begin{aligned}
& \sqrt{n}\{\hat{\chi}-\chi(G, B, Q)\} \\
= & \sqrt{n}\left\{\iiint Y G_{n u}(X, Z) B_{\pi n \pi(X, Z)}(d \delta) Q_{n v}(Y \mid X, Z)\right. \\
& \left.-\iiint Y G(d x, d z) B_{\pi(X, Z)}(d \delta) Q(d y \mid X, Z)\right\} .
\end{aligned}
$$


Since $Y$ does not depend on $\delta, \pi(X, Z)$ or $w(X, Z)$,

$$
\begin{aligned}
& \sqrt{n}\{\hat{\chi}-\chi(G, B, Q)\} \\
= & \sqrt{n}\left\{\iint Y G_{n u}(X, Z) Q_{n v}(Y \mid X, Z)-\iint Y G(d x, d z) Q(d y \mid X, Z)\right\} \\
= & \sqrt{n}\left[\iint Y Q(d y \mid X, Z)\left\{1+n^{-1 / 2} v(Y, X, Z)\right\} G(d x, d z)\left\{1+n^{-1 / 2} u(X, Z)\right\}\right. \\
& \left.-\iint Y Q(d y \mid X, Z) G(d x, d z)\right] \\
= & \sqrt{n}\left[\int \int \left\{Y Q(d y \mid X, Z) G(d x, d z)+Y Q(d y \mid X, Z) n^{-1 / 2} v(Y, X, Z) G(d x, d z)\right.\right. \\
& +Y Q(d y \mid X, Z) G(d x, d z) n^{-1 / 2} u(X, Z)+ \\
& Y Q(d y \mid X, Z) G(d x, d z) n^{-1} u(X, Z) v(Y, X, Z) \\
& -Y Q(d y \mid X, Z) G(d x, d z)\}] .
\end{aligned}
$$

The term with $n^{-1}$ will approach zero, so

$$
\begin{aligned}
& \sqrt{n}\{\hat{\chi}-\chi(G, B, Q)\} \\
\rightarrow & \frac{\sqrt{n}}{\sqrt{n}} \iint[Y\{u(X, Z)+v(Y, X, Z)\} G(d x, d z) Q(d y \mid X, Z)] \\
= & E\{Y u(X, Z)\}+E\{Y v(Y, X, Z)\} .
\end{aligned}
$$

The right hand side of Equation A.4 can be simplified by Lemma VI.1.

$$
\begin{aligned}
E\left\{g r_{\left(\vartheta_{*}, \gamma_{*}\right)} g r_{(\vartheta, \gamma)}\right\}= & E\left(\left[u_{*}(X, Z)+\delta v_{*}(Y, X, Z)+\{\delta-\pi(X, Z)\} w_{*}(X, Z)\right]\right. \\
& {[u(X, Z)+\delta v(Y, X, Z)+\{\delta-\pi(X, Z)\} w(X, Z)]) }
\end{aligned}
$$

Since these perturbations were constrained to have a mean of zero, the cross product 
terms will be zero. Therefore

$$
\begin{aligned}
& E\left\{g r_{\left(\vartheta_{*}, \gamma_{*}\right)} g r_{(\vartheta, \gamma)}\right\} \\
= & E\left[u_{*}(X, Z) u(X, Z)+\delta v_{*}(Y, X, Z) v(Y, X, Z)+\right. \\
& \left.\{\delta-\pi(X, Z)\}^{2} w_{*}(X, Z) w(X, Z)\right] \\
= & E\left\{u_{*}(X, Z) u(X, Z)\right\}+E\left\{\delta v_{*}(Y, X, Z) v(Y, X, Z)\right\} \\
& +E\left[\{\delta-\pi(X, Z)\}^{2} w_{*}(X, Z) w(X, Z)\right] .
\end{aligned}
$$

Equating Equation A.6 to Equation A.7 we get

$$
\begin{aligned}
& E\{Y u(X, Z)\}+E\{Y v(Y, X, Z)\} \\
= & E\left\{u_{*}(X, Z) u(X, Z)\right\}+E\left\{\delta v_{*}(Y, X, Z) v(Y, X, Z)\right\} \\
+ & E\left[\{\delta-\pi(X, Z)\}^{2} w_{*}(X, Z) w(X, Z)\right] .
\end{aligned}
$$

Lemma VI.3 For the canonical gradient defined in Equation A.5, $w_{*}(X, Z)=0$, so the canonical gradient is

$$
g r_{\left(\vartheta_{*}, \gamma_{*}\right)}=u_{*}(X, Z)+\delta v_{*}(Y, X, Z) .
$$

Proof: The characterization given in Lemma VI.2 is true for any $u(X, Z)$ and $v(Y, X, Z)$ so set $u(X, Z)=0$ and $v(Y, X, Z)=0$. This yields

$$
0=E\left[\{\delta-\pi(X, Z)\}^{2} w_{*}(X, Z) w(X, Z)\right] .
$$

This equation must hold true for any $w(X, Z)$, so $w_{*}(X, Z)=0$. 
Lemma VI.4 For the canonical gradient defined in Lemma VI.3.

$$
u_{*}(X, Z)=r_{\vartheta}(X)+\gamma(Z)-E\left\{r_{\vartheta}(X)+\gamma(Z)\right\}
$$

This leaves the canonical gradient as

$$
g r_{\left(\vartheta_{*}, \gamma_{*}\right)}=r_{\vartheta}(X)+\gamma(Z)-E\left\{r_{\vartheta}(X)+\gamma(Z)\right\}+\delta v_{*}(Y, X, Z)
$$

Proof: The characterization given in Lemma VI.2 is true for any $v(Y, X, Z)$. From Lemma VI.3 we know $w_{*}(X, Z)=0$, so set $v(Y, X, Z)=0$. This leaves the characterization of the canonical gradient as

$$
E\{Y u(X, Z)\}=E\left\{u_{*}(X, Z) u(X, Z)\right\}
$$

The obvious solution to this equation is $u_{*}(X, Z)=Y$, but the restriction from Equation A.1 was that the expected value needs to be zero. The next obvious step is $Y-E(Y)$ but this no longer solves the equation. The solution that satisfies the equation and constraint is $E\{Y \mid(X, Z)\}-E(Y)$. This solves the equation because

$$
\begin{aligned}
E\left\{u_{*}(X, Z) u(X, Z)\right\} & =E([E\{Y \mid(X, Z)\}-E(Y)] u(X, Z)) \\
& =E[E\{Y \mid(X, Z)\} u(X, Z)]-E\{E(Y) u(X, Z)\} \\
& =E(E[E\{Y \mid(X, Z)\} u(X, Z) \mid(X, Z)])-E(Y) E\{u(X, Z)\} \\
& =E[E\{Y u(X, Z) \mid(X, Z)\}]-E(Y) E\{u(X, Z)\} \\
& =E\{Y u(X, Z)\} .
\end{aligned}
$$


The last step comes from $E\{u(X, Z)\}=0$. This satisfies Constraint A.1 because

$$
\begin{aligned}
\int u_{*}(X, Z) G(d x, d z) & =E[E\{Y \mid(X, Z)\}-E(Y)] \\
& =E[E\{Y \mid(X, Z)\}]-E(Y) \\
& =E(Y)-E(Y) \\
& =0 .
\end{aligned}
$$

This means

$$
\begin{aligned}
u_{*}(X, Z) & =E\{Y \mid(X, Z)\}-E(Y) \\
& =r_{\vartheta}(X)+\gamma(Z)-E\left\{r_{\vartheta}(X)+\gamma(Z)\right\} .
\end{aligned}
$$

The characterization for the canonical gradient given in Lemma VI.4 is true for any $u$ so setting $u=0$ the result is

$$
E\{Y v(Y, X, Z)\}=E\left\{\delta v_{*}(Y, X, Z) v(Y, X, Z)\right\} .
$$

This equation is not easily solved. This problem can be broken into smaller pieces by incorporating the restriction in Equation A.3

$$
Q(d y \mid X, Z)=f\left\{Y-r_{\vartheta}(X)-\gamma(Z)\right\} d y
$$

and defining further perturbations. Define the perturbations using the following Hellinger Derivatives,

$$
\begin{aligned}
f_{n s}(\epsilon) & =f(\epsilon)\left\{1+n^{-1 / 2} s(\epsilon)\right\} \\
\vartheta_{n t} & =\vartheta+n^{-1 / 2} t \\
\gamma_{n g}(Z) & =\gamma(Z)+n^{-1 / 2} g(Z) .
\end{aligned}
$$


for $t \in \mathbb{R}^{k_{1}}, g(Z)$ maps $\mathbb{R}^{k_{2}} \rightarrow \mathbb{R}, s(\epsilon) \in \mathbb{R}$ and subject to the constraints

$$
\begin{aligned}
& \int s(\epsilon) f(\epsilon) d \epsilon=0 \\
& \int \epsilon s(\epsilon) f(\epsilon) d \epsilon=0 .
\end{aligned}
$$

Which guarantees the needed assumptions for a valid error distribution, namely

$$
\begin{aligned}
E\left\{f_{n s}(\epsilon)\right\} & =0 \\
\int f_{n s}(\epsilon) d \epsilon & =1
\end{aligned}
$$

Lemma VI.5 Using the notation defined above,

$$
v_{*}(Y, X, Z)=s(\epsilon)+l(\epsilon)\left\{t^{\top} X+g(Z)\right\}
$$

PROOF: The perturbed $f$ is

$$
\begin{aligned}
f_{n s}\left(\epsilon_{t g}\right)= & f_{n s}\left\{Y-r_{\vartheta_{n t}}(X)-\gamma_{n g}(Z)\right\} \\
= & f\left\{Y-r_{\vartheta_{n t}}(X)-\gamma_{n g}(Z)\right\} \\
& {\left[1+n^{-1 / 2} s\left\{Y-r_{\vartheta_{n t}}(X)-\gamma_{n g}(Z)\right\}\right] } \\
= & f\left[Y-\left\{r_{\vartheta_{n t}}(X)+\gamma(Z)+n^{-1 / 2} g(Z)\right\}\right] \\
& \left(1+n^{-1 / 2} s\left[Y-\left\{r_{\vartheta_{n t}}(X)+\gamma(Z)+n^{-1 / 2} g(Z)\right\}\right]\right) .
\end{aligned}
$$

To simplify note that by using a Taylor Series expansion where $\dot{r}_{\vartheta}(X)$ is the derivative 
of $r_{\vartheta}(X)$,

$$
\begin{aligned}
r_{\vartheta_{n t}}(X) & =r_{\vartheta}(X)+\left(\vartheta_{n t}-\vartheta\right)^{\top} \dot{r}_{\vartheta}(X)+o_{p}\left(n^{-1 / 2}\right) \\
& =r_{\vartheta}(X)+\left(\vartheta+n^{-1 / 2} t-\vartheta\right)^{\top} \dot{r}_{\vartheta}(X)+o_{p}\left(n^{-1 / 2}\right) \\
& =r_{\vartheta}(X)+n^{-1 / 2} t^{\top} \dot{r}_{\vartheta}(X)+o_{p}\left(n^{-1 / 2}\right) .
\end{aligned}
$$

By substituting this into Equation A.12

$$
\begin{aligned}
f_{n s}\left(\epsilon_{t g}\right)= & f\left[Y-\left\{r_{\vartheta}(X)+n^{-1 / 2} t^{\top} \dot{r}_{\vartheta}(X)+\gamma(Z)+n^{-1 / 2} g(Z)\right\}\right] \\
& \left(1+n^{-1 / 2} s\left[Y-\left\{r_{\vartheta}(X)+n^{-1 / 2} t^{\top} \dot{r}_{\vartheta}(X)+\gamma(Z)+n^{-1 / 2} g(Z)\right\}\right]\right) \\
= & f\left[Y-\left\{r_{\vartheta}(X)+\gamma(Z)+n^{-1 / 2} t^{\top} \dot{r}_{\vartheta}(X)+n^{-1 / 2} g(Z)\right\}\right] \\
& \left(1+n^{-1 / 2} s\left[Y-\left\{r_{\vartheta}(X)+\gamma(Z)+n^{-1 / 2} t^{\top} \dot{r}_{\vartheta}(X)+n^{-1 / 2} g(Z)\right\}\right]\right) .
\end{aligned}
$$

To find the relationship between $f_{n s}\left(\epsilon_{t g}\right)$ and $f(\epsilon)$ I will use a Taylor Series expansion. To help simplify the calculation let $\Delta=-n^{-1 / 2}\left\{t^{\top} \dot{r}_{\vartheta}(X)+g(Z)\right\}$. Then equation becomes

$$
f_{n s}\left(\epsilon_{t g}\right)=f(\epsilon+\Delta)\left\{1+n^{-1 / 2} s(\epsilon+\Delta)\right\}
$$

Define $\dot{f}(\epsilon)$ as the derivative of $f(\epsilon)$ and $\dot{s}(\epsilon)$ as the derivative of $s(\epsilon)$. Then by Taylor Expansion

$$
\begin{aligned}
& f(\epsilon+\Delta)=f(\epsilon)+(\epsilon+\Delta-\epsilon) \dot{f}(\epsilon)+o_{p}\left(n^{-1 / 2}\right) \\
& s(\epsilon+\Delta)=s(\epsilon)+(\epsilon+\Delta-\epsilon) \dot{s}(\epsilon)+o_{p}\left(n^{-1 / 2}\right) .
\end{aligned}
$$


Using these Taylor Expansions with Equation A.13 yields

$$
\begin{aligned}
f_{n s}\left(\epsilon_{t g}\right)= & \{f(\epsilon)+\Delta \dot{f}(\epsilon)\}\left[1+n^{-1 / 2}\{s(\epsilon)+\Delta \dot{s}(\epsilon)\}\right]+o_{p}\left(n^{-1 / 2}\right) \\
= & {\left[f(\epsilon)-n^{-1 / 2}\left\{t^{\top} \dot{r}_{\vartheta}(X)+g(Z)\right\} \dot{f}(\epsilon)\right] } \\
& \left(1+n^{-1 / 2}\left[s(\epsilon)-n^{-1 / 2}\left\{t^{\top} \dot{r}_{\vartheta}(X)+g(Z)\right\} \dot{s}(\epsilon)\right]\right)+o_{p}\left(n^{-1 / 2}\right) \\
= & f(\epsilon)+f(\epsilon) n^{-1 / 2} s(\epsilon)-n^{-1} f(\epsilon) \dot{s}(\epsilon)\left\{t^{\top} \dot{r}_{\vartheta}(X)+g(Z)\right\} \\
& -n^{-1 / 2} \dot{f}(\epsilon)\left\{t^{\top} \dot{r}_{\vartheta}(X)+g(Z)\right\}-\dot{f}(\epsilon) s(\epsilon) n^{-1}\left\{t^{\top} \dot{r}_{\vartheta}(X)+g(Z)\right\} \\
& +n^{-3 / 2} \dot{f}(\epsilon) \dot{s}(\epsilon)\left\{t^{\top} \dot{r}_{\vartheta}(X)+g(Z)\right\}+o_{p}\left(n^{-1 / 2}\right) .
\end{aligned}
$$

Note that any term with $n$ raised to a negative power greater than $1 / 2$ can be put into the term $o_{p}\left(n^{-1 / 2}\right)$. Then

$$
\begin{aligned}
f_{n s}\left(\epsilon_{t g}\right) & =f(\epsilon)+f(\epsilon) n^{-1 / 2} s(\epsilon)-n^{-1 / 2} \dot{f}(\epsilon)\left\{t^{\top} \dot{r}_{\vartheta}(X)+g(Z)\right\} \\
& =f(\epsilon)\left(1+n^{-1 / 2}\left[s(\epsilon)-\frac{\dot{f}(\epsilon)}{f(\epsilon)}\left\{t^{\top} \dot{r}_{\vartheta}(X)+g(Z)\right\}\right]\right) .
\end{aligned}
$$

Define the score function $l(\epsilon)=-\frac{\dot{f}(\epsilon)}{f(\epsilon)}$, then

$$
f_{n s}\left(\epsilon_{t g}\right)=f(\epsilon)\left(1+n^{-1 / 2}\left[s(\epsilon)+l(\epsilon)\left\{t^{\top} \dot{r}_{\vartheta}(X)+g(Z)\right\}\right]\right)+o_{p}\left(n^{-1 / 2}\right)
$$

By comparing this result with Equation A.2 and Equation A.3 and noting that this perturbation is a function of $(X, Z, Y)$ it is clear

$$
v_{*}(Y, X, Z)=s(\epsilon)+l(\epsilon)\left\{t^{\top} \dot{r}_{\vartheta}(X)+g(Z)\right\}
$$

\section{Simplifying the tangent space}

Future calculations will be simplified by breaking $v_{*}(Y, X, Z)$ into two convenient pieces. To do this the following three lemmas will be helpful. The first shows that 
any function of $X$ and $\delta$ times $\epsilon$ is zero, the second shows $E\{\epsilon l(\epsilon)\}=1$, and the third how to pull $\delta$ out of an expected value.

Lemma VI.6 For the model as defined above for any function $g(X, \delta)$,

$$
E[g(X, \delta) \epsilon]=0
$$

\section{ProOF:}

$$
\begin{aligned}
E[g(X, \delta) \epsilon] & =E\left[g(X, \delta)\left(Y-\vartheta^{\top} X\right)\right] \\
& =E[g(X, \delta) Y]-E\left[g(X, \delta) \vartheta^{\top} X\right] \\
& =E[E\{g(X, \delta) Y \mid X\}]-E\left[E\left\{g(X, \delta) \vartheta^{\top} X \mid X\right\}\right] \\
& =E[E\{g(X, \delta) \mid X\} E\{Y \mid X\}]-E\left[E\{g(X, \delta) \mid X\} \vartheta^{\top} X\right] \\
& =E\left[E\{g(X, \delta) \mid X\} \vartheta^{\top} X\right]-E\left[E\{g(X, \delta) \mid X\} \vartheta^{\top} X\right] \\
& =0 .
\end{aligned}
$$

Lemma VI.7 For any random variable $\epsilon$ with distribution $f(\epsilon)$, finite expected value, and score function $l(\epsilon)$,

$$
E\{\epsilon l(\epsilon)\}=1
$$




\section{ProOF:}

$$
\begin{aligned}
E\{\epsilon l(\epsilon)\} & =\int \epsilon l(\epsilon) f(\epsilon) d \epsilon \\
& =-\int \epsilon \frac{\dot{f}(\epsilon)}{f(\epsilon)} f(\epsilon) d \epsilon \\
& =-\int \epsilon \dot{f}(\epsilon) d \epsilon .
\end{aligned}
$$

Then using integration by parts

$$
\begin{aligned}
E\{\epsilon l(\epsilon)\} & =-\{\epsilon f(\epsilon)\}_{-\infty}^{\infty}+\int f(\epsilon) d \epsilon \\
& =-\{\epsilon f(\epsilon)\}_{-\infty}^{\infty}+1 \\
& =\lim _{\epsilon \rightarrow-\infty} \epsilon f(\epsilon)-\lim _{\epsilon \rightarrow \infty} \epsilon f(\epsilon)+1
\end{aligned}
$$

Since the expected value of $\epsilon$ is finite,

$$
\int \epsilon f(\epsilon) d \epsilon=\int_{M}^{\infty} \epsilon f(\epsilon) d \epsilon+\int_{\infty}^{M} \epsilon f(\epsilon) d \epsilon<\infty
$$

by the Divergence Theorem the limit of $\epsilon f(\epsilon)$ as $\epsilon$ goes to $-\infty$ or $\infty$ will be zero. Thus

$$
E\{\epsilon l(\epsilon)\}=1 \text {. }
$$

Lemma VI.8 For any function $g(X, Z, \delta, Y)$

$$
E\{\delta g(X, Z, \delta, Y)\}=E(\delta) E\{g(X, Z, \delta, Y) \mid \delta=1\}
$$


Proof: The proof is trivial since $\delta$ can only take two values, 0 or 1 .

$$
\begin{aligned}
& E\{\delta g(X, Z, \delta, Y)\} \\
= & E\{1 g(X, Z, \delta, Y) \mid \delta=1\} P(\delta=1)+E\{0 g(X, Z, \delta, Y)\} P(\delta=1) \\
= & E\{g(X, Z, \delta, Y) \mid \delta=1\} E(\delta)
\end{aligned}
$$

With these two lemmas it will be easier to show the simplification of $v_{*}(Y, X, Z)$.

Lemma VI.9 Using the notation above $v_{*}(Y, X, Z)$ can be rewritten as the sum of the following pieces

$$
s_{2}(\epsilon)+\xi(X, Z, \epsilon)
$$

where

$$
\begin{aligned}
s_{2}(\epsilon) & \in \mathcal{S}=\{s(\epsilon)\} \\
\xi(X, Z, \epsilon) & =[\phi(X, Z)-E\{\phi(X, Z) \mid \delta=1\}] l(\epsilon)+E\{\phi(X, Z) \mid \delta=1\} \frac{\epsilon}{\sigma^{2}} \\
\phi(X, Z) & =t^{\top} \dot{r}_{\vartheta}(X)+g(Z)
\end{aligned}
$$

This is desirable because $s_{2} \epsilon$ is orthogonal to $\delta \xi(X, Z, \epsilon)$.

Proof: Using the definitions of $\xi(X, Z, \epsilon)$ and $\phi(X, Z)$ given in the statement of the lemma, add the defintion

$$
s_{3}(\epsilon)=E\{\phi(X, Z) \mid \delta=1\}\left\{l(\epsilon)-\frac{\epsilon}{\sigma^{2}}\right\} .
$$

Note that $s_{3}(\epsilon) \in \int$ because it satisfies the constraints in Equation A.10 and Equation A.11, namely $E\left[s_{3}(\epsilon)\right]=0$ and $E\left[\epsilon s_{3}(\epsilon)\right]=0$. The form of $v_{*}(Y, X, Z)$ can be 
rewritten as

$$
\begin{aligned}
v_{*}(Y, X, Z)= & s(\epsilon)+l(\epsilon)\left\{t^{\top} \dot{r}_{\vartheta}(X)+g(Z)\right\} \\
= & s(\epsilon)+E\{\phi(X, Z) \mid \delta=1\}\left\{l(\epsilon)-\frac{\epsilon}{\sigma^{2}}\right\} \\
& +[\phi(X, Z)-E\{\phi(X, Z) \mid \delta=1\}] l(\epsilon)+E\{\phi(X, Z) \mid \delta=1\} \frac{\epsilon}{\sigma^{2}} \\
= & s(\epsilon)+s_{3}(\epsilon)+\xi(X, Z, \epsilon) .
\end{aligned}
$$

Next I will show that $s(\epsilon)+s_{3}(\epsilon)$ belongs to the set of $s_{3}(\epsilon)$ by showing it satisfies the constraints in Equation A.10 and Equation A.11. First to show that $s(\epsilon)+s_{3}(\epsilon)$ satistifies Equation A.10.

$$
\begin{aligned}
\int\left\{s(\epsilon)+s_{3}(\epsilon)\right\} f(\epsilon) d \epsilon= & \int s(\epsilon) f(\epsilon) d \epsilon+\int s_{3}(\epsilon) f(\epsilon) d \epsilon \\
= & \int s_{3}(\epsilon) f(\epsilon) d \epsilon \\
= & \int E\{\phi(X, Z) \mid \delta=1\} l(\epsilon) f(\epsilon) d \epsilon \\
& -\int E\{\phi(X, Z) \mid \delta=1\} \epsilon / \sigma^{2} f(\epsilon) d \epsilon \\
= & E[E\{\phi(X, Z) \mid \delta=1\} l(\epsilon)]- \\
& 1 / \sigma^{2} E[E\{\phi(X, Z) \mid \delta=1\} \epsilon] \\
= & E(E[E\{\phi(X, Z) \mid \delta=1\} \mid(X, Z)] E\{l(\epsilon) \mid(X, Z)\}) \\
& -1 / \sigma^{2} E(E[E\{\phi(X, Z) \mid \delta=1\} \mid(X, Z)] E\{\epsilon \mid(X, Z)\}) \\
= & 0 .
\end{aligned}
$$


And $s(\epsilon)+s_{3}(\epsilon)$ also satisfies Equation A.11 because

$$
\begin{aligned}
\int \epsilon\left\{s(\epsilon)+s_{3}(\epsilon)\right\} f(\epsilon) d \epsilon= & \int \epsilon s(\epsilon) f(\epsilon) d \epsilon+\int \epsilon s_{3}(\epsilon) f(\epsilon) d \epsilon \\
= & \int \epsilon s_{3}(\epsilon) f(\epsilon) d \epsilon \\
= & \int E\{\phi(X, Z) \mid \delta=1\} \epsilon l(\epsilon) f(\epsilon) d \epsilon \\
& -\int E\{\phi(X, Z) \mid \delta=1\} \epsilon^{2} / \sigma^{2} f(\epsilon) d \epsilon \\
= & E[E\{\phi(X, Z) \mid \delta=1\} \epsilon l(\epsilon)] \\
& -1 / \sigma^{2} E\left[E\{\phi(X, Z) \mid \delta=1\} \epsilon^{2}\right] \\
= & E(E[E\{\phi(X, Z) \mid \delta=1\} \mid(X, Z)] E\{\epsilon l(\epsilon) \mid(X, Z)\}) \\
& -1 / \sigma^{2} E\left(E[E\{\phi(X, Z) \mid \delta=1\} \mid(X, Z)] E\left\{\epsilon^{2} \mid(X, Z)\right\}\right)
\end{aligned}
$$

Now using Lemma VI.7.

$$
\begin{aligned}
\int \epsilon\left\{s(\epsilon)+s_{3}(\epsilon)\right\} f(\epsilon) d \epsilon & =E[E\{\phi(X, Z) \mid \delta=1\}]-\sigma^{2} / \sigma^{2} E[E\{\phi(X, Z) \mid \delta=1\}] \\
& =E[E\{\phi(X, Z) \mid \delta=1\}]-E[E\{\phi(X, Z) \mid \delta=1\}] \\
& =0 .
\end{aligned}
$$

To simplify I will now refer to $s(\epsilon)+s_{3}(\epsilon)$ as simply $s_{2}(\epsilon)$ since it satisfies the conditions to be an element of the set $s(\epsilon)$. This simplifies Lemma VI.5 to $v_{*}(Y, X, Z)=s_{2}(\epsilon)+$ $\xi(X, Z, \epsilon)$. This notation is desirable because the two pieces $s_{2}(\epsilon)$ and $\delta \xi(X, Z, \epsilon)$ are orthogonal. This can be shown by finding the expected value of the product, which 
is

$$
\begin{aligned}
& E\left\{\delta s_{2}(\epsilon) \xi(X, Z, \epsilon)\right\} \\
= & E\left\{\delta s_{2}(\epsilon)\left([\phi(X, Z)-E\{\phi(X, Z) \mid \delta=1\}] l(\epsilon)+E\{\phi(X, Z) \mid \delta=1\} \frac{\epsilon}{\sigma^{2}}\right)\right\} \\
= & E\left\{\delta s_{2}(\epsilon) \phi(X, Z) l(\epsilon)\right\}-E\left\{\delta s_{2}(\epsilon) l(\epsilon)\right\} E\{\phi(X, Z) \mid \delta=1\} \\
& +\frac{1}{\sigma^{2}} E\left\{\delta s_{2}(\epsilon) \epsilon\right\} E\{\phi(X, Z) \mid \delta=1\} \\
= & E\{\delta \phi(X, Z)\} E\left\{s_{2}(\epsilon) l(\epsilon)\right\}-E(\delta) E\left\{s_{2}(\epsilon) l(\epsilon)\right\} E\{\phi(X, Z) \mid \delta=1\} \\
& +\frac{1}{\sigma^{2}} E(\delta) E\left\{s_{2}(\epsilon) \epsilon\right\} E\{\phi(X, Z) \mid \delta=1\} .
\end{aligned}
$$

Using Equation A.11 the last term is zero, and Lemma VI.8 simplifies the first term, leaving

$$
\begin{aligned}
& E\left\{\delta s_{2}(\epsilon) \xi(X, Z, \epsilon)\right\} \\
= & E\{\phi(X, Z) \mid \delta=1\} E(\delta) E\left\{s_{2}(\epsilon) l(\epsilon)\right\}-E(\delta) E\left\{s_{2}(\epsilon) l(\epsilon)\right\} E\{\phi(X, Z) \mid \delta=1\} \\
= & 0 .
\end{aligned}
$$

Thus the perturbation can be rewritten as the sum of

$$
v_{*}(Y, X, Z)=s_{2}(\epsilon)+\xi(X, Z, \epsilon)
$$

Where $s_{2}(\epsilon)$ is orthogonal to $\delta \xi(X, Z, \epsilon)$.

Lemma VI.10 The condition given in Equation A.8 can be simplified as

$$
E\{\phi(X, Z)\}=E\left[\delta\left\{s_{2 *}(\epsilon)+\xi_{*}(X, Z, \epsilon)\right\}\left\{s_{2}(\epsilon)+\xi(X, Z, \epsilon)\right\}\right]
$$

Proof: Using Lemma VI.9 into Equation A.8 the canonical gradient satisfies the 
condition

$$
\begin{aligned}
& E\left\{Y s_{2}(\epsilon)\right\}+E\{Y \xi(X, Z, \epsilon)\} \\
= & E\left[\delta\left\{s_{2 *}(\epsilon)+\xi_{*}(X, Z, \epsilon)\right\}\left\{s_{2}(\epsilon)+\xi(X, Z, \epsilon)\right\}\right] .
\end{aligned}
$$

What is left is to show $E\left\{Y s_{2}(\epsilon)\right\}+E\{Y \xi(X, Z, \epsilon)\}=E\{\phi(X, Z)\}$. This can be shown by

$$
\begin{aligned}
& E\left\{Y s_{2}(\epsilon)\right\}+E\{Y \xi(X, Z, \epsilon)\} \\
= & E\left\{Y s_{2}(\epsilon)\right\}+E\{Y([\phi(X, Z)-E\{\phi(X, Z) \mid \delta=1\}] l(\epsilon)+ \\
& \left.\left.E\{\phi(X, Z) \mid \delta=1\} \frac{\epsilon}{\sigma^{2}}\right)\right\} \\
= & E\left\{Y s_{2}(\epsilon)\right\}+E\{Y \phi(X, Z) l(\epsilon)\}-E\{Y l(\epsilon)\} E\{\phi(X, Z) \mid \delta=1\} \\
+ & \frac{1}{\sigma^{2}} E\{\phi(X, Z) \mid \delta=1\} E(Y \epsilon) .
\end{aligned}
$$

Now using the fact that $Y=r_{\vartheta}(X)+\gamma(Z)+\epsilon$,

$$
\begin{aligned}
& E\left\{Y s_{2}(\epsilon)\right\}+E\{Y \phi(X, Z) l(\epsilon)\}-E\{Y l(\epsilon)\} E\{\phi(X, Z) \mid \delta=1\} \\
& +\frac{1}{\sigma^{2}} E\{\phi(X, Z) \mid \delta=1\} E(Y \epsilon) \\
= & E\left[\left\{r_{\vartheta}(X)+\gamma(Z)+\epsilon\right\} s_{2}(\epsilon)\right]+E\left[\left\{r_{\vartheta}(X)+\gamma(Z)+\epsilon\right\} \phi(X, Z) l(\epsilon)\right] \\
& -E\left[\left\{r_{\vartheta}(X)+\gamma(Z)+\epsilon\right\} l(\epsilon)\right] E\{\phi(X, Z) \mid \delta=1\} \\
& +\frac{1}{\sigma^{2}} E\{\phi(X, Z) \mid \delta=1\} E\left[\left\{r_{\vartheta}(X)+\gamma(Z)+\epsilon\right\} \epsilon\right] \\
= & E\left[\left\{r_{\vartheta}(X)+\gamma(Z)\right\} s_{2}(\epsilon)\right]+E\left\{\epsilon s_{2}(\epsilon)\right\} \\
& +E\left[\left\{r_{\vartheta}(X)+\gamma(Z)\right\} \phi(X, Z) l(\epsilon)\right]+E\{\epsilon \phi(X, Z) l(\epsilon)\} \\
& -E\left[\left\{r_{\vartheta}(X)+\gamma(Z)\right\} l(\epsilon)\right] E\{\phi(X, Z) \mid \delta=1\}-E\{\epsilon l(\epsilon)\} E\{\phi(X, Z) \mid \delta=1\} \\
& +\frac{1}{\sigma^{2}} E\{\phi(X, Z) \mid \delta=1\} E\left[\left\{r_{\vartheta}(X)+\gamma(Z)\right\}\right]+\frac{1}{\sigma^{2}} E\left(\epsilon^{2}\right) E\{\phi(X, Z) \mid \delta=1\} .
\end{aligned}
$$

Note that since $\epsilon$ is independant of $X$ and $Z$, this equation can be simplified using 
Equation A.10 so the terms with $E\left\{s_{2}(\epsilon)\right\}=0$ and Equation A.11 so the terms with $E\left\{\epsilon s_{2}(\epsilon)\right\}=0$. In summary we have

$$
\begin{aligned}
& E\left\{Y s_{2}(\epsilon)\right\}+E\{Y \xi(X, Z, \epsilon)\} \\
= & E\left[\left\{r_{\vartheta}(X)+\gamma(Z)\right\} \phi(X, Z) l(\epsilon)\right]+E\{\epsilon \phi(X, Z) l(\epsilon)\} \\
& -E\left[\left\{r_{\vartheta}(X)+\gamma(Z)\right\} l(\epsilon)\right] E\{\phi(X, Z) \mid \delta=1\}-E\{\epsilon l(\epsilon)\} E\{\phi(X, Z) \mid \delta=1\} \\
& +\frac{1}{\sigma^{2}} E\{\phi(X, Z) \mid \delta=1\} E\left[\left\{r_{\vartheta}(X)+\gamma(Z)\right\} \epsilon\right]+\frac{1}{\sigma^{2}} E\left(\epsilon^{2}\right) E\{\phi(X, Z) \mid \delta=1\} .
\end{aligned}
$$

Now using the fact that $\epsilon$ is independant of $X$ and $Z$ and the fact that $E(\epsilon)=$ $E\{l(\epsilon)\}=0$ and Lemma VI.7 which states $E\{\epsilon l(\epsilon)\}=1$ we can simplify the rest of the equation to be

$$
\begin{aligned}
E\left\{Y s_{2}(\epsilon)\right\}+E\{Y \xi(X, Z, \epsilon)\}= & E\{\phi(X, Z)\}-E\{\phi(X, Z) \mid \delta=1\} \\
& +E\{\phi(X, Z) \mid \delta=1\} \\
= & E\{\phi(X, Z)\} .
\end{aligned}
$$

This concludes the simplification of the first half of the equation, so the final form is

$$
E\{\phi(X, Z)\}=E\left[\delta\left\{s_{2 *}(\epsilon)+\xi_{*}(X, Z, \epsilon)\right\}\left\{s_{2}(\epsilon)+\xi(X, Z, \epsilon)\right\}\right] .
$$

Lemma VI.11 Using the notation given, $s_{2 *}(\epsilon)=0$ which means

$$
v_{*}(Y, X, Z)=\xi_{*}(X, Z, \epsilon)
$$

Proof: Lemma VI.10 holds for any $t$ and $g(Z)$, so it holds for $t=0$ and $g(Z)=0$. 
In this case $\phi(X, Z)=0$, and

$$
\xi(X, Z, \epsilon)=[\phi(X, Z)-E\{\phi(X, Z) \mid \delta=1\}] l(\epsilon)+E\{\phi(X, Z) \mid \delta=1\} \frac{\epsilon}{\sigma^{2}}=0
$$

So Lemma VI.10 in this case means

$$
\begin{aligned}
0= & E\left[\delta\left\{s_{2 *}(\epsilon)+\xi_{*}(X, Z, \epsilon)\right\} s_{2}(\epsilon)\right] \\
= & E\left\{\delta s_{2 *}(\epsilon) s_{2}(\epsilon)+\delta s_{2}(\epsilon) \xi_{*}(X, Z, \epsilon)\right\} \\
= & E\left\{\delta s_{2 *}(\epsilon) s_{2}(\epsilon)\right\} \\
& +E\left(\delta s_{2}(\epsilon)\left[\phi_{*}(X, Z) l(\epsilon)-E\left\{\phi_{*}(X, Z) \mid \delta=1\right\} l(\epsilon)+\frac{\epsilon}{\sigma^{2}} E\left\{\phi_{*}(X, Z) \mid \delta=1\right\}\right]\right)
\end{aligned}
$$

This is simplified in a similar manner to Lemma VI.6 where the expected value of a function of $(X, Z, \delta)$ times $\epsilon$ is zero.

$$
0=E\left\{\delta s_{2 *}(\epsilon) s_{2}(\epsilon)\right\}+E\left\{\delta s_{2}(\epsilon) \phi_{*}(X, Z) l(\epsilon)\right\}-E\left[\delta s_{2}(\epsilon) E\left\{\phi_{*}(X, Z) \mid \delta=1\right\} l(\epsilon)\right]
$$

Then using Lemma VI.8

$$
\begin{aligned}
0= & E\left\{\delta s_{2 *}(\epsilon) s_{2}(\epsilon)\right\}+E(\delta) E\left\{s_{2}(\epsilon) \phi_{*}(X, Z) l(\epsilon) \mid \delta=1\right\} \\
& -E(\delta) E\left\{s_{2}(\epsilon) l(\epsilon) \mid \delta=1\right\} E\{\phi(X, Z) \mid \delta=1\} \\
= & E\left\{\delta s_{2 *}(\epsilon) s_{2}(\epsilon)\right\}+E(\delta) E\left\{s_{2}(\epsilon) l(\epsilon) \mid \delta=1\right\} E\{\phi(X, Z) \mid \delta=1\} \\
& -E(\delta) E\left\{s_{2}(\epsilon) l(\epsilon) \mid \delta=1\right\} E\{\phi(X, Z) \mid \delta=1\} \\
= & E\left\{\delta s_{2 *}(\epsilon) s_{2}(\epsilon)\right\} .
\end{aligned}
$$

The solution to this is $s_{2 *}(\epsilon)=0$, and $v_{*}(Y, X, Z)=\xi_{*}(X, Z, \epsilon)$.

Corollary VI.12 Lemma VI.10 can be simplified to

$$
E\{\phi(X, Z)\}=E\left\{\delta \xi_{*}(X, Z, \epsilon) \xi(X, Z, \epsilon)\right\} .
$$


Proof: From Lemma VI.11 the equation given by Lemma VI.10 becomes

$$
\begin{aligned}
E\{\phi(X, Z)\} & =E\left[\delta \xi_{*}(X, Z, \epsilon)\left\{s_{2}(\epsilon)+\xi(X, Z, \epsilon)\right\}\right] \\
& =E\left\{\delta \xi_{*}(X, Z, \epsilon) s_{2}(\epsilon)\right\}+E\left\{\delta \xi_{*}(X, Z, \epsilon) \xi(X, Z, \epsilon)\right\} .
\end{aligned}
$$

To get the form desired it is sufficient to show $E\left\{\delta \xi_{*}(X, Z, \epsilon) s_{2}(\epsilon)\right\}=0$.

$$
\begin{aligned}
& E\left\{\delta \xi_{*}(X, Z, \epsilon) s_{2}(\epsilon)\right\} \\
= & E\left(\delta s_{2}(\epsilon)\left[\phi_{*}(X, Z) l(\epsilon)-E\left\{\phi_{*}(X, Z) \mid \delta=1\right\} l(\epsilon)+\frac{\epsilon}{\sigma^{2}} E\left\{\phi_{*}(X, Z) \mid \delta=1\right\}\right]\right) \\
= & E\left\{\delta s_{2}(\epsilon) \phi_{*}(X, Z) l(\epsilon)\right\}-E\left\{\phi_{*}(X, Z) \mid \delta=1\right\} E\left\{\delta s_{2}(\epsilon) l(\epsilon)\right\} \\
& +\frac{1}{\sigma^{2}} E\left\{\phi_{*}(X, Z) \mid \delta=1\right\} E\left\{\delta s_{2}(\epsilon) \epsilon\right\} .
\end{aligned}
$$

The last term is zero by Equation A.11, the second term can be simplified by LemmaVI.8

$$
\begin{aligned}
E\left\{\delta \xi_{*}(X, Z, \epsilon) s_{2}(\epsilon)\right\} & =E\left\{\delta \phi_{*}(X, Z)\right\} E\left\{s_{2}(\epsilon) l(\epsilon)\right\}-\frac{E(\delta)}{E(\delta)} E\left\{\delta \phi_{*}(X, Z)\right\} E\left\{s_{2}(\epsilon) l(\epsilon)\right\} \\
& =0 .
\end{aligned}
$$

This finishes the proof.

5. Solving for the canonical gradient

The next Lemma will help simplify the canonical gradient further.

Lemma VI.13 For the model described above $E\left\{\delta \phi_{*}(X, Z)\right\}=\sigma^{2}$. 
Proof: Starting with Corollary VI.12,

$$
\begin{aligned}
E\{\phi(X, Z)\}= & E\left\{\delta \xi_{*}(X, Z, \epsilon) \xi(X, Z, \epsilon)\right\} \\
= & E\left(\delta \xi_{*}(X, Z, \epsilon)[\phi(X, Z) l(\epsilon)-E\{\phi(X, Z) \mid \delta=1\} l(\epsilon)\right. \\
& \left.\left.+\frac{\epsilon}{\sigma^{2}} E\{\phi(X, Z) \mid \delta=1\}\right]\right) \\
= & E\left\{\delta \xi_{*}(X, Z, \epsilon) \phi(X, Z) l(\epsilon)\right\}-E\left\{\delta \xi_{*}(X, Z, \epsilon) l(\epsilon)\right\} E\{\phi(X, Z) \mid \delta=1\} \\
& +\frac{1}{\sigma^{2}} E\left\{\delta \xi_{*}(X, Z, \epsilon) \epsilon\right\} E\{\phi(X, Z) \mid \delta=1\} .
\end{aligned}
$$

By Lemma VI.8

$$
\begin{aligned}
E\{\phi(X, Z)\}= & E\left\{\delta \xi_{*}(X, Z, \epsilon) \phi(X, Z) l(\epsilon)\right\}-E\left\{\delta \xi_{*}(X, Z, \epsilon) l(\epsilon)\right\} \frac{E\{\delta \phi(X, Z)\}}{E(\delta)} \\
& +\frac{1}{\sigma^{2}} E\left\{\delta \xi_{*}(X, Z, \epsilon) \epsilon\right\} \frac{E\{\delta \phi(X, Z)\}}{E(\delta)} \\
= & E\left(\left[\delta \xi_{*}(X, Z, \epsilon) l(\epsilon)-\delta \frac{E\left\{\delta \xi_{*}(X, Z, \epsilon) l(\epsilon)\right\}}{E(\delta)}\right.\right. \\
& \left.\left.+\delta \frac{E\left\{\delta \xi_{*}(X, Z, \epsilon) \epsilon\right\}}{\sigma^{2} E(\delta)}\right] \phi(X, Z)\right) .
\end{aligned}
$$

By setting the equation to zero the form is

$$
\begin{aligned}
0= & E\left(\left[\delta \xi_{*}(X, Z, \epsilon) l(\epsilon)-\delta \frac{E\left\{\delta \xi_{*}(X, Z, \epsilon) l(\epsilon)\right\}}{E(\delta)}\right.\right. \\
& \left.\left.+\delta \frac{E\left\{\delta \xi_{*}(X, Z, \epsilon) \epsilon\right\}}{\sigma^{2} E(\delta)}-1\right] \phi(X, Z)\right) .
\end{aligned}
$$

Since this must be true for any $\phi(X, Z)$ including $\phi(X, Z)=1$,

$$
\begin{aligned}
0 & =E\left[\delta \xi_{*}(X, Z, \epsilon) l(\epsilon)-\delta \frac{E\left\{\delta \xi_{*}(X, Z, \epsilon) l(\epsilon)\right\}}{E(\delta)}+\delta \frac{E\left\{\delta \xi_{*}(X, Z, \epsilon) \epsilon\right\}}{\sigma^{2} E(\delta)}-1\right] \\
& =E\left\{\delta \xi_{*}(X, Z, \epsilon) l(\epsilon)\right\}-\frac{E(\delta)}{E(\delta)} E\left\{\delta \xi_{*}(X, Z, \epsilon) l(\epsilon)\right\}+\frac{E(\delta)}{\sigma^{2} E(\delta)} E\left\{\delta \xi_{*}(X, Z, \epsilon) \epsilon\right\}-1 \\
& =\frac{1}{\sigma^{2}} E\left\{\delta \xi_{*}(X, Z, \epsilon) \epsilon\right\}-1 .
\end{aligned}
$$


Which means

$$
\begin{aligned}
1= & \frac{1}{\sigma^{2}} E\left(\delta \epsilon\left[\phi_{*}(X, Z) l(\epsilon)-E\left\{\phi_{*}(X, Z) \mid \delta=1\right\} l(\epsilon)+\frac{\epsilon}{\sigma^{2}} E\left\{\phi_{*}(X, Z) \mid \delta=1\right\}\right]\right) \\
= & \frac{1}{\sigma^{2}} E\left\{\delta \epsilon \phi_{*}(X, Z) l(\epsilon)\right\}-\frac{1}{\sigma^{2}} E\{\delta \epsilon l(\epsilon)\} E\left\{\phi_{*}(X, Z) \mid \delta=1\right\} \\
& +\frac{1}{\sigma^{2}} E\left\{\delta \frac{\epsilon^{2}}{\sigma^{2}}\right\} E\left\{\phi_{*}(X, Z) \mid \delta=1\right\} .
\end{aligned}
$$

By Lemma VI.7 and using Lemma VI.8,

$$
\begin{aligned}
\sigma^{2} & =E\left\{\delta \phi_{*}(X, Z)\right\}-E(\delta) E\left\{\phi_{*}(X, Z) \mid \delta=1\right\}+E(\delta) E\left\{\phi_{*}(X, Z) \mid \delta=1\right\} \\
& =E\left\{\delta \phi_{*}(X, Z)\right\}
\end{aligned}
$$

One benefit from this lemma is in the simplification of $\xi_{*}(X, Z, \epsilon)$ as shown in the following Corollary.

Corollary VI.14 Using the notation given

$$
\xi_{*}(X, Z, \epsilon)=\phi_{*}(X, Z) l(\epsilon)-\frac{\sigma^{2}}{E(\delta)} l(\epsilon)+\frac{\epsilon}{E(\delta)} .
$$

PROOF: From Lemma VI.8 substituting in Lemma VI.13.

$$
E\left\{\phi_{*}(X, Z) \mid \delta=1\right\}=\frac{\sigma^{2}}{E(\delta)} .
$$

Which means

$$
\begin{aligned}
\xi_{*}(X, Z, \epsilon) & =\phi_{*}(X, Z) l(\epsilon)-E\left\{\phi_{*}(X, Z) \mid \delta=1\right\} l(\epsilon)-\frac{\epsilon}{\sigma^{2}} E\left\{\phi_{*}(X, Z) \mid \delta=1\right\} \\
& =\phi_{*}(X, Z) l(\epsilon)-\frac{\sigma^{2}}{E(\delta)} l(\epsilon)+\frac{\epsilon}{E(\delta)} .
\end{aligned}
$$


The simplification of Equation A.15 using Lemma VI.13 will be easier to follow if the expected values are solved individually. This will be done in the following three lemmas. Define $\mathbb{I}$ as the Fischer Information for $\epsilon$.

Lemma VI.15 Using the notation above

$$
E\left\{\delta l(\epsilon) \xi_{*}(X, Z, \epsilon)\right\}=1
$$

\section{PROOF:}

$$
\begin{aligned}
E\left\{\delta l(\epsilon) \xi_{*}(X, Z, \epsilon)\right\} & =E\left[\delta l(\epsilon)\left\{\phi_{*}(X, Z) l(\epsilon)-\frac{\sigma^{2}}{E(\delta)} l(\epsilon)+\frac{\epsilon}{E(\delta)}\right\}\right] \\
& =E\left\{\delta \phi_{*}(X, Z)\right\} E\left\{l^{2}(\epsilon)\right\}-\frac{\sigma^{2}}{E(\delta)} E(\delta) E\left\{l^{2}(\epsilon)\right\}+\frac{E(\delta)}{E(\delta)} E\{\epsilon l(\epsilon)\} \\
& =E\left\{\delta \phi_{*}(X, Z)\right\} \mathbb{I}-\frac{E(\delta)}{E(\delta)} \sigma^{2} \mathbb{I}+1 .
\end{aligned}
$$

By Lemma VI.13

$$
\begin{aligned}
E\left\{\delta l(\epsilon) \xi_{*}(X, Z, \epsilon)\right\} & =\sigma^{2} \mathbb{I}-\sigma^{2} \mathbb{I}+1 \\
& =1 .
\end{aligned}
$$

Lemma VI.16 Using the notation above

$$
E\left\{\delta \epsilon \xi_{*}(X, Z, \epsilon)\right\}=\sigma^{2}
$$




\section{PROOF:}

$$
\begin{aligned}
E\left\{\delta \epsilon \xi_{*}(X, Z, \epsilon)\right\} & =E\left[\delta \epsilon\left\{\phi_{*}(X, Z) l(\epsilon)-\frac{\sigma^{2}}{E(\delta)} l(\epsilon)+\frac{\epsilon}{E(\delta)}\right\}\right] \\
& =E\left\{\delta \phi_{*}(X, Z)\right\} E\{\epsilon l(\epsilon)\}-\frac{\sigma^{2}}{E(\delta)} E(\delta) E\{\epsilon l(\epsilon)\}+\frac{E(\delta)}{E(\delta)} E\left(\epsilon^{2}\right) .
\end{aligned}
$$

By Lemma VI.7 and Lemma VI.13

$$
\begin{aligned}
E\left\{\delta \epsilon \xi_{*}(X, Z, \epsilon)\right\} & =E\left\{\delta \phi_{*}(X, Z)\right\}-\sigma^{2}+\sigma^{2} \\
& =\sigma^{2}
\end{aligned}
$$

Lemma VI.17 Using the notation above

$$
\begin{aligned}
E\left\{\delta \phi(X, Z) l(\epsilon) \xi_{*}(X, Z, \epsilon)\right\}= & E\left\{\delta \phi(X, Z) \phi_{*}(X, Z)\right\} \mathbb{I}-\frac{\sigma^{2}}{E(\delta)} E\{\delta \phi(X, Z)\} \mathbb{I} \\
& +\frac{E\{\delta \phi(X, Z)\}}{E(\delta)} .
\end{aligned}
$$

PROOF:

$$
\begin{aligned}
& E\left\{\delta \phi(X, Z) l(\epsilon) \xi_{*}(X, Z, \epsilon)\right\} \\
= & E\left[\delta \phi(X, Z) l(\epsilon)\left\{\phi_{*}(X, Z) l(\epsilon)-\frac{\sigma^{2}}{E(\delta)} l(\epsilon)+\frac{\epsilon}{E[\delta]}\right\}\right] \\
= & E\left\{\delta \phi(X, Z) \phi_{*}(X, Z)\right\} E\left\{l^{2}(\epsilon)\right\}-\frac{\sigma^{2}}{E(\delta)} E\{\delta \phi(X, Z)\} E\left\{l^{2}(\epsilon)\right\} \\
& +\frac{E\{\delta \phi(X, Z)\}}{E(\delta)} E\{\epsilon l(\epsilon)\} \\
= & E\left\{\delta \phi(X, Z) \phi_{*}(X, Z)\right\} \mathbb{I}-\frac{\sigma^{2}}{E(\delta)} E\{\delta \phi(X, Z)\} \mathbb{I}+\frac{E\{\delta \phi(X, Z)\}}{E(\delta)} .
\end{aligned}
$$

Now an important lemma to solve for $g_{*}(Z)$ in terms of $t_{*}$. 
Lemma VI.18 Using the notation given

$$
g_{*}(Z)=\frac{\sigma^{2}}{E(\delta)}-\frac{1}{E(\delta) \mathbb{I}}+\frac{1}{E(\delta \mid Z) \mathbb{I}}-t_{*}^{\top} \frac{E\left\{\delta \dot{r}_{\vartheta}(X) \mid Z\right\}}{E(\delta \mid Z)} .
$$

PROOF: Begin with Equation A.15

$$
\begin{aligned}
0= & E\left(\left[\delta \xi_{*}(X, Z, \epsilon) l(\epsilon)-\delta \frac{E\left\{\delta \xi_{*}(X, Z, \epsilon) l(\epsilon)\right\}}{E(\delta)}\right.\right. \\
& \left.\left.+\delta \frac{E\left\{\delta \xi_{*}(X, Z, \epsilon) \epsilon\right\}}{\sigma^{2} E(\delta)}-1\right] \phi(X, Z)\right) .
\end{aligned}
$$

Using Lemma VI.15 and Lemma VI.16,

$$
\begin{aligned}
0 & =E\left[\left\{\delta \xi_{*}(X, Z, \epsilon) l(\epsilon)-\delta \frac{1}{E(\delta)}+\delta \frac{\sigma^{2}}{\sigma^{2} E(\delta)}-1\right\} \phi(X, Z)\right] \\
& =E\left[\left\{\delta \xi_{*}(X, Z, \epsilon) l(\epsilon)-1\right\} \phi(X, Z)\right] .
\end{aligned}
$$

Then using Lemma VI.17,

$$
\begin{aligned}
0 & =E\left\{\delta \phi(X, Z) \phi_{*}(X, Z)\right\} \mathbb{I}-\frac{\sigma^{2}}{E(\delta)} E\{\delta \phi(X, Z)\} \mathbb{I}+\frac{E\{\delta \phi(X, Z)\}}{E(\delta)}-E\{\phi(X, Z)\} \\
& =E\left[\left\{\delta \phi_{*}(X, Z) \mathbb{I}-\frac{\sigma^{2} \delta \mathbb{I}}{E(\delta)}+\frac{\delta}{E(\delta)}-1\right\} \phi(X, Z)\right] .
\end{aligned}
$$

This is true for any $t$, so set $t=0$. In this case $\phi(X, Z)=g(Z)$. The equation then becomes

$$
0=E\left[\left\{\delta \phi_{*}(X, Z) \mathbb{I}-\frac{\sigma^{2} \delta \mathbb{I}}{E(\delta)}+\frac{\delta}{E(\delta)}-1\right\} g(Z)\right]
$$

Using the Law of Iterated Expectations I will rewrite this equation so that $\phi(X, Z)$ is being multiplied by a function of $Z$.

$$
0=E\left[\left\{E\left\{\delta \phi_{*}(X, Z) \mid Z\right\} \mathbb{I}-\frac{\sigma^{2} E(\delta \mid Z) \mathbb{I}}{E(\delta)}+\frac{E(\delta \mid Z)}{E(\delta)}-1\right\} g(Z)\right] .
$$


To simplify the notation momentarily let

$$
M(Z)=E\left\{\delta \phi_{*}(X, Z) \mid Z\right\} \mathbb{I}-\frac{\sigma^{2} E(\delta \mid Z) \mathbb{I}}{E(\delta)}+\frac{E(\delta \mid Z)}{E(\delta)}-1
$$

so that Equation A.17 becomes

$$
0=E\{M(Z) g(Z)\}
$$

Note also that

$$
\begin{aligned}
E[M(Z)] & =E\left[E\left\{\delta \phi_{*}(X, Z) \mid Z\right\} \mathbb{I}-\frac{\sigma^{2} E(\delta \mid Z) \mathbb{I}}{E(\delta)}+\frac{E(\delta \mid Z)}{E(\delta)}-1\right] \\
& =E\left\{\delta \phi_{*}(X, Z)\right\} \mathbb{I}-\frac{\sigma^{2} E(\delta) \mathbb{I}}{E(\delta)}+\frac{E(\delta)}{E(\delta)}-1 \\
& =E\left\{\delta \phi_{*}(X, Z)\right\} \mathbb{I}-\sigma^{2} \mathbb{I} \\
& =0 .
\end{aligned}
$$

This is true for any $g(Z)$, so let $g(Z)=E\{g(Z)\}+g_{0}(Z)$ where $E\{g(Z)\}$ is a constant, and $E\left\{g_{0}(Z)\right\}=0$. This changes Equation A.18 to

$$
\begin{aligned}
0 & =E\left(M(Z)\left[E\{g(Z)\}+g_{0}(Z)\right]\right) \\
& =E[M(Z) E\{g(Z)\}]+E\left\{M(Z) g_{0}(Z)\right\} \\
& =E\{g(Z)\} E\{M(Z)\}+E\left\{M(Z) g_{0}(Z)\right\} \\
& =E\left\{M(Z) g_{0}(Z)\right\}
\end{aligned}
$$

This is true for all $g_{0}(Z)$, which is not a constant, then $M(Z)$ must equal zero.

$$
\begin{aligned}
0 & =M(Z) \\
& =E\left\{\delta \phi_{*}(X, Z) \mid Z\right\} \mathbb{I}-\frac{\sigma^{2} E(\delta \mid Z) \mathbb{I}}{E(\delta)}+\frac{E(\delta \mid Z)}{E(\delta)}-1
\end{aligned}
$$


Then substituting $\phi_{*}(X, Z)=t_{*}^{\top} \dot{r}_{\vartheta}(X)+g_{*}(Z)$

$$
\begin{aligned}
0 & =E\left[\delta\left\{t_{*}^{\top} \dot{r}_{\vartheta}(X)+g_{*}(Z)\right\} \mid Z\right] \mathbb{I}-\frac{\sigma^{2} E(\delta \mid Z) \mathbb{I}}{E(\delta)}+\frac{E(\delta \mid Z)}{E(\delta)}-1 \\
& =t_{*}^{\top} E\left\{\delta \dot{r}_{\vartheta}(X) \mid Z\right\} \mathbb{I}+g_{*}(Z) E(\delta \mid Z) \mathbb{I}-\frac{\sigma^{2} E(\delta \mid Z) \mathbb{I}}{E(\delta)}+\frac{E(\delta \mid Z)}{E(\delta)}-1 .
\end{aligned}
$$

Which means

$$
g_{*}(Z)=\frac{\sigma^{2}}{E(\delta)}-\frac{1}{E(\delta) \mathbb{I}}+\frac{1}{E(\delta \mid Z) \mathbb{I}}-t_{*}^{\top} \frac{E\left\{\delta \dot{r}_{\vartheta}(X) \mid Z\right\}}{E(\delta \mid Z)}
$$

Now the solution for $t_{*}$ can be found.

Lemma VI.19 Using the given notation

$$
\begin{aligned}
t_{*}= & \frac{1}{\mathbb{I}}\left(E\left\{\delta \dot{r}_{\vartheta}(X) \dot{r}_{\vartheta}(X)^{\top}\right\}-E\left[\frac{E\left\{\delta \dot{r}_{\vartheta}(X) \mid Z\right\} E\left\{\delta \dot{r}_{\vartheta}(X) \mid Z\right\}^{\top}}{E(\delta \mid Z)}\right]\right)^{-1} \\
& {\left[E\left\{\dot{r}_{\vartheta}(X)\right\}-\frac{E\left\{\delta \dot{r}_{\vartheta}(X) \mid Z\right\}}{E(\delta \mid Z)}\right] . }
\end{aligned}
$$

Proof: Begin with Equation A.16.

$$
0=E\left[\left\{\delta \phi_{*}(X, Z) \mathbb{I}-\frac{\sigma^{2} \delta \mathbb{I}}{E(\delta)}+\frac{\delta}{E(\delta)}-1\right\} \phi(X, Z)\right]
$$

which is true for any $g(Z)$, so set $g(Z)=0$, which means $\phi(X, Z)=t^{\top} \dot{r}_{\vartheta}(X)$, and

$$
\begin{aligned}
0 & =E\left[\left\{\delta \phi_{*}(X, Z) \mathbb{I}-\frac{\sigma^{2} \delta \mathbb{I}}{E(\delta)}+\frac{\delta}{E(\delta)}-1\right\} t^{\top} \dot{r}_{\vartheta}(X)\right] \\
& =E\left\{\delta \phi_{*}(X, Z) \mathbb{I} t^{\top} \dot{r}_{\vartheta}(X)-\frac{\sigma^{2} \delta \mathbb{I} t^{\top} \dot{r}_{\vartheta}(X)}{E(\delta)}+\frac{\delta t^{\top} \dot{r}_{\vartheta}(X)}{E(\delta)}-t^{\top} \dot{r}_{\vartheta}(X)\right\} .
\end{aligned}
$$


Then substituting $\phi_{*}(X, Z)=t_{*}^{\top} \dot{r}_{\vartheta}(X)+g_{*}(Z)$,

$$
\begin{aligned}
0= & E\left[\delta\left\{t_{*}^{\top} \dot{r}_{\vartheta}(X)+g_{*}(Z)\right\} \mathbb{I} t^{\top} \dot{r}_{\vartheta}(X)\right. \\
& \left.-\frac{\sigma^{2} \delta \mathbb{I} t^{\top} \dot{r}_{\vartheta}(X)}{E(\delta)}+\frac{\delta t^{\top} \dot{r}_{\vartheta}(X)}{E(\delta)}-t^{\top} \dot{r}_{\vartheta}(X)\right] \\
= & E\left\{\delta t_{*}^{\top} \dot{r}_{\vartheta}(X) \mathbb{I} t^{\top} \dot{r}_{\vartheta}(X)+\delta g_{*}(Z) \mathbb{I} t^{\top} \dot{r}_{\vartheta}(X)-\frac{\sigma^{2} \delta \mathbb{I} t^{\top} \dot{r}_{\vartheta}(X)}{E(\delta)}\right. \\
& \left.+\frac{\delta t^{\top} \dot{r}_{\vartheta}(X)}{E(\delta)}-t^{\top} \dot{r}_{\vartheta}(X)\right\} .
\end{aligned}
$$

Using Lemma VI.18,

$$
\begin{aligned}
0= & E\left(\delta t_{*}^{\top} \dot{r}_{\vartheta}(X) \mathbb{I} t^{\top} \dot{r}_{\vartheta}(X)\right. \\
& +\delta\left[\frac{\sigma^{2}}{E(\delta)}-\frac{1}{E(\delta) \mathbb{I}}+\frac{1}{E(\delta \mid Z) \mathbb{I}}-t_{*}^{\top} \frac{E\left\{\delta \dot{r}_{\vartheta}(X) \mid Z\right\}}{E(\delta \mid Z)}\right] \mathbb{I} t^{\top} \dot{r}_{\vartheta}(X) \\
& \left.-\frac{\sigma^{2} \delta \mathbb{I} t^{\top} \dot{r}_{\vartheta}(X)}{E(\delta)}+\frac{\delta t^{\top} \dot{r}_{\vartheta}(X)}{E(\delta)}-t^{\top} \dot{r}_{\vartheta}(X)\right) \\
= & E\left[\delta t_{*}^{\top} \dot{r}_{\vartheta}(X) \mathbb{I} t^{\top} \dot{r}_{\vartheta}(X)+\frac{\delta \mathbb{I} t^{\top} \dot{r}_{\vartheta}(X) \sigma^{2}}{E(\delta)}-\frac{\delta \mathbb{I} t^{\top} \dot{r}_{\vartheta}(X)}{E(\delta) \mathbb{I}}+\frac{\delta \mathbb{I} t^{\top} \dot{r}_{\vartheta}(X)}{E(\delta \mid Z) \mathbb{I}}\right. \\
& \left.-\delta \mathbb{I} t^{\top} \dot{r}_{\vartheta}(X) t_{*}^{\top} \frac{E\left\{\delta \dot{r}_{\vartheta}(X) \mid Z\right\}}{E(\delta \mid Z)}-\frac{\sigma^{2} \delta \mathbb{I} t^{\top} \dot{r}_{\vartheta}(X)}{E(\delta)}+\frac{\delta t^{\top} \dot{r}_{\vartheta}(X)}{E(\delta)}-t^{\top} \dot{r}_{\vartheta}(X)\right] \\
= & \mathbb{I} t^{\top} E\left\{\delta \dot{r}_{\vartheta}(X) \dot{r}_{\vartheta}(X)^{\top}\right\} t_{*}+\sigma^{2} \mathbb{I} t^{\top} \frac{E\left\{\delta \dot{r}_{\vartheta}(X)\right\}}{E(\delta)} \\
& -t^{\top} \frac{E\left\{\delta \dot{r}_{\vartheta}(X)\right\}}{E(\delta)}+t^{\top} E\left\{\frac{\delta \dot{r}_{\vartheta}(X)}{E(\delta \mid Z)}\right\} \\
& -\mathbb{I} t^{\top} E\left[\delta \dot{r}_{\vartheta}(X) \frac{E\left\{\delta \dot{r}_{\vartheta}(X) \mid Z\right\}^{\top}}{E(\delta \mid Z)}\right] t_{*}-\sigma^{2} \mathbb{I} t^{\top} \frac{E\left\{\delta \dot{r}_{\vartheta}(X)\right\}}{E(\delta)}+t^{\top} \frac{E\left\{\delta \dot{r}_{\vartheta}(X)\right\}}{E(\delta)} \\
& -t^{\top} E\left\{\dot{r}_{\vartheta}(X)\right\} .
\end{aligned}
$$


Then factoring out the constants $t$ and $t_{*}$,

$$
\begin{aligned}
0= & t^{\top}\left\{\sigma^{2} \mathbb{I} \frac{E\left\{\delta \dot{r}_{\vartheta}(X)\right\}}{E(\delta)}-\frac{E\left\{\delta \dot{r}_{\vartheta}(X)\right\}}{E(\delta)}+E\left[\frac{E\left\{\delta \dot{r}_{\vartheta}(X) \mid Z\right\}}{E(\delta \mid Z)}\right]-\sigma^{2} \mathbb{I} \frac{E\left\{\delta \dot{r}_{\vartheta}(X)\right\}}{E(\delta)}\right. \\
& +\frac{E\left\{\delta \dot{r}_{\vartheta}(X)\right\}}{E(\delta)}-E\left\{\dot{r}_{\vartheta}(X)\right\} \\
& \left.+\left(\mathbb{I} E\left\{\delta \dot{r}_{\vartheta}(X) \dot{r}_{\vartheta}(X)^{\top}\right\}-\mathbb{I} E\left[\frac{E\left\{\delta \dot{r}_{\vartheta}(X) \mid Z\right\} E\left\{\delta \dot{r}_{\vartheta}(X) \mid Z\right\}^{\top}}{E\{\delta \mid Z\}}\right]\right) t_{*}\right\} \\
= & t^{\top}\left\{E\left[\frac{E\left\{\delta \dot{r}_{\vartheta}(X) \mid Z\right\}}{E(\delta \mid Z)}\right]-E\left\{\dot{r}_{\vartheta}(X)\right\}\right. \\
& \left.+\left(\mathbb{I} E\left\{\delta \dot{r}_{\vartheta}(X) \dot{r}_{\vartheta}(X)^{\top}\right\}-\mathbb{I} E\left[\frac{E\left\{\delta \dot{r}_{\vartheta}(X) \mid Z\right\} E\left\{\delta \dot{r}_{\vartheta}(X) \mid Z\right\}^{\top}}{E(\delta \mid Z)}\right]\right) t_{*}\right\} .
\end{aligned}
$$

Since this is true for any $t$ let $t=1$. Then

$$
\begin{aligned}
0= & E\left[\frac{E\left\{\delta \dot{r}_{\vartheta}(X) \mid Z\right\}}{E(\delta \mid Z)}\right]-E\left\{\dot{r}_{\vartheta}(X)\right\} \\
& +\left(\mathbb{I} E\left\{\delta \dot{r}_{\vartheta}(X) \dot{r}_{\vartheta}(X)^{\top}\right\}-\mathbb{I} E\left[\frac{E\left\{\delta \dot{r}_{\vartheta}(X) \mid Z\right\} E\left\{\delta \dot{r}_{\vartheta}(X) \mid Z\right\}^{\top}}{E(\delta \mid Z)}\right]\right) t_{*} .
\end{aligned}
$$

This means

$$
\begin{aligned}
t_{*}= & \frac{1}{\mathbb{I}}\left(E\left\{\delta \dot{r}_{\vartheta}(X) \dot{r}_{\vartheta}(X)^{\top}\right\}-E\left[\frac{E\left\{\delta \dot{r}_{\vartheta}(X) \mid Z\right\} E\left\{\delta \dot{r}_{\vartheta}(X) \mid Z\right\}^{\top}}{E(\delta \mid Z)}\right]\right)^{-1} \\
& \left(E\left\{\dot{r}_{\vartheta}(X)\right\}-E\left[\frac{E\left\{\delta \dot{r}_{\vartheta}(X) \mid Z\right\}}{E(\delta \mid Z)}\right]\right) .
\end{aligned}
$$

Lemma VI.20 Using the notation given

$$
\begin{aligned}
v_{*}(Y, X, Z)= & \frac{\epsilon}{E(\delta)}-\frac{l(\epsilon)}{E(\delta) \mathbb{I}}+\frac{l(\epsilon)}{E(\delta \mid Z) \mathbb{I}}- \\
& \frac{l(\epsilon)}{\mathbb{I}}\left(E\left\{\dot{r}_{\vartheta}(X)^{\top}\right\}-E\left[\frac{E\left\{\delta \dot{r}_{\vartheta}(X) \mid Z\right\}}{E(\delta \mid Z)}\right]\right) \\
& \left(E\left\{\delta \dot{r}_{\vartheta}(X) \dot{r}_{\vartheta}(X)^{\top}\right\}-E\left[\frac{E\left\{\delta \dot{r}_{\vartheta}(X) \mid Z\right\} E\left\{\delta \dot{r}_{\vartheta}(X) \mid Z\right\}^{\top}}{E(\delta \mid Z)}\right]\right)^{-1} \\
& {\left[\dot{r}_{\vartheta}(X)-\frac{E\left\{\delta \dot{r}_{\vartheta}(X) \mid Z\right\}}{E(\delta \mid Z)}\right] }
\end{aligned}
$$


PROOF: The proof requires plugging $t$ and $g(Z)$ into Lemma VI.11. Using LemmaVI.19 with Lemma VI.18

$$
\begin{aligned}
g_{*}(Z)= & \frac{\sigma^{2}}{E(\delta)}-\frac{1}{E(\delta) \mathbb{I}}+\frac{1}{E(\delta \mid Z) \mathbb{I}} \\
& -\left\{\frac{1}{\mathbb{I}}\left(E\left\{\delta \dot{r}_{\vartheta}(X) \dot{r}_{\vartheta}(X)^{\top}\right\}-E\left[\frac{E\left\{\delta \dot{r}_{\vartheta}(X) \mid Z\right\} E\left\{\delta \dot{r}_{\vartheta}(X) \mid Z\right\}^{\top}}{E(\delta \mid Z)}\right]\right)^{-1}\right. \\
& \left.\left(E\left\{\dot{r}_{\vartheta}(X)\right\}-E\left[\frac{E\left\{\delta \dot{r}_{\vartheta}(X) \mid Z\right\}}{E(\delta \mid Z)}\right]\right)\right\}^{\top} \frac{E\left\{\delta \dot{r}_{\vartheta}(X) \mid Z\right\}}{E(\delta \mid Z)} \\
= & \frac{\sigma^{2}}{E(\delta)}-\frac{1}{E(\delta) \mathbb{I}}+\frac{1}{E(\delta \mid Z) \mathbb{I}} \\
& -\frac{1}{\mathbb{I}}\left(E\left\{\dot{r}_{\vartheta}(X)^{\top}\right\}-E\left[\frac{E\left\{\delta \dot{r}_{\vartheta}(X) \mid Z\right\}}{E(\delta \mid Z)}\right]\right) \\
& \left(E\left\{\delta \dot{r}_{\vartheta}(X) \dot{r}_{\vartheta}(X)^{\top}\right\}-E\left[\frac{E\left\{\delta \dot{r}_{\vartheta}(X) \mid Z\right\} E\left\{\delta \dot{r}_{\vartheta}(X) \mid Z\right\}^{\top}}{E(\delta \mid Z)}\right]\right)^{-1} \frac{E\left\{\delta \dot{r}_{\vartheta}(X) \mid Z\right\}}{E(\delta \mid Z)} .
\end{aligned}
$$


Then

$$
\begin{aligned}
\phi_{*}(X, Z)= & t_{*}^{\top} \dot{r}_{\vartheta}(X)+g_{*}(Z) \\
= & \left\{\frac{1}{\mathbb{I}}\left(E\left\{\delta \dot{r}_{\vartheta}(X) \dot{r}_{\vartheta}(X)^{\top}\right\}-E\left[\frac{E\left\{\delta \dot{r}_{\vartheta}(X) \mid Z\right\} E\left\{\delta \dot{r}_{\vartheta}(X) \mid Z\right\}^{\top}}{E(\delta \mid Z)}\right]\right)^{-1}\right. \\
& \left.\left(E\left\{\dot{r}_{\vartheta}(X)\right\}-E\left[\frac{E\left\{\delta \dot{r}_{\vartheta}(X) \mid Z\right\}}{E(\delta \mid Z)}\right]\right)\right\}^{\top} \dot{r}_{\vartheta}(X) \\
& +\frac{\sigma^{2}}{E(\delta)}-\frac{1}{E(\delta) \mathbb{I}}+\frac{1}{E(\delta \mid Z) \mathbb{I}} \\
& -\frac{1}{\mathbb{I}}\left(E\left\{\dot{r}_{\vartheta}(X)^{\top}\right\}-E\left[\frac{E\left\{\delta \dot{r}_{\vartheta}(X) \mid Z\right\}}{E(\delta \mid Z)}\right]\right) \\
& \left(E\left\{\delta \dot{r}_{\vartheta}(X) \dot{r}_{\vartheta}(X)^{\top}\right\}-\right. \\
& \left.E\left[\frac{E\left\{\delta \dot{r}_{\vartheta}(X) \mid Z\right\} E\left\{\delta \dot{r}_{\vartheta}(X) \mid Z\right\}^{\top}}{E(\delta \mid Z)}\right]\right)^{-1} \frac{E\left\{\delta \dot{r}_{\vartheta}(X) \mid Z\right\}}{E(\delta \mid Z)} \\
= & \frac{\sigma^{2}}{E(\delta)}-\frac{1}{E(\delta) \mathbb{I}}+\frac{1}{E(\delta \mid Z) \mathbb{I}}-\frac{1}{\mathbb{I}}\left(E\left\{\dot{r}_{\vartheta}(X)^{\top}\right\}-E\left[\frac{E\left\{\delta \dot{r}_{\vartheta}(X) \mid Z\right\}}{E(\delta \mid Z)}\right]\right) \\
& \left(E\left\{\delta \dot{r}_{\vartheta}(X) \dot{r}_{\vartheta}(X)^{\top}\right\}-E\left[\frac{E\left\{\delta \dot{r}_{\vartheta}(X) \mid Z\right\} E\left\{\delta \dot{r}_{\vartheta}(X) \mid Z\right\}^{\top}}{E(\delta \mid Z)}\right]\right)^{-1} \\
& {\left[\dot{r}_{\vartheta}(X)-\frac{E\left\{\delta \dot{r}_{\vartheta}(X) \mid Z\right\}}{E(\delta \mid Z)}\right] . }
\end{aligned}
$$

Then using Corollary VI.14

$$
\begin{aligned}
\xi_{*}(X, Z, \epsilon)= & \frac{\epsilon}{E(\delta)}-\frac{l(\epsilon)}{E(\delta) \mathbb{I}}+\frac{l(\epsilon)}{E(\delta \mid Z) \mathbb{I}}-\frac{l(\epsilon)}{\mathbb{I}}\left(E\left\{\dot{r}_{\vartheta}(X)^{\top}\right\}-E\left[\frac{E\left\{\delta \dot{r}_{\vartheta}(X) \mid Z\right\}}{E(\delta \mid Z)}\right]\right) \\
& \left(E\left\{\delta \dot{r}_{\vartheta}(X) \dot{r}_{\vartheta}(X)^{\top}\right\}-E\left[\frac{E\left\{\delta \dot{r}_{\vartheta}(X) \mid Z\right\} E\left\{\delta \dot{r}_{\vartheta}(X) \mid Z\right\}^{\top}}{E(\delta \mid Z)}\right]\right)^{-1} \\
& {\left[\dot{r}_{\vartheta}(X)-\frac{E\left\{\delta \dot{r}_{\vartheta}(X) \mid Z\right\}}{E(\delta \mid Z)}\right] . }
\end{aligned}
$$

The final form is now straightforward by Lemma VI.11. 
Theorem VI.21 For the model

$$
Y=\vartheta^{\top} r_{\vartheta}(X)+\gamma(Z)+\epsilon
$$

where $Y$ is the response, $r_{\vartheta}(X)$ is the known parametric function and $\vartheta$ is the unknown parameter for the random covariate $X$ with dimension $k_{1}, \gamma(Z)$ is the unknown non-parametric component for the random covariate $Z$ with dimension $k_{2}$, and $\epsilon$ is the random error term with mean 0 and variance $\sigma^{2}$. Further assume $(X, Z)$ is independent of $\epsilon$. The responses are MAR with respect to the variable $\delta$ such that the observed data is $(X, Z, \delta, \delta Y)$. The canonical gradient for estimating $E(Y)$ is

$$
\begin{aligned}
g r_{\left(\vartheta_{*}, \gamma_{*}\right)}= & r_{\vartheta}(X)+\gamma(Z)-E\left\{r_{\vartheta}(X)+\gamma(Z)\right\}+\frac{\delta \epsilon}{E(\delta)}-\frac{\delta l(\epsilon)}{E(\delta) \mathbb{I}}+\frac{\delta l(\epsilon)}{E(\delta \mid Z) \mathbb{I}} \\
& -\frac{\delta l(\epsilon)}{\mathbb{I}}\left(E\left\{\dot{r}_{\vartheta}(X)^{\top}\right\}-E\left[\frac{E\left\{\delta \dot{r}_{\vartheta}(X) \mid Z\right\}}{E(\delta \mid Z)}\right]\right) \\
& \left(E\left\{\delta \dot{r}_{\vartheta}(X) \dot{r}_{\vartheta}(X)^{\top}\right\}-E\left[\frac{E\left\{\delta \dot{r}_{\vartheta}(X) \mid Z\right\} E\left\{\delta \dot{r}_{\vartheta}(X) \mid Z\right\}^{\top}}{E(\delta \mid Z)}\right]\right)^{-1} \\
& {\left[\dot{r}_{\vartheta}(X)-\frac{E\left\{\delta \dot{r}_{\vartheta}(X) \mid Z\right\}}{E(\delta \mid Z)}\right] . }
\end{aligned}
$$

Proof: The proof follows directly from Lemma VI.4 and Lemma VI.20

This canonical gradient can be used to check for whether an estimator is asymptotically efficient. If the influence function of the estimator matches this canonical gradient it is efficient. Unfortunately for this example an estimator has yet to be found which achieves this level of efficiency. The complexity can be observed by comparing this formula with Lemma II.2 where the model was a simple linear model with normally distributed errors. 


\section{VITA}

Scott Crawford was born in Cedar City, Utah. He received his B.S. in mathematics from Southern Utah University and M.S. in statistics from Brigham Young University in May 2004 and May 2006, respectively. He received his Ph.D. in statistics from Texas A\&M University in August 2012. He has accepted a position at the University of Wyoming as a lecturer to begin in August 2012. His current research interests lie in efficiency, missing data models, actuarial science, asymptotic theory, and mass spectrometry.

His permanent address is:

Department of Statistics, Texas A\&M University, 3143 TAMU, College Station, TX, 77843-3143. Email: crawford@stat.tamu.edu

The typist for this thesis was Scott Crawford. 